\title{
London Dispersion Helps Refine Steric A-Values: Dispersion Energy Donor Scales
}

\author{
Ephrath Solel, Marcel Ruth, and Peter R. Schreiner*
}

Institute of Organic Chemistry, Justus Liebig University, Heinrich-Buff-Ring 17, 35392 Giessen, Germany

*E-mail address: prs@uni-giessen.de

\section{Supporting Information}

\section{Table of Contents}

1. General methods S2

2. Benchmark Studies $\mathrm{S} 2$

3. Raw energy data used in benchmark studies S9

4. Dispersion Stabilization and Steric Part S18

5. Experimental A-values S20

6. Systems 2, 3, 4 S22

6. Cartesian Coordinates S25

7. Bibliography $\mathrm{S} 78$ 


\section{General Methods}

Geometries were optimized either at the MP2/aug-cc-pVTZ level of theory ${ }^{1}$ (using the aug-cc-pVTZ-PP² basis set with a relativistic ECP used for I) or at the B3LYP $3,4 /$ def2-TZVPP 5 level of theory, with and without Grimme's D3BJ dispersion correction. ${ }^{6,7}$ These computations were performed with Gaussian 16 revision C. $01^{8}$ with the int=ultrafine grid. Symmetry constraints were used where relevant. All minima were characterized with analytical Hessian calculations to ensure minima (i.e., no imaginary frequencies). Further single point computations on the optimized structures were done at the DLPNO-CCSD(T) ${ }^{9} /$ aug-cc-pVQZ (aug-cc-pVQZ-PP for iodine) level of theory using ORCA Version 4.2.1. ${ }^{10}$ DLPNO-CCSD(T) /aug-cc-pVQZ computations were performed with the frozen core approximation, with the RIJK approximation, using the aug-cc-pvqz/c aug-cc-pv5z/jk auxiliary basis sets, with verytightsSCF, TightPNO, and the "semi-canonical" perturbative triples correction approximation(T0). D4 corrections were computed with DFT-D4 version 2.5.0. ${ }^{11,12}$ NCIPlots $^{13}$ were generated with Multiwfn 3.7, ${ }^{14}$ and visualized using VMD. ${ }^{15} \mathrm{NCIPlots}$ were generated from the electron densities at the B3LYP/def2-QZVPP level of theory.

\section{Benchmark Studies}

In order to choose appropriate computational methods for structure optimization, energy computations, and dispersion evaluation, we performed benchmark studies. We chose the MP2 $a b$ initio method for geometry optimizations, as it is suitable for getting accurate geometries of organic molecules. We compared the optimized geometries at this method with several basis sets against the experimental structures of cyclohexane. There are three experimental structures- s1, s2, s3, all of them derived from the same experimental microwave rotational constants but differing in the method used for fitting and structure determination. ${ }^{16-18}$ From our results (Figure S1, Table S1), we can see that the MP2/aug-cc-pVTZ geometries show deviations of less than $0.01 \AA$ for bond lengths and $0.3^{\circ}$ for angles, relative to the s2 experimental results. From comparison to all three series of experimental bond lengths it is clear that the smallest MAEs are achieved with the aug-cc-pVTZ basis set. Quadruple zeta (QZ) basis sets did not perform better than the more efficient triple zeta (TZ) basis set. These results are in accordance with previous studies that showed that geometry optimizations at MP2 triple-zeta quality basis sets give very similar results to quadruple-zeta basis sets, ${ }^{19}$ sometimes even showing smaller errors with TZ as compared to QZ. ${ }^{20}$ As a result, the MP2 level of theory with the aug-cc-pVTZ basis set was chosen for future structure optimizations.

A

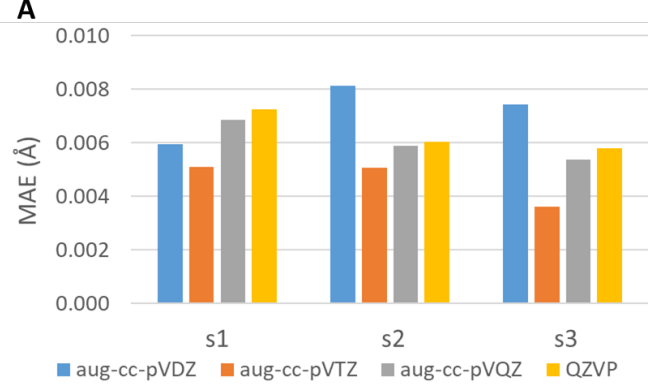

B

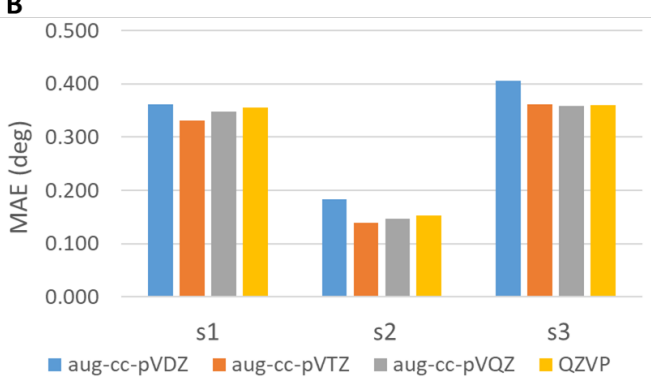

Figure S1. MAE (mean absolute error) for bond lengths and angles for structures optimized at the MP2 level of theory with various basis sets. 
Table S1: bond lengths (in $\AA$ ) and angles of cyclohexane from geometry optimizations at the MP2 level of theory with basis sets aug-cc-pVDZ/aug-cc-pVTZ/aug-cc-pVQZ/QZVP, the experimental parameters (with experimental errors in parenthesis), and the difference between computational and experimental results at the different levels.

\begin{tabular}{|c|c|c|c|c|c|c|c|c|c|c|}
\hline & C-C & C- $\mathrm{H}_{\mathrm{ax}}$ & C- $\mathrm{H}_{\mathrm{eq}}$ & C-C-C & $\mathrm{H}-\mathrm{C}-\mathrm{H}$ & $\mathrm{CCH}_{\mathrm{ax}}$ & $\mathrm{CCH}_{\mathrm{eq}}$ & CCCC & $\mathrm{MAE}_{\mathrm{B}}$ & $\mathrm{MAE}_{\mathrm{A}}$ \\
\hline aug-cc-pVDZ & 1.537 & 1.106 & 1.104 & 110.942 & 106.903 & 109.110 & 110.337 & 56.205 & & \\
\hline aug-cc-pVTZ & 1.527 & 1.095 & 1.092 & 110.977 & 106.903 & 109.056 & 110.372 & 56.110 & & \\
\hline aug-cc-pVQZ & 1.524 & 1.094 & 1.090 & 110.966 & 106.877 & 109.050 & 110.395 & 56.138 & & \\
\hline QZVP & 1.524 & 1.093 & 1.090 & 110.963 & 106.862 & 109.058 & 110.396 & 56.146 & & \\
\hline \multicolumn{11}{|c|}{ Experimental } \\
\hline s1 & $\begin{array}{c}1.5300 \\
(32)\end{array}$ & $\begin{array}{c}1.1013 \\
(39)\end{array}$ & $\begin{array}{c}1.0933 \\
(15)\end{array}$ & $\begin{array}{c}111.28 \\
(30)\end{array}$ & $\begin{array}{c}106.65 \\
(27)\end{array}$ & $\begin{array}{c}108.83 \\
(92)\end{array}$ & $\begin{array}{c}110.55(2 \\
9)\end{array}$ & $\begin{array}{c}55.26 \\
(82)\end{array}$ & & \\
\hline s2 & $\begin{array}{c}1.5359 \\
\text { (3) }\end{array}$ & $\begin{array}{c}1.0979 \\
(21)\end{array}$ & $\begin{array}{c}1.0887 \\
(57)\end{array}$ & $\begin{array}{c}111.08 \\
(5)\end{array}$ & $\begin{array}{c}106.99 \\
(92)\end{array}$ & $\begin{array}{c}108.90 \\
(6)\end{array}$ & $\begin{array}{c}110.43(4 \\
4)\end{array}$ & $\begin{array}{c}55.82 \\
(13)\end{array}$ & & \\
\hline s3 & $1.536(3)$ & $1.098(1)$ & $1.095(1)$ & $111.1(2)$ & $107.6(2)$ & $108.8(2)$ & $110.2(2)$ & $\begin{array}{l}55.7 \\
(2)\end{array}$ & & \\
\hline \multicolumn{11}{|c|}{ Difference relative to $s 3$} \\
\hline aug-cc-pVDZ & 0.0009 & 0.0085 & 0.0086 & -0.1580 & -0.6970 & 0.3100 & 0.1370 & 0.5050 & 0.006 & 0.361 \\
\hline aug-cc-pVTZ & -0.0091 & -0.0031 & -0.0031 & -0.1230 & -0.6970 & 0.2560 & 0.1720 & 0.4100 & 0.005 & 0.332 \\
\hline aug-cc-pVQZ & -0.0116 & -0.0045 & -0.0045 & -0.1340 & -0.7230 & 0.2500 & 0.1950 & 0.4380 & 0.007 & 0.348 \\
\hline QZVP & -0.0120 & -0.0049 & -0.0049 & -0.1370 & -0.7380 & 0.2580 & 0.1960 & 0.4460 & 0.007 & 0.355 \\
\hline \multicolumn{11}{|c|}{ Difference relative to s2 } \\
\hline aug-cc-pVDZ & 0.0010 & 0.0086 & 0.0149 & -0.14 & -0.09 & 0.21 & -0.09 & 0.38 & 0.008 & 0.183 \\
\hline aug-cc-pVTZ & -0.0091 & -0.0030 & 0.0032 & -0.10 & -0.09 & 0.16 & -0.06 & 0.29 & 0.005 & 0.139 \\
\hline aug-cc-pVQZ & -0.0115 & -0.0044 & 0.0018 & -0.11 & -0.11 & 0.15 & -0.04 & 0.32 & 0.006 & 0.146 \\
\hline QZVP & -0.0119 & -0.0048 & 0.0014 & -0.12 & -0.13 & 0.16 & -0.03 & 0.33 & 0.006 & 0.153 \\
\hline \multicolumn{11}{|c|}{ Difference relative to $s 1$} \\
\hline aug-cc-pVDZ & 0.0069 & 0.0052 & 0.0103 & -0.34 & 0.25 & 0.28 & -0.21 & 0.94 & 0.007 & 0.406 \\
\hline aug-cc-pVTZ & -0.0031 & -0.0063 & -0.0014 & -0.30 & 0.25 & 0.23 & -0.18 & 0.85 & 0.004 & 0.362 \\
\hline aug-cc-pVQZ & -0.0056 & -0.0078 & -0.0028 & -0.31 & 0.23 & 0.22 & -0.16 & 0.88 & 0.005 & 0.359 \\
\hline QZVP & -0.0060 & -0.0081 & -0.0032 & -0.32 & 0.21 & 0.23 & -0.15 & 0.89 & 0.006 & 0.359 \\
\hline
\end{tabular}

Secondly, we wanted to identify a DFT functional for computing energies and LD interactions, as MP2 is not so reliable for energies. Single point energy computations of various monosubstituted cyclohexanes (optimized at the MP2/aug-cc-pVTZ level of theory) with several DFT functionals (using the def2-TZVPP basis set) were compared to DLPNO-CCSD(T)/aug-cc-pVQZ energies (with the aug-cc-pVQZ-PP basis set for iodine). All DFT functionals gave errors smaller than the usual requirement of $1 \mathrm{kcal} \mathrm{mol}^{-1}$ (Figure S2), but considering that the A-value can exhibit values smaller than $1 \mathrm{kcal} \mathrm{mol}^{-1}$, we were striving to achieve higher accuracy in our computations. Most of the DFT functionals gave much smaller errors when a dispersion correction was used. 


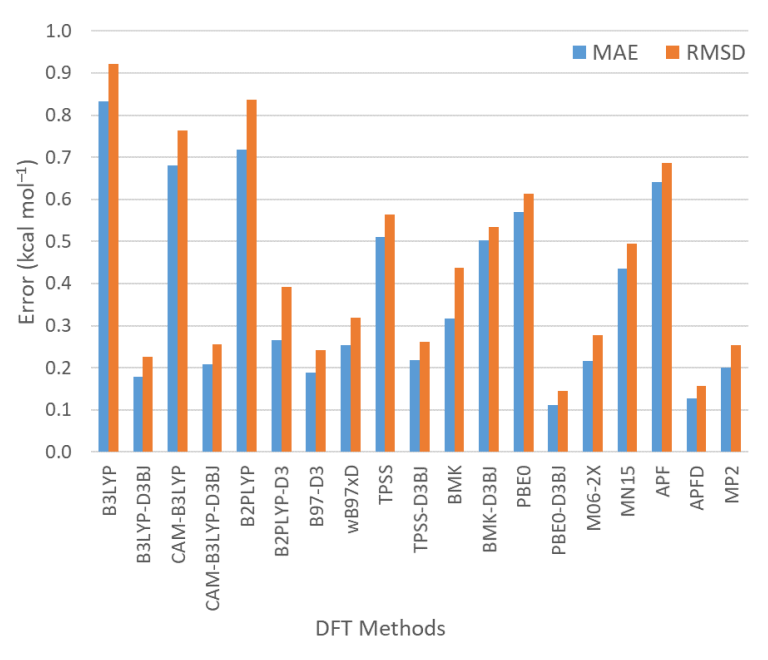

Figure S2. MAE and RMSD of the DFT single point energies, relative to single point energy computations at DLPNO-CCSD(T)/aug-cc-pVQZ. The basis set used with all DFT functionals is def2-TZVPP.

Some of the best-performing functionals were chosen for the next step. In this step, we checked the quantification of dispersion interactions from the DFT dispersion corrections. In the monosubstituted cyclohexanes we cannot quantify the dispersion part by using energy decomposition analysis methods like LED-DLPNO ${ }^{9}$ or SAPT, ${ }^{21,22}$ because we are looking at intramolecular dispersion interactions, and these methods are more reliable for intermolecular interactions. Note, that LED-DLPNO can be used also for intramolecular interactions, as demonstrated in some previous studies. ${ }^{23-26}$ However, treatment of fragments bound via a covalent bond can cause problematic results, as we show below (see method validation part). We decided to use the size of Grimme's dispersion correction as a measure of dispersion energy. For this, we used DFT functionals with and without a dispersion correction to evaluate the dispersion interaction in a set of noncovalent dimers formed between cyclohexane and either fluorine, chlorine, bromine, iodine or methane (all dimers were optimized in $C_{3 v}$ symmetry). However, DFT functionals include some dispersion in their exchange-correlation part. The D3BJ correction, by design, does not include only dispersion terms but also corrections for systematic errors in the DFT functionals. For this reason the dispersion corrections have different values for different functionals, and they usually underestimate the dispersion. To account for this, we tested also Truhlar's damped dispersion based on D3 and SAPT (DD3S). The design of this method is based on Grimme's dispersion correction equations, with parameters that were adjusted from a comparison to SAPT dispersion results (D3BJ parameters: $s_{6}=1.0, s_{8}=2.0, a_{1}=0.5299, a_{2}=2.2$ ). ${ }^{27}$ In order to isolate only the dispersion interaction between the two monomers (and not within the monomers), the D3BJ-dispersion energy of each monomer (at the dimer's geometry) was subtracted from the total dispersion energy of the dimer. From comparison of our results to the dispersion part computed from DLPNO-LED (Figure S3) we can conclude that B3LYP with the D3BJ correction is the best performing DFT functional for our system, giving results closest to the DLPNO-LED ones. The B3LYP functional, being a relatively repulsive functional that does not take into account LD effects intrinsically, was shown also in previous studies to give LD corrections in a good agreement with LED results. ${ }^{28}$ The DD3S dispersion gave higher values, overestimating dispersion energy with respect to DLPNO-LED in these systems. 


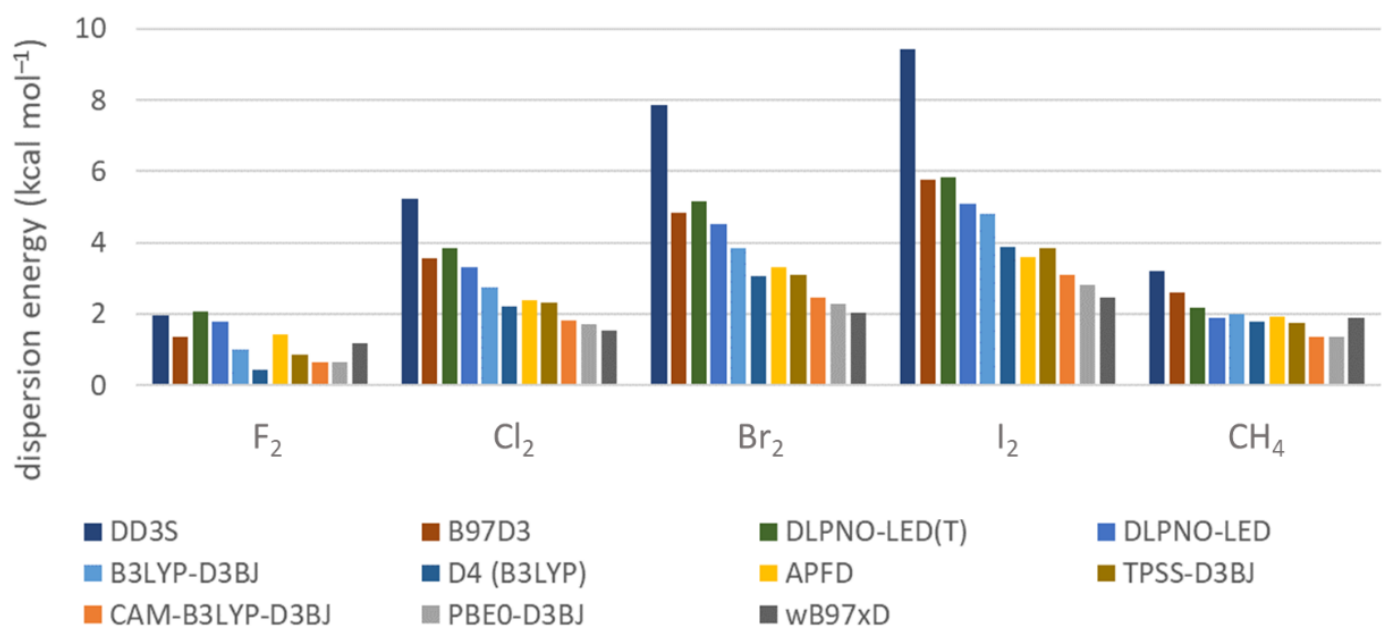

Figure S3. Dispersion energy for a set of molecules interacting with cyclohexane, computed from DLPNO-LED analysis and various DFT/def2-TZVPP functionals. DLPNO-LED refers to the sum of strong and weak pair interactions, while DLPNO-LED(T) includes also an effective triples correction, as in reference ${ }^{29}$.

\section{Method Validation - LD}

In addition to our previous benchmark studies, here we compare the dispersion stabilization from our computations (D3BJ corr., see details in the main text) to the state-of-the-art methods for computing dispersion directly from excited pair orbital interactions, namely LED from DLPNO-CCSD(T). It should be remembered, as noted above, the LED analysis is good for intermolecular interactions, but could be problematic for intramolecular cases. We designed three measures of dispersion energy (see also Fig. S4):

1. Measure A: Intramolecular LED. Here the treatment of the bond between the two groups can affect the results. Table S2 shows that the assignment of the bonding orbital between the fragments is not consistent for all $\mathrm{R}$ groups. For groups that are more electron-withdrawing (halogens, $\mathrm{CCH}, \mathrm{CN}, \mathrm{Ph}$ ) it is assigned to the $\mathrm{R}$ group, but this is the case also for $\mathrm{CCl}_{3}$, unlike the other $\mathrm{CX}_{3}$ groups. Here (and also in measure $\mathrm{B}$ ) we add the effective triples correction ${ }^{29}$ to the dispersion energy.

2. Measure B: Intermolecular LED, computed from the geometries of the original monosubstituted cyclohexane, with the carbon bonded to $\mathrm{R}$ removed (Fig. S4). Instead of the carbon, three hydrogens are added at fixed bond lengths $\left(\mathrm{C}_{\mathrm{sp} 3}-\mathrm{H} 1.09 \AA, \mathrm{C}_{\mathrm{sp}}-\mathrm{H} 1.08 \AA . \mathrm{C}_{\mathrm{Ar}}-\mathrm{H}\right.$

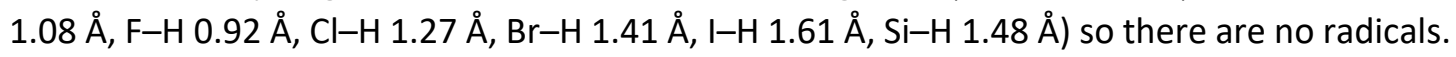
In this case any $L D$ interactions between the $R$ group and the hydrogen that was removed (on carbon number 1 in Fig. S4) are not taken into account, which is expected to reduce the LD part especially of large $\mathrm{R}$ groups that are pushed away from the axial hydrogens and closer to the $\mathrm{CH}$, in the axial conformer. Also, the generally longer $\mathrm{C}-\mathrm{R}$ bonds in the axial conformer would be expected to make LD interactions between the three new added hydrogens stronger in the equatorial conformer.

3. Measure C: A method to compute the dispersion in analogy to the method we use to compute the D3BJ corrected dispersion. This was done by using the difference between the DLPNO$\operatorname{CCSD}(T)$ and $\mathrm{HF}$ energies as an estimate of the dynamic electron correlation energy. The intrafragment correlation energy of both the $\mathrm{Cy}-\mathrm{H}$ and $\mathrm{R}-\mathrm{H}$ groups was subtracted from the total correlation energy of the monosubstituted cyclohexane. In the resulting interfragment 
correlation energy, not just LD is taken into account, but also other dynamic correlation interactions, such as charge transfer between the groups.

4. Measure D: A method to compute the dispersion in analogy to the method we use to compute the D3BJ corrected dispersion, and measure C. Here we use the modified dispersion parameters of the DD3S method. ${ }^{27}$
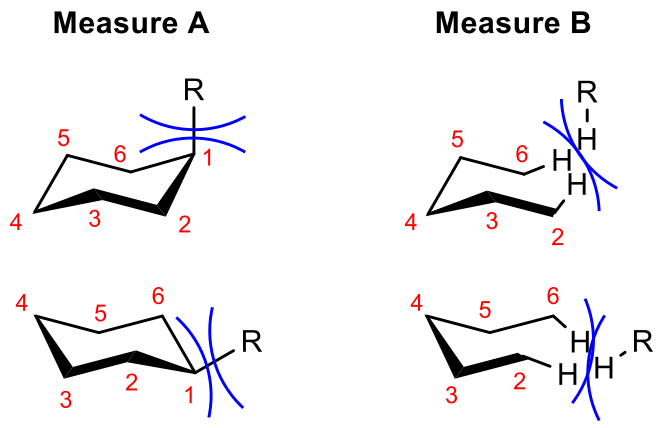

Figure S4. The different measures for computing the dispersion energy.

Table S2. Populations of internal orbitals onto fragments, from DLPNO-LED analysis. The population of the $\mathrm{C}_{\mathrm{Cy}-\mathrm{R}}$ bonding orbital as divided between the two fragments $(1-\mathrm{Cy}, 2-\mathrm{R})$, and the fragment this orbital was assigned to, in measure $A$.

\begin{tabular}{lcccccc}
\hline & \multicolumn{3}{c}{ Axial conformer } & \multicolumn{3}{c}{ Equatorial conformer } \\
$\mathbf{R}$ & Pop. 1 & Pop. 2 & assigned to frag. & Pop. 1 & Pop. 2 & assigned to frag. \\
\hline $\mathbf{F}$ & 0.293 & 0.756 & 2 & 0.271 & 0.761 & 2 \\
$\mathbf{C l}$ & 0.519 & 0.623 & 2 & 0.598 & 0.622 & 2 \\
$\mathbf{B r}$ & 0.441 & 0.648 & 2 & 0.400 & 0.680 & 2 \\
$\mathbf{l}$ & 0.583 & 0.608 & 2 & 0.494 & 0.685 & 2 \\
$\mathbf{C C H}$ & 0.408 & 0.696 & 2 & 0.372 & 0.820 & 2 \\
$\mathbf{C N}$ & 0.471 & 0.724 & 2 & 0.466 & 0.819 & 2 \\
$\mathbf{M e}$ & 0.636 & 0.482 & 1 & 0.754 & 0.408 & 1 \\
$\mathbf{E t}$ & 0.622 & 0.471 & 1 & 0.622 & 0.503 & 1 \\
$\mathbf{i P r}$ & 0.598 & 0.463 & 1 & 0.491 & 0.536 & 2 \\
$\mathbf{t B u}$ & 0.638 & 0.436 & 1 & 0.52 & 0.578 & 2 \\
$\mathbf{P h}$ & 0.515 & 0.73 & 2 & 0.493 & 0.735 & 2 \\
$\mathrm{CF}_{3}$ & 0.624 & 0.543 & 1 & 0.681 & 0.561 & 1 \\
$\mathrm{CCl}_{3}$ & 0.536 & 0.593 & 2 & 0.554 & 0.631 & 2 \\
$\mathrm{CBr}_{3}$ & 0.576 & 0.485 & 1 & 0.624 & 0.498 & 1 \\
$\mathrm{Cl}_{3}$ & 0.582 & 0.481 & 1 & 0.666 & 0.473 & 1 \\
$\mathrm{SiMe}_{3}{ }^{a}$ & 0.951 & 0.190 & 1 & 0.913 & 0.123 & 1 \\
\hline
\end{tabular}

The various estimations for the LD energy are plotted in Fig. S5. 
A

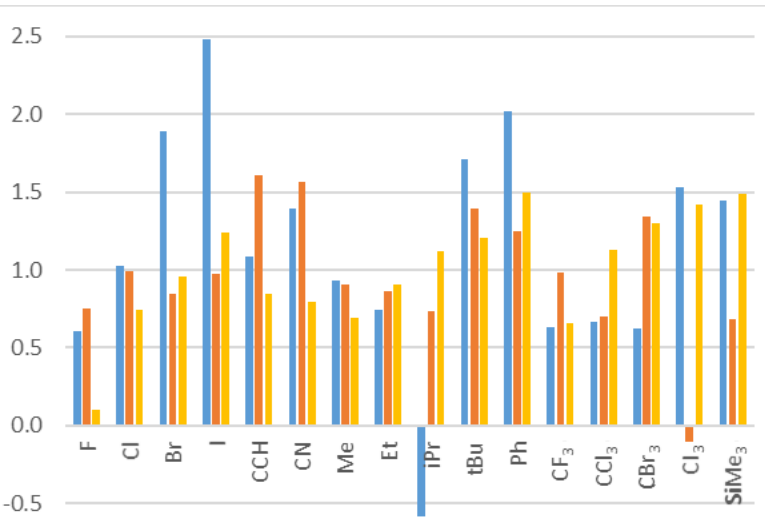

$-1.0$

Measure A Measure B D3BJ corr.
B

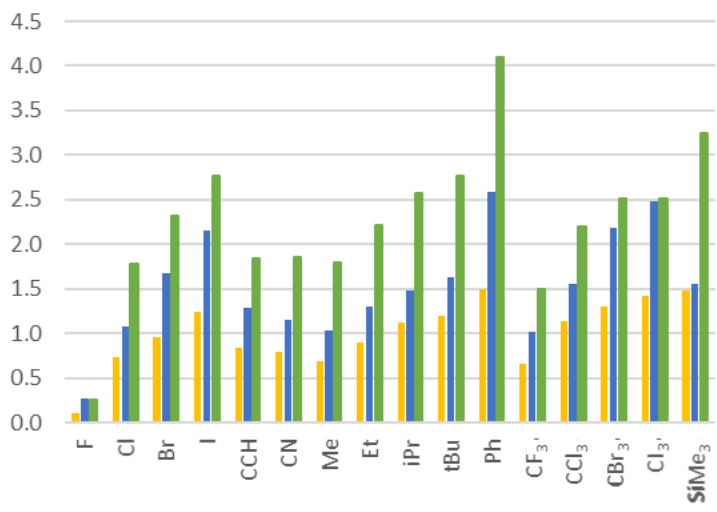

D3BJ corr. Measure C DD3S

Figure S5. Comparison of the different measures of the dispersion part.

Fig. S5 shows that there are two outliers: for $i \mathrm{Pr}$ in measure $\mathrm{A}$ and $\mathrm{Cl}_{3}$ in measure $\mathrm{C}$, the results are negative, unlike the results from all other measures. For $i \operatorname{Pr}$ (and also $t \mathrm{Bu}$ ) the bonding $\mathrm{C}-\mathrm{R}$ is populated roughly to the same extent on both fragments (Table S2), but in the two conformers it is assigned to different fragments, and this apparently affects the results.

In general, the energies from measure $C$ are larger than the D3BJ corrected LD energy. This could result from charge transfer energies which are included in measure $C$, or from an underestimation of LD by the D3BJ correction. As LD interactions arise fully from electron correlation, but not all electron correlation effects are dispersion effects, measure $\mathrm{C}$ can be considered as an upper limit for the strength of LD interactions. Despite the differences in numerical values, measure C and the D3BJ corrected LD energies qualitatively agree very well.

In general, the DD3S correction gives values higher than the DLPNO correlation, overshooting the dispersion in these molecules.

The D3BJ corrected $L D$ energies are generally closer to one of measures $A$ and $B$ than to measure $C$. For $\mathrm{Me}$ and $\mathrm{Et}$, for example, there is very good agreement between the D3BJ energies and measures $A$ and $B$. Even though the inconsistencies between the results of measures $A, B$, and $C$ and the inherent problems with all these methods makes quantitative comparisons difficult, these are not required as our aim is to establish relative dispersion energy donor abilities and make general remarks about steric measures. Hence, the by-and-large good qualitative agreement of the key measures and the D3BJ correction also allow qualitative rankings of groups to be made. Moreover, it turns out that D3BJ does not seem to overshoot in estimating the "true" dispersion energy corrections. This supports our qualitative conclusions that LD interactions are significant, relative to steric hindrance, also in small organic systems such as substituted cyclohexanes.

In analogy to our DED scales, we took the results of measure $C$ as a scale of interfragment electron correlation. Subtracting these values from the computed total energy differences between the axial and equatorial conformers at DLPNO-CCSD(T), we get also a scale of the steric part, the part that does not include any interfragment electron correlation (Fig. S6). These scales, although their values differ from the dispersion-based scales in the main manuscript, also demonstrate our key conclusions. They show that electron correlation (a large part of which is LD) is significant for this system, and is on the 
same order of magnitude as the steric hindrance. Also, for small groups (halogens, $\mathrm{CN}, \mathrm{CCH}$ ) most of the steric hindrance is compensated by interfragment electron correlation, significantly lowering the total energy between the conformers. The order of the groups as electron-correlation donors also matches very closely our computed DED order. The main differences are that measure $\mathrm{C}$ places $\mathrm{Br}$ as a better electron correlation donor than all alkyl groups, and places TMS lower than $t \mathrm{Bu}, \mathrm{I}, \mathrm{CBr}_{3}, \mathrm{Cl}_{3}$.

A

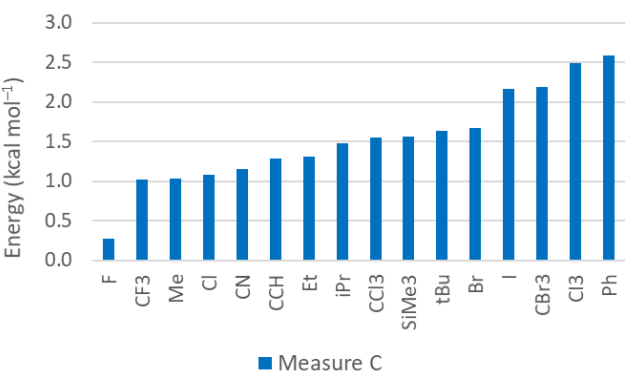

B

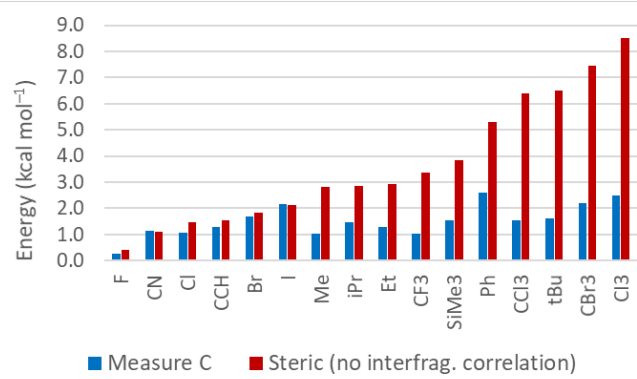

C

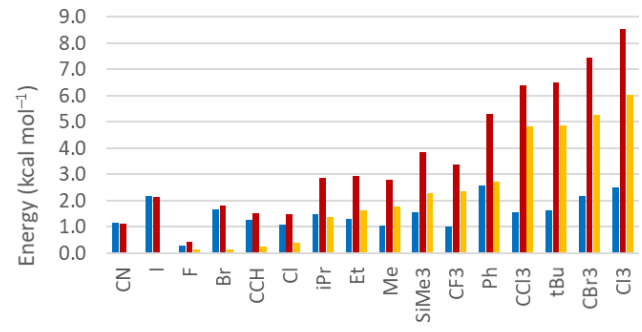

- Measure C

- Steric (no interfrag. correlation)

Total Energy Difference

Figure S6. The corrected interfragment electron correlation energy from measure $C$, the purely steric part left after addition of the correlation energy stabilization to the total energy, and the total energy (DLPNO-CCSD(T)), for monosubstituted cyclohexanes. Groups are arranged from lowest to highest according to the LD stabilization $(A)$, the steric part (B), or the total energy (C). 


\section{Raw energy data used in benchmark studies}

Table S3. Single point energies (in a.u.) computed with various DFT functionals, all with the def2-TZVPP basis set. From MP2/aug-cc-pVTZ optimized geometries

\begin{tabular}{|c|c|c|c|c|c|c|c|c|c|c|c|c|c|c|c|c|c|c|c|}
\hline olecule & LYP & YP-D3BJ & CAM-B3LYP & CAM-B3 & 2PLYP & PLYP-D3 & 7-D3 & $397 \times D$ & IPSS & PSS-D3BJ & BMK & BMK-DЗBJ & PBEO & \begin{tabular}{|l|} 
PBEO-DZBJ \\
\end{tabular} & M06-2x & MN15 & APF & APFD & MP2 \\
\hline Feq & 5928 & 5.28325 & 94 & 51 & 34.96920 & 4.98115 & 5095 & 699 & 35.29426 & 13 & 35.03511 & 129 & .86644 & 05 & 089 & .85823 & 4.97533 & 987397 & -334.4898 \\
\hline Fax & .2561316 & 5.28289 & 5.0897 & 35.1 & 34.96890 & 34.98090 & 5.05022 & 35.15660 & 35.29381 & 35.31376 & 35.03494 & 35.05122 & 34.86590 & |-334.87957| & -335.1 & 334.85829 & 4.97476 & -334. & 34. \\
\hline $\mathrm{Cleq}_{\mathrm{eq}}$ & 5.6007714 & 5.63132 & 456 & 95.468 & 5.22302 & 5.23691 & 15.46027 & 95.51395 & 95.63981 & 6252 & 35.27832 & 95.29775 & 85.14200 & $|-695.15761|$ & -695.45221 & 595.17208 & 95.27342 & -695.2856016 & |-694.4 \\
\hline $\mathrm{Clax}_{\mathrm{ax}}$ & 2039 & 5.63084 & & $95.4 \mathrm{rat}$ & 5.22191 & 5.23635 & 5.45967 & 95.51304 & 5.63872 & 221 & 5.27766 & 5.29804 & 95.14087 & \begin{tabular}{|l|}
$\mid-695.15702$ \\
\end{tabular} & -695.45196 & 995.17231 & 95.27220 & \begin{tabular}{|l|l|} 
& -695.2 \\
\end{tabular} & -694 \\
\hline $1 \mathrm{Br}_{\mathrm{eq}}$ & -2809.55558 & -2809.5873 & -2809.5072 & 809 & 08.91200 & -2808.92659 & -2810.69350 & -2809.51847 & -2809.46886 & -2809.49236 & -2808.04480 & -2808.06573 & -2808. & -28 & -2809.44669 & 809.54163 & 2809.14956 & -2805 & \begin{tabular}{|l}
-2807 \\
\end{tabular} \\
\hline $\mathrm{Br}_{\mathrm{ax}}$ & .553605 & 09.5868 & 年 & -2809.520844 & .91063 & 597 & 80 & .51739 & 63 & 14 & 4378 & 6610 & -28 & -28 & 39 & 54191 & 481 & -2809.162777 & -2 \\
\hline leq & 535 & 3.20579 & .93339 & -532.9 & 27 & 2.69386 & 3.44246 & 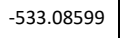 & 33.00073 & 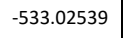 & $11.88 / 29$ & 31.91044 & 32.79776 & -532.81480 & (5) & 32.04081 & 532.93428 & $\mid-532.9$ & 更 \\
\hline $1 \operatorname{lax}_{\text {ax }}$ & 3.1700771 & 3.20539 & 2.93199 & -532.948 & 2.67670 & 2.69330 & 3.44189 & 476 & 2.99941 & 33.02537 & 531.88582 & 531.91098 & 532.79643 & \begin{tabular}{|l|} 
\\
\end{tabular} 32.81438 & -532.88106 & 532.04110 & 532.93282 & -532.96 & 525.0 \\
\hline $\mathrm{CCH}_{\text {eq }}$ & 2.1411023 & 12.1735 & -311.9481 & 1.5 & 1.84013 & 1.85484 & 1.93002 & 12.03736 & 12.18420 & 12.20843 & 11.89871 & B11.91911 & -311.74626 & $|-311.76297|$ & -311. & -311.72365 & 311.8580 & $\mid-311.8$ & $\mid-311.3$ \\
\hline $\mathrm{CCH}_{\mathrm{ax}}$ & 2.1392639 & 2.17296 & 1.94661 & 11.96 & 1.83904 & 1.85440 & 1.92966 & 12.03686 & 12.18296 & 21 & 11.89802 & 311.91946 & -311.74508 & $\mid-311.76251$ & -311 & 311.72403 & 311.8567 & $\mid-311.8$ & $\mid-311$. \\
\hline $1 \mathrm{CN}_{\mathrm{eq}}$ & 8.2459428 & 8.27723 & 8.05572 & 28.06 & 27.94576 & 27.95994 & 28.02811 & 28.13848 & 28.29264 & 328.31604 & -328.00931 & -328.02889 & -327.84043 & \begin{tabular}{|l|}
$\mid-327.85654$ \\
\end{tabular} & -328.09276 & -327.81713 & 327.95406 & -327.9672929 & $\mid-327.43$ \\
\hline $1 \mathrm{CN}_{\mathrm{ax}}$ & 8.2446593 & 8.27717 & 8.05482 & -328.0696331 & 27.94517 & 27.95995 & 28.02820 & 28.13839 & 28.29184 & 328.31618 & 328.00910 & -328.02962 & $\mid-327.83976$ & $\mid-327.85654$ & -328.09311 & -327.81797 & -327.95327 & -327.96718 & |-327.4389538 \\
\hline $1 \mathrm{Me}_{\mathrm{eq}}$ & 006 & 5.33377 & 5.12653 & -275.14 & 75.02165 & 5.03611 & 5.11956 & 75.21803 & 75.33728 & 275.36139 & 275.08138 & -275.10104 & -274.95155 & \begin{tabular}{|l|}
-274.96813 \\
\end{tabular} & -275.1 & 274.92144 & 275.05358 & -275.0 & -274.5755 \\
\hline $1 \mathrm{Me}_{\mathrm{ax}}$ & 5.297872 & 5.33102 & 15173 & -2 & 55.01832 & 75.03327 & 75.11682 & 275.21550 & 275.33402 & 92 & 53 & 93 & -274.9 & -274 & & 12 & 007 & & -274 \\
\hline $1 \mathrm{Eteq}_{\mathrm{eq}}^{\mathrm{A}}$ & 5 & 39 & 14.42881 & -314.4 & .30904 & 14.32608 & .42155 & .53432 & 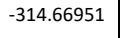 & 94 & & & 84 & 46 & & & & & \\
\hline 1 Eteq B & 1 & 6513 & 4.42708 & -314.4445464 & 14 & 14.32477 & 14.42034 & -314.53320 & -314.66791 & -314 & 96 & 64 & 314. & -314 & -314 & 105 & 357 & 506 & -313 \\
\hline $1 \mathrm{Etax}^{\mathrm{C}} \mathrm{C}$ & & $=$ & & & & & 4.41905 & -314.53201 & -314.66621 & & -314.37446 & & 38 & -31 & & & & & \\
\hline $\operatorname{dox}(103)$ & & - & & & & & & & & & & & & & & & & & \\
\hline $1 \mathrm{iPr}_{\mathrm{eq}} \mathrm{A}$ & 99 & 19 & -353.73061 & -353.7511362 & 3.08446 & 3.10470 & 69 & -353.85072 & 354.00093 & -354.03469 & 09 & 83 & 71 & $-35=$ & -353 & 53.46980 & 631 & 308 & -353 \\
\hline $1 \mathrm{iPr}_{\mathrm{eq}} \mathrm{B}$ & 1 & 39 & -353.73042 & -353.7511936 & -353.08432 & 6 & 53.72400 & -353.85107 & -354.00078 & -354.03487 & -353.67319 & 25 & 55 & 16 & -353.76856 & 18 & -353.63615 & 141 & -353.0263624 \\
\hline LiPrax $C$ & 3.9505647 & 53.997 & . & -353.7486139 & 40 & 10149 & 72161 & 32 & 年 & 288 & 46 & 52 & 237 & 676 & 19 & 55 & 283 & t171 & -3 \\
\hline $1+\mathrm{tBu}$ eq & 8 & 68 & -393.0321 & -393.0568461 & 99 & 16 & 65 & 94 & 84 & 14 & 44 & 80 & 235 & 34 & -393.07711 & 53 & 2696 & 4728 & 574 \\
\hline $1+B u_{a x}$ & 02 & 22 & -393.0232 & -393.0489846 & 45 & 45 & 21 & 84 & 35 & 03 & 32 & 02 & 356 & 258 & -393.06963 & 24 & 1803 & 483 & 457 \\
\hline $1 \mathrm{Ph}_{\mathrm{eq}} \mathrm{A}$ & -467.11 & 7.15841 & 5.82082 & 5.84 & 5.66200 & 5.68656 & 5.79271 & 6.95184 & 7.17521 & 7.21527 & 149 & 3621 & -466.52702 & $\mid-466.55484$ & 10 & 66.48904 & 66.69030 & 4742 & -465 \\
\hline $1 \mathrm{Ph}_{\mathrm{ax}} \mathrm{B}$ & -467.0980276 & \begin{tabular}{|l|} 
\\
\end{tabular} & \begin{tabular}{|l|} 
\\
\end{tabular} & 6.840 & 6.65624 & 6.68194 & 6.78814 & 6.94735 & 7.16944 & 7.21136 & 6.74631 & 6.78280 & -466.52127 & 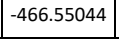 & 3011 & 60 & 66.68431 & 0827 & \begin{tabular}{|l|l|} 
& -465.9134446 \\
\end{tabular} \\
\hline $1 \mathrm{Ph}_{\mathrm{ax}} \mathrm{C}$ & -467.0976012 & -467.15269 & -466.81343 & 6.8394442 & 6.65505 & 6.68044 & 6.78701 & 6.94584 & 7.16823 & 7.20967 & 6.74465 & 6.78073 & -466.51976 & $|-466.54860|$ & 728 & -466.48191 & 66.68296 & 3586 & -465. \\
\hline 3eq & -573.1697964 & 3.20354 & 2.94814 & 2.96 & 2.12981 & 2.14503 & 2.84382 & 3.01032 & 73.22611 & 573.25138 & -572.85744 & -572.87830 & -572.55556 & $\mid-572.57295$ & -572.94401 & -572.58051 & 572. & 9598 & \\
\hline
\end{tabular}




\begin{tabular}{|c|c|c|c|c|c|c|c|c|c|c|c|c|c|c|c|c|c|c|c|}
\hline $\mathrm{CF}_{3 a x}$ & 3.1657076 & 3.20024 & 2.94423 & 72.9599424 & 2.12542 & 72.14106 & 72.83999 & 73.00677 & 73.22209 & -573.24802 & -572.85380 & -572.87534 & -572.55144 & -572.56931 & -572.94048 & -572.57678 & $\mid-572.71616$ & -572.7368238 & -572.0188969 \\
\hline $\mathrm{Cl}_{3 \mathrm{eq}}$ & & & & 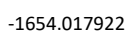 & & - & - & & -1 & 80 & 1 & & & & & & & & \\
\hline $\mathrm{Cl}_{3 \mathrm{ax}}$ & & 1. & & & & & 376 & & 330 & & 0 & 9 & & & & 660 & 3.55950 & & \\
\hline $\mathrm{CBr}_{3 \mathrm{zeq}}$ & 51 & 66.0695 & | 7996.1478 & -7996.173743 & 6652 & 89216 & 37 & 228 & 416 & 37 & 297 & 106 & 6. & 39 & 87 & 36 & 5.19095 & 198 & 249 \\
\hline $\mathrm{CBr}_{3 a x}$ & 915 & . 0616 & -7996.1381 & 996.165114 & 09 & -7 & 46 & . & -2 & 95 & 359 & 43 & - & 80 & 68 & 382 & 18132 & 04 & 007 \\
\hline $\mathrm{Cl}_{3 \text { eq }}$ & 6.86685 & 66.9276 & 66.4279 & -1 & & -1 & & & & & & & & 14 & 85 & & 166.54562 & & 508 \\
\hline $\mathrm{Cl}_{\mathrm{zax}}$ & -1166.855621 & 66.9182 & & & & & & & & & & & & & & & 1166.53439 & & 712 \\
\hline neseq & 09 & 1. & -64 & & & & & & & & & & 573 & 34 & 14.49145 & 44 & -644.29664 & 294 & 951 \\
\hline $\mathrm{Me}_{3 \mathrm{ax}}$ & 921 & .77294 & 4.47392 & 46 & 77 & 145 & 550 & 76 & & 56 & & & 197 & 194 & 332 & 366 & & 124 & 974 \\
\hline
\end{tabular}

Table S4. DLPNO-LED analysis ( energies in a.u.) of cyclohexane $\cdots \cdot \mathrm{X}$ dimers $\left(\mathrm{X}=\mathrm{F}_{2}, \mathrm{Cl}_{2}, \mathrm{Br}_{2}, \mathrm{I}_{2}, \mathrm{CH}_{4}\right.$ )

\begin{tabular}{|l|c|c|c|c|c|}
\hline & $E^{\text {tot }}$ & $E^{\text {disp }}(\mathbf{S P})$ & $E^{\text {disp }}(\mathbf{W P})$ & inter Triples & inter SP total \\
\hline $\mathrm{C} \boldsymbol{y} \cdots \mathrm{F}_{2}$ & -434.875582109 & -0.002479161 & -0.000374298 & -0.000568484 & -0.003298068 \\
\hline $\mathrm{Cy} \cdots \mathrm{Cl}_{2}$ & -1154.991124929 & -0.004784002 & -0.000524045 & -0.001183904 & -0.006781053 \\
\hline $\mathrm{Cy} \cdots \mathrm{Br}_{2}$ & -5381.071058885 & -0.006303334 & -0.000874027 & -0.001639439 & -0.009790674 \\
\hline $\mathrm{Cy} \cdots \mathbf{I}_{2}$ & -825.471342047 & -0.006953214 & -0.001186844 & -0.001935467 & -0.011516278 \\
\hline $\mathrm{Cy} \cdots \mathrm{CH}_{4}$ & -275.962525140 & -0.002897061 & -0.000110561 & -0.000571797 & -0.003771304 \\
\hline
\end{tabular}

* SP - strong pairs; WP - weak pairs.

Table S5. Total electronic energies and dispersion part (a.u.) of cyclohexane $\cdots X$ dimers $\left(X=F_{2}, \mathrm{Cl}_{2}, \mathrm{Br}_{2}, \mathrm{I}_{2}, \mathrm{CH}_{4}\right)$

\begin{tabular}{|c|c|c|c|c|c|c|c|c|c|}
\hline & \multirow{2}{*}{ B3LYP } & \multirow{2}{*}{ D4 } & \multirow{2}{*}{ DD3S } & \multicolumn{2}{|c|}{ B3LYP-D3BJ } & \multicolumn{2}{|c|}{ CAM-B3LYP-D3BJ } & \multicolumn{2}{|c|}{ PBE0-D3BJ } \\
\hline & & & & $\mathrm{E}$ & D & E & D & E & D \\
\hline $\mathrm{Cy}_{\mathbf{W}} \mathrm{F}_{2}$ & -435.570956 & -0.024439 & -0.14766591 & -435.598988 & -0.028032 & -435.387029 & -0.012656 & -435.093848 & -0.014536 \\
\hline $\mathrm{Cy} \cdots \mathrm{Cl}_{2}$ & -1156.392815 & -0.028826 & -0.15994697 & -1156.425420 & -0.032605 & -1156.255103 & -0.015311 & -1155.777741 & -0.017031 \\
\hline $\mathrm{Cy} \cdots \mathrm{Br}_{2}$ & -5384.328933 & -0.030766 & -0.16360157 & -5384.363987 & -0.035053 & -5384.389099 & -0.016770 & -5383.395957 & -0.018227 \\
\hline $\mathrm{Cy} \cdots \mathrm{I}_{2}$ & -831.594235 & -0.033204 & -0.16681687 & -831.632178 & -0.037943 & -831.277324 & -0.018644 & -831.151068 & -0.019631 \\
\hline $\mathrm{Cy} \cdots \mathrm{CH}_{4}$ & -276.509209 & -0.027694 & -0.16571555 & -276.540466 & -0.031257 & -276.339681 & -0.014420 & -276.162562 & -0.016459 \\
\hline
\end{tabular}




\begin{tabular}{|c|c|c|c|c|c|c|c|c|c|}
\hline$C y$ from $C_{y} \cdots F_{2}$ & -235.971485 & -0.023433 & -0.14181370 & -235.997649 & -0.026164 & -235.832327 & -0.011523 & -235.684232 & -0.013359 \\
\hline Cy fro & -235.971521 & -0 & -0.14172863 & -235.997672 & 52 & -235.832344 & 20 & 53 & 354 \\
\hline Cy from $\mathrm{Cy} \cdots \mathrm{Br}_{2}$ & 34 & 0 & 70 & 79 & 45 & -235 & .011517 & 259 & .013351 \\
\hline Cy & 29 & -0.023419 & 47 & 76 & 42 & 51 & 517 & -235.684264 & -0.013350 \\
\hline $\mathrm{y} \cdots \mathrm{CH}_{4}$ & -235.9 & -0.023440 & -0.14187926 & -235.997626 & -0.026174 & -235.832301 & -0.011526 & -235.684217 & -0.013363 \\
\hline$F_{2}$ from $C_{y} \cdots F_{2}$ & -199.5 & -0.000 & -0.002 & -199 & -0.00 & -199 & -0.000104 & -199. & -0.000132 \\
\hline $\mathrm{Cl}_{2}$ from $\mathrm{Cy} \cdots \mathrm{Cl}_{2}$ & 20.422076 & -0.001858 & -0.00986 & -920.424 & -0.00 & $-920.41 c$ & -0.00 & -920.090246 & -0.000925 \\
\hline $\mathrm{Br}_{2}$ from $\mathrm{Cy} \cdots \mathrm{Br}_{2}$ & -5148.359147 & -0.002485 & -0.00939456 & -5148.361923 & -0.002776 & -5148.553625 & -0.001334 & -5147.707765 & -0.001224 \\
\hline$I_{2}$ from $\mathrm{Cy} \cdot \cdots I_{2}$ & -595 & -0. & 0.0 & -595 & -0.0 & -595 & -0.0 & -595 & -0.00 \\
\hline $\mathrm{CH}_{4}$ from $\mathrm{Cy} \cdots \mathrm{CH}_{4}$ & -40.539111 & -0.001389 & -0.01869869 & -40.541025 & -0.001914 & -40.505565 & -0.000718 & -40.476313 & -0.000922 \\
\hline
\end{tabular}

\begin{tabular}{|c|c|c|c|c|c|c|c|c|}
\hline & \multicolumn{2}{|c|}{ APFD } & \multicolumn{2}{|c|}{ B97d3 } & \multicolumn{2}{|c|}{ wb97xd } & \multicolumn{2}{|c|}{ TPSS-D3BJ } \\
\hline & $\mathrm{E}$ & D & $E$ & D & $\mathrm{E}$ & D & $\mathrm{E}$ & D \\
\hline$C_{y} \cdots F_{2}$ & -435.227088 & -0.013298 & -435.326890 & -0.048066 & -435.440627 & -0.013479 & -435.643818 & -0.021087 \\
\hline $\mathrm{Cy} \cdots \mathrm{Cl}_{2}$ & -1155.955066 & -0.014838 & -1156.269419 & -0.053650 & -1156.286891 & -0.014059 & -1156.462023 & -0.024533 \\
\hline $\mathrm{Cy} \cdots \mathrm{Br}_{2}$ & -5383.735183 & -0.016268 & -5386.761674 & -0.055844 & -5384.322564 & -0.014873 & -5384.147327 & -0.026186 \\
\hline $\mathrm{Cy} \cdots \mathrm{I}_{2}$ & -831.337669 & -0.016751 & -832.289469 & -0.057929 & -831.490373 & -0.015597 & -831.243626 & -0.028060 \\
\hline $\mathrm{Cy} \cdots \mathrm{CH}_{4}$ & -276.266368 & -0.014118 & -276.328504 & -0.053560 & -276.421660 & -0.015000 & -276.566341 & -0.023723 \\
\hline Cy from $C_{y} \cdots F_{2}$ & -235.769634 & -0.011012 & -235.814064 & -0.045309 & -235.898432 & -0.011607 & -236.021745 & -0.019512 \\
\hline $\mathrm{Cy}$ from $\mathrm{Cy} \cdots \mathrm{Cl}_{2}$ & -235.769653 & -0.011002 & -235.814093 & -0.045282 & -235.898447 & -0.011599 & -236.021762 & -0.019504 \\
\hline Cy from $\mathrm{Cy} \cdots \mathrm{Br}_{2}$ & -235.769655 & -0.010995 & -235.814098 & -0.045267 & -235.898454 & -0.011594 & -236.021755 & -0.019499 \\
\hline Cy from $\mathrm{Cy} \cdots \mathrm{I}_{2}$ & -235.769658 & -0.010992 & -235.814101 & -0.045262 & -235.898450 & -0.011592 & -236.021751 & -0.019497 \\
\hline $\mathrm{Cy}$ from $\mathrm{Cy} \cdots \mathrm{CH}_{4}$ & -235.769621 & -0.011022 & -235.814049 & -0.045332 & -235.898411 & -0.011614 & -236.021744 & -0.019519 \\
\hline$F_{2}$ from $C_{y} \cdots F_{2}$ & -199.455405 & 0.000000 & -199.511250 & -0.000570 & -199.541558 & -0.000004 & -199.620139 & -0.000199 \\
\hline $\mathrm{Cl}_{2}$ from $\mathrm{Cy} \cdots \mathrm{Cl}_{2}$ & -920.182030 & 0.000000 & -920.451537 & -0.002704 & -920.386592 & -0.000013 & -920.436786 & -0.001347 \\
\hline $\mathrm{Br}_{2}$ from $\mathrm{Cy} \cdots \mathrm{Br}_{2}$ & -5147.961033 & 0.000000 & -5150.942738 & -0.002889 & -5148.421768 & -0.000034 & -5148.120912 & -0.001729 \\
\hline$I_{2}$ from $C_{y} \cdots I_{2}$ & -595.563248 & 0.000000 & -596.469569 & -0.003466 & -595.589128 & -0.000084 & -595.216234 & -0.002459 \\
\hline $\mathrm{CH}_{4}$ from $\mathrm{Cy} \cdots \mathrm{CH}_{4}$ & -40.494607 & 0.000000 & -40.512386 & -0.004094 & -40.520933 & -0.000391 & -40.542762 & -0.001396 \\
\hline
\end{tabular}


Table S6. DLPNO-LED analysis (energies in a.u.) of Cy-R (Measure A)

\begin{tabular}{|c|c|c|c|c|c|}
\hline & $E^{\text {tot }}$ & $E^{\mathrm{disp}}(\mathrm{SP})$ & $E^{\text {disp }}(\mathrm{WP})$ & inter Triples & inter SP total \\
\hline $1 F_{\text {eq }}$ & -334.681248461 & -0.012734721 & -0.000603725 & -0.006316868 & -0.045351840 \\
\hline $1 F_{\mathrm{ax}}$ & -334.681023434 & -0.013596564 & -0.000600815 & -0.006491247 & -0.046805721 \\
\hline $1 \mathrm{Cl}_{\mathrm{eq}}$ & -694.676049963 & -0.016883657 & -0.000548656 & -0.008529235 & -0.060022757 \\
\hline $1 \mathrm{Clax}$ & -694.675430195 & -0.018300390 & -0.000565710 & -0.008869269 & -0.062433088 \\
\hline $1 \mathrm{Br}_{\mathrm{eq}}$ & -2807.706080511 & -0.018732423 & -0.000845763 & -0.008745086 & -0.062922071 \\
\hline $1 \mathrm{Br}_{\mathrm{ax}}$ & -2807.705845196 & -0.021274727 & -0.000958935 & -0.009132302 & -0.065650864 \\
\hline $1 \mathrm{l}_{\text {eq }}$ & -529.891837241 & -0.019432184 & -0.000878025 & -0.009413971 & -0.067166299 \\
\hline $1 l_{\mathrm{ax}}$ & -529.891875753 & -0.022859648 & -0.000925340 & -0.009860256 & -0.070307411 \\
\hline $1 \mathrm{CCH}_{\text {eq }}$ & -311.533556644 & -0.025374066 & -0.000654160 & -0.008582583 & -0.063825960 \\
\hline $1 \mathrm{CCH}_{\mathrm{ax}}$ & -311.533151278 & -0.026880881 & -0.000659741 & -0.008981782 & -0.066607057 \\
\hline $1 \mathrm{CN}_{\mathrm{eq}}$ & -327.630758227 & -0.022526216 & -0.000647323 & -0.008406408 & -0.062138460 \\
\hline $1 \mathrm{CN}_{\mathrm{ax}}$ & -327.630820176 & -0.024454371 & -0.000668296 & -0.008785910 & -0.064742422 \\
\hline $1 \mathrm{Me}_{\text {eq }}$ & -274.764309092 & -0.022363900 & -0.000415606 & -0.006557106 & -0.058993429 \\
\hline 1 Meax $_{\text {ax }}$ & -274.761484653 & -0.023674178 & -0.000434617 & -0.006807183 & -0.060903031 \\
\hline 1Et $\mathrm{teq}_{\mathrm{A}} \mathrm{A}$ & -314.015617126 & -0.027406120 & -0.000702000 & -0.007761226 & -0.064676917 \\
\hline 1Et $\mathrm{eq}_{\text {eq }}$ B & -314.014306846 & -0.027652119 & -0.000658291 & -0.007818160 & -0.065018778 \\
\hline $1 \mathrm{Et}_{\mathrm{ax}} \mathrm{C}$ & -314.013002298 & -0.028477590 & -0.000675916 & -0.008093405 & -0.067149036 \\
\hline $1 \mathrm{Et}_{\mathrm{ax}}\left(C_{s}\right)$ & -314.006801417 & -0.029090789 & -0.000632980 & -0.008116880 & -0.067041447 \\
\hline $1 \mathrm{iPr}$ eq $\mathrm{A}$ & -353.267941215 & -0.032832153 & -0.001063573 & -0.009336695 & -0.072198147 \\
\hline \multicolumn{6}{|l|}{$1 \mathrm{iPr}_{\mathrm{eq}} \mathrm{B}$} \\
\hline $1 \mathrm{iPr}_{\mathrm{ax}} \mathrm{C}$ & -353.265726890 & -0.032180664 & -0.000912742 & -0.009486477 & -0.074169386 \\
\hline $1 \mathrm{tBu}_{\mathrm{eq}}$ & -392.521485059 & -0.035209004 & -0.001278722 & -0.010150124 & -0.076445023 \\
\hline $1 \mathrm{tBu} \mathrm{ax}_{\mathrm{x}}$ & -392.513711459 & -0.037625428 & -0.001211192 & -0.010801941 & -0.080481220 \\
\hline $1 \mathrm{Ph}_{\text {eq }} \mathrm{A}$ & -466.194269922 & -0.029632912 & -0.001413558 & -0.009707489 & -0.070598345 \\
\hline $1 \mathrm{Ph}_{\mathrm{ax}} \mathrm{B}$ & -466.189927114 & -0.032420509 & -0.001435609 & -0.010382553 & -0.075037127 \\
\hline $1 \mathrm{Ph}$ ax $\mathrm{C}$ & -466.187775494 & -0.032281398 & -0.001446301 & -0.010129799 & -0.073328876 \\
\hline $1 \mathrm{CF}_{3 \mathrm{eq}}$ & -572.297296804 & -0.022578089 & -0.001512991 & -0.007888697 & -0.056295287 \\
\hline
\end{tabular}




\begin{tabular}{|c|c|c|c|c|c|}
\hline $1 \mathrm{CF}_{3 \mathrm{ax}}$ & -572.293567933 & -0.023681558 & -0.001250187 & -0.008245555 & -0.058733130 \\
\hline $1 \mathrm{CCl}_{3 \text { eq }}$ & -1652.235554979 & -0.032017888 & -0.001216075 & -0.011138072 & -0.078553366 \\
\hline $1 \mathrm{CCl}_{3 \mathrm{ax}}$ & -1652.227853496 & -0.032960852 & -0.001193836 & -0.011514417 & -0.081002230 \\
\hline $1 \mathrm{CBr}_{3 \mathrm{eq}}$ & -7991.321300610 & -0.037133215 & -0.001922057 & -0.012635990 & -0.083696872 \\
\hline $1 \mathrm{CBr}_{3 \mathrm{ax}}$ & -7991.312907140 & -0.037948911 & -0.001967867 & -0.013091741 & -0.086636367 \\
\hline $1 \mathrm{Cl}_{3 \mathrm{eq}}$ & -1157.880161184 & -0.038577856 & -0.002025907 & -0.014015462 & -0.090260354 \\
\hline $1 \mathrm{Cl}_{3 \mathrm{ax}}$ & -1157.870523775 & -0.040572803 & -0.002151172 & -0.014462731 & -0.093081782 \\
\hline $1 \mathrm{SiMe}_{\text {зеq }}$ & -643.580158328 & -0.017867836 & -0.001046383 & -0.005946253 & -0.036202845 \\
\hline $1 \mathrm{SiMe}_{3 \mathrm{ax}}$ & -643.576492913 & -0.019956525 & -0.000929565 & -0.006435306 & -0.039352837 \\
\hline
\end{tabular}

* SP - strong pairs; WP - weak pairs.

Table S7. DLPNO-LED analysis (energies in a.u.) of Cy-R (Measure B)

\begin{tabular}{|c|c|c|c|c|c|}
\hline & $E^{\text {tot }}$ & $E^{\text {disp }}(\mathrm{SP})$ & $E^{\text {disp }}$ (WP) & inter Triples & inter SP total \\
\hline $1 F_{\mathrm{eq}}$ & -297.491642026 & -0.014386355 & -0.000603428 & -0.004568801 & -0.033563636 \\
\hline $1 F_{\mathrm{ax}}$ & -297.498860397 & -0.015436890 & -0.000619794 & -0.004759740 & -0.035060886 \\
\hline $1 \mathrm{Cl}_{\mathrm{eq}}$ & -657.508958880 & -0.018906843 & -0.000550507 & -0.006062347 & -0.042017702 \\
\hline $1 \mathrm{Cl}_{\mathrm{ax}}$ & -657.518821579 & -0.020248079 & -0.000588776 & -0.006403906 & -0.044359384 \\
\hline $1 \mathrm{Br}_{\mathrm{eq}}$ & -2770.543951962 & -0.020052833 & -0.000889757 & -0.006164777 & -0.043914304 \\
\hline $1 \mathrm{Br}_{\mathrm{ax}}$ & -2770.554611960 & -0.021132894 & -0.001007670 & -0.006546049 & -0.046562964 \\
\hline $1 l_{\text {eq }}$ & -492.733922209 & -0.021395075 & -0.000917184 & -0.006705517 & -0.047139529 \\
\hline $1 l_{a x}$ & -492.745163959 & -0.022724975 & -0.000943405 & -0.007135249 & -0.050139571 \\
\hline $1 \mathrm{CCH}_{\mathrm{eq}}$ & -274.256214096 & -0.022124568 & -0.000671861 & -0.007191055 & -0.049674716 \\
\hline $1 \mathrm{CCH}_{\mathrm{ax}}$ & -274.262891320 & -0.024339484 & -0.000690413 & -0.007615305 & -0.052448543 \\
\hline $1 \mathrm{CN}_{\mathrm{eq}}$ & -290.355769405 & -0.020682143 & -0.000668428 & -0.006879188 & -0.047338376 \\
\hline $1 \mathrm{CN}_{\mathrm{ax}}$ & -290.360051472 & -0.022821452 & -0.000704558 & -0.007307376 & -0.050096893 \\
\hline $1 \mathrm{Meq}_{\mathrm{eq}}$ & -237.520178973 & -0.022288323 & -0.000589273 & -0.006348226 & -0.049472567 \\
\hline $1 \mathrm{Me}_{\mathrm{ax}}$ & -237.533876461 & -0.023540436 & -0.000610340 & -0.006527040 & -0.050704423 \\
\hline 1Eteq $A$ & -276.776581211 & -0.024130083 & -0.000835077 & -0.006974233 & -0.052584830 \\
\hline
\end{tabular}




\begin{tabular}{|c|c|c|c|c|c|}
\hline 1Eteq B & -276.785109125 & -0.024088752 & -0.000757215 & -0.006996585 & -0.052592261 \\
\hline $1 E t_{a x} C$ & -276.788561873 & -0.025365772 & -0.000795066 & -0.007226084 & -0.054319122 \\
\hline $1 \mathrm{Et}_{\mathrm{ax}}\left(C_{\mathrm{s}}\right)$ & -276.808006764 & -0.025204065 & -0.000759081 & -0.007097448 & -0.053031458 \\
\hline $1 \mathrm{iPr}_{\text {eq }} \mathrm{A}$ & -316.041496346 & -0.026324036 & -0.001087947 & -0.007609945 & -0.055739896 \\
\hline $1 \mathrm{iPr} r_{a x} \mathrm{C}$ & -316.049280266 & -0.027445932 & -0.000979501 & -0.007913781 & -0.057819948 \\
\hline $1 \mathrm{tBu}$ eq & -355.312536924 & -0.027036830 & -0.001252419 & -0.008124198 & -0.058014393 \\
\hline $1 \mathrm{tBu}$ ax & -355.332302206 & -0.029040868 & -0.001168025 & -0.008305933 & -0.059024376 \\
\hline $1 \mathrm{Ph}_{\mathrm{eq}} \mathrm{A}$ & -428.951222189 & -0.026212695 & -0.001357498 & -0.008066130 & -0.054839750 \\
\hline $1 \mathrm{Ph}_{\mathrm{ax}} \mathrm{B}$ & -428.968999852 & -0.027864963 & -0.001423558 & -0.008635201 & -0.058373768 \\
\hline $1 \mathrm{Ph}_{\mathrm{ax}} \mathrm{C}$ & -428.972373840 & -0.025915371 & -0.001417171 & -0.008243802 & -0.055646604 \\
\hline $1 \mathrm{CF}_{3 \mathrm{eq}}$ & -535.040139889 & -0.022381635 & -0.001377458 & -0.006799602 & -0.049922420 \\
\hline $1 \mathrm{CF}_{3 \mathrm{ax}}$ & -535.056715328 & -0.023940230 & -0.001162408 & -0.006960356 & -0.050895799 \\
\hline $1 \mathrm{CCl}_{3 \mathrm{eq}}$ & -1615.008604984 & -0.027474560 & -0.001178810 & -0.009075534 & -0.060227285 \\
\hline $1 \mathrm{CCl}_{3 \mathrm{ax}}$ & -1615.031189503 & -0.028435436 & -0.001165461 & -0.009220394 & -0.060910713 \\
\hline $1 \mathrm{CBr}_{3 \mathrm{eq}}$ & -7954.093535680 & -0.029129710 & -0.001780181 & -0.009543232 & -0.063482936 \\
\hline $1 \mathrm{CBr}_{3 \mathrm{ax}}$ & -7954.118491180 & -0.030903975 & -0.001864490 & -0.009679290 & -0.064170710 \\
\hline $1 \mathrm{Cl}_{\text {3eq }}$ & -1120.657871516 & -0.031772367 & -0.001947663 & -0.010469820 & -0.067785751 \\
\hline $1 \mathrm{Cl}_{3 \mathrm{ax}}$ & -1120.685987567 & -0.031528483 & -0.002028931 & -0.010566303 & -0.068242138 \\
\hline 1SiMe & -606.328962584 & -0.027585991 & -0.001115317 & -0.008122255 & -0.059946650 \\
\hline $1 \mathrm{SiMe}_{3 \mathrm{ax}}$ & -606.349165746 & -0.028569090 & -0.001054551 & -0.008295341 & -0.060684760 \\
\hline
\end{tabular}

* SP - strong pairs; WP - weak pairs.

Table S8. Single point energies (in a.u.) of $\mathrm{Cy}-\mathrm{R}$, and of $\mathrm{Cy}-\mathrm{H}, \mathrm{H}-\mathrm{R}$ at the geometries of the full molecule, computed at HF/aug-cc-pVQZ and DLPNO-CCSD(T)/aug-cc-pVQZ level of theory (the aug-cc-pVQZ-PP basis set was used for iodine). From MP2/aug-cc-pVTZ optimized geometries.

\begin{tabular}{|c|c|c|c|c|c|c|c|}
\hline & $E_{\mathrm{Cy}-\mathrm{R}} \mathrm{HF}$ & $E_{\mathrm{C} y-\mathrm{R}}$ DLPNO & $E_{\mathrm{Cy}-\mathrm{H}} \mathrm{HF}$ & $E_{\mathrm{C} y-\mathrm{H}}$ DLPNO & $E_{\mathrm{H}-\mathrm{R}} \mathrm{HF}$ & $E_{\mathrm{H}-\mathrm{R}}$ DLPNO & $\begin{array}{c}\text { Interfragment correlation energy } \\
\text { stabilization }\left(\mathrm{kcal} \mathrm{mol}^{-1}\right)\end{array}$ \\
\hline $1 F_{\mathrm{eq}}$ & -333.2058134 & -334.6812485 & -234.3001058 & -235.5080924 & & & \\
\hline $1 F_{a x}$ & -333.2053559 & -334.6810234 & -234.3003961 & -235.5081797 & & & -0.27 \\
\hline $1 \mathrm{Cl}_{\text {eq }}$ & -693.2476363 & -694.6760500 & -234.3003475 & -235.5083084 & & & \\
\hline
\end{tabular}




\begin{tabular}{|c|c|c|c|c|c|c|c|}
\hline $1 \mathrm{Clax}_{\mathrm{ax}}$ & -693.2455483 & -694.6754302 & -234.3004470 & -235.5081587 & & & -1.08 \\
\hline $1 \mathrm{Br}_{\mathrm{eq}}$ & -2806.1887920 & -2807.7060805 & -234.3002382 & -235.5081915 & & & \\
\hline $1 \mathrm{Br}_{\mathrm{ax}}$ & -2806.1861290 & -2807.7058452 & -234.3002856 & -235.5080011 & & & -1.67 \\
\hline $11_{\text {eq }}$ & -528.3824343 & -529.8918372 & -234.3002507 & -235.5082246 & & & \\
\hline $1 \mathrm{lax}_{\mathrm{ax}}$ & -528.3792501 & -529.8918758 & -234.3000423 & -235.5077964 & & & -2.16 \\
\hline $1 \mathrm{CCH}_{\mathrm{eq}}$ & -310.0010925 & -311.5335566 & -234.3004675 & -235.5086843 & -76.8522046 & -77.2099125 & \\
\hline $1 \mathrm{CCH}_{\mathrm{ax}}$ & -309.9987446 & -311.5331513 & -234.3004339 & -235.5085047 & -76.8521616 & -77.2099153 & -1.28 \\
\hline $1 \mathrm{CN}_{\mathrm{eq}}$ & -326.0672622 & -327.6307582 & -234.3006441 & -235.5087541 & -92.9105997 & -93.3019636 & \\
\hline $1 \mathrm{CN}_{\mathrm{ax}}$ & -326.0655809 & -327.6308202 & -234.3006884 & -235.5086611 & -92.9105346 & -93.3019458 & -1.15 \\
\hline $1 \mathrm{Me}_{\mathrm{eq}}$ & -273.3519893 & -274.7643091 & -234.3003834 & -235.5086384 & -40.2158243 & -40.4514959 & \\
\hline $1 \mathrm{Me}_{\mathrm{ax}}$ & -273.3478182 & -274.7614847 & -234.3000061 & -235.5079656 & -40.2157476 & -40.4514086 & -1.04 \\
\hline 1Eteq A & -312.3999295 & -314.0156171 & -234.3003238 & -235.5085838 & -79.2646255 & -79.6999650 & \\
\hline 1Eteq $B$ & -312.3978920 & -314.0143068 & -234.2998379 & -235.5082902 & -79.2645210 & -79.6998614 & \\
\hline $1 \mathrm{Et}_{\mathrm{ax}} \mathrm{C}$ & -312.3954551 & -314.0130023 & -234.2998123 & -235.5078546 & -79.2644760 & -79.6998154 & -1.30 \\
\hline $1 \mathrm{Et}_{\mathrm{ax}}\left(C_{s}\right)$ & -312.3888664 & -314.0068014 & -234.2954659 & -235.5035470 & -79.2633140 & -79.6987821 & \\
\hline $1 \mathrm{iPr}$ eq $\mathrm{A}$ & -351.4458987 & -353.2679412 & -234.3001609 & -235.5083758 & -118.3140243 & -118.9513532 & \\
\hline $1 \mathrm{iPr} \mathrm{eq}_{\mathrm{e}} \mathrm{B}$ & -351.4458414 & -353.2679047 & -234.2996190 & -235.5081288 & -118.3144741 & -118.9516430 & \\
\hline $1 \mathrm{iPr}_{\mathrm{ax}} \mathrm{C}$ & -351.4413550 & -353.2657269 & -234.2991748 & -235.5074219 & -118.3141036 & -118.9513776 & -1.48 \\
\hline $1 \mathrm{tBu}$ eq & -390.4908441 & -392.5214851 & -234.2992164 & -235.5077598 & -157.3640203 & -158.2054060 & \\
\hline $1 \mathrm{tBu} \mathrm{ux}_{\mathrm{ax}}$ & -390.4804242 & -392.5137115 & -234.2949546 & -235.5032327 & -157.3627092 & -158.2044039 & -1.63 \\
\hline $1 \mathrm{Ph}_{\mathrm{eq}} \mathrm{A}$ & -463.9300156 & -466.1942699 & -234.3003755 & -235.5086447 & -230.7937690 & -231.8746541 & \\
\hline $1 \mathrm{Ph}$ ax $\mathrm{B}$ & -463.9215424 & -466.1899271 & -234.2990559 & -235.5072820 & -230.7932380 & -231.8741693 & -2.59 \\
\hline $1 \mathrm{Ph}_{\mathrm{ax}} \mathrm{C}$ & -463.9210387 & -466.1877755 & -234.2976568 & -235.5055377 & -230.7928285 & -231.8738887 & \\
\hline $1 \mathrm{CF}_{3 \mathrm{eq}}$ & -570.0830476 & -572.2972968 & -234.3007306 & -235.5087569 & -336.9319992 & -337.9687414 & \\
\hline $1 \mathrm{CF}_{3 \mathrm{ax}}$ & -570.0779940 & -572.2935679 & -234.2990292 & -235.5066456 & -336.9315058 & -337.9683555 & -1.02 \\
\hline $1 \mathrm{CCl}_{3 \mathrm{eq}}$ & -1650.1499551 & -1652.2355550 & -234.3001258 & -235.5083456 & -1417.0126742 & -1417.9089921 & \\
\hline $1 \mathrm{CCl}_{3 \mathrm{ax}}$ & -1650.1399407 & -1652.2278535 & -234.2956150 & -235.5034674 & -1417.0114560 & -1417.9079839 & -1.55 \\
\hline $1 \mathrm{CBr}_{3 \mathrm{eq}}$ & -7988.9661905 & -7991.3213006 & -234.2999713 & -235.5082453 & -7755.8337335 & -7756.9886630 & \\
\hline $1 \mathrm{CBr}_{3 a x}$ & -7988.9544204 & -7991.3129071 & -234.2950146 & -235.5029115 & -7755.8321343 & -7756.9873341 & -2.19 \\
\hline $1 \mathrm{Cl}_{\text {3eq }}$ & -1155.5468774 & -1157.8801612 & -234.2997323 & -235.5081076 & -922.4206429 & -923.5454173 & \\
\hline
\end{tabular}




\begin{tabular}{|c|c|c|c|c|c|c|c|}
\hline $1 \mathrm{Cl}_{3 a x}$ & -1155.5331814 & -1157.8705238 & -234.2938450 & -235.5018947 & -922.4183645 & -923.5435622 & -2.49 \\
\hline 1SiMe & -641.5981999 & -643.5801583 & -234.3002472 & -235.5085821 & -408.4587972 & -409.2544893 & \\
\hline $1 \mathrm{SiMe}_{3 \mathrm{ax}}$ & -641.5918234 & -643.5764929 & -234.2973027 & -235.5055787 & -408.4573264 & -409.2533064 & -1.56 \\
\hline
\end{tabular}

* Computed with the following keyword line: ! dlpno-ccsd(t) aug-cc-pvqz aug-cc-pvqz/c aug-cc-pv5z/jk rijk verytightscf TightPNO

Table S9. Dispersion correction from single point energies (in a.u.) of $\mathrm{Cy}-\mathrm{R}$, and of $\mathrm{Cy}-\mathrm{H}, \mathrm{H}-\mathrm{R}$ at the geometries of the full molecule, computed at the B3LYP-D3BJ/def2TZVPP and DD3S/def2-TZVPP levels of theory and . From MP2/aug-cc-pVTZ optimized geometries.

\begin{tabular}{|c|c|c|c|c|c|c|c|c|}
\hline & D3BJ $\mathrm{c}_{y-\mathrm{R}}$ & $\mathrm{D} 3 \mathrm{BJ} \mathrm{Cy}_{\mathrm{H}} \mathrm{H}$ & $\mathrm{D} \mathrm{BJ}_{\mathrm{H}-\mathrm{R}}$ & $\begin{array}{l}-\Delta E^{\text {disp }}{ }_{\text {ax-eq }}(C y \cdots R) \text { from } \\
\text { B3LYP-D3BJ }\left(k c a l ~ \mathrm{~mol}^{-1}\right)\end{array}$ & ${ }^{D D} 3 S_{C y-R}$ & $\mathrm{DD}_{3} \mathrm{~S}_{\mathrm{y}-\mathrm{H}}$ & $\mathrm{DD} \mathrm{S}_{\mathrm{H}-\mathrm{R}}$ & $\begin{array}{c}-\Delta E^{\text {disp }}{ }_{a x-e q}(C y \cdots R) \text { from } \\
\text { DD3S }\left(k \mathrm{kcal} \mathrm{mol}^{-1}\right)\end{array}$ \\
\hline $1 F_{\text {eq }}$ & -0.0266570 & -0.0262554 & & & -0.1430714 & -0.1426749 & & \\
\hline $1 F_{a x}$ & -0.0267587 & -0.0261931 & & -0.10 & -0.1430846 & -0.1422777 & & -0.26 \\
\hline $1 \mathrm{Cl}_{\text {eq }}$ & -0.0305470 & -0.0262306 & & & -0.1541427 & -0.1424134 & & \\
\hline $1 \mathrm{Clax}_{\mathrm{ax}}$ & -0.0316370 & -0.0261384 & & -0.74 & -0.1564350 & -0.1418600 & & -1.79 \\
\hline $1 \mathrm{Br}_{\mathrm{eq}}$ & -0.0317395 & -0.0262282 & & & -0.1555835 & -0.1423976 & & \\
\hline $1 \mathrm{Br}_{\mathrm{ax}}$ & -0.0331798 & -0.0261370 & & -0.96 & -0.1587422 & -0.1418633 & & -2.32 \\
\hline $11_{\text {eq }}$ & -0.0334382 & -0.0262139 & & & -0.1574072 & -0.1422484 & & \\
\hline $1 l_{a x}$ & -0.0353162 & -0.0261137 & & -1.24 & -0.1612512 & -0.1416842 & & -2.77 \\
\hline $1 \mathrm{CCH}_{\mathrm{eq}}$ & -0.0323981 & -0.0261907 & -0.0022630 & & -0.1655471 & -0.1419384 & -0.0162451 & \\
\hline $1 \mathrm{CCH}_{\mathrm{ax}}$ & -0.0336991 & -0.0261424 & -0.0022628 & -0.85 & -0.1681645 & -0.1416311 & -0.0162418 & -1.84 \\
\hline $1 \mathrm{CN}_{\mathrm{eq}}$ & -0.0312859 & -0.0261899 & -0.0014531 & & -0.1600569 & -0.1419458 & -0.0111141 & \\
\hline $1 \mathrm{CN}_{\mathrm{ax}}$ & -0.0325083 & -0.0261411 & -0.0014531 & -0.80 & -0.1626921 & -0.1416274 & -0.0111133 & -1.85 \\
\hline $1 \mathrm{Me}_{\mathrm{eq}}$ & -0.0321690 & -0.0261944 & -0.0019134 & & -0.1691880 & -0.1420364 & -0.0186503 & \\
\hline $1 \mathrm{Me}_{\mathrm{ax}}$ & -0.0331477 & -0.0260654 & -0.0019134 & -0.70 & -0.1712366 & -0.1412191 & -0.0186573 & -1.79 \\
\hline $1 \mathrm{Et}_{\text {eq }} \mathrm{A}$ & -0.0377809 & -0.0261863 & -0.0054197 & & -0.1956051 & -0.1419679 & -0.0406075 & \\
\hline $1 \mathrm{Et}_{\mathrm{eq}} \mathrm{B}$ & -0.0383500 & -0.0262263 & -0.0054183 & & -0.1967192 & -0.1422127 & -0.0405919 & \\
\hline $1 \mathrm{Et}_{\mathrm{ax}} \mathrm{C}$ & -0.0391132 & -0.0260765 & -0.0054228 & -0.90 & -0.1984819 & -0.1412625 & -0.0406584 & -2.22 \\
\hline $1 \mathrm{Et}_{\mathrm{ax}}\left(C_{\mathrm{s}}\right)$ & -0.0394660 & -0.0258483 & -0.0054113 & & -0.1977837 & -0.1403101 & -0.0405419 & \\
\hline $1 \mathrm{iPr}_{\text {eq }} \mathrm{A}$ & -0.0447058 & -0.0261620 & -0.0100178 & & -0.2257852 & -0.1417935 & -0.0653327 & \\
\hline $1 \mathrm{iPr}_{\mathrm{eq}} \mathrm{B}$ & -0.0450816 & -0.0262297 & -0.0099946 & & -0.2259966 & -0.1422222 & -0.0651161 & \\
\hline $1 \mathrm{iPr}$ ax $\mathrm{C}$ & -0.0464398 & -0.0261087 & -0.0100148 & -1.12 & -0.2295489 & -0.1414415 & -0.0653609 & -2.57 \\
\hline $1 \mathrm{tBu}$ eq & -0.0531418 & -0.0262232 & -0.0156974 & & -0.2590502 & -0.1421544 & -0.0925684 & \\
\hline
\end{tabular}




\begin{tabular}{|c|c|c|c|c|c|c|c|c|}
\hline $1+B u_{a x}$ & -0.0547668 & -0.0258941 & -0.0157351 & -1.20 & -0.2621460 & -0.1405255 & -0.0928789 & -2.77 \\
\hline $1 \mathrm{Ph}_{\text {eq }} \mathrm{A}$ & -0.0534620 & -0.0261848 & -0.0189200 & & -0.2633231 & -0.1418927 & -0.1054692 & \\
\hline $1 \mathrm{Ph}_{\mathrm{ax}} \mathrm{B}$ & -0.0557830 & -0.0261142 & -0.0189202 & -1.50 & -0.2694972 & -0.1415406 & -0.1054745 & -4.09 \\
\hline $1 \mathrm{Ph}_{\mathrm{ax}} \mathrm{C}$ & -0.0550918 & -0.0258903 & -0.0189165 & & -0.2662911 & -0.1403469 & -0.1054348 & \\
\hline $1 \mathrm{CF}_{3 \mathrm{eq}}$ & -0.0337399 & -0.0262057 & -0.0026774 & & -0.1733906 & -0.1421161 & -0.0209621 & \\
\hline $1 \mathrm{CF}_{3 \mathrm{ax}}$ & -0.0345320 & -0.0259498 & -0.0026774 & -0.66 & -0.1743416 & -0.1406941 & -0.0209615 & -1.49 \\
\hline $1 \mathrm{CCl}_{3 \mathrm{eq}}$ & -0.0479860 & -0.0262353 & -0.0103207 & & -0.2118778 & -0.1422807 & -0.0457364 & \\
\hline $1 \mathrm{CCl}_{3 \mathrm{ax}}$ & -0.0494056 & -0.0258433 & -0.0103297 & -1.13 & -0.2134491 & -0.1403007 & -0.0457866 & -2.20 \\
\hline $1 \mathrm{CBr}_{3 \mathrm{eq}}$ & -0.0530269 & -0.0262489 & -0.0131049 & & -0.2180254 & -0.1423876 & -0.0475023 & \\
\hline $1 \mathrm{CBr}_{3 \mathrm{ax}}$ & -0.0546976 & -0.0258351 & -0.0131196 & -1.30 & -0.2199751 & -0.1403049 & -0.0475364 & -2.51 \\
\hline $1 \mathrm{Cl}_{\text {3eq }}$ & -0.0607590 & -0.0262540 & -0.0178616 & & -0.2262513 & -0.1424111 & -0.0514815 & \\
\hline $1 \mathrm{Cl}_{3 \mathrm{ax}}$ & -0.0626066 & -0.0258085 & -0.0178976 & -1.42 & -0.2280981 & -0.1402026 & -0.0515287 & -2.52 \\
\hline $1 \mathrm{SiMe}_{3 \mathrm{eq}}$ & -0.0544644 & -0.0261430 & -0.0176096 & & -0.2474103 & -0.1415262 & -0.0881860 & \\
\hline $1 \mathrm{SiMe}_{3 а x}$ & -0.0567517 & -0.0259879 & -0.0176799 & -1.49 & -0.2519943 & -0.1407284 & -0.0883916 & -3.25 \\
\hline
\end{tabular}




\section{Dispersion Stabilization and Steric Part}

Table S10. LD stabilizations in $\mathrm{kcal} \mathrm{mol}^{-1}$, from D3BJ computations. The uncorrected electronic energy $\Delta E_{\text {ax-eq }}^{\text {disp }}$ is the LD stabilization in the whole molecule; $\Delta E^{\text {disp }}{ }_{\text {ax-eq }}(C y)$ is the cyclohexyl correction computed from the cyclohexane ring in the axial and equatorial geometries; $\Delta E^{\text {disp }}{ }_{\text {ax-eq }}(R)$ is the correction for the $\mathrm{R}$ group (with an added hydrogen for the non-halogen groups) in the axial and equatorial geometries. The corrected $\Delta E^{\text {disp }}{ }_{\text {ax- }}$ eq $(\mathrm{Cy} \cdots \mathrm{R})$ is computed by subtracting the corrections from the uncorrected value.

\begin{tabular}{|c|c|c|c|c|}
\hline $\mathbf{R}$ & $\Delta E_{\text {ax-eq }}^{\text {disp }}$ & $\begin{array}{c}\Delta E_{\text {disp }}^{\text {dax-eq }} \\
(C y)\end{array}$ & $\begin{array}{l}\Delta E^{\text {disp }}{ }_{\text {ax-eq }} \\
\text { (R) }\end{array}$ & $\begin{array}{c}\Delta E_{\text {disp }}^{\text {ax-eq }} \\
(\text { (Cy } \cdots R)\end{array}$ \\
\hline$F$ & 0.06 & -0.04 & - & 0.10 \\
\hline $\mathrm{Cl}$ & 0.68 & -0.06 & - & 0.74 \\
\hline $\mathrm{Br}$ & 0.90 & -0.06 & - & 0.96 \\
\hline $\mathbf{I}$ & 1.18 & -0.06 & - & 1.24 \\
\hline $\mathrm{CCH}$ & 0.82 & -0.03 & 0.00 & 0.85 \\
\hline CN & 0.77 & -0.03 & 0.00 & 0.80 \\
\hline Me & 0.61 & -0.08 & 0.00 & 0.70 \\
\hline Et & 0.84 & -0.07 & 0.00 & 0.90 \\
\hline $\mathrm{iPr}$ & 1.09 & -0.03 & 0.00 & 1.12 \\
\hline $\mathrm{tBu}$ & 1.02 & -0.21 & 0.02 & 1.20 \\
\hline $\mathrm{Ph}$ & 1.46 & -0.04 & 0.00 & 1.50 \\
\hline $\mathrm{CF}_{3}$ & 0.50 & -0.16 & 0.00 & 0.66 \\
\hline $\mathrm{CCl}_{3}$ & 0.89 & -0.25 & 0.01 & 1.13 \\
\hline $\mathrm{CBr}_{3}$ & 1.05 & -0.26 & 0.01 & 1.30 \\
\hline $\mathrm{Cl}_{3}$ & 1.16 & -0.28 & 0.02 & 1.42 \\
\hline $\mathrm{SiMe}_{3}$ & 1.44 & -0.10 & 0.04 & 1.49 \\
\hline
\end{tabular}

The relationship between the dispersion stabilization and the polarizabilities of the various groups in shown in Fig. S7. For the smaller groups, this relationship is close to linear, but addition of larger groups makes the relationship more logarithmic in nature. The non-linearity could probably be explained by the fact that the cyclohexyl moiety interacts only with the part of the substituent group that is closest to it. As the larger groups tilt away from the ring, most of the group is farther away and the interactions are weaker, and only a small part of the group is responsible for the interaction. Another possible explanation is that larger groups cause more deformation of the cyclohexane ring in the axial conformer, increase atom-atom distances and thus decrease the LD stabilization (relative to the geometry without deformation, as with smaller groups). 
A

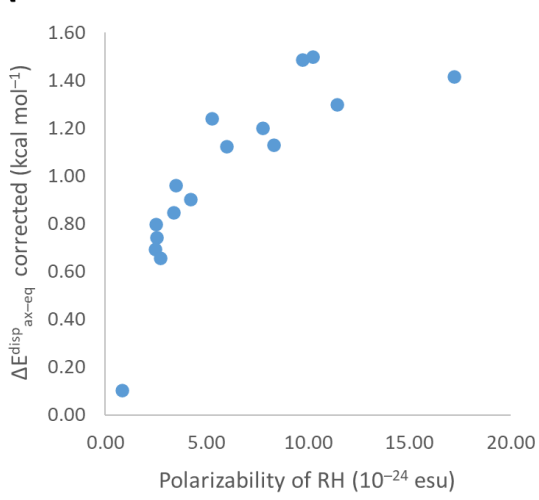

- D3BJ (B3LYP)

B

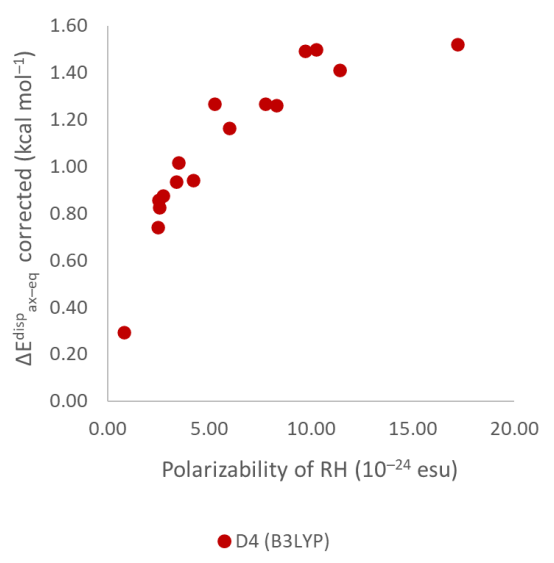

Figure S7. Plot of the LD energy from either the A) D3BJ or B) D4 correction against the computed polarizabilities of the $\mathrm{RH}$ molecules (formed from group $\mathrm{R}$ with an added hydrogen).

A

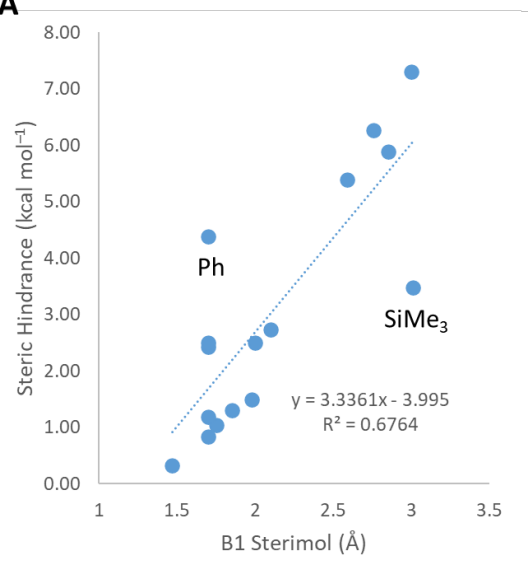

B

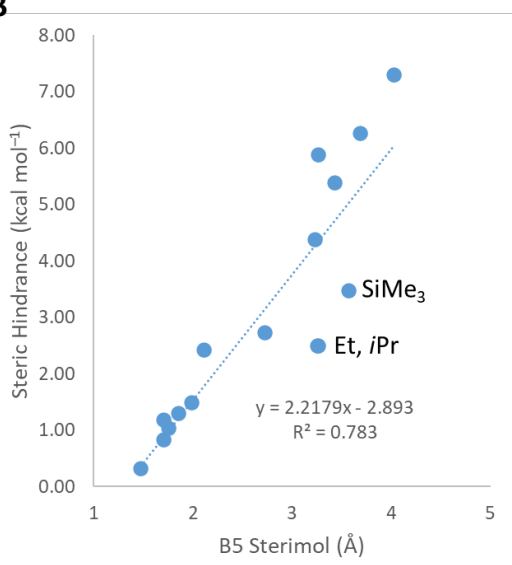

C

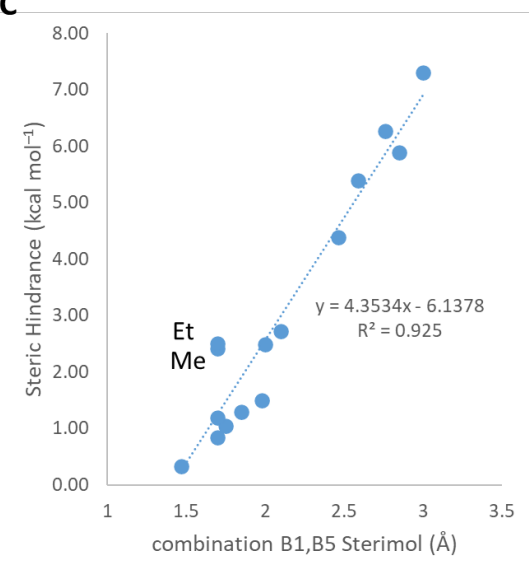

Figure S8. A correlation between the steric hindrance part (computed from subtraction of the corrected dispersion stabilization: $\left.\Delta E_{\text {ax-eq }}-\Delta E^{\text {disp }}{ }_{\text {ax-eq }}(C y \cdots \mathrm{R})\right)$ and the B1 Sterimol (A), B5 Sterimol (B), and a combination of $\mathrm{B} 1$ and $\mathrm{B} 5$ Sterimol parameters. The combination used was decided based on inspection of the orientations of the different groups: The B1 Sterimol parameter was used for all groups, except for Ph, for which the average between the B1 and B5 Sterimol parameters was taken. The $\mathrm{SiMe}_{3}$ group was taken out from the correlation. The relatively small steric part of this group can be explained by the increased $\mathrm{C}(\mathrm{Cy})-\mathrm{Si}$ and $\mathrm{Si}-$ $\mathrm{C}(\mathrm{Me})$ bond lengths. 


\section{Experimental A-values}

Table S11. Data used in analysis of experimental A-values

\begin{tabular}{|c|c|c|c|c|c|c|c|c|c|c|c|c|}
\hline Group & A-value ${ }^{a}$ & $\mathrm{~L}^{b}$ & B1 ${ }^{b}$ & $\mathrm{~B}^{b}$ & $B 1^{\prime c}$ & $\mathrm{Pol}^{d}$ & A-value ${ }^{e}$ & $L^{e}$ & $\mathrm{B5}^{e}$ & $B 1^{\prime}{ }^{e}$ & $\mathrm{Pol}^{e}$ & B1 $^{\prime}{ }^{e}-$ A-value ${ }^{e}$ \\
\hline $\mathrm{H}$ & 0 & 2.23 & 1.09 & 1.09 & 1.09 & 0.77 & 0 & 0 & 0 & 0 & 0 & 0 \\
\hline $\mathrm{F}$ & 0.335 & 2.79 & 1.47 & 1.47 & 1.47 & 0.81 & 0.069792 & 0.090762 & 0.055637 & 0.186275 & 0.001922 & 0.116483 \\
\hline $\mathrm{Cl}$ & 0.585 & 3.42 & 1.75 & 1.75 & 1.75 & 2.53 & 0.121875 & 0.192869 & 0.096633 & 0.323529 & 0.091298 & 0.201654 \\
\hline $\mathrm{Br}$ & 0.575 & 3.66 & 1.85 & 1.85 & 1.85 & 3.48 & 0.119792 & 0.231767 & 0.111274 & 0.372549 & 0.140871 & 0.252757 \\
\hline 1 & 0.54 & 3.98 & 1.98 & 1.98 & 1.98 & 5.24 & 0.1125 & 0.28363 & 0.130307 & 0.436275 & 0.231999 & 0.323775 \\
\hline $\mathrm{OH}$ & 0.82 & 2.88 & 1.05 & 2.02 & 1.52 & 1.42 & 0.170833 & 0.105348 & 0.136164 & 0.210784 & 0.033965 & 0.039951 \\
\hline OMe & 0.6275 & 3.98 & 1.4 & 3.18 & 1.52 & 3.12 & 0.130729 & 0.28363 & 0.306003 & 0.210784 & 0.12197 & 0.080055 \\
\hline OtBu & 0.75 & 5.02 & 1.44 & 4.45 & 1.52 & 8.43 & 0.15625 & 0.452188 & 0.491947 & 0.210784 & 0.397125 & 0.054534 \\
\hline $\mathrm{OPh}$ & 0.65 & 5.84 & 1.52 & 5.99 & 1.52 & 11.06 & 0.135417 & 0.585089 & 0.717423 & 0.210784 & 0.533769 & 0.075368 \\
\hline OPhCl-p & 0.65 & 5.86 & 1.52 & 7.24 & 1.52 & 13.22 & 0.135417 & 0.588331 & 0.900439 & 0.210784 & 0.645968 & 0.075368 \\
\hline OPhOMe-p & 0.7 & 6.08 & 1.52 & 7.92 & 1.52 & 13.78 & 0.145833 & 0.623987 & 1 & 0.210784 & 0.674919 & 0.064951 \\
\hline $\mathrm{OCHO}$ & 0.435 & 3.93 & 1.46 & 3.76 & 1.52 & 3.34 & 0.090625 & 0.275527 & 0.390922 & 0.210784 & 0.133335 & 0.120159 \\
\hline ocOMe & 0.7625 & 4.93 & 1.24 & 3.73 & 1.52 & 5.05 & 0.158854 & 0.437601 & 0.38653 & 0.210784 & 0.22215 & 0.05193 \\
\hline $\mathrm{OCOCF}_{3}$ & 0.62 & 5.26 & 1.52 & 4.08 & 1.52 & 5.33 & 0.129167 & 0.491086 & 0.437775 & 0.210784 & 0.236794 & 0.081618 \\
\hline OCOPh & 0.5 & 8.1 & 1.52 & 5.14 & 1.52 & 13.28 & 0.104167 & 0.951378 & 0.592972 & 0.210784 & 0.648965 & 0.106618 \\
\hline OCONHPh & 0.77 & 8.25 & 1.47 & 7.05 & 1.52 & 14.93 & 0.160417 & 0.975689 & 0.872621 & 0.210784 & 0.73427 & 0.050368 \\
\hline $\mathrm{OSiMe}_{3}$ & 0.74 & 5.65 & 1.46 & 4.85 & 1.52 & 10.02 & 0.154167 & 0.554295 & 0.550512 & 0.210784 & 0.479638 & 0.056618 \\
\hline SH & 1.21 & 3.54 & 1.12 & 2.42 & 1.8 & 3.61 & 0.252083 & 0.212318 & 0.194729 & 0.348039 & 0.147436 & 0.095956 \\
\hline SMe & 1.04 & 4.11 & 1.53 & 3.51 & 1.8 & 5.41 & 0.216667 & 0.3047 & 0.354319 & 0.348039 & 0.240517 & 0.131373 \\
\hline $\mathrm{SPh}$ & 1.17 & 5.55 & 1.8 & 6.68 & 1.8 & 13.70 & 0.24375 & 0.538088 & 0.818448 & 0.348039 & 0.67056 & 0.104289 \\
\hline SOMe & 1.2 & 3.96 & 1.52 & 3.51 & 1.8 & 6.04 & 0.25 & 0.280389 & 0.354319 & 0.348039 & 0.273309 & 0.098039 \\
\hline SCN & 1.23 & 3.57 & 1.79 & 4.41 & 1.8 & 5.49 & 0.25625 & 0.21718 & 0.486091 & 0.348039 & 0.245005 & 0.091789 \\
\hline SePh & 1.1 & 5.27 & 1.88 & 6.83 & 1.9 & 14.67 & 0.229167 & 0.492707 & 0.84041 & 0.397059 & 0.72116 & 0.167892 \\
\hline $\mathrm{NH}_{2}$ & 1.35 & 2.96 & 1.05 & 2.06 & 1.55 & 2.10 & 0.28125 & 0.118314 & 0.14202 & 0.22549 & 0.069211 & -0.05576 \\
\hline $\mathrm{NHMe}$ & 1.29 & 4.1 & 1.06 & 3.25 & 1.55 & 3.80 & 0.26875 & 0.303079 & 0.316252 & 0.22549 & 0.157142 & -0.04326 \\
\hline $\mathrm{NMe}_{2}$ & 1.53 & 4.06 & 1.41 & 3.22 & 1.55 & 5.61 & 0.31875 & 0.296596 & 0.311859 & 0.22549 & 0.251009 & -0.09326 \\
\hline NHCOPh & 1.6 & 8.4 & 1.75 & 4.7 & 1.75 & 13.98 & 0.333333 & 1 & 0.528551 & 0.323529 & 0.685055 & -0.0098 \\
\hline $\mathrm{PMe}_{2}$ & 1.55 & 4.19 & 1.54 & 3.54 & 1.8 & 8.12 & 0.322917 & 0.317666 & 0.358712 & 0.348039 & 0.381213 & 0.025123 \\
\hline $\mathrm{PCl}_{2}$ & 1.95 & 3.74 & 1.8 & 3.8 & 1.8 & 8.21 & 0.40625 & 0.244733 & 0.396779 & 0.348039 & 0.385933 & -0.05821 \\
\hline $\mathrm{P}(\mathrm{OMe})_{2}$ & 1.7 & 4.88 & 1.7 & 4.62 & 1.8 & 8.98 & 0.354167 & 0.429498 & 0.516837 & 0.348039 & 0.425886 & -0.00613 \\
\hline $\mathrm{CHO}$ & 0.6966666667 & 3.66 & 1.6 & 2.55 & 1.7 & 2.61 & 0.145139 & 0.231767 & 0.213763 & 0.29902 & 0.095505 & 0.153881 \\
\hline COMe & 1.25 & 4.32 & 1.7 & 3.19 & 1.7 & 4.45 & 0.260417 & 0.338736 & 0.307467 & 0.29902 & 0.190688 & 0.038603 \\
\hline $\mathrm{CO} 2 \mathrm{H}$ & 1.4 & 4.01 & 1.59 & 2.79 & 1.7 & 3.34 & 0.291667 & 0.288493 & 0.248902 & 0.29902 & 0.133335 & 0.007353 \\
\hline $\mathrm{CO} 2 \mathrm{Me}$ & 1.25 & 5.07 & 1.75 & 3.54 & 1.75 & 5.04 & 0.260417 & 0.460292 & 0.358712 & 0.323529 & 0.22161 & 0.063113 \\
\hline $\mathrm{CO} 2 \mathrm{Et}$ & 1.15 & 5.85 & 1.75 & 4.57 & 1.75 & 6.87 & 0.239583 & 0.58671 & 0.509517 & 0.323529 & 0.316248 & 0.083946 \\
\hline COF & 1.55 & 3.74 & 1.58 & 2.74 & 1.7 & 2.69 & 0.322917 & 0.244733 & 0.241581 & 0.29902 & 0.099411 & -0.0239 \\
\hline $\mathrm{COCl}$ & 1.3 & 3.84 & 1.59 & 3.41 & 1.7 & 4.71 & 0.270833 & 0.26094 & 0.339678 & 0.29902 & 0.204596 & 0.028186 \\
\hline $\mathrm{CN}$ & 0.2 & 4.18 & 1.7 & 1.7 & 1.7 & 2.48 & 0.041667 & 0.316045 & 0.089312 & 0.29902 & 0.088988 & 0.257353 \\
\hline $\mathrm{CCH}$ & 0.465 & 4.83 & 1.7 & 1.7 & 1.7 & 3.38 & 0.096875 & 0.421394 & 0.089312 & 0.29902 & 0.135591 & 0.202145 \\
\hline $\mathrm{CH}=\mathrm{CH} 2$ & 1.585 & 4.35 & 1.7 & 3.19 & 1.7 & 4.03 & 0.330208 & 0.343598 & 0.307467 & 0.29902 & 0.169204 & -0.03119 \\
\hline $\mathrm{CH}=\mathrm{C}=\mathrm{CH} 2$ & 1.53 & 4.52 & 1.56 & 3.97 & 1.7 & 5.93 & 0.31875 & 0.371151 & 0.421669 & 0.29902 & 0.267753 & -0.01973 \\
\hline
\end{tabular}




\begin{tabular}{|c|c|c|c|c|c|c|c|c|c|c|c|c|}
\hline $\mathrm{Me}$ & 1.74 & 3.19 & 1.7 & 2.11 & 1.7 & 2.45 & 0.3625 & 0.155592 & 0.149341 & 0.29902 & 0.086974 & -0.06348 \\
\hline Et & 1.79 & 4.22 & 1.7 & 3.25 & 1.7 & 4.19 & 0.372917 & 0.322528 & 0.316252 & 0.29902 & 0.177487 & -0.0739 \\
\hline $\mathrm{iPr}$ & 2.21 & 4.18 & 2 & 3.26 & 2 & 5.97 & 0.460417 & 0.316045 & 0.317716 & 0.446078 & 0.269661 & -0.01434 \\
\hline $\mathrm{tBu}$ & 4.8 & 4.16 & 2.85 & 3.26 & 2.85 & 7.76 & 1 & 0.312804 & 0.317716 & 0.862745 & 0.362464 & -0.13725 \\
\hline $\mathrm{CH} 2 \mathrm{Br}$ & 1.79 & 3.93 & 1.7 & 3.68 & 1.7 & 5.35 & 0.372917 & 0.275527 & 0.379209 & 0.29902 & 0.237548 & -0.0739 \\
\hline $\mathrm{CH}_{2} \mathrm{OH}$ & 1.76 & 3.94 & 1.7 & 2.89 & 1.7 & 3.12 & 0.366667 & 0.277147 & 0.263543 & 0.29902 & 0.12197 & -0.06765 \\
\hline $\mathrm{CH}_{2} \mathrm{OCH}$ & 1.72 & 4.23 & 1.7 & 3.59 & 1.7 & 4.45 & 0.358333 & 0.324149 & 0.366032 & 0.29902 & 0.190697 & -0.05931 \\
\hline $\mathrm{CH}_{2} \mathrm{CN}$ & 1.77 & 3.93 & 1.7 & 4.02 & 1.7 & 4.30 & 0.36875 & 0.275527 & 0.42899 & 0.29902 & 0.18333 & -0.06973 \\
\hline $\mathrm{CH}_{2} \mathrm{SiMe}_{3}$ & 1.65 & 5.75 & 1.7 & 5.09 & 1.7 & 11.44 & 0.34375 & 0.570502 & 0.585652 & 0.29902 & 0.553357 & -0.04473 \\
\hline $\mathrm{CH}_{2} \mathrm{SnMe}_{3}$ & 1.79 & 6.07 & 1.7 & 5.5 & 1.7 & 13.59 & 0.372917 & 0.622366 & 0.645681 & 0.29902 & 0.664923 & -0.0739 \\
\hline $\mathrm{CF}_{3}$ & 2.45 & 3.42 & 2.1 & 2.72 & 2.1 & 2.72 & 0.510417 & 0.192869 & 0.238653 & 0.495098 & 0.101456 & -0.01532 \\
\hline $\mathrm{Ph}$ & 2.8 & 6.44 & 1.7 & 3.23 & 1.7 & 10.24 & 0.583333 & 0.682334 & 0.313324 & 0.29902 & 0.491037 & -0.28431 \\
\hline $\mathrm{CH}_{2} \mathrm{Ph}$ & 1.68 & 4.67 & 1.7 & 6.16 & 1.7 & 12.18 & 0.35 & 0.395462 & 0.742313 & 0.29902 & 0.591769 & -0.05098 \\
\hline $\mathrm{C}_{6} \mathrm{H}_{11}$ & 2.2 & 6.26 & 2 & 3.57 & 2 & 10.40 & 0.458333 & 0.65316 & 0.363104 & 0.446078 & 0.499676 & -0.01225 \\
\hline $\mathrm{SiH}_{3}$ & 1.485 & 3.98 & 2.1 & 2.48 & 2.1 & 4.55 & 0.309375 & 0.28363 & 0.203514 & 0.495098 & 0.195874 & 0.185723 \\
\hline $\mathrm{SiMe}_{3}$ & 2.5 & 4.68 & 3.01 & 3.57 & 3.01 & 9.72 & 0.520833 & 0.397083 & 0.363104 & 0.941176 & 0.464112 & 0.420343 \\
\hline $\mathrm{SiCl}_{3}$ & 0.61 & 4.3 & 2.71 & 3.67 & 2.71 & 9.60 & 0.127083 & 0.335494 & 0.377745 & 0.794118 & 0.458036 & 0.667034 \\
\hline $\mathrm{GeMe}_{3}$ & 2.1 & 4.75 & 3.04 & 3.61 & 3.04 & 10.27 & 0.4375 & 0.408428 & 0.36896 & 0.955882 & 0.492706 & 0.518382 \\
\hline $\mathrm{SnMe}_{3}$ & 1 & 4.99 & 3.13 & 3.78 & 3.13 & 11.80 & 0.208333 & 0.447326 & 0.393851 & 1 & 0.57228 & 0.791667 \\
\hline $\mathrm{SnMe}_{2} \mathrm{Ph}$ & 1.08 & 6.69 & 3.1 & 6.82 & 3.1 & 20.05 & 0.225 & 0.722853 & 0.838946 & 0.985294 & 1 & 0.760294 \\
\hline
\end{tabular}

${ }^{a} \mathrm{~A}$-values were taken as average values. ${ }^{30}$

${ }^{b}$ Computed Sterimol parameters from R-H geometries optimized at MP2/aug-cc-pVTZ. ${ }^{31}$

${ }^{c}$ B1' is the B1 Sterimol parameter, corrected such that values of B1 that were lower than the vdW radii of the first atom were corrected to equal the vdW radius. ${ }^{32}$

${ }^{d}$ Computed isotropic polarizability $\left(10^{-24} \mathrm{esu}\right)$.

${ }^{e}$ These are normalized values, computed as $\left(x-x_{\min }\right) /\left(x_{\max }-x_{\min }\right)$. 
6. Systems 2,3 , and 4

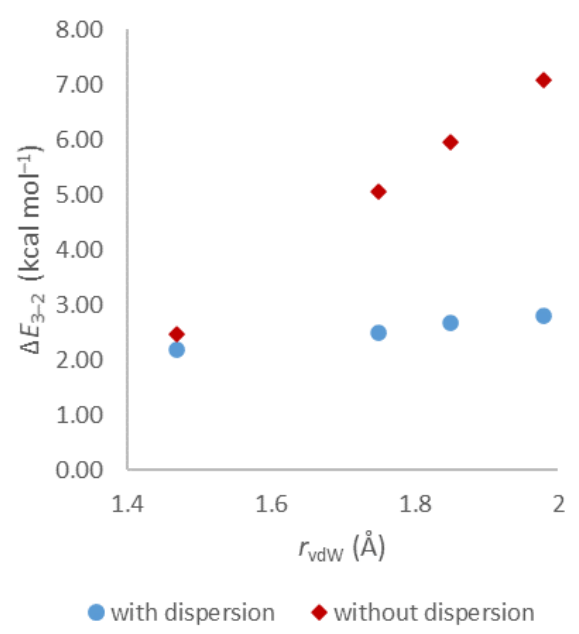

Figure S9. Plot of the difference in electronic energies, $\Delta E_{3-2}$, against the halogens' vdW radii. Computed at the B3LYP-D3BJ/def2-TZVPP and B3LYP/def2-TZVPP//B3LYP-D3BJ/def2-TZVPP levels of theory.

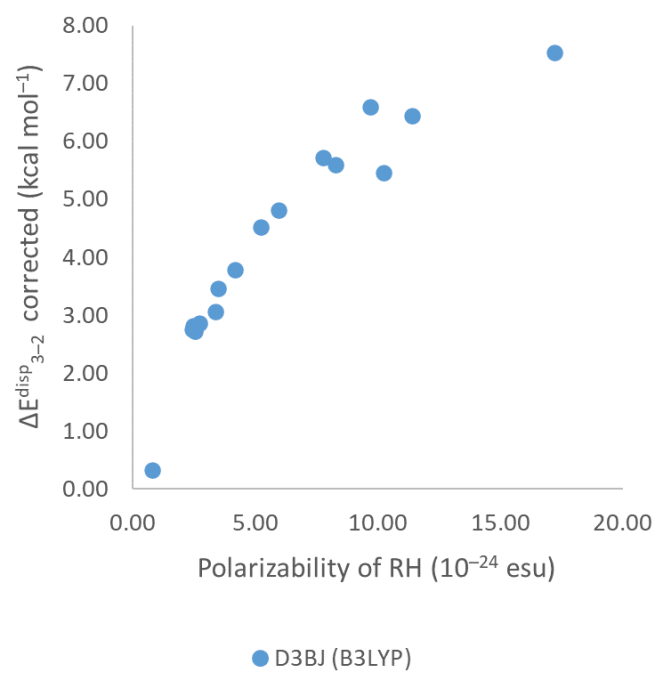

Figure S10. Plot of the LD energy from the D3BJ correction in the 3-2 system, against the computed polarizabilities of the RH molecules (formed from group R with an added hydrogen). 
Table S12. Single point energies $\left(\mathrm{kcal} \mathrm{mol}^{-1}\right)$ for substituted cyclohexylacetylene, from geometries optimized at MP2/aug-cc-pVTZ.

\begin{tabular}{|c|c|c|c|c|c|}
\hline & HF/aug-cc-pVTZ & MP2/aug-cc-pVTZ & B3LYP/def2-TZVPP & $\begin{array}{c}\text { B3LYP-D3(BJ)/def2- } \\
\text { TZVPP }\end{array}$ & $\begin{array}{c}\text { DLPNO-CCSD(T)/aug-cC- } \\
\text { pVQZ }\end{array}$ \\
\hline $4 \mathrm{H}_{\mathrm{eq}}$ & -309.983503270 & -311.338329600 & -312.14110233 & -312.17350044 & -311.533556644 \\
\hline $4 \mathrm{H}_{\mathrm{ax}}$ & -309.981162355 & -311.338662400 & -312.13926391 & -312.17296297 & -311.533151278 \\
\hline $4 \mathrm{Me}_{\text {eq }}$ & -349.040177104 & -350.570818587 & -351.48002652 & -351.51610355 & -350.792437348 \\
\hline $4 \mathrm{Me}_{\mathrm{ax}}$ & -349.037614123 & -350.571280145 & -351.47805195 & -351.51563503 & -350.792118162 \\
\hline $4 t B u_{e q}$ & -466.182879073 & -468.253785380 & -469.46595372 & -469.51937083 & -468.553404534 \\
\hline $4 \mathrm{tBu} \mathrm{uax}$ & -466.179898454 & -468.254761278 & -469.46357972 & -469.51915037 & -468.553354656 \\
\hline 4 SiMeзеq $_{\text {- }}$ & -717.299468316 & -719.318525655 & -720.91938740 & -720.97410648 & -719.626246887 \\
\hline $4 \mathrm{SiMe}_{3 \mathrm{ax}}$ & -717.296650330 & -719.319492904 & -720.91717910 & -720.97395986 & -719.626157762 \\
\hline $4 C y_{\text {eqeq }}$ & -543.115157515 & -545.511846891 & -546.91457876 & -546.97780399 & -545.855815060 \\
\hline $4 C y_{\text {axeq }}$ & -543.112079922 & -545.512917483 & -546.91211821 & -546.97764756 & -545.855854968 \\
\hline $4 C У_{\text {ахах }}$ & -543.107492358 & -545.515317077 & -546.90872711 & -546.97832670 & -545.856706251 \\
\hline
\end{tabular}

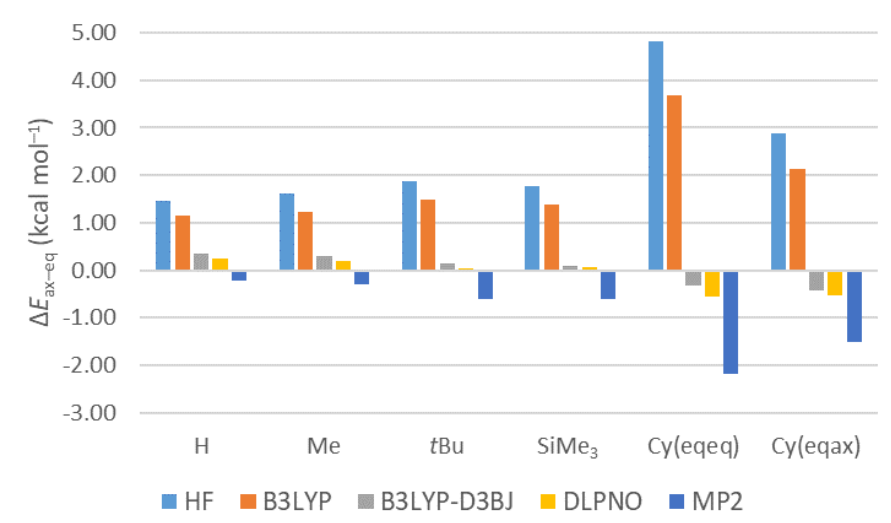

Figure S11. $\Delta E_{\text {ax-eq }}$ values for 4 with different $\mathrm{R}$ groups. For $\mathrm{R}=\mathrm{Cy}$ the electronic energy differences depicted are $\Delta E_{\text {axax-eqeq }}$ and $\Delta E_{\text {axax-eqax. }}$ Levels of theory used are HF/aug-cc-pVTZ//MP2/aug-cc-pVTZ, B3LYP/def2TZVPP//MP2/aug-cc-pVTZ, $\quad$ B3LYP-D3BJ/def2-TZVPP//MP2/aug-cc-pVTZ, $\quad$ DLPNO-CCSD(T)/aug-ccpVQZ//MP2/aug-cc-pVTZ, MP2/aug-cc-pVTZ:

We computed relaxed energy scans for the three conformers of 4Cy against the angle defined by atoms 1-2-3-4 (Fig. S12, see Fig. 8 in the main text for numbering). Qualitatively the energy scan graphs look similar for B3LYP-D3BJ and MP2. However, the B3LYP graphs without LD correction look different, showing higher preference for the structures with an angle of $180^{\circ}$. The difference caused by the LD correction is smallest for $\mathbf{4 C} \mathbf{y}_{\text {eqeq, }}$ a little larger for $\mathbf{4} \mathbf{C}_{\text {eqax }}$, and the largest for $\mathbf{4 C} \mathbf{y}_{\text {axax }}$, showing that the LD interactions become more significant when the two rings are above one another, as expected. These interactions stabilize the conformer with an angle around $19.4^{\circ}$ for $\mathbf{4 C} y_{\text {axax }}$, a conformer that is not observed without the LD correction. Unlike $\mathbf{4} \mathbf{C y}_{\text {axax }}$, the conformers $\mathbf{4} \mathbf{C y}_{\text {eqeq }}$ and $\mathbf{4 C} \mathbf{y}_{\text {eqax }}$ show quite 
a shallow potential energy surface (scanning an energy range of 0.07 and $0.33 \mathrm{kcal} \mathrm{mol}^{-1}$, respectively, from MP2, and 0.1 and $0.2 \mathrm{kcal} \mathrm{mol}^{-1}$, respectively, from B3LYP-D3BJ).

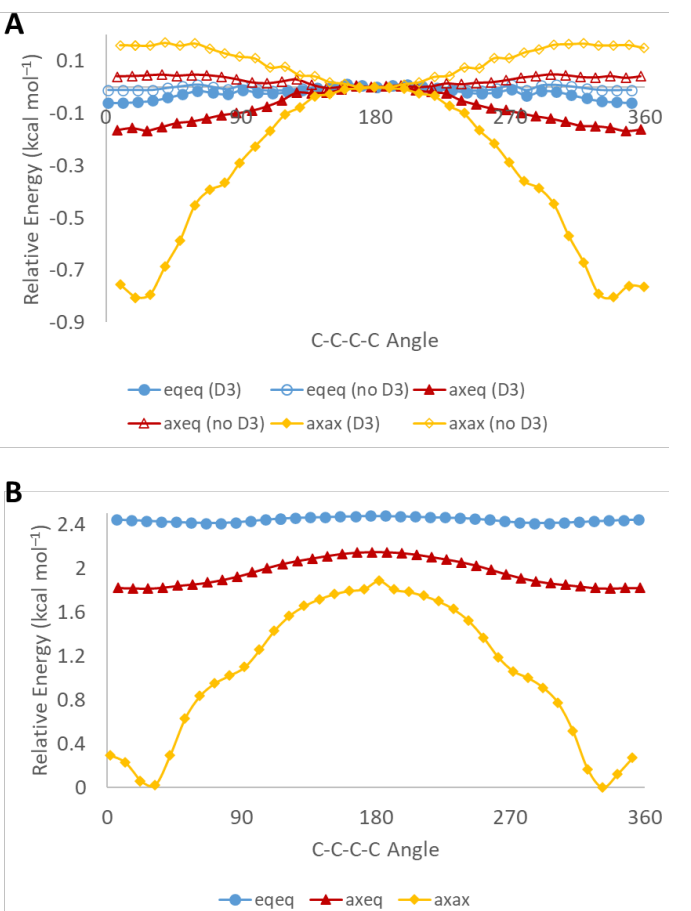

Figure S12. Relaxed electronic energy scans for 4Cy computed at B3LYP/def2-TZVPP (A) and MP2/aug-cc-pVDZ (B). For B3LYP all graphs are made to intersect zero at $180^{\circ}$.

Table S13. Relative computed electronic energy $(\Delta E)$, zero-point vibrational corrected energy ( $E+Z P V E)$, enthalpy $\left(\Delta H_{0}\right)$ and free energy $(\Delta G)$ in $\mathrm{kcal} \mathrm{mol}^{-1}$ of the three conformers of $4 \mathrm{Cy}$. Computed at the B3LYPD3BJ/def2-TZVPP level of theory.

\begin{tabular}{|c|c|c|c|c|c|c|}
\hline & $\Delta \mathrm{E}$ & $\Delta E+\mathrm{ZPVE}$ & $\Delta \mathrm{H}$ & $\Delta G(4 \mathrm{~K})$ & $\Delta G(193 \mathrm{~K})$ & $\Delta G(298 \mathrm{~K})$ \\
\hline $\begin{array}{c}1 \mathrm{e}_{\text {eqeq }}\left(\alpha_{1-2-3-4}=\right. \\
12.8)\end{array}$ & 0.6 & 0.2 & 0.4 & 0.2 & 0.3 & 0.6 \\
\hline $\begin{array}{c}1 \mathrm{e}_{\text {eqeq }}\left(\alpha_{1-2-3-4}=\right. \\
108.9)\end{array}$ & 0.6 & 0.2 & 0.4 & 0.2 & 0.0 & 0.0 \\
\hline $1 \mathrm{e}_{\text {eqax }}$ & 0.6 & 0.4 & 0.5 & 0.4 & 0.8 & 1.3 \\
\hline $1 \mathrm{e}_{\text {axax }}$ & 0.0 & 0.0 & 0.0 & 0.0 & 0.8 & 1.4 \\
\hline
\end{tabular}

*Computed without applying the quasi-harmonic approximation. 


\section{Cartesian Coordinates}

\section{Cy (MP2/aug-cc-pVDZ)}

Imaginary frequencies- 0

Electronic Energy (SCF) $=-234.230885009$ a.u Electronic Energy (MP2) $=-235.1216495879$ a.u ZPV corrected Energy = -234.951201 a.u. Enthalpy $=-234.944524$ a.u.

Free Energy $=-234.978287$ a.u.

$\begin{array}{llll}\text { C } & 1.266145 & 0.731009 & 0.236915\end{array}$

C $\quad 0.000000 \quad 1.462019-0.236915$

H $\quad 0.000000 \quad 1.507003 \quad-1.342457$

H $\quad 0.000000 \quad 2.503970 \quad 0.126609$

C $1.266145 \quad-0.731009-0.236915$

H $\quad 2.168502 \quad-1.251985 \quad 0.126609$

H $\quad 1.305103 \quad-0.753502-1.342457$

C $\quad-0.000000 \quad-1.462019 \quad 0.236915$

H $\quad-0.000000-2.503970-0.126609$

H $\quad-0.000000-1.507003 \quad 1.342457$

C $\quad-1.266145 \quad 0.731009 \quad 0.236915$

$\begin{array}{llll}H & -1.305103 & 0.753502 & 1.342457\end{array}$

H $\quad-2.168502 \quad 1.251985-0.126609$

C $-1.266145 \quad-0.731009-0.236915$

H $\quad-2.168502 \quad-1.251985 \quad 0.126609$

$\begin{array}{llll}\text { H } & -1.305103 & -0.753502 & -1.342457\end{array}$

H $\quad 2.168502 \quad 1.251985-0.126609$

H $\quad 1.305103 \quad 0.753502 \quad 1.342457$

\section{Cy (MP2/aug-cc-pVTZ)}

Imaginary frequencies- 0

Electronic Energy (SCF) $=-234.287164989$ a.u Electronic Energy (MP2) $=-235.3465173503$ a.u ZPV corrected Energy = -235.174858 a.u.

Enthalpy $=-235.168216$ a.u.

Free Energy = -235.203607 a.u.

$\begin{array}{llll}\text { C } & 0.791415 & -1.218281 & 0.234901\end{array}$

C $1.450771 \quad 0.076245-0.234902$

H $1.4945390 .078545 \quad-1.328975$

H $\quad 2.480544 \quad 0.1303640 .124142$

C $-0.659355-1.294526-0.234901$

H $\quad-1.127372-2.213396 \quad 0.124141$

H $\quad-0.679248-1.333580-1.328974$

$\begin{array}{llll}\text { C } & -1.450771 & -0.076245 & 0.234902\end{array}$

H $\quad-2.480544-0.130364-0.124142$

H $\quad-1.494539-0.078545 \quad 1.328975$

$\begin{array}{llll}\text { C } & 0.659355 & 1.294526 & 0.234901\end{array}$

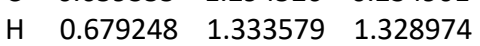

H $\quad 1.127372 \quad 2.213396-0.124141$

C $\quad-0.791415 \quad 1.218281-0.234901$

H $\quad-1.3531692 .083032 \quad 0.124141$

H $\quad-0.815291 \quad 1.255035-1.328974$

H $\quad 1.353169-2.083032-0.124141$

H $\quad 0.815291 \quad-1.255035 \quad 1.328974$

\section{Cy (MP2/aug-cc-pVQZ)}

Imaginary frequencies- 0

Electronic Energy (SCF) = -234.300993727 a.u
Electronic Energy (MP2) $=-235.4145354056$ a.u ZPV corrected Energy = -235.242806 a.u. Enthalpy $=-235.236166$ a.u.

Free Energy $=-235.271547$ a.u.

C $-1.256091-0.725202-0.234638$

C $\quad-1.256080 \quad 0.7252180 .234620$

H $\quad-1.293450 \quad 0.746847 \quad 1.327278$

H $\quad-2.148254 \quad 1.240320-0.122931$

C $\quad-0.000014 \quad-1.450374 \quad 0.234745$

$\begin{array}{llll}\text { H } & -0.000029 & -2.480619 & -0.122678\end{array}$

H $\quad-0.000020-1.493425 \quad 1.327408$

C $1.256080-0.725218-0.234620$

H $\quad 2.148254 \quad-1.240320 \quad 0.122931$

H $\quad 1.293450 \quad-0.746847 \quad-1.327278$

C $\quad 0.000014 \quad 1.450374 \quad-0.234745$

H $\quad 0.000020 \quad 1.493425-1.327408$

$\begin{array}{llll}\text { H } & 0.000029 & 2.480619 & 0.122678\end{array}$

C $\quad 1.256091 \quad 0.7252020 .234638$

H $\quad 2.148292 \quad 1.240289-0.122868$

H $\quad \begin{array}{llll}\text { H.293421 } & 0.746818 & 1.327302\end{array}$

H $\quad-2.148292 \quad-1.2402890 .122868$

H $\quad-1.293421 \quad-0.746818-1.327302$

\section{Cy (MP2/QZVP)}

Imaginary frequencies- 0

Electronic Energy (SCF) $=-234.302930270$ a.u Electronic Energy (MP2) $=-235.4079644561$ a.u ZPV corrected Energy = -235.236146 a.u.

Enthalpy $=-235.229506$ a.u.

Free Energy $=-235.264888$ a.u.

$\begin{array}{llll}\text { C } & 1.261819 & 0.714198 & 0.234635\end{array}$

C $0.012396 \quad 1.449865 \quad-0.234634$

H $\quad 0.012766 \quad 1.493153 \quad-1.326926$

$\begin{array}{llll}H & 0.021201 & 2.479742 & 0.122637\end{array}$

C $1.249423 \quad-0.735668-0.234635$

H $\quad 2.136919 \quad-1.258233 \quad 0.122638$

H $\quad \begin{array}{llll}\text { H } & 1.286727 & -0.757633 & -1.326928\end{array}$

$\begin{array}{llll}\text { C } & -0.012396 & -1.449865 & 0.234634\end{array}$

H $\quad-0.021201-2.479742-0.122637$

H $\quad-0.012766-1.493153 \quad 1.326926$

C $\quad-1.249423 \quad 0.7356680 .234635$

$\begin{array}{llll}\text { H } & -1.286727 & 0.757633 & 1.326928\end{array}$

H $\quad-2.136919 \quad 1.258233-0.122638$

$\begin{array}{llll}\text { C } & -1.261819 & -0.714198 & -0.234635\end{array}$

H $-2.158121 \quad-1.2215110 .122638$

H $\quad-1.299493 \quad-0.735522 \quad-1.326928$

H $\quad 2.158121 \quad 1.221511-0.122638$

H $\quad 1.299493 \quad 0.735522 \quad 1.326928$

\section{$1 F_{\text {eq }}$ (MP2/aug-cc-pVTZ)}

Imaginary frequencies- 0

Electronic Energy (SCF) $=-333.185502290$ a.u Electronic Energy (MP2) $=-334.4898404308$ a.u ZPV corrected Energy = -334.325798 a.u.

Enthalpy $=-334.318425$ a.u. 
Free Energy $=-334.355924$ a.u.

$\begin{array}{llll}C & 0.581331 & 0.895027 & 0.000000\end{array}$

$\begin{array}{llll}\text { C } & -0.089877 & 0.378327 & 1.253065\end{array}$

H $\quad-1.1173390 .751868 \quad 1.267943$

$\begin{array}{llll}\text { H } & 0.420326 & 0.775380 & 2.131515\end{array}$

C $\quad-0.089877 \quad 0.378327 \quad-1.253065$

H $\quad 0.420326 \quad 0.775380-2.131515$

H $\quad-1.1173390 .751868-1.267943$

C $-0.089877-1.150391-1.256370$

H $\quad-0.590624-1.519035-2.152658$

H $\quad 0.941749-1.513383-1.299173$

C $\quad-0.089877-1.150391 \quad 1.256370$

H $0.941749-1.513383 \quad 1.299173$

H $\quad-0.590624-1.519035 \quad 2.152658$

C $\quad-0.762129-1.698096-0.000000$

H $\quad-0.732891 \quad-2.789141-0.000000$

H $-1.816608-1.405650-0.000000$

H $\quad 1.640610 \quad 0.6195190 .000000$

$\begin{array}{llll}F & 0.538057 & 2.296533 & 0.000000\end{array}$

\section{$1 \mathrm{~F}_{\mathrm{ax}}$ (MP2/aug-cc-pVTZ)}

Imaginary frequencies- 0

Electronic Energy (SCF) = -333.185090341 a.u Electronic Energy (MP2) $=-334.4896487106$ a.u ZPV corrected Energy $=-334.325532$ a.u. Enthalpy $=-334.318178$ a.u.

Free Energy = -334.355615 a.u.

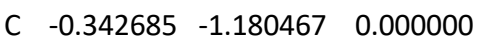

$\begin{array}{llll}\text { C } & 0.283325 & -0.618497 & 1.261037\end{array}$

H $1.310959-0.990043 \quad 1.317056$

H $\quad-0.252079-1.008831 \quad 2.127767$

$\begin{array}{llll}\text { C } & 0.283325 & -0.618497 & -1.261037\end{array}$

H $\quad-0.252079-1.008831-2.127767$

H $\quad 1.310959-0.990043-1.317056$

C $0.283325 \quad 0.908067 \quad-1.254788$

H $\quad 0.779183 \quad 1.280826-2.152474$

H $\quad-0.748564 \quad 1.265253-1.285533$

C $\quad 0.283325 \quad 0.908067 \quad 1.254788$

H $\quad-0.748564 \quad 1.265253 \quad 1.285533$

H $\quad 0.779183 \quad 1.280826 \quad 2.152474$

C $0.965979 \quad 1.446318-0.000000$

H $\quad 0.952149 \quad 2.537633-0.000000$

H $\quad 2.017610 \quad 1.140219-0.000000$

H $\quad-0.303784 \quad-2.271415 \quad 0.000000$

$\begin{array}{llll}\text { F } & -1.709393 & -0.841199 & 0.000000\end{array}$

\section{Cleq (MP2/aug-cc-pVTZ)}

Imaginary frequencies- 0

Electronic Energy (SCF) $=-693.230531574$ a.u Electronic Energy (MP2) $=-694.4764068766$ a.u ZPV corrected Energy $=-694.313845$ a.u.

Enthalpy $=-694.306161$ a.u.

Free Energy $=-694.344848$ a.u.

C $\quad 0.508956 \quad 0.503980 \quad 0.000000$

C $\quad-0.164750 \quad-0.015408 \quad 1.256420$

$\begin{array}{llll}\text { H } & -1.192515 & 0.357852 & 1.276086\end{array}$

$\begin{array}{llll}H & 0.343084 & 0.377565 & 2.137968\end{array}$

$\begin{array}{lrrr}\text { C } & -0.164750 & -0.015408 & -1.256420\end{array}$
$\begin{array}{llll}H & 0.343084 & 0.377565 & -2.137968\end{array}$

H $\quad-1.192515 \quad 0.357852 \quad-1.276086$

C $-0.164750-1.544342-1.255478$

H $\quad-0.665923-1.911690-2.152559$

H $0.867190 \quad-1.906676-1.297903$

C $\quad-0.164750 \quad-1.544342 \quad 1.255478$

H $\quad 0.867190 \quad-1.906676 \quad 1.297903$

H $\quad-0.665923 \quad-1.911690 \quad 2.152559$

C $-0.838608-2.090610-0.000000$

H $-0.812906-3.181731-0.000000$

H $\quad-1.892244-1.794790-0.000000$

H 1.5651130 .2288110 .000000

Cl $0.492252 \quad 2.302965 \quad 0.000000$

\section{Cl ${ }_{\text {ax }}$ (MP2/aug-cc-pVTZ)}

Imaginary frequencies- 0

Electronic Energy (SCF) = -693.228492587 a.u Electronic Energy (MP2) $=-694.4760907080$ a.u ZPV corrected Energy = -694.313361 a.u.

Enthalpy $=-694.305783$ a.u.

Free Energy = -694.344187 a.u.

C $\quad-0.021449-1.082936 \quad-0.000000$

C $0.605196 \quad-0.507235 \quad 1.260782$

H $1.638525 \quad-0.869673 \quad 1.296722$

H $\quad 0.090325 \quad-0.899474 \quad 2.138476$

C $0.605196 \quad-0.507235-1.260782$

H $\quad 0.090325 \quad-0.899474 \quad-2.138476$

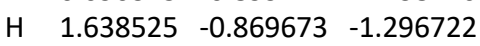

C $\quad 0.605196 \quad 1.018150 \quad-1.257315$

H $\quad 1.111174 \quad 1.385138-2.151900$

H $\quad-0.425423 \quad 1.377750 \quad-1.300440$

C $0.605196 \quad 1.018150 \quad 1.257315$

H $\quad-0.425423 \quad 1.377750 \quad 1.300440$

H $\quad \begin{array}{llll}1.111174 & 1.385138 & 2.151900\end{array}$

C $\quad 1.281543 \quad 1.559165 \quad 0.000000$

H $\quad 1.261412 \quad 2.650400 \quad 0.000000$

H $\quad 2.335117 \quad 1.259746 \quad 0.000000$

H $\quad 0.046644-2.169391-0.000000$

Cl $-1.797508-0.748035 \quad-0.000000$

\section{$1 \mathrm{Br}_{\text {eq }}$ (MP2/aug-cc-pVTZ)}

Imaginary frequencies- 0

Electronic Energy (SCF) $=-2806.17260741$ a.u Electronic Energy (MP2) $=-2807.4641259218 \mathrm{a} . \mathrm{u}$ ZPV corrected Energy $=-2807.302162$ a.u. Enthalpy $=-2807.294280$ a.u.

Free Energy $=-2807.334238$ a.u.

$\begin{array}{llll}\text { C } & -0.397950 & 0.065289 & -0.000000\end{array}$

C $\quad 0.274675 \quad 0.581274 \quad 1.257484$

$\begin{array}{llll}\text { H } & 1.302634 & 0.208497 & 1.280122\end{array}$

H $\quad-0.233778 \quad 0.190386 \quad 2.139509$

C $0.274675 \quad 0.581274-1.257484$

H $\quad-0.233778 \quad 0.190386 \quad-2.139509$

H $\quad 1.302634 \quad 0.208497 \quad-1.280122$

C $0.274675 \quad 2.110556-1.255382$

H $\quad 0.775470 \quad 2.478047 \quad-2.152716$

H $\quad-0.757540 \quad 2.472104-1.297157$

C $\quad 0.274675 \quad 2.110556 \quad 1.255382$

H $\quad-0.757540 \quad 2.472104 \quad 1.297157$ 
H $\quad 0.775470 \quad 2.478047 \quad 2.152716$

C $\quad 0.948937 \quad 2.656097 \quad 0.000000$

H $\quad 0.924688 \quad 3.747250 \quad 0.000000$

H $2.0022792 .359163 \quad 0.000000$

H $\quad-1.4555390 .332626 \quad-0.000000$

$\mathrm{Br} \quad-0.386946-1.879068-0.000000$

\section{$1 \mathrm{Br}_{\mathrm{ax}}$ (MP2/aug-cc-pVTZ)}

Imaginary frequencies- 0

Electronic Energy (SCF) $=-2806.16998626$ a.u Electronic Energy (MP2) $=-2807.4643064259$ a.u ZPV corrected Energy = -2807.302194 a.u.

Enthalpy $=-2807.294438$ a.u.

Free Energy = -2807.334060 a.u.

$\begin{array}{llll}\text { C } & 0.436283 & -0.925553 & 0.000000 \\ \text { C } & 1.061820 & -0.351291 & 1.260734 \\ \text { H } & 2.096433 & -0.712086 & 1.292386 \\ \text { H } & 0.551932 & -0.743933 & 2.140976 \\ \text { C } & 1.061820 & -0.351291 & -1.260734 \\ \text { H } & 0.551932 & -0.743933 & -2.140976 \\ \text { H } & 2.096433 & -0.712086 & -1.292386 \\ \text { C } & 1.061820 & 1.173593 & -1.258549 \\ \text { H } & 1.570394 & 1.540808 & -2.151568 \\ \text { H } & 0.030560 & 1.531127 & -1.304805 \\ \text { C } & 1.061820 & 1.173593 & 1.258549 \\ \text { H } & 0.030560 & 1.531127 & 1.304805 \\ \text { H } & 1.570394 & 1.540808 & 2.151568 \\ \text { C } & 1.735069 & 1.715960 & -0.000000 \\ \text { H } & 1.711473 & 2.807106 & -0.000000 \\ \text { H } & 2.789597 & 1.420104 & -0.000000 \\ \text { H } & 0.493906 & -2.012087 & 0.000000 \\ \text { Br } & -1.485868 & -0.573058 & 0.000000\end{array}$

\section{$11_{\text {eq }}$ (MP2/aug-cc-pVTZ)}

Imaginary frequencies- 0

Electronic Energy (SCF) $=-528.368149474$ a.u

Electronic Energy (MP2) $=-529.6456523071$ a.u

ZPV corrected Energy $=-529.484195$ a.u.

Enthalpy $=-529.476177$ a.u.

Free Energy $=-529.517071$ a.u.

$\begin{array}{cccc}\text { C } & -0.317517 & 0.509121 & 0.000000 \\ \text { C } & 0.355208 & 1.025849 & 1.259271 \\ \text { H } & 1.384192 & 0.655629 & 1.287200 \\ \text { H } & -0.152287 & 0.640015 & 2.144186 \\ \text { C } & 0.355208 & 1.025849 & -1.259271 \\ \text { H } & -0.152287 & 0.640015 & -2.144186 \\ \text { H } & 1.384192 & 0.655629 & -1.287200 \\ \text { C } & 0.355208 & 2.555779 & -1.254972 \\ \text { H } & 0.855429 & 2.923852 & -2.152668 \\ \text { H } & -0.677292 & 2.916700 & -1.295348 \\ \text { C } & 0.355208 & 2.555779 & 1.254972 \\ \text { H } & -0.677292 & 2.916700 & 1.295348 \\ \text { H } & 0.855429 & 2.923852 & 2.152668 \\ \text { C } & 1.030600 & 3.100035 & 0.000000 \\ \text { H } & 1.008617 & 4.191282 & 0.000000 \\ \text { H } & 2.083423 & 2.801087 & 0.000000 \\ \text { H } & -1.375962 & 0.774880 & -0.000000 \\ \text { I } & -0.327163 & -1.635360 & -0.000000\end{array}$

\section{$1 \mathrm{I}_{\mathrm{ax}}$ (MP2/aug-cc-pVTZ)}

Imaginary frequencies- 0

Electronic Energy (SCF) $=-528.364996069$ a.u

Electronic Energy (MP2) $=-529.6457039628$ a.u ZPV corrected Energy $=-529.484178$ a.u.

Enthalpy $=-529.476298$ a.u.

Free Energy $=-529.516826$ a.u.

C $0.812688-0.819016 \quad 0.000000$

C $1.439362 \quad-0.241179 \quad 1.260077$

H $\quad 2.476703 \quad-0.597264 \quad 1.284312$

H $\quad 0.940747 \quad-0.634087 \quad 2.146677$

C $1.439362-0.241179-1.260077$

H $\quad 0.940747 \quad-0.634087 \quad-2.146677$

H $\quad 2.476703 \quad-0.597264-1.284312$

C $1.439362 \quad 1.283539-1.259639$

H $\quad 1.950332 \quad 1.650005-2.151700$

H $\quad 0.407925 \quad 1.641638-1.309862$

C $1.439362 \quad 1.283539 \quad 1.259639$

H $\quad 0.407925 \quad 1.641638 \quad 1.309862$

H $\quad 1.950332 \quad 1.650005 \quad 2.151700$

C $2.109174 \quad 1.827949 \quad 0.000000$

H $\quad 2.0816112 .9190710 .000000$

H $\quad 3.164860 \quad 1.536519 \quad 0.000000$

H $\quad 0.874871-1.905695 \quad 0.000000$

I $-1.316012-0.476083-0.000000$

\section{$1 \mathrm{CCH}_{\text {eq }}$ (MP2/aug-cc-pVTZ)}

Imaginary frequencies- 0

Electronic Energy (SCF) = -309.983503270 a.u Electronic Energy (MP2) = -311.3383296115 a.u ZPV corrected Energy $=-311.158313$ a.u. Enthalpy $=-311.149550$ a.u.

Free Energy = -311.190129 a.u.

$\begin{array}{rrrr}\text { C } & 0.549057 & 0.592555 & -0.000000 \\ \text { C } & -0.133068 & 0.042180 & 1.260022 \\ \text { H } & -1.161774 & 0.414437 & 1.283635 \\ \text { H } & 0.370139 & 0.430817 & 2.146964 \\ \text { C } & -0.133068 & 0.042180 & -1.260022 \\ \text { H } & 0.370139 & 0.430817 & -2.146964 \\ \text { H } & -1.161774 & 0.414437 & -1.283635 \\ \text { C } & -0.133068 & -1.483248 & -1.256294 \\ \text { H } & -0.634322 & -1.857197 & -2.151041 \\ \text { H } & 0.900146 & -1.842978 & -1.297158 \\ \text { C } & -0.133068 & -1.483248 & 1.256294 \\ \text { H } & 0.900146 & -1.842978 & 1.297158 \\ \text { H } & -0.634322 & -1.857197 & 2.151041 \\ \text { C } & -0.805231 & -2.029849 & 0.000000 \\ \text { H } & -0.779808 & -3.121182 & 0.000000 \\ \text { H } & -1.859279 & -1.734547 & 0.000000 \\ \text { H } & 1.589646 & 0.247018 & -0.000000 \\ \text { C } & 0.549390 & 2.050458 & -0.000000 \\ \text { C } & 0.508484 & 3.267203 & -0.000000 \\ \text { H } & 0.484511 & 4.329160 & -0.000000\end{array}$

$1 \mathrm{CCH}_{\mathrm{ax}}$ (MP2/aug-cc-pVTZ)

Imaginary frequencies- 0

Electronic Energy (SCF) = -309.981162355 a.u

Electronic Energy (MP2) $=-311.3386623888$ a.u 
ZPV corrected Energy $=-311.158498$ a.u. Enthalpy $=-311.149815$ a.u.

Free Energy = -311.190146 a.u.

C $\quad-0.099053 \quad-1.157166 \quad 0.000000$

$\begin{array}{llll}\text { C } & 0.583050 & -0.597148 & 1.259199\end{array}$

H $\quad 1.614558 \quad-0.9625601 .273519$

H $\quad 0.084377 \quad-0.984030 \quad 2.149395$

$\begin{array}{llll}\text { C } & 0.583050 & -0.597148 & -1.259199\end{array}$

H $\quad 0.084377-0.984030-2.149395$

H $\quad 1.614558-0.962560-1.273519$

C $\quad 0.583050 \quad 0.928001 \quad-1.258215$

H $1.088999 \quad 1.298873-2.151675$

H $\quad-0.449805 \quad 1.284746-1.301702$

$\begin{array}{llll}\text { C } & 0.583050 & 0.928001 & 1.258215\end{array}$

H $\quad-0.449805 \quad 1.284746 \quad 1.301702$

H $\quad 1.088999 \quad 1.298873 \quad 2.151675$

C $1.255186 \quad 1.472779-0.000000$

H $\quad 1.229916 \quad 2.564173-0.000000$

H $\quad 2.310078 \quad 1.178268-0.000000$

$\begin{array}{llll}\text { H } & 0.000943 & -2.246061 & 0.000000\end{array}$

C $-1.525463-0.8383150 .000000$

C $-2.708520-0.549297 \quad 0.000000$

H $\quad-3.743302 \quad-0.308680 \quad 0.000000$

\section{$1 \mathrm{CN}_{\text {eq }}$ (MP2/aug-cc-pVTZ)}

Imaginary frequencies- 0

Electronic Energy (SCF) = -326.048710101 a.u Electronic Energy (MP2) $=-327.4382490243$ a.u ZPV corrected Energy $=-327.268433$ a.u.

Enthalpy $=-327.260039$ a.u.

Free Energy = -327.300061 a.u.

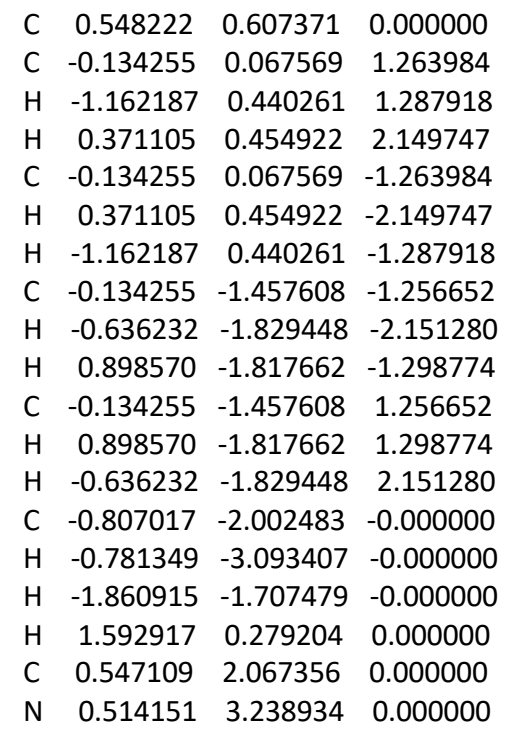

$1 \mathrm{CN}_{\mathrm{ax}}$ (MP2/aug-cc-pVTZ)

Imaginary frequencies- 0

Electronic Energy (SCF) $=-326.047036878$ a.u Electronic Energy (MP2) $=-327.4389537904$ a.u ZPV corrected Energy $=-327.269005$ a.u.

Enthalpy $=-327.260685$ a.u.

Free Energy $=-327.300491$ a.u.
C $\quad-0.117032-1.159785 \quad 0.000000$

C $0.556944 \quad-0.600726 \quad 1.262733$

H $\quad 1.586181 \quad-0.970103 \quad 1.275546$

H $\quad 0.057204 \quad-0.988097 \quad 2.151674$

C $0.556944-0.600726-1.262733$

H $\quad 0.057204 \quad-0.988097 \quad-2.151674$

H $\quad 1.586181-0.970103-1.275546$

C $0.556944 \quad 0.924529 \quad-1.258685$

H $\quad 1.063336 \quad 1.293793 \quad-2.151941$

H $\quad-0.475174 \quad 1.283972-1.305171$

C $\quad 0.556944 \quad 0.924529 \quad 1.258685$

H $\quad-0.475174 \quad 1.283972 \quad 1.305171$

H $\quad 1.063336 \quad 1.293793 \quad 2.151941$

C $1.228741 \quad 1.468511-0.000000$

H $\quad 1.201198 \quad 2.559351-0.000000$

H $\quad 2.283866 \quad 1.175859-0.000000$

H $-0.048221 \quad-2.2498810 .000000$

C $-1.539655-0.815856 \quad 0.000000$

$\begin{array}{llll}\text { N } & -2.671275 & -0.509617 & 0.000000\end{array}$

\section{$1 \mathbf{M e}_{\text {eq }}$ (MP2/aug-cc-pVTZ)}

Imaginary frequencies- 0

Electronic Energy (SCF) = -273.335961006 a.u Electronic Energy (MP2) $=-274.5755888661$ a.u ZPV corrected Energy = -274.376004 a.u. Enthalpy $=-274.367943$ a.u.

Free Energy = -274.406541 a.u.

$\begin{array}{llll}\text { C } & 0.579823 & 0.889483 & 0.000000\end{array}$

C $\quad-0.091285 \quad 0.325739 \quad 1.250814$

H $\quad-1.125907 \quad 0.687776 \quad 1.281988$

$\begin{array}{llll}\mathrm{H} & 0.406181 & 0.712137 & 2.144418\end{array}$

C $\quad-0.091285 \quad 0.325739-1.250814$

H $\quad 0.4061810 .712137 \quad-2.144418$

H $\quad-1.125907 \quad 0.687776-1.281988$

C $-0.091285-1.200529-1.256430$

H $\quad-0.592493 \quad-1.576410 \quad-2.150804$

H $\quad 0.942450-1.558840-1.296870$

C $-0.091285-1.200529 \quad 1.256430$

H $\quad 0.942450 \quad-1.558840 \quad 1.296870$

H $\quad-0.592493 \quad-1.576410 \quad 2.150804$

C $-0.761440-1.749682-0.000000$

H $\quad-0.734083-2.841224-0.000000$

H $-1.816480-1.456850-0.000000$

H $\quad 1.624984 \quad 0.555348 \quad 0.000000$

C $\quad 0.553921 \quad 2.411588 \quad 0.000000$

H $\quad 1.049848 \quad 2.815917 \quad 0.883692$

H $\quad \begin{array}{llll}H .049848 & 2.815917 & -0.883692\end{array}$

H $\quad-0.477578 \quad 2.770709 \quad 0.000000$

\section{Me (MP2/aug-cc-pVTZ)}

Imaginary frequencies- 0

Electronic Energy (SCF) $=-273.331796080$ a.u Electronic Energy (MP2) $=-274.5728967381$ a.u ZPV corrected Energy = -274.372981 a.u.

Enthalpy $=-274.364985$ a.u.

Free Energy $=-274.403408$ a.u.

C $-0.326425 \quad-1.180962 \quad 0.000000$

H $\quad-0.151932 \quad-2.261248 \quad 0.000000$

C $0.333833 \quad-0.598782 \quad 1.254411$ 


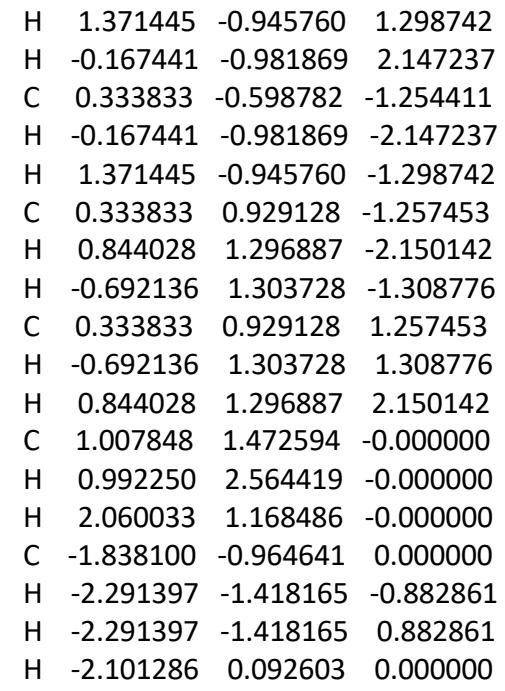

\section{Et $t_{\text {eq }}$ A (MP2/aug-cc-pVTZ)}

Imaginary frequencies- 0

Electronic Energy (SCF) $=-312.381627188$ a.u Electronic Energy (MP2) $=-313.8000617504$ a.u ZPV corrected Energy $=-313.571736$ a.u. Enthalpy $=-313.562297$ a.u.

Free Energy = -313.604479 a.u.

$\begin{array}{lrrr}\text { C } & 0.558467 & -0.254378 & 0.229498 \\ \text { C } & 0.067694 & 1.096274 & -0.290645 \\ \text { H } & 0.083870 & 1.070344 & -1.387208 \\ \text { H } & 0.745763 & 1.893985 & 0.018594 \\ \text { C } & -0.430342 & -1.350874 & -0.164381 \\ \text { H } & -0.090038 & -2.311700 & 0.231237 \\ \text { H } & -0.431329 & -1.440499 & -1.257273 \\ \text { C } & -1.846787 & -1.047949 & 0.315518 \\ \text { H } & -2.529881 & -1.841356 & 0.005009 \\ \text { H } & -1.858296 & -1.028208 & 1.410104 \\ \text { C } & -1.349110 & 1.407712 & 0.186552 \\ \text { H } & -1.345122 & 1.503207 & 1.277279 \\ \text { H } & -1.678530 & 2.368725 & -0.214128 \\ \text { C } & -2.321147 & 0.302201 & -0.213352 \\ \text { H } & -3.324731 & 0.525449 & 0.154447 \\ \text { H } & -2.383733 & 0.257328 & -1.305569 \\ \text { H } & 0.587575 & -0.203627 & 1.326892 \\ \text { C } & 1.961982 & -0.593513 & -0.267177 \\ \text { H } & 1.946522 & -0.622303 & -1.361610 \\ \text { C } & 3.042481 & 0.371056 & 0.209443 \\ \text { H } & 4.031690 & 0.034864 & -0.100755 \\ \text { H } & 2.895011 & 1.372346 & -0.192800 \\ \text { H } & 3.040439 & 0.443750 & 1.298274 \\ \text { H } & 2.211367 & -1.605480 & 0.064779\end{array}$

\section{Et $_{\text {eq }}$ B (MP2/aug-cc-pVTZ)}

Imaginary frequencies- 0

Electronic Energy (SCF) = -312.379593090 a.u Electronic Energy (MP2) $=-313.7988594231$ a.u ZPV corrected Energy $=-313.570337$ a.u.

Enthalpy $=-313.560939$ a.u.

Free Energy = -313.603070 a.u.

C $\quad 0.671333 \quad 0.4726890 .000000$

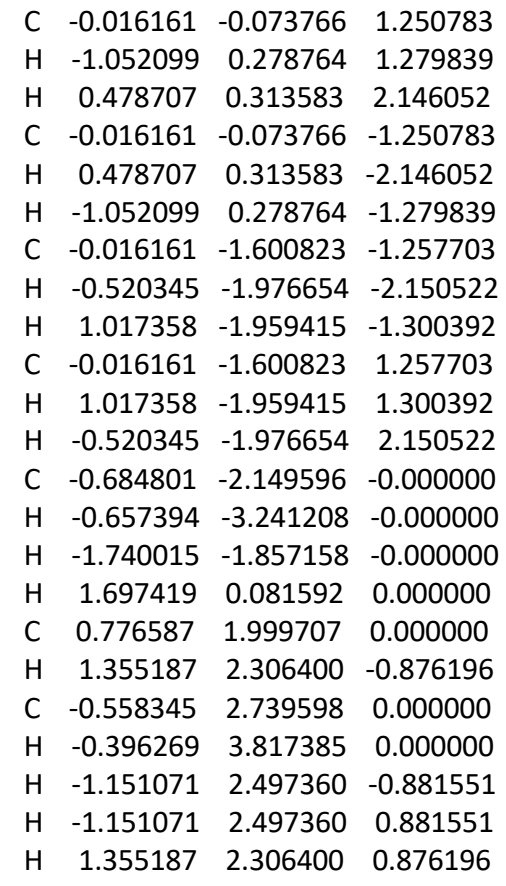

\section{Et ${ }_{a x}$ C (MP2/aug-cc-pVTZ)}

Imaginary frequencies- 0

Electronic Energy (SCF) $=-312.377157945$ a.u Electronic Energy (MP2) $=-313.7977319886$ a.u ZPV corrected Energy = -313.569028 a.u. Enthalpy $=-313.559717$ a.u.

Free Energy $=-313.601662$ a.u.

C $\quad-0.559521 \quad-0.415801 \quad 0.693352$

H $\quad-1.120846 \quad-0.690951 \quad 1.593735$

$\begin{array}{llll}\text { C } & -0.056665 & 1.020777 & 0.872589\end{array}$

H $\quad 0.491750 \quad 1.077846 \quad 1.818795$

H $\quad-0.895706 \quad 1.713705 \quad 0.958845$

C $0.640425-1.3619620 .574659$

H $\quad 0.289735 \quad-2.3855020 .416730$

H $\quad 1.188744 \quad-1.356109 \quad 1.522524$

$\begin{array}{llll}\text { C } & 1.598137 & -0.950563 & -0.543449\end{array}$

H $2.455591-1.626655-0.562597$

H $\quad 1.103683-1.048416-1.513509$

C $\quad 0.879951 \quad 1.446224 \quad-0.256358$

H $\quad 0.339169 \quad 1.457983 \quad-1.207203$

H $\quad 1.229746 \quad 2.466353-0.084422$

C $2.066933 \quad 0.490708-0.357800$

H $\quad 2.726545 \quad 0.784916-1.176828$

H $\quad 2.6539890 .558190 \quad 0.564330$

C $-1.517122-0.577873-0.490488$

H $\quad-1.007512-0.346388-1.427907$

C $\quad-2.776620 \quad 0.274003 \quad-0.377359$

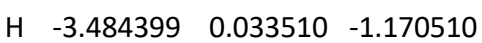

$\begin{array}{llll}\text { H } & -3.274197 & 0.104316 & 0.579088\end{array}$

H $\quad-2.550147 \quad 1.337179 \quad-0.451429$

H $\quad-1.799248-1.633059-0.550516$

\section{Et $_{\text {ax }}$ Cs (MP2/aug-cc-pVTZ)}

Imaginary frequencies- 0

Electronic Energy (SCF) $=-312.370578744$ a.u Electronic Energy (MP2) $=-313.7912346579$ a.u 
ZPV corrected Energy = -313.562496 a.u. Enthalpy $=-313.553033$ a.u.

Free Energy $=-313.596756$ a.u.

C $\quad 0.096244 \quad-1.169754 \quad 0.000000$

$\begin{array}{llll}\text { H } & 0.494138 & -2.190356 & 0.000000\end{array}$

C $0.648579-0.501061 \quad 1.269258$

H $1.687484-0.8239981 .395561$

H $\quad 0.101803 \quad-0.871852 \quad 2.141143$

C $0.648579-0.501061-1.269258$

H $\quad 0.101803 \quad-0.871852 \quad-2.141143$

H $1.687484-0.823998-1.395561$

C $0.648579 \quad 1.027951-1.249170$

H $1.1457491 .398842 \quad-2.148346$

H $\quad-0.370964 \quad 1.416625-1.271301$

C $\quad 0.648579 \quad 1.027951 \quad 1.249170$

H $\quad-0.370964 \quad 1.416625 \quad 1.271301$

H $\quad 1.145749 \quad 1.398842 \quad 2.148346$

C $1.347851 \quad 1.551918-0.000000$

H $\quad 1.363925 \quad 2.643597-0.000000$

H $\quad 2.390302 \quad 1.215367 \quad-0.000000$

C $-1.426148-1.370570 \quad 0.000000$

H $\quad-1.673582-1.980728-0.874097$

C $-2.329796-0.140592 \quad 0.000000$

$\begin{array}{llll}\text { H } & -3.375188 & -0.451540 & 0.000000\end{array}$

H $\quad-2.174476 \quad 0.4782350 .881992$

$\begin{array}{llll}H & -2.174476 & 0.478235 & -0.881992\end{array}$

H $\quad-1.673582 \quad-1.980728 \quad 0.874097$

\section{1iPr ${ }_{\text {eq }}$ A (MP2/aug-cc-pVTZ)}

Imaginary frequencies- 0

Electronic Energy (SCF) = -351.425325840 a.u

Electronic Energy (MP2) $=-353.0263339349$ a.u

ZPV corrected Energy = -352.769801 a.u.

Enthalpy $=-352.759005$ a.u.

Free Energy $=-352.804445$ a.u.

C $\quad 0.163722 \quad 0.317278 \quad 0.000000$

C $-0.179141-0.498112 \quad 1.249665$

H $\quad-1.268426 \quad-0.621209 \quad 1.293469$

$\begin{array}{llll}\text { H } & 0.115730 & 0.042602 & 2.149574\end{array}$

C $-0.179141-0.498112-1.249665$

H $\quad 0.115730 \quad 0.042602 \quad-2.149574$

H $\quad-1.268426-0.621209-1.293469$

C $0.483202 \quad-1.873814 \quad-1.248947$

H $\quad 0.201385-2.423673 \quad-2.149500$

H $\quad 1.570235-1.746401-1.282165$

C $\quad 0.483202 \quad-1.873814 \quad 1.248947$

H $\quad 1.570235-1.746401 \quad 1.282165$

H $\quad 0.201385-2.423673 \quad 2.149500$

C $0.113416-2.664344-0.000000$

H $\quad 0.610522-3.636545-0.000000$

H $\quad-0.964694-2.855426-0.000000$

H $\quad 1.249110 \quad 0.4984320 .000000$

C $\quad-0.529790 \quad 1.688761 \quad 0.000000$

H $-1.611791 \quad 1.506647 \quad 0.000000$

C $\quad-0.179141 \quad 2.505070 \quad 1.243187$

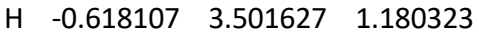

H $\quad-0.538516 \quad 2.043877 \quad 2.161070$

H $\quad 0.904569 \quad 2.622670 \quad 1.323234$

$\begin{array}{llll}\text { C } & -0.179141 & 2.505070 & -1.243187\end{array}$

H $\quad-0.538516 \quad 2.043877 \quad-2.161070$
$\begin{array}{llll}H & -0.618107 & 3.501627 & -1.180323\end{array}$

H $\quad 0.904569 \quad 2.622670 \quad-1.323234$

\section{1iPr $_{\text {eq }}$ B (MP2/aug-cc-pVTZ)}

Imaginary frequencies- 0

Electronic Energy (SCF) $=-351.425270557$ a.u

Electronic Energy (MP2) $=-353.0263623886$ a.u ZPV corrected Energy $=-352.769740$ a.u.

Enthalpy $=-352.758985$ a.u.

Free Energy $=-352.804128$ a.u.

C $0.272223 \quad-0.072309-0.433365$

C $\quad-0.595159-1.309669-0.200029$

H $\quad-0.504886-1.626066 \quad 0.844232$

H $\quad-0.231320-2.136282-0.816982$

$\begin{array}{llll}\text { C } & -0.243532 & 1.082363 & 0.426647\end{array}$

H $\quad 0.360985 \quad 1.9787590 .271389$

H $\quad-0.152751 \quad 0.812676 \quad 1.483834$

C $\quad-1.710034 \quad 1.382008 \quad 0.120759$

$\begin{array}{llll}H & -2.065234 & 2.204914 & 0.744794\end{array}$

H $-1.799396 \quad 1.709810-0.920016$

C $-2.064416-1.025836-0.503719$

H $\quad-2.169479-0.780851-1.565582$

H $\quad-2.668703 \quad-1.917118-0.321659$

$\begin{array}{llll}\text { C } & -2.576112 & 0.143683 & 0.332397\end{array}$

$\begin{array}{llll}\text { H } & -3.617922 & 0.360538 & 0.087349\end{array}$

H $\quad-2.548569-0.134483 \quad 1.391082$

H $\quad 0.1371010 .219521 \quad-1.484636$

C $1.769415 \quad-0.367934-0.250650$

H $\quad \begin{array}{llll}\text { H } & 1.977155 & -1.270327 & -0.837428\end{array}$

C $\quad 2.636510 \quad 0.756478-0.811081$

H $3.6927810 .486455 \quad-0.770299$

H $\quad 2.381457 \quad 0.971533 \quad-1.849989$

H $\quad 2.510861 \quad 1.674117 \quad-0.234500$

C $2.159287-0.6531201 .198000$

H $\quad 1.557647 \quad-1.448621 \quad 1.637483$

H $3.205169-0.958475 \quad 1.252810$

H $\quad 2.046011 \quad 0.239912 \quad 1.814369$

\section{1iPr $_{\text {ax }}$ C (MP2/aug-cc-pVTZ)}

Imaginary frequencies- 0

Electronic Energy (SCF) $=-351.420787888$ a.u Electronic Energy (MP2) = -353.0246790662 a.u ZPV corrected Energy = -352.767713 a.u. Enthalpy $=-352.757108$ a.u.

Free Energy = -352.802236 a.u.

C $\quad-0.222733 \quad 0.826655-0.000000$

H $\quad-0.336601 \quad 1.918486-0.000000$

C $-0.935324 \quad 0.272588 \quad 1.240680$

H $\quad-1.973876 \quad 0.619788 \quad 1.219971$

$\begin{array}{llll}H & -0.495602 & 0.670999 & 2.155761\end{array}$

C $\quad-0.935324 \quad 0.272588-1.240680$

H $\quad-0.495602 \quad 0.670999 \quad-2.155761$

H $-1.9738760 .619788-1.219971$

C $-0.935324-1.255156-1.262679$

H $\quad-1.463923-1.614358-2.148362$

H $\quad 0.090324 \quad-1.627017 \quad-1.340168$

C $\quad-0.935324-1.255156 \quad 1.262679$

H $0.090324-1.627017 \quad 1.340168$

H $\quad-1.463923 \quad-1.614358 \quad 2.148362$ 


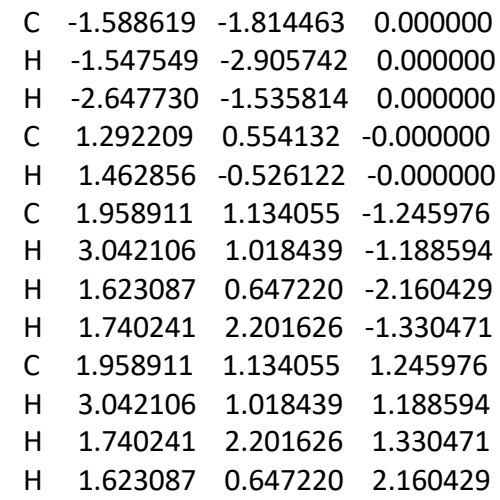

\section{$1 \mathrm{tBu}$ eq (MP2/aug-cc-pVTZ)}

Imaginary frequencies- 0

Electronic Energy (SCF) = -390.467990603 a.u Electronic Energy (MP2) $=-392.2546574020$ a.u ZPV corrected Energy $=-391.970245$ a.u. Enthalpy $=-391.958134$ a.u.

Free Energy $=-392.007348$ a.u.

$\begin{array}{llll}\text { C } & 0.329833 & -0.024284 & 0.000000\end{array}$

C $\quad-0.364879 \quad-0.582744 \quad 1.245988$

H $\quad-1.401513 \quad-0.230654 \quad 1.269515$

$\begin{array}{llll}\text { H } & 0.118400 & -0.218314 & 2.153271\end{array}$

C $\quad-0.364879-0.582744-1.245988$

H $\quad 0.118400 \quad-0.218314 \quad-2.153271$

H $\quad-1.401513 \quad-0.230654-1.269515$

C $\quad-0.364879-2.110621 \quad-1.251420$

H $\quad-0.864570 \quad-2.481330 \quad-2.149080$

H $\quad 0.669459-2.467428 \quad-1.292812$

C $\quad-0.364879-2.110621 \quad 1.251420$

H $\quad 0.669459 \quad-2.467428 \quad 1.292812$

H $\quad-0.864570-2.481330 \quad 2.149080$

C $-1.035285-2.665287-0.000000$

H $\quad-1.003898-3.756844-0.000000$

H $\quad-2.091132-2.375379-0.000000$

H $\quad 1.357973 \quad-0.4171710 .000000$

C $\quad 0.470542 \quad 1.518270 \quad 0.000000$

$\begin{array}{llll}\text { C } & 1.257488 & 1.965349 & 1.234812\end{array}$

H $\quad 1.4751143 .033400 \quad 1.171351$

H $\quad 0.701883 \quad 1.797657 \quad 2.156943$

H $\quad 2.207758 \quad 1.430458 \quad 1.304305$

C $1.257488 \quad 1.965349-1.234812$

H $\quad 0.701883 \quad 1.797657 \quad-2.156943$

H $\quad 1.475114 \quad 3.033400 \quad-1.171351$

H $\quad 2.207758 \quad 1.430458 \quad-1.304305$

C $\quad-0.886338 \quad 2.223400 \quad-0.000000$

$\begin{array}{llll}\text { H } & -1.471505 & 1.969928 & 0.884794\end{array}$

H $\quad-0.738272 \quad 3.305560 \quad 0.000000$

H $\quad-1.471505 \quad 1.969928 \quad-0.884794$

$1 \mathrm{tBu}$ ax (MP2/aug-cc-pVTZ)

Imaginary frequencies- 0

Electronic Energy (SCF) $=-390.457587850$ a.u

Electronic Energy (MP2) $=-392.2470456954$ a.u ZPV corrected Energy = -391.961813 a.u.

Enthalpy $=-391.950156$ a.u.

Free Energy $=-391.996625$ a.u.
C $\quad-0.172108-0.012538-0.847335$

H $\quad-0.618174 \quad-0.027436 \quad-1.849677$

C $0.740188-1.249047 \quad-0.794249$

H $\quad 1.315921-1.259592-1.727068$

H $\quad 0.148621 \quad-2.164512-0.795962$

C $\quad 0.697154 \quad 1.256277 \quad-0.777763$

H $\quad 0.069116 \quad 2.145522 \quad-0.687159$

H $1.218429 \quad 1.349855-1.736629$

C $1.760168 \quad 1.254706 \quad 0.325476$

$\begin{array}{llll}\text { H } & 2.388525 & 2.141318 & 0.211307\end{array}$

H $\quad 1.295192 \quad 1.328075 \quad 1.308560$

C $1.743504 \quad-1.251350 \quad 0.355430$

H $\quad 1.227880-1.284974 \quad 1.318162$

H $\quad 2.360387 \quad-2.151231 \quad 0.299419$

C $2.618903 \quad-0.004886 \quad 0.282787$

H $3.347358 \quad 0.006565 \quad 1.096165$

H $3.185687-0.027443 \quad-0.654206$

$\begin{array}{llll}\text { C } & -1.409901 & -0.007871 & 0.097587\end{array}$

$\begin{array}{llll}\text { C } & -2.450088 & 0.946148 & -0.501366\end{array}$

H $\quad-3.330671 \quad 0.998617 \quad 0.142306$

H $\quad-2.055346 \quad 1.957379-0.608136$

$\begin{array}{llll}\text { H } & -2.769574 & 0.599792 & -1.486315\end{array}$

C $-2.040962-1.400012 \quad 0.154473$

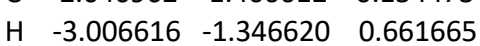

H $\quad-2.208414-1.798130-0.848957$

H $\quad-1.415923 \quad-2.105567 \quad 0.703360$

C $\quad-1.136071 \quad 0.454700 \quad 1.530657$

H $\quad-0.800964 \quad 1.491774 \quad 1.559577$

H $\quad-2.062787 \quad 0.393520 \quad 2.106461$

$\begin{array}{llll}\text { H } & -0.393371 & -0.163675 & 2.032947\end{array}$

\section{$1 \mathrm{Ph}_{\mathrm{eq}} \mathrm{A}$ (MP2/aug-cc-pVTZ)}

Imaginary frequencies- 0

Electronic Energy (SCF) $=-463.903726711$ a.u Electronic Energy (MP2) $=-465.9169807043$ a.u ZPV corrected Energy $=-465.664608$ a.u. Enthalpy $=-465.653387$ a.u.

Free Energy $=-465.700898$ a.u.

C $\quad 0.343138 \quad 0.655097 \quad 0.000000$

C $-0.334201 \quad 1.212503 \quad-1.256653$

H $\quad-1.366810 \quad 0.849604 \quad-1.292054$

H $\quad 0.1693140 .823948 \quad-2.145050$

C $\quad-0.334201 \quad 1.212503 \quad 1.256653$

H $\quad 0.169314 \quad 0.823948 \quad 2.145050$

H $\quad-1.366810 \quad 0.849604 \quad 1.292054$

C $\quad-0.334201 \quad 2.738738 \quad 1.256261$

H $\quad-0.834093 \quad 3.114848 \quad 2.151245$

H $\quad 0.699593 \quad 3.097250 \quad 1.294897$

C $\quad-0.334201 \quad 2.738738-1.256261$

H $\quad 0.699593 \quad 3.097250 \quad-1.294897$

H $\quad-0.834093 \quad 3.114848-2.151245$

C $\quad-1.006450 \quad 3.285103 \quad 0.000000$

H $\quad-0.982549 \quad 4.376675 \quad 0.000000$

H $\quad-2.060572 \quad 2.9889350 .000000$

H $\quad 1.382744 \quad 1.005524 \quad 0.000000$

C $0.369594-0.848860 \quad 0.000000$

C $1.582581 \quad-1.543644 \quad 0.000000$

C $-0.818080-1.590560 \quad 0.000000$

C $1.615612-2.937723 \quad 0.000000$

H $\quad 2.510931-0.983070 \quad 0.000000$

$\begin{array}{llll}\text { C } & -0.792605 & -2.983428 & 0.000000\end{array}$ 
$\begin{array}{llll}\text { H } & -1.773058 & -1.077903 & 0.000000\end{array}$

C $0.425657 \quad-3.662372 \quad 0.000000$

H $\quad 2.566580-3.4551320 .000000$

H $\quad-1.722170-3.5385410 .000000$

$\begin{array}{llll}\text { H } & 0.446237 & -4.744357 & 0.000000\end{array}$

\section{$1 \mathrm{Ph}_{\mathrm{ax}}$ B (MP2/aug-cc-pVTZ)}

Imaginary frequencies- 0

Electronic Energy (SCF) $=-463.895285137$ a.u

Electronic Energy (MP2) $=-465.9134446090$ a.u

ZPV corrected Energy = -465.660559 a.u.

Enthalpy $=-465.649578$ a.u.

Free Energy $=-465.696441$ a.u.

$\begin{array}{llll}\text { C } & -0.702277 & 0.327838 & 1.087899\end{array}$

C $-1.592803-0.9192961 .106752$

H $\quad-2.471831 \quad-0.686960 \quad 1.716402$

$\begin{array}{llll}\text { H } & -1.084644 & -1.746627 & 1.607096\end{array}$

C $\quad-1.453084 \quad 1.459093 \quad 0.366263$

$\begin{array}{llll}\text { H } & -0.848500 & 2.366211 & 0.338975\end{array}$

$\begin{array}{llll}\text { H } & -2.350933 & 1.698006 & 0.946000\end{array}$

C $-1.866010 \quad 1.047889-1.043161$

H $\quad-2.372141 \quad 1.877311 \quad-1.541349$

H $\quad-0.970893 \quad 0.817926 \quad-1.629861$

C $-2.068256-1.333708-0.286891$

H $\quad-1.218259-1.647013-0.897075$

H $\quad-2.736042 \quad-2.194249-0.205127$

C $-2.772044-0.178825 \quad-0.994119$

H $\quad-3.068468 \quad-0.478184-2.001379$

$\begin{array}{llll}\text { H } & -3.689245 & 0.075549 & -0.452442\end{array}$

H $\quad-0.548310 \quad 0.642543 \quad 2.126285$

C $0.6788450 .101195 \quad 0.496951$

C $1.169060-1.166947 \quad 0.165852$

$\begin{array}{llll}\text { C } & 1.539367 & 1.193425 & 0.317657\end{array}$

C $2.457119-1.336668-0.343630$

$\begin{array}{llll}\text { H } & 0.550598 & -2.042294 & 0.308335\end{array}$

C $2.826367 \quad 1.032111-0.189104$

H $\quad 1.206819 \quad 2.1876410 .589866$

C $3.290587-0.237423 \quad-0.529814$

H $\quad 2.805653 \quad-2.331018-0.592973$

H $\quad 3.466424 \quad 1.896500 \quad-0.313091$

H $4.288539-0.367446 \quad-0.927586$

\section{$1 \mathrm{Ph}_{\mathrm{ax}} \mathrm{C}$ (MP2/aug-cc-pVTZ)}

Imaginary frequencies- 0

Electronic Energy (SCF) $=-463.894768091 \mathrm{a} . \mathrm{u}$ Electronic Energy (MP2) $=-465.9108022626$ a.u ZPV corrected Energy $=-465.657957$ a.u.

Enthalpy $=-465.646794$ a.u.

Free Energy $=-465.695538$ a.u.

$\begin{array}{llll}\text { C } & 1.037942 & -0.683218 & 0.000000 \\ \text { C } & 1.651286 & -0.054109 & 1.263492 \\ \text { H } & 2.691769 & -0.391453 & 1.326199 \\ \text { H } & 1.137100 & -0.439087 & 2.147036 \\ \text { C } & 1.651286 & -0.054109 & -1.263492 \\ \text { H } & 1.137100 & -0.439087 & -2.147036 \\ \text { H } & 2.691769 & -0.391453 & -1.326199 \\ \text { C } & 1.651286 & 1.472559 & -1.261275 \\ \text { H } & 2.177036 & 1.833718 & -2.147968 \\ \text { H } & 0.630109 & 1.851153 & -1.335953\end{array}$

C $\quad 1.651286 \quad 1.472559 \quad 1.261275$

H $\quad 0.630109 \quad 1.851153 \quad 1.335953$

H $\quad 2.177036 \quad 1.833718 \quad 2.147968$

C $\quad 2.318714 \quad 2.015604 \quad 0.000000$

H $\quad 2.302352 \quad 3.1073810 .000000$

$\begin{array}{llll}H & 3.371680 & 1.713687 & 0.000000\end{array}$

H $\quad 1.340748 \quad-1.734677 \quad 0.000000$

C $-0.472581-0.7106550 .000000$

C $-1.289645 \quad 0.430055 \quad 0.000000$

C $-1.111712-1.958571 \quad 0.000000$

C $-2.680258 \quad 0.321541 \quad 0.000000$

H $\quad-0.853633 \quad 1.417048 \quad 0.000000$

$\begin{array}{llll}\text { C } & -2.500109 & -2.074310 & 0.000000\end{array}$

H $\quad-0.503899-2.856642 \quad 0.000000$

C $-3.293274-0.929228 \quad 0.000000$

H $\quad-3.283585 \quad 1.220715 \quad 0.000000$

H $\quad-2.958596 \quad-3.0550620 .000000$

H $\quad-4.372415 \quad-1.009823 \quad 0.000000$

\section{Ph ax $_{\text {(MP2/aug-cc-pVTZ) }}$}

Imaginary frequencies- 1

Electronic Energy (SCF) $=-463.894182196$ a.u Electronic Energy (MP2) $=-465.9131632196$ a.u ZPV corrected Energy $=-465.660376$ a.u. Enthalpy $=-465.650287$ a.u.

Free Energy $=-465.694463$ a.u.

C $-1.315484-0.375952-0.000000$

C $-1.194913-1.272305 \quad 1.239980$

H $\quad-2.014878-1.997000 \quad 1.208574$

H $\quad-1.332349-0.699645 \quad 2.157982$

C $-1.194913-1.272305-1.239980$

H $-1.332349-0.699645 \quad-2.157982$

H $\quad-2.014878-1.997000 \quad-1.208574$

C $0.133243-2.025563 \quad-1.258824$

H $0.194556-2.653639-2.150181$

H $\quad 0.957561 \quad-1.308837 \quad-1.314375$

C $0.133243 \quad-2.025563 \quad 1.258824$

H $\quad 0.957561 \quad-1.308837 \quad 1.314375$

H $\quad 0.194556 \quad-2.653639 \quad 2.150181$

C $0.288073-2.876502-0.000000$

H $\quad 1.256027-3.381736-0.000000$

H $\quad-0.479739-3.657445-0.000000$

H $\quad-2.318005 \quad 0.067059-0.000000$

C $-0.321622 \quad 0.776978 \quad 0.000000$

C $\quad 0.133243 \quad 1.347311 \quad 1.196224$

C $0.133243 \quad 1.347311 \quad-1.196224$

C $1.004896 \quad 2.435297 \quad 1.200353$

$\begin{array}{llll}\text { H } & -0.191285 & 0.944030 & 2.146017\end{array}$

C $1.004896 \quad 2.435297 \quad-1.200353$

H $\quad-0.191285 \quad 0.944030-2.146017$

C $\quad 1.448187 \quad 2.985190 \quad 0.000000$

H $\quad 1.337020 \quad 2.849972 \quad 2.143794$

H $\quad 1.337020 \quad 2.849972-2.143794$

H $\quad 2.127924 \quad 3.827215 \quad 0.000000$

1CF 3 еq (MP2/aug-cc-pVTZ)

Imaginary frequencies- 0

Electronic Energy (SCF) $=-570.046838753$ a.u Electronic Energy (MP2) $=-572.0225490248$ a.u ZPV corrected Energy = -571.845440 a.u. 
Enthalpy $=-571.835507$ a.u.

Free Energy $=-571.879655$ a.u.

$\begin{array}{llll}C & 0.390227 & 0.001568 & 0.000000\end{array}$

C $-0.289745-0.532856 \quad 1.259792$

$\begin{array}{llll}\text { H } & -1.320289 & -0.168114 & 1.285015\end{array}$

H $\quad 0.213473 \quad-0.151277 \quad 2.148681$

C $-0.289745-0.532856-1.259792$

H $\quad 0.213473 \quad-0.151277 \quad-2.148681$

H $\quad-1.320289-0.168114-1.285015$

C $-0.289745-2.059054-1.255718$

H $\quad-0.791884-2.430223-2.150558$

H $\quad 0.743074 \quad-2.419010-1.298007$

C $\quad-0.289745-2.059054 \quad 1.255718$

H $\quad 0.743074 \quad-2.419010 \quad 1.298007$

H $\quad-0.791884 \quad-2.430223 \quad 2.150558$

$\begin{array}{llll}\text { C } & -0.961671 & -2.606037 & 0.000000\end{array}$

H $\quad-0.934337 \quad-3.6970590 .000000$

H $\quad-2.016015-2.312295 \quad 0.000000$

H $\quad 1.439629-0.310064 \quad 0.000000$

C $\quad 0.420077 \quad 1.508067-0.000000$

F $\quad 1.057544 \quad 1.997744 \quad 1.081125$

F $\quad 1.057544 \quad 1.997744 \quad-1.081125$

F $\quad-0.816859 \quad 2.042067-0.000000$

\section{CF 3 3ax (MP2/aug-cc-pVTZ)}

Imaginary frequencies- 0

Electronic Energy (SCF) $=-570.041842191$ a.u Electronic Energy (MP2) $=-572.0188969276$ a.u ZPV corrected Energy $=-571.841532$ a.u.

Enthalpy $=-571.831694$ a.u.

Free Energy $=-571.875613$ a.u.

C $\quad-0.925325-0.020980-0.000000$

H $\quad-1.989723 \quad 0.227722 \quad-0.000000$

C $-0.616181-0.826266 \quad 1.267316$

H $\quad-1.368779-1.617289 \quad 1.329420$

$\begin{array}{llll}\text { H } & -0.746371 & -0.198232 & 2.149349\end{array}$

C $-0.616181-0.826266-1.267316$

H $\quad-0.746371-0.198232-2.149349$

H $\quad-1.368779-1.617289-1.329420$

C $0.767091-1.475183-1.256680$

H $\quad 0.875109-2.095066-2.148585$

H $\quad 1.542250-0.709030-1.306266$

C $0.767091 \quad-1.475183 \quad 1.256680$

H $\quad 1.542250 \quad-0.7090301 .306266$

$\begin{array}{llll}\text { H } & 0.875109 & -2.095066 & 2.148585\end{array}$

C $0.963590-2.318050 \quad 0.000000$

H $\quad 1.955833 \quad-2.772318 \quad 0.000000$

H $\quad 0.237237 \quad-3.137843 \quad 0.000000$

C $\quad-0.258613 \quad 1.334719-0.000000$

F $\quad-0.616181 \quad 2.055840-1.081218$

F $\quad-0.616181 \quad 2.055840 \quad 1.081218$

F $\quad 1.088297 \quad 1.284424-0.000000$

$\mathbf{1 C C l}_{\text {3еq }}$ (MP2/aug-cc-pVTZ)

Imaginary frequencies- 0

Electronic Energy (SCF) = -1650.12346763 a.u

Electronic Energy (MP2) $=-1651.9376630950$ a.u

ZPV corrected Energy = -1651.765997 a.u.

Enthalpy $=-1651.754853$ a.u.
Free Energy = -1651.802302 a.u.

$\begin{array}{llll}\text { C } & -0.412010 & 0.408217 & -0.000000\end{array}$

C $\quad 0.030137 \quad 1.165303 \quad 1.252821$

H $\quad 1.122058 \quad 1.221648 \quad 1.263488$

$\begin{array}{llll}H & -0.279284 & 0.628771 & 2.149207\end{array}$

C $\quad 0.030137 \quad 1.165303 \quad-1.252821$

H $\quad-0.2792840 .628771 \quad-2.149207$

H $\quad 1.122058 \quad 1.221648 \quad-1.263488$

C $-0.553461 \quad 2.576879-1.252513$

H $\quad-0.227232 \quad 3.105784 \quad-2.149707$

H $\quad-1.645294 \quad 2.515114 \quad-1.298948$

C $\quad-0.553461 \quad 2.576879 \quad 1.252513$

H $\quad-1.645294 \quad 2.515114 \quad 1.298948$

$\begin{array}{llll}H & -0.227232 & 3.105784 & 2.149707\end{array}$

C $\quad-0.144098 \quad 3.343243 \quad-0.000000$

H $\quad-0.590055 \quad 4.339486 \quad-0.000000$

H $\quad 0.942075 \quad 3.477657 \quad-0.000000$

$\begin{array}{llll}\text { H } & -1.506180 & 0.335743 & -0.000000\end{array}$

C $0.063093 \quad-1.043734 \quad 0.000000$

Cl $\quad-0.553461 \quad-1.903011 \quad 1.435500$

Cl $\quad-0.553461-1.903011-1.435500$

Cl $1.839371-1.149745 \quad 0.000000$

\section{$1 \mathrm{CCl}_{3 a x}$ (MP2/aug-cc-pVTZ)}

Imaginary frequencies- 0

Electronic Energy (SCF) = -1650.11347759 a.u

Electronic Energy (MP2) $=-1651.9300675904$ a.u

ZPV corrected Energy = -1651.758203 a.u.

Enthalpy $=-1651.747111$ a.u.

Free Energy = -1651.794478 a.u.

C $\quad-0.893534-0.430690 \quad-0.000000$

H $\quad-1.943438 \quad-0.121054 \quad 0.000000$

$\begin{array}{llll}\text { C } & -0.667010 & -1.273638 & 1.265844\end{array}$

H $\quad-1.535243-1.936045 \quad 1.340272$

H $\quad-0.687921 \quad-0.636495 \quad 2.149523$

C $-0.667010-1.273638-1.265844$

H $\quad-0.687921-0.636495-2.149523$

H $\quad-1.535243 \quad-1.936045 \quad-1.340272$

C $0.587073-2.146512-1.252777$

H $\quad 0.580775-2.773264-2.146981$

H $\quad 1.483079-1.528157 \quad-1.306145$

C $0.587073 \quad-2.146512 \quad 1.252777$

H $\quad 1.483079-1.528157 \quad 1.306145$

H $\quad 0.580775 \quad-2.773264 \quad 2.146981$

C $0.637108-3.013551-0.000000$

H $\quad 1.537027-3.631065-0.000000$

H $\quad-0.219490-3.696441-0.000000$

C $\quad-0.176365 \quad 0.923893 \quad 0.000000$

Cl $\quad-0.667010 \quad 1.867189 \quad-1.435311$

Cl $-0.667010 \quad 1.867189 \quad 1.435311$

Cl $\quad 1.598756 \quad 0.816231 \quad 0.000000$

\section{$1 \mathrm{CBr}_{\text {3eq }}$ (MP2/aug-cc-pVTZ)}

Imaginary frequencies- 0

Electronic Energy (SCF) = -7988.94257841 a.u

Electronic Energy (MP2) $=-7990.8982494080$ a.u ZPV corrected Energy = -7990.728615 a.u. Enthalpy $=-7990.716565$ a.u.

Free Energy $=-7990.767836$ a.u. 


$\begin{array}{lrrr}\mathrm{C} & -1.031024 & -0.000000 & 0.446692 \\ \mathrm{C} & -1.768356 & 1.250563 & -0.032581 \\ \mathrm{H} & -1.774235 & 1.255867 & -1.126025 \\ \mathrm{H} & -1.244345 & 2.146991 & 0.297045 \\ \mathrm{C} & -1.768355 & -1.250564 & -0.032581 \\ \mathrm{H} & -1.244344 & -2.146991 & 0.297046 \\ \mathrm{H} & -1.774234 & -1.255868 & -1.126025 \\ \mathrm{C} & -3.203846 & -1.252302 & 0.487983 \\ \mathrm{H} & -3.716838 & -2.149493 & 0.137168 \\ \mathrm{H} & -3.190306 & -1.300161 & 1.581381 \\ \mathrm{C} & -3.203847 & 1.252301 & 0.487982 \\ \mathrm{H} & -3.190308 & 1.300160 & 1.581380 \\ \mathrm{H} & -3.716840 & 2.149491 & 0.137166 \\ \mathrm{C} & -3.951948 & -0.000001 & 0.045979 \\ \mathrm{H} & -4.966589 & -0.000001 & 0.448360 \\ \mathrm{H} & -4.038649 & -0.000002 & -1.044992 \\ \mathrm{H} & -1.016105 & 0.000000 & 1.543839 \\ \mathrm{C} & 0.440958 & -0.000000 & 0.048537 \\ \mathrm{Br} & 1.338605 & 1.559477 & 0.773685 \\ \mathrm{Br} & 1.338607 & -1.559476 & 0.773686 \\ \mathrm{Br} & 0.659683 & -0.000000 & -1.874183\end{array}$

$\mathrm{1CBr}_{3 a x}$ (MP2/aug-cc-pVTZ)

Imaginary frequencies- 0

Electronic Energy (SCF) = -7988.93083628 a.u

Electronic Energy (MP2) $=-7990.8900699598 \mathrm{a} . \mathrm{u}$

ZPV corrected Energy = -7990.720280 a.u.

Enthalpy $=-7990.708258$ a.u.

Free Energy $=-7990.759509$ a.u.

C $\quad-0.941378 \quad-0.968460 \quad 0.000000$

H $\quad-1.986756 \quad-0.641336 \quad 0.000000$

C $\quad-0.735637 \quad-1.817562 \quad 1.266065$

H $\quad-1.627415 \quad-2.447067 \quad 1.348492$

H $\quad-0.727964 \quad-1.176048 \quad 2.146647$

C $\quad-0.735637-1.817562-1.266065$

H $\quad-0.727964-1.176048$ - 2.146647

H $\quad-1.627415-2.447067-1.348492$

C $0.485138-2.735540-1.251196$

H $\quad 0.458087 \quad-3.359497 \quad-2.146969$

H $\quad 1.402265-2.149202-1.300204$

C $0.485138 \quad-2.735540 \quad 1.251196$

H $1.402265-2.149202 \quad 1.300204$

H $\quad 0.458087 \quad-3.359497 \quad 2.146969$

C $0.500646 \quad-3.605368 \quad 0.000000$

H $\quad \begin{array}{llll}1.375705 & -4.257657 & 0.000000\end{array}$

H $\quad-0.382192-4.253995 \quad 0.000000$

C $\quad-0.205554 \quad 0.373497 \quad 0.000000$

$\begin{array}{llll}\mathrm{Br} & -0.735637 & 1.408051 & -1.558813\end{array}$

$\begin{array}{llll}\mathrm{Br} & -0.735637 & 1.408051 & 1.558813\end{array}$

$\begin{array}{llll}\mathrm{Br} & 1.724617 & 0.248351 & -0.000000\end{array}$

\section{Cl seq $_{\text {(MP2/aug-cc-pVTZ) }}$}

Imaginary frequencies- 0

Electronic Energy (SCF) = -1155.52879989 a.u

Electronic Energy (MP2) $=-1157.4455075544$ a.u

ZPV corrected Energy = -1157.277288 a.u.

Enthalpy $=-1157.264687$ a.u.

Free Energy $=-1157.318778$ a.u.
C $0.585557 \quad-1.333467 \quad 0.000000$

C $0.168725-2.113881 \quad 1.247335$

H $\quad-0.920687 \quad-2.213936 \quad 1.249689$

H $\quad 0.448196-1.565875 \quad 2.147086$

C $0.168725-2.113881-1.247335$

H $\quad 0.448196-1.565875 \quad-2.147086$

H $\quad-0.920687-2.213936-1.249689$

C $0.811303 \quad-3.499339-1.251724$

H $\quad 0.503910-4.038543 \quad-2.149564$

H $\quad 1.899342 \quad-3.391075-1.301449$

C $0.811303 \quad-3.49933901 .251724$

H $\quad 1.899342 \quad-3.391075 \quad 1.301449$

H $\quad 0.503910 \quad-4.038543 \quad 2.149564$

C $0.436453-4.2835920 .000000$

H $\quad 0.925810 \quad-5.2593340 .000000$

H $\quad-0.642889-4.464640 \quad 0.000000$

H $1.679928-1.237090 \quad 0.000000$

C $0.073822 \quad 0.107991-0.000000$

I $0.811303 \quad 1.166493 \quad 1.731630$

| $0.811303 \quad 1.166493 \quad-1.731630$

I $\begin{array}{llll}\text { I } & -2.078449 & 0.191410 & 0.000000\end{array}$

\section{$1 \mathrm{Cl}_{3 \mathrm{ax}}$ (MP2/aug-cc-pVTZ)}

Imaginary frequencies- 0

Electronic Energy (SCF) $=-1155.51513256$ a.u Electronic Energy (MP2) $=-1157.4357118808$ a.u ZPV corrected Energy = -1157.267419 a.u. Enthalpy $=-1157.254794$ a.u.

Free Energy = -1157.309035 a.u.

C $\quad-1.293537-0.969812-0.000000$

H $\quad-2.207516-0.363988-0.000000$

$\begin{array}{llll}\text { C } & -1.347201 & -1.846217 & 1.265516\end{array}$

H $\quad-2.389979-2.167085 \quad 1.358744$

H $\quad-1.133811-1.240225 \quad 2.146186$

C $-1.347201-1.846217-1.265516$

H $\quad-1.133811-1.240225-2.146186$

H $\quad-2.389979-2.167085-1.358744$

C $-0.473485-3.098773-1.248587$

H $\quad-0.690522 \quad-3.680251-2.147234$

H $\quad 0.581360 \quad-2.828493 \quad-1.293531$

$\begin{array}{llll}\text { C } & -0.473485 & -3.098773 & 1.248587\end{array}$

H $\quad 0.581360 \quad-2.828493 \quad 1.293531$

H $\quad-0.690522 \quad-3.6802512 .147234$

C $-0.733062-3.931789-0.000000$

H $\quad-0.105847 \quad-4.825079-0.000000$

H $-1.774776-4.271013-0.000000$

C $-0.210227 \quad 0.1190290 .000000$

I $-0.473485 \quad 1.395332-1.729965$

\begin{tabular}{llll}
\hline & -0.473485 & 1.395332 & 1.729965
\end{tabular}

I $1.826653-0.576937-0.000000$

\section{SiMe $_{\text {eq }}$ (MP2/aug-cc-pVTZ)}

Imaginary frequencies- 0

Electronic Energy (SCF) $=-641.572936530$ a.u Electronic Energy (MP2) $=-643.3057950816$ a.u ZPV corrected Energy = -643.033152 a.u.

Enthalpy $=-643.018583$ a.u.

Free Energy $=-643.072796$ a.u.

C $\quad-0.442773 \quad 0.218669-0.000000$ 


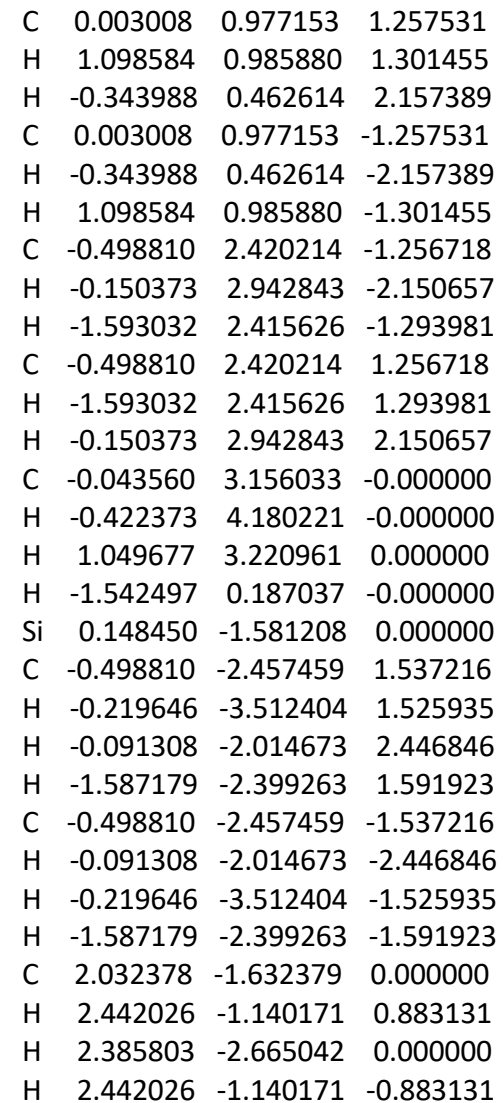

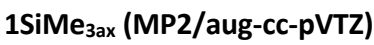

Imaginary frequencies- 0

Electronic Energy (SCF) $=-641.566649464$ a.u Electronic Energy (MP2) $=-643.3025974294$ a.u ZPV corrected Energy = -643.029374 a.u. Enthalpy $=-643.015164$ a.u.

Free Energy $=-643.068011$ a.u.

C $\quad 0.197780 \quad 0.001123 \quad-1.012960$

H $\quad-0.247565-0.000902-2.018106$

C $1.078604-1.250711-0.901152$

H $\quad 1.768769-1.276110-1.753541$

H $\quad 0.470946-2.156206-0.964969$

C $1.064785 \quad 1.264953-0.897114$

H $\quad 0.443483 \quad 2.165134 \quad-0.921468$

H $\quad 1.724952 \quad 1.320909-1.771134$

C $\quad 1.935314 \quad 1.263517 \quad 0.359610$

H $\quad 2.578056 \quad 2.1468190 .362298$

H $\quad \begin{array}{llll}1.304017 & 1.329786 & 1.247828\end{array}$

$\begin{array}{llll}\text { C } & 1.906697 & -1.251924 & 0.380465\end{array}$

H $\quad 1.241734 \quad-1.278115 \quad 1.249658$

$\begin{array}{llll}\text { H } & 2.524642 & -2.151276 & 0.428021\end{array}$

C $2.781971 \quad-0.003398 \quad 0.448699$

H $\quad 3.373220 \quad 0.001344 \quad 1.366823$

H $3.489878-0.023570 \quad-0.386667$

$\begin{array}{llll}\text { Si } & -1.362108 & -0.004590 & 0.080384\end{array}$

C $\quad-2.586741 \quad 1.191308$-0.709946

H $\quad-3.511255 \quad 1.240926-0.132326$

$\begin{array}{llll}H & -2.172582 & 2.199734 & -0.761635\end{array}$

H $\quad-2.8392620 .880213-1.724775$

C $-2.088100 \quad-1.743600 \quad 0.072944$

H $\quad-3.063208-1.752543 \quad 0.563052$
H $\quad-2.219950-2.112503-0.945656$

$\begin{array}{llll}\mathrm{H} & -1.441144 & -2.444282 & 0.603138\end{array}$

$\begin{array}{llll}\text { C } & -1.131089 & 0.534129 & 1.874562\end{array}$

H $\quad-0.812639 \quad 1.575003 \quad 1.943082$

$\begin{array}{llll}H & -2.090392 & 0.447957 & 2.390421\end{array}$

$\begin{array}{llll}\text { H } & -0.407507 & -0.080435 & 2.409932\end{array}$

Coordinates of fragments for dispersion computations:

Cy from $1 F_{\text {eq }}$

$\begin{array}{lrrr}\text { C } & 0.58133100 & 0.89502700 & 0.00000000 \\ \text { C } & -0.08987700 & 0.37832700 & 1.25306500 \\ \text { H } & -1.11733900 & 0.75186800 & 1.26794300 \\ \text { H } & 0.42032600 & 0.77538000 & 2.13151500 \\ \text { C } & -0.08987700 & 0.37832700 & -1.25306500 \\ \text { H } & 0.42032600 & 0.77538000 & -2.13151500 \\ \text { H } & -1.11733900 & 0.75186800 & -1.26794300 \\ \text { C } & -0.08987700 & -1.15039100 & -1.25637000 \\ \text { H } & -0.59062400 & -1.51903500 & -2.15265800 \\ \text { H } & 0.94174900 & -1.51338300 & -1.29917300 \\ \text { C } & -0.08987700 & -1.15039100 & 1.25637000 \\ \text { H } & 0.94174900 & -1.51338300 & 1.29917300 \\ \text { H } & -0.59062400 & -1.51903500 & 2.15265800 \\ \text { C } & -0.76212900 & -1.69809600 & 0.00000000 \\ \text { H } & -0.73289100 & -2.78914100 & 0.00000000 \\ \text { H } & -1.81660800 & -1.40565000 & 0.00000000 \\ \text { H } & 1.64061000 & 0.61951900 & 0.00000000 \\ \text { H } & 0.54769134 & 1.98450778 & 0.00000000\end{array}$

Cy from $1 \mathbf{F}_{\mathrm{ax}}$

$\begin{array}{lrrr}\text { C } & -0.34268500 & -1.18046700 & 0.00000000 \\ \text { C } & 0.28332500 & -0.61849700 & 1.26103700 \\ \text { H } & 1.31095900 & -0.99004300 & 1.31705600 \\ \text { H } & -0.25207900 & -1.00883100 & 2.12776700 \\ \text { C } & 0.28332500 & -0.61849700 & -1.26103700 \\ \text { H } & -0.25207900 & -1.00883100 & -2.12776700 \\ \text { H } & 1.31095900 & -0.99004300 & -1.31705600 \\ \text { C } & 0.28332500 & 0.90806700 & -1.25478800 \\ \text { H } & 0.77918300 & 1.28082600 & -2.15247400 \\ \text { H } & -0.74856400 & 1.26525300 & -1.28553300 \\ \text { C } & 0.28332500 & 0.90806700 & 1.25478800 \\ \text { H } & -0.74856400 & 1.26525300 & 1.28553300 \\ \text { H } & 0.77918300 & 1.28082600 & 2.15247400 \\ \text { C } & 0.96597900 & 1.44631800 & 0.00000000 \\ \text { H } & 0.95214900 & 2.53763300 & 0.00000000 \\ \text { H } & 2.01761000 & 1.14021900 & 0.00000000 \\ \text { H } & -0.30378400 & -2.27141500 & 0.00000000 \\ \text { H } & -1.40057762 & -0.91785852 & 0.00000000\end{array}$

Cy from $1 \mathrm{Cl}_{\text {eq }}$

$\begin{array}{lrrr}\text { C } & 0.50895600 & 0.50398000 & 0.00000000 \\ \text { C } & -0.16475000 & -0.01540800 & 1.25642000 \\ \text { H } & -1.19251500 & 0.35785200 & 1.27608600 \\ \text { H } & 0.34308400 & 0.37756500 & 2.13796800 \\ \text { C } & -0.16475000 & -0.01540800 & -1.25642000 \\ \text { H } & 0.34308400 & 0.37756500 & -2.13796800 \\ \text { H } & -1.19251500 & 0.35785200 & -1.27608600 \\ \text { C } & -0.16475000 & -1.54434200 & -1.25547800 \\ \text { H } & -0.66592300 & -1.91169000 & -2.15255900 \\ \text { H } & 0.86719000 & -1.90667600 & -1.29790300\end{array}$


Cy from $1 \mathrm{Cl}_{\mathrm{ax}}$

$\begin{array}{lrrr}\mathrm{C} & -0.02144900 & -1.08293600 & 0.00000000 \\ \mathrm{C} & 0.60519600 & -0.50723500 & 1.26078200 \\ \mathrm{H} & 1.63852500 & -0.86967300 & 1.29672200 \\ \mathrm{H} & 0.09032500 & -0.89947400 & 2.13847600 \\ \mathrm{C} & 0.60519600 & -0.50723500 & -1.26078200 \\ \mathrm{H} & 0.09032500 & -0.89947400 & -2.13847600 \\ \mathrm{H} & 1.63852500 & -0.86967300 & -1.29672200 \\ \mathrm{C} & 0.60519600 & 1.01815000 & -1.25731500 \\ \mathrm{H} & 1.11117400 & 1.38513800 & -2.15190000 \\ \mathrm{H} & -0.42542300 & 1.37775000 & -1.30044000 \\ \mathrm{C} & 0.60519600 & 1.01815000 & 1.25731500 \\ \mathrm{H} & -0.42542300 & 1.37775000 & 1.30044000 \\ \mathrm{H} & 1.11117400 & 1.38513800 & 2.15190000 \\ \mathrm{C} & 1.28154300 & 1.55916500 & 0.00000000 \\ \mathrm{H} & 1.26141200 & 2.65040000 & 0.00000000 \\ \mathrm{H} & 2.33511700 & 1.25974600 & 0.00000000 \\ \mathrm{H} & 0.04664400 & -2.16939100 & 0.00000000 \\ \mathrm{H} & -1.09257266 & -0.88096051 & 0.00000000\end{array}$

Cy from $1 \mathbf{B r}_{\text {eq }}$

$\begin{array}{lrrr}\text { C } & -0.39795000 & 0.06528900 & 0.00000000 \\ \text { C } & 0.27467500 & 0.58127400 & 1.25748400 \\ \text { H } & 1.30263400 & 0.20849700 & 1.28012200 \\ \text { H } & -0.23377800 & 0.19038600 & 2.13950900 \\ \text { C } & 0.27467500 & 0.58127400 & -1.25748400 \\ \text { H } & -0.23377800 & 0.19038600 & -2.13950900 \\ \text { H } & 1.30263400 & 0.20849700 & -1.28012200 \\ \text { C } & 0.27467500 & 2.11055600 & -1.25538200 \\ \text { H } & 0.77547000 & 2.47804700 & -2.15271600 \\ \text { H } & -0.75754000 & 2.47210400 & -1.29715700 \\ \text { C } & 0.27467500 & 2.11055600 & 1.25538200 \\ \text { H } & -0.75754000 & 2.47210400 & 1.29715700 \\ \text { H } & 0.77547000 & 2.47804700 & 2.15271600 \\ \text { C } & 0.94893700 & 2.65609700 & 0.00000000 \\ \text { H } & 0.92468800 & 3.74725000 & 0.00000000 \\ \text { H } & 2.00227900 & 2.35916300 & 0.00000000 \\ \text { H } & -1.45553900 & 0.33262600 & 0.00000000 \\ \text { H } & -0.39178129 & -1.02469354 & 0.00000000\end{array}$

Cy from $1 B r_{a x}$

$\begin{array}{llll}\text { C } & 0.43628300 & -0.92555300 & 0.00000000 \\ \text { C } & 1.06182000 & -0.35129100 & 1.26073400 \\ \text { H } & 2.09643300 & -0.71208600 & 1.29238600 \\ \text { H } & 0.55193200 & -0.74393300 & 2.14097600 \\ \text { C } & 1.06182000 & -0.35129100 & -1.26073400 \\ \text { H } & 0.55193200 & -0.74393300 & -2.14097600 \\ \text { H } & 2.09643300 & -0.71208600 & -1.29238600 \\ \text { C } & 1.06182000 & 1.17359300 & -1.25854900 \\ \text { H } & 1.57039400 & 1.54080800 & -2.15156800 \\ \text { H } & 0.03056000 & 1.53112700 & -1.30480500\end{array}$

\begin{tabular}{lrrr}
\multicolumn{4}{l}{ Cy from $1 \mathbf{l}_{\text {eq }}$} \\
$\mathrm{C}$ & -0.31751700 & 0.50912100 & 0.00000000 \\
$\mathrm{C}$ & 0.35520800 & 1.02584900 & 1.25927100 \\
$\mathrm{H}$ & 1.38419200 & 0.65562900 & 1.28720000 \\
$\mathrm{H}$ & -0.15228700 & 0.64001500 & 2.14418600 \\
$\mathrm{C}$ & 0.35520800 & 1.02584900 & -1.25927100 \\
$\mathrm{H}$ & -0.15228700 & 0.64001500 & -2.14418600 \\
$\mathrm{H}$ & 1.38419200 & 0.65562900 & -1.28720000 \\
$\mathrm{C}$ & 0.35520800 & 2.55577900 & -1.25497200 \\
$\mathrm{H}$ & 0.85542900 & 2.92385200 & -2.15266800 \\
$\mathrm{H}$ & -0.67729200 & 2.91670000 & -1.29534800 \\
$\mathrm{C}$ & 0.35520800 & 2.55577900 & 1.25497200 \\
$\mathrm{H}$ & -0.67729200 & 2.91670000 & 1.29534800 \\
$\mathrm{H}$ & 0.85542900 & 2.92385200 & 2.15266800 \\
$\mathrm{C}$ & 1.03060000 & 3.10003500 & 0.00000000 \\
$\mathrm{H}$ & 1.00861700 & 4.19128200 & 0.00000000 \\
$\mathrm{H}$ & 2.08342300 & 2.80108700 & 0.00000000 \\
$\mathrm{H}$ & -1.37596200 & 0.77488000 & 0.00000000 \\
$\mathrm{H}$ & -0.32241983 & -0.58086797 & 0.00000000
\end{tabular}

Cy from $1 \mathbf{l}_{\mathrm{ax}}$

$\begin{array}{llll}\text { C } & 0.81268800 & -0.81901600 & 0.00000000\end{array}$

$\begin{array}{llll}\text { C } & 1.43936200 & -0.24117900 & 1.26007700\end{array}$

$\begin{array}{llll}\mathrm{H} & 2.47670300 & -0.59726400 & 1.28431200\end{array}$

$\begin{array}{lllll}\text { H } & 0.94074700 & -0.63408700 & 2.14667700\end{array}$

C $\quad 1.43936200 \quad-0.24117900-1.26007700$

H $\quad 0.94074700 \quad-0.63408700 \quad-2.14667700$

H $\quad 2.47670300 \quad-0.59726400 \quad-1.28431200$

C $\quad 1.43936200 \quad 1.28353900 \quad-1.25963900$

$\mathrm{H} \quad 1.95033200 \quad 1.65000500 \quad-2.15170000$

H $\quad 0.40792500 \quad 1.64163800 \quad-1.30986200$

$\begin{array}{lllll}\text { C } & 1.43936200 & 1.28353900 & 1.25963900\end{array}$

$\begin{array}{llll}\text { H } & 0.40792500 & 1.64163800 & 1.30986200\end{array}$

$\begin{array}{llll}\mathrm{H} & 1.95033200 & 1.65000500 & 2.15170000\end{array}$

$\begin{array}{llll}\text { C } & 2.10917400 & 1.82794900 & 0.00000000\end{array}$

$\begin{array}{llll}\mathrm{H} & 2.08161100 & 2.91907100 & 0.00000000\end{array}$

$\begin{array}{llll}\mathrm{H} & 3.16486000 & 1.53651900 & 0.00000000\end{array}$

$\begin{array}{lllll}\mathrm{H} & 0.87487100 & -1.90569500 & 0.00000000\end{array}$

$\begin{array}{lllll}H & -0.26343704 & -0.64565254 & 0.00000000\end{array}$ 


$\begin{array}{lrrr}\mathrm{C} & -0.13306800 & -1.48324800 & 1.25629400 \\ \mathrm{H} & 0.90014600 & -1.84297800 & 1.29715800 \\ \mathrm{H} & -0.63432200 & -1.85719700 & 2.15104100 \\ \mathrm{C} & -0.80523100 & -2.02984900 & 0.00000000 \\ \mathrm{H} & -0.77980800 & -3.12118200 & 0.00000000 \\ \mathrm{H} & -1.85927900 & -1.73454700 & 0.00000000 \\ \mathrm{H} & 1.58964600 & 0.24701800 & 0.00000000 \\ \mathrm{H} & 0.54930597 & 1.68255497 & 0.00000000\end{array}$

$\mathbf{H C C H}$ from $1 \mathbf{C C H}_{\text {eq }}$

$\begin{array}{llll}\text { C } & 0.54939000 & 2.05045800 & 0.00000000\end{array}$

$\begin{array}{lllll}\text { C } & 0.50848400 & 3.26720300 & 0.00000000\end{array}$

$\begin{array}{lllll}\mathrm{H} & 0.48451100 & 4.32916000 & 0.00000000\end{array}$

$\begin{array}{llll}\mathrm{H} & 0.54914332 & 0.97045803 & 0.00000000\end{array}$

Cy from $1 \mathrm{CCH}_{\mathrm{ax}}$

$\begin{array}{lrrr}\mathrm{C} & -0.09905300 & -1.15716600 & 0.00000000 \\ \mathrm{C} & 0.58305000 & -0.59714800 & 1.25919900 \\ \mathrm{H} & 1.61455800 & -0.96256000 & 1.27351900 \\ \mathrm{H} & 0.08437700 & -0.98403000 & 2.14939500 \\ \mathrm{C} & 0.58305000 & -0.59714800 & -1.25919900 \\ \mathrm{H} & 0.08437700 & -0.98403000 & -2.14939500 \\ \mathrm{H} & 1.61455800 & -0.96256000 & -1.27351900 \\ \mathrm{C} & 0.58305000 & 0.92800100 & -1.25821500 \\ \mathrm{H} & 1.08899900 & 1.29887300 & -2.15167500 \\ \mathrm{H} & -0.44980500 & 1.28474600 & -1.30170200 \\ \mathrm{C} & 0.58305000 & 0.92800100 & 1.25821500 \\ \mathrm{H} & -0.44980500 & 1.28474600 & 1.30170200 \\ \mathrm{H} & 1.08899900 & 1.29887300 & 2.15167500 \\ \mathrm{C} & 1.25518600 & 1.47277900 & 0.00000000 \\ \mathrm{H} & 1.22991600 & 2.56417300 & 0.00000000 \\ \mathrm{H} & 2.31007800 & 1.17826800 & 0.00000000 \\ \mathrm{H} & 0.00094300 & -2.24606100 & 0.00000000 \\ \mathrm{H} & -1.16280059 & -0.91938235 & 0.00000000\end{array}$

HCCH from $1 \mathbf{C C H}_{\mathrm{ax}}$

C $\quad-1.52546300 \quad-0.83831500 \quad 0.00000000$

$\begin{array}{llll}\text { C } & -2.70852000 & -0.54929700 & 0.00000000\end{array}$

$\begin{array}{llll}\mathrm{H} & -3.74330200 & -0.30868000 & 0.00000000\end{array}$

$\begin{array}{llll}\mathrm{H} & -0.47147456 & -1.07391715 & 0.00000000\end{array}$

Cy from $1 \mathrm{CN}_{\text {eq }}$

$\begin{array}{lrrr}\text { C } & 0.54822200 & 0.60737100 & 0.00000000 \\ \text { C } & -0.13425500 & 0.06756900 & 1.26398400 \\ \text { H } & -1.16218700 & 0.44026100 & 1.28791800 \\ \text { H } & 0.37110500 & 0.45492200 & 2.14974700 \\ \text { C } & -0.13425500 & 0.06756900 & -1.26398400 \\ \text { H } & 0.37110500 & 0.45492200 & -2.14974700 \\ \text { H } & -1.16218700 & 0.44026100 & -1.28791800 \\ \text { C } & -0.13425500 & -1.45760800 & -1.25665200 \\ \text { H } & -0.63623200 & -1.82944800 & -2.15128000 \\ \text { H } & 0.89857000 & -1.81766200 & -1.29877400 \\ \text { C } & -0.13425500 & -1.45760800 & 1.25665200 \\ \text { H } & 0.89857000 & -1.81766200 & 1.29877400 \\ \text { H } & -0.63623200 & -1.82944800 & 2.15128000 \\ \text { C } & -0.80701700 & -2.00248300 & 0.00000000 \\ \text { H } & -0.78134900 & -3.09340700 & 0.00000000 \\ \text { H } & -1.86091500 & -1.70747900 & 0.00000000 \\ \text { H } & 1.59291700 & 0.27920400 & 0.00000000\end{array}$

HCN from $1 \mathbf{C N}_{\text {eq }}$

$\begin{array}{llll}\mathrm{C} & 0.54710900 & 2.06735600 & 0.00000000 \\ \mathrm{~N} & 0.51415100 & 3.23893400 & 0.00000000 \\ \mathrm{H} & 0.54793232 & 0.98735631 & 0.00000000\end{array}$

Cy from $1 \mathbf{C N}_{\mathrm{ax}}$

$\begin{array}{lrrr}\text { C } & -0.11703200 & -1.15978500 & 0.00000000 \\ \text { C } & 0.55694400 & -0.60072600 & 1.26273300 \\ \text { H } & 1.58618100 & -0.97010300 & 1.27554600 \\ \text { H } & 0.05720400 & -0.98809700 & 2.15167400 \\ \text { C } & 0.55694400 & -0.60072600 & -1.26273300 \\ \text { H } & 0.05720400 & -0.98809700 & -2.15167400 \\ \text { H } & 1.58618100 & -0.97010300 & -1.27554600 \\ \text { C } & 0.55694400 & 0.92452900 & -1.25868500 \\ \text { H } & 1.06333600 & 1.29379300 & -2.15194100 \\ \text { H } & -0.47517400 & 1.28397200 & -1.30517100 \\ \text { C } & 0.55694400 & 0.92452900 & 1.25868500 \\ \text { H } & -0.47517400 & 1.28397200 & 1.30517100 \\ \text { H } & 1.06333600 & 1.29379300 & 2.15194100 \\ \text { C } & 1.22874100 & 1.46851100 & 0.00000000 \\ \text { H } & 1.20119800 & 2.55935100 & 0.00000000 \\ \text { H } & 2.28386600 & 1.17585900 & 0.00000000 \\ \text { H } & -0.04822100 & -2.24988100 & 0.00000000 \\ \text { H } & -1.17651028 & -0.90364876 & 0.00000000\end{array}$

HCN from $1 \mathrm{CN}_{\mathrm{ax}}$

\begin{tabular}{|c|c|c|c|}
\hline C & -1.53965500 & -0.81585600 & 0.00000000 \\
\hline $\mathrm{N}$ & -2.67127500 & -0.50961700 & 0.00000000 \\
\hline $\mathrm{H}$ & -0.48989670 & -1.06964237 & 0.00000000 \\
\hline
\end{tabular}

Cy from $1 \mathrm{Me}_{\mathrm{eq}}$

$\begin{array}{lrrr}\text { C } & 0.57982300 & 0.88948300 & 0.00000000 \\ \text { C } & -0.09128500 & 0.32573900 & 1.25081400 \\ \text { H } & -1.12590700 & 0.68777600 & 1.28198800 \\ \text { H } & 0.40618100 & 0.71213700 & 2.14441800 \\ \text { C } & -0.09128500 & 0.32573900 & -1.25081400 \\ \text { H } & 0.40618100 & 0.71213700 & -2.14441800 \\ \text { H } & -1.12590700 & 0.68777600 & -1.28198800 \\ \text { C } & -0.09128500 & -1.20052900 & -1.25643000 \\ \text { H } & -0.59249300 & -1.57641000 & -2.15080400 \\ \text { H } & 0.94245000 & -1.55884000 & -1.29687000 \\ \text { C } & -0.09128500 & -1.20052900 & 1.25643000 \\ \text { H } & 0.94245000 & -1.55884000 & 1.29687000 \\ \text { H } & -0.59249300 & -1.57641000 & 2.15080400 \\ \text { C } & -0.76144000 & -1.74968200 & 0.00000000 \\ \text { H } & -0.73408300 & -2.84122400 & 0.00000000 \\ \text { H } & -1.81648000 & -1.45685000 & 0.00000000 \\ \text { H } & 1.62498400 & 0.55534800 & 0.00000000 \\ \text { H } & 0.56127691 & 1.97932521 & 0.00000000\end{array}$

$\begin{array}{lrcc}\mathrm{CH}_{4} \text { from } \mathbf{1} \mathbf{M e q}_{\text {eq }} & & \\ \mathrm{C} & 0.55392100 & 2.41158800 & 0.00000000 \\ \mathrm{H} & 1.04984800 & 2.81591700 & 0.88369200 \\ \mathrm{H} & 1.04984800 & 2.81591700 & -0.88369200 \\ \mathrm{H} & -0.47757800 & 2.77070900 & 0.00000000 \\ \mathrm{H} & 0.57246709 & 1.32174579 & 0.00000000\end{array}$




$\begin{array}{lrrr}\text { Cy from } 1 \mathrm{Me}_{\mathrm{ax}} & & \\ \mathrm{C} & -0.32642500 & -1.18096200 & 0.00000000 \\ \mathrm{H} & -0.15193200 & -2.26124800 & 0.00000000 \\ \mathrm{C} & 0.33383300 & -0.59878200 & 1.25441100 \\ \mathrm{H} & 1.37144500 & -0.94576000 & 1.29874200 \\ \mathrm{H} & -0.16744100 & -0.98186900 & 2.14723700 \\ \mathrm{C} & 0.33383300 & -0.59878200 & -1.25441100 \\ \mathrm{H} & -0.16744100 & -0.98186900 & -2.14723700 \\ \mathrm{H} & 1.37144500 & -0.94576000 & -1.29874200 \\ \mathrm{C} & 0.33383300 & 0.92912800 & -1.25745300 \\ \mathrm{H} & 0.84402800 & 1.29688700 & -2.15014200 \\ \mathrm{H} & -0.69213600 & 1.30372800 & -1.30877600 \\ \mathrm{C} & 0.33383300 & 0.92912800 & 1.25745300 \\ \mathrm{H} & -0.69213600 & 1.30372800 & 1.30877600 \\ \mathrm{H} & 0.84402800 & 1.29688700 & 2.15014200 \\ \mathrm{C} & 1.00784800 & 1.47259400 & 0.00000000 \\ \mathrm{H} & 0.99225000 & 2.56441900 & 0.00000000 \\ \mathrm{H} & 2.06003300 & 1.16848600 & 0.00000000 \\ \mathrm{H} & -1.40543320 & -1.02655571 & 0.00000000\end{array}$

$\begin{array}{lccl}\mathrm{CH}_{4} \text { from } & \text { 1Me } & & \\ \mathrm{ax} & -1.83810000 & -0.96464100 & 0.00000000 \\ \mathrm{C} & -2.29139700 & -1.41816500 & -0.88286100 \\ \mathrm{H} & -2.29139700 & -1.41816500 & 0.88286100 \\ \mathrm{H} & -2.10128600 & 0.09260300 & 0.00000000 \\ \mathrm{H} & -0.75909180 & -1.11904729 & 0.00000000 \\ \mathrm{H} & & & \end{array}$

Cy from 1Et eq $\mathrm{A}$

$\begin{array}{lrrr}\text { C } & 0.55846700 & -0.25437800 & 0.22949800 \\ \text { C } & 0.06769400 & 1.09627400 & -0.29064500 \\ \text { H } & 0.08387000 & 1.07034400 & -1.38720800 \\ \text { H } & 0.74576300 & 1.89398500 & 0.01859400 \\ \text { C } & -0.43034200 & -1.35087400 & -0.16438100 \\ \text { H } & -0.09003800 & -2.31170000 & 0.23123700 \\ \text { H } & -0.43132900 & -1.44049900 & -1.25727300 \\ \text { C } & -1.84678700 & -1.04794900 & 0.31551800 \\ \text { H } & -2.52988100 & -1.84135600 & 0.00500900 \\ \text { H } & -1.85829600 & -1.02820800 & 1.41010400 \\ \text { C } & -1.34911000 & 1.40771200 & 0.18655200 \\ \text { H } & -1.34512200 & 1.50320700 & 1.27727900 \\ \text { H } & -1.67853000 & 2.36872500 & -0.21412800 \\ \text { C } & -2.32114700 & 0.30220100 & -0.21335200 \\ \text { H } & -3.32473100 & 0.52544900 & 0.15444700 \\ \text { H } & -2.38373300 & 0.25732800 & -1.30556900 \\ \text { H } & 0.58757500 & -0.20362700 & 1.32689200 \\ \text { H } & 1.56035901 & -0.49646779 & -0.12505091\end{array}$

Ethane from 1Et $\mathrm{t}_{\text {eq }} \mathrm{A}$

$\begin{array}{llll}\text { C } & 1.96198200 & -0.59351300 & -0.26717700 \\ \text { H } & 1.94652200 & -0.62230300 & -1.36161000 \\ \text { C } & 3.04248100 & 0.37105600 & 0.20944300 \\ \text { H } & 4.03169000 & 0.03486400 & -0.10075500 \\ \text { H } & 2.89501100 & 1.37234600 & -0.19280000 \\ \text { H } & 3.04043900 & 0.44375000 & 1.29827400 \\ \text { H } & 2.21136700 & -1.60548000 & 0.06477900 \\ \text { H } & 0.96008999 & -0.35142321 & 0.08737191\end{array}$

$\begin{array}{lrrr}\text { Cy from } 1 \text { 1Et }_{\text {eq }} \text { B } & & \\ \text { C } & 0.67133300 & 0.47268900 & 0.00000000 \\ \text { C } & -0.01616100 & -0.07376600 & 1.25078300 \\ \text { H } & -1.05209900 & 0.27876400 & 1.27983900 \\ \text { H } & 0.47870700 & 0.31358300 & 2.14605200 \\ \text { C } & -0.01616100 & -0.07376600 & -1.25078300 \\ \text { H } & 0.47870700 & 0.31358300 & -2.14605200 \\ \text { H } & -1.05209900 & 0.27876400 & -1.27983900 \\ \mathrm{C} & -0.01616100 & -1.60082300 & -1.25770300 \\ \text { H } & -0.52034500 & -1.97665400 & -2.15052200 \\ \text { H } & 1.01735800 & -1.95941500 & -1.30039200 \\ \mathrm{C} & -0.01616100 & -1.60082300 & 1.25770300 \\ \mathrm{H} & 1.01735800 & -1.95941500 & 1.30039200 \\ \mathrm{H} & -0.52034500 & -1.97665400 & 2.15052200 \\ \mathrm{C} & -0.68480100 & -2.14959600 & 0.00000000 \\ \mathrm{H} & -0.65739400 & -3.24120800 & 0.00000000 \\ \mathrm{H} & -1.74001500 & -1.85715800 & 0.00000000 \\ \mathrm{H} & 1.69741900 & 0.08159200 & 0.00000000 \\ \mathrm{H} & 0.74628647 & 1.56010887 & 0.00000000\end{array}$

\section{Ethane from 1Et eq B}

$\begin{array}{llll}\text { C } & 0.77658700 & 1.99970700 & 0.00000000 \\ \text { H } & 1.35518700 & 2.30640000 & -0.87619600 \\ \text { C } & -0.55834500 & 2.73959800 & 0.00000000 \\ \text { H } & -0.39626900 & 3.81738500 & 0.00000000 \\ \text { H } & -1.15107100 & 2.49736000 & -0.88155100 \\ \text { H } & -1.15107100 & 2.49736000 & 0.88155100 \\ \text { H } & 1.35518700 & 2.30640000 & 0.87619600 \\ \text { H } & 0.70163353 & 0.91228713 & 0.00000000\end{array}$

\begin{tabular}{lrrr}
\multicolumn{4}{l}{ Cy from $1 \mathrm{Et}_{\mathrm{ax}} \mathrm{C}$} \\
$\mathrm{C}$ & -0.55952100 & -0.41580100 & 0.69335200 \\
$\mathrm{H}$ & -1.12084600 & -0.69095100 & 1.59373500 \\
$\mathrm{C}$ & -0.05666500 & 1.02077700 & 0.87258900 \\
$\mathrm{H}$ & 0.49175000 & 1.07784600 & 1.81879500 \\
$\mathrm{H}$ & -0.89570600 & 1.71370500 & 0.95884500 \\
$\mathrm{C}$ & 0.64042500 & -1.36196200 & 0.57465900 \\
$\mathrm{H}$ & 0.28973500 & -2.38550200 & 0.41673000 \\
$\mathrm{H}$ & 1.18874400 & -1.35610900 & 1.52252400 \\
$\mathrm{C}$ & 1.59813700 & -0.95056300 & -0.54344900 \\
$\mathrm{H}$ & 2.45559100 & -1.62665500 & -0.56259700 \\
$\mathrm{H}$ & 1.10368300 & -1.04841600 & -1.51350900 \\
$\mathrm{C}$ & 0.87995100 & 1.44622400 & -0.25635800 \\
$\mathrm{H}$ & 0.33916900 & 1.45798300 & -1.20720300 \\
$\mathrm{H}$ & 1.22974600 & 2.46635300 & -0.08442200 \\
$\mathrm{C}$ & 2.06693300 & 0.49070800 & -0.35780000 \\
$\mathrm{H}$ & 2.72654500 & 0.78491600 & -1.17682800 \\
$\mathrm{H}$ & 2.65398900 & 0.55819000 & 0.56433000 \\
$\mathrm{H}$ & -1.24117401 & -0.53116937 & -0.14934564
\end{tabular}

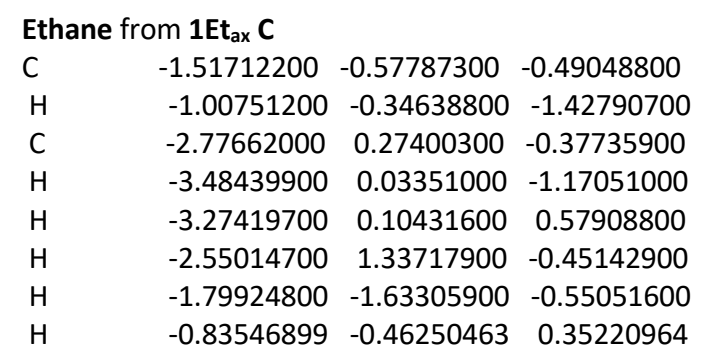


Cy from $1 \mathrm{Et}_{\mathrm{ax}} C_{\mathrm{s}}$

$\begin{array}{llll}\text { C } & 0.09624400 & -1.16975400 & 0.00000000 \\ \text { H } & 0.49413800 & -2.19035600 & 0.00000000 \\ \text { C } & 0.64857900 & -0.50106100 & 1.26925800 \\ \text { H } & 1.68748400 & -0.82399800 & 1.39556100 \\ \text { H } & 0.10180300 & -0.87185200 & 2.14114300 \\ \text { C } & 0.64857900 & -0.50106100 & -1.26925800 \\ \text { H } & 0.10180300 & -0.87185200 & -2.14114300 \\ \text { H } & 1.68748400 & -0.82399800 & -1.39556100 \\ \text { C } & 0.64857900 & 1.02795100 & -1.24917000 \\ \text { H } & 1.14574900 & 1.39884200 & -2.14834600 \\ \text { H } & -0.37096400 & 1.41662500 & -1.27130100 \\ \text { C } & 0.64857900 & 1.02795100 & 1.24917000 \\ \text { H } & -0.37096400 & 1.41662500 & 1.27130100 \\ \text { H } & 1.14574900 & 1.39884200 & 2.14834600 \\ \text { C } & 1.34785100 & 1.55191800 & 0.00000000 \\ \text { H } & 1.36392500 & 2.64359700 & 0.00000000 \\ \text { H } & 2.39030200 & 1.21536700 & 0.00000000 \\ \text { H } & -0.98439510 & -1.31229917 & 0.00000000\end{array}$

Ethane from $1 \mathrm{Et}_{\mathrm{ax}} C_{\mathrm{s}}$

$\begin{array}{llll}\text { C } & -1.42614800 & -1.37057000 & 0.00000000 \\ \text { H } & -1.67358200 & -1.98072800 & -0.87409700 \\ \text { C } & -2.32979600 & -0.14059200 & 0.00000000 \\ \text { H } & -3.37518800 & -0.45154000 & 0.00000000 \\ \text { H } & -2.17447600 & 0.47823500 & 0.88199200 \\ \text { H } & -2.17447600 & 0.47823500 & -0.88199200 \\ \text { H } & -1.67358200 & -1.98072800 & 0.87409700 \\ \text { H } & -0.34550890 & -1.22802483 & 0.00000000\end{array}$

$\begin{array}{lrrr}\text { Cy from 1iPreq } & \text { A } & & \\ \text { C } & 0.16372200 & 0.31727800 & 0.00000000 \\ \text { C } & -0.17914100 & -0.49811200 & 1.24966500 \\ \text { H } & -1.26842600 & -0.62120900 & 1.29346900 \\ \text { H } & 0.11573000 & 0.04260200 & 2.14957400 \\ \text { C } & -0.17914100 & -0.49811200 & -1.24966500 \\ \text { H } & 0.11573000 & 0.04260200 & -2.14957400 \\ \text { H } & -1.26842600 & -0.62120900 & -1.29346900 \\ \text { C } & 0.48320200 & -1.87381400 & -1.24894700 \\ \text { H } & 0.20138500 & -2.42367300 & -2.14950000 \\ \text { H } & 1.57023500 & -1.74640100 & -1.28216500 \\ \text { C } & 0.48320200 & -1.87381400 & 1.24894700 \\ \text { H } & 1.57023500 & -1.74640100 & 1.28216500 \\ \text { H } & 0.20138500 & -2.42367300 & 2.14950000 \\ \text { C } & 0.11341600 & -2.66434400 & 0.00000000 \\ \text { H } & 0.61052200 & -3.63654500 & 0.00000000 \\ \text { H } & -0.96469400 & -2.85542600 & 0.00000000 \\ \text { H } & 1.24911000 & 0.49843200 & 0.00000000 \\ \text { H } & -0.32814476 & 1.28998921 & 0.00000000\end{array}$

\section{Propane from $1 \mathrm{iPr}_{\mathrm{eq}} \mathbf{A}$}

$\begin{array}{llll}\text { C } & -0.52979000 & 1.68876100 & 0.00000000 \\ \text { H } & -1.61179100 & 1.50664700 & 0.00000000 \\ \text { C } & -0.17914100 & 2.50507000 & 1.24318700 \\ \text { H } & -0.61810700 & 3.50162700 & 1.18032300 \\ \text { H } & -0.53851600 & 2.04387700 & 2.16107000 \\ \text { H } & 0.90456900 & 2.62267000 & 1.32323400 \\ \text { C } & -0.17914100 & 2.50507000 & -1.24318700 \\ \text { H } & -0.53851600 & 2.04387700 & -2.16107000\end{array}$

$\begin{array}{lll}-0.61810700 & 3.50162700 & -1.18032300\end{array}$ $\begin{array}{lll}0.90456900 & 2.62267000 & -1.32323400\end{array}$ $\begin{array}{lll}-0.03792324 & 0.71604979 & 0.00000000\end{array}$

\begin{tabular}{|c|c|c|c|}
\hline \multicolumn{4}{|c|}{ Cy from $1 \mathrm{iPr}_{\text {eq }} \mathrm{B}$} \\
\hline C & 0.27222300 & -0.07230900 & -0.43336500 \\
\hline C & -0.59515900 & -1.30966900 & -0.20002900 \\
\hline $\mathrm{H}$ & -0.50488600 & -1.62606600 & 0.84423200 \\
\hline $\mathrm{H}$ & -0.23132000 & -2.13628200 & -0.81698200 \\
\hline C & -0.24353200 & 1.08236300 & 0.42664700 \\
\hline $\mathrm{H}$ & 0.36098500 & 1.97875900 & 0.27138900 \\
\hline $\mathrm{H}$ & -0.15275100 & 0.81267600 & 1.48383400 \\
\hline C & -1.71003400 & 1.38200800 & 0.12075900 \\
\hline $\mathrm{H}$ & -2.06523400 & 2.20491400 & 0.74479400 \\
\hline $\mathrm{H}$ & -1.79939600 & 1.70981000 & -0.92001600 \\
\hline C & -2.06441600 & -1.02583600 & -0.50371900 \\
\hline $\mathrm{H}$ & -2.16947900 & -0.78085100 & -1.56558200 \\
\hline $\mathrm{H}$ & -2.66870300 & -1.91711800 & -0.32165900 \\
\hline C & -2.57611200 & 0.14368300 & 0.33239700 \\
\hline $\mathrm{H}$ & -3.61792200 & 0.36053800 & 0.08734900 \\
\hline $\mathrm{H}$ & -2.54856900 & -0.13448300 & 1.39108200 \\
\hline $\mathrm{H}$ & 0.13710100 & 0.21952100 & -1.48463600 \\
\hline $\mathrm{H}$ & 1.33399361 & -0.28195875 & -0.30378815 \\
\hline
\end{tabular}

Propane from 1iPreq $B$

C $\quad 1.76941500 \quad-0.36793400 \quad-0.25065000$

H $\quad \begin{array}{llll}\text { H } & 1.97715500 & -1.27032700 & -0.83742800\end{array}$

$\begin{array}{lllll}\text { C } & 2.63651000 & 0.75647800 & -0.81108100\end{array}$

H $\quad 3.69278100 \quad 0.48645500 \quad-0.77029900$

H $\quad 2.38145700 \quad 0.97153300 \quad-1.84998900$

H $\quad 2.51086100 \quad 1.67411700 \quad-0.23450000$

$\begin{array}{llll}\text { C } & 2.15928700 & -0.65312000 & 1.19800000\end{array}$

H $\quad \begin{array}{llll}\mathrm{H} & 1.55764700 & -1.44862100 & 1.63748300\end{array}$

H $\quad 3.20516900-0.95847500 \quad 1.25281000$

$\begin{array}{llll}H & 2.04601100 & 0.23991200 & 1.81436900\end{array}$

$\begin{array}{llll}H & 0.70764439 & -0.15828425 & -0.38022685\end{array}$

$\begin{array}{lrrr}\text { Cy from } 1 \text { Pr }_{\mathrm{ax}} \mathrm{C} & & \\ \mathrm{C} & -0.22273300 & 0.82665500 & 0.00000000 \\ \mathrm{H} & -0.33660100 & 1.91848600 & 0.00000000 \\ \mathrm{C} & -0.93532400 & 0.27258800 & 1.24068000 \\ \mathrm{H} & -1.97387600 & 0.61978800 & 1.21997100 \\ \mathrm{H} & -0.49560200 & 0.67099900 & 2.15576100 \\ \mathrm{C} & -0.93532400 & 0.27258800 & -1.24068000 \\ \mathrm{H} & -0.49560200 & 0.67099900 & -2.15576100 \\ \mathrm{H} & -1.97387600 & 0.61978800 & -1.21997100 \\ \mathrm{C} & -0.93532400 & -1.25515600 & -1.26267900 \\ \mathrm{H} & -1.46392300 & -1.61435800 & -2.14836200 \\ \mathrm{H} & 0.09032400 & -1.62701700 & -1.34016800 \\ \mathrm{C} & -0.93532400 & -1.25515600 & 1.26267900 \\ \mathrm{H} & 0.09032400 & -1.62701700 & 1.34016800 \\ \mathrm{H} & -1.46392300 & -1.61435800 & 2.14836200 \\ \mathrm{C} & -1.58861900 & -1.81446300 & 0.00000000 \\ \mathrm{H} & -1.54754900 & -2.90574200 & 0.00000000 \\ \mathrm{H} & -2.64773000 & -1.53581400 & 0.00000000 \\ \mathrm{H} & 0.85004738 & 0.63367248 & 0.00000000\end{array}$

Propane from $1 \mathrm{iPr}_{\mathrm{ax}} \mathrm{C}$

$\begin{array}{lcll}\mathrm{C} & 1.29220900 & 0.55413200 & 0.00000000 \\ \mathrm{H} & 1.46285600 & -0.52612200 & 0.00000000\end{array}$ 


$\begin{array}{llll}\mathrm{C} & 1.95891100 & 1.13405500 & -1.24597600 \\ \mathrm{H} & 3.04210600 & 1.01843900 & -1.18859400 \\ \mathrm{H} & 1.62308700 & 0.64722000 & -2.16042900 \\ \mathrm{H} & 1.74024100 & 2.20162600 & -1.33047100 \\ \mathrm{C} & 1.95891100 & 1.13405500 & 1.24597600 \\ \mathrm{H} & 3.04210600 & 1.01843900 & 1.18859400 \\ \mathrm{H} & 1.74024100 & 2.20162600 & 1.33047100 \\ \mathrm{H} & 1.62308700 & 0.64722000 & 2.16042900 \\ \mathrm{H} & 0.21942862 & 0.74711452 & 0.00000000\end{array}$

\section{Cy from $1 \mathrm{tBu}_{\mathrm{eq}}$}

$\begin{array}{lrrr}\text { C } & 0.32983300 & -0.02428400 & 0.00000000 \\ \text { C } & -0.36487900 & -0.58274400 & 1.24598800 \\ \text { H } & -1.40151300 & -0.23065400 & 1.26951500 \\ \text { H } & 0.11840000 & -0.21831400 & 2.15327100 \\ \text { C } & -0.36487900 & -0.58274400 & -1.24598800 \\ \text { H } & 0.11840000 & -0.21831400 & -2.15327100 \\ \text { H } & -1.40151300 & -0.23065400 & -1.26951500 \\ \text { C } & -0.36487900 & -2.11062100 & -1.25142000 \\ \text { H } & -0.86457000 & -2.48133000 & -2.14908000 \\ \text { H } & 0.66945900 & -2.46742800 & -1.29281200 \\ \text { C } & -0.36487900 & -2.11062100 & 1.25142000 \\ \text { H } & 0.66945900 & -2.46742800 & 1.29281200 \\ \text { H } & -0.86457000 & -2.48133000 & 2.14908000 \\ \text { C } & -1.03528500 & -2.66528700 & 0.00000000 \\ \text { H } & -1.00389800 & -3.75684400 & 0.00000000 \\ \text { H } & -2.09113200 & -2.37537900 & 0.00000000 \\ \text { H } & 1.35797300 & -0.41717100 & 0.00000000 \\ \text { H } & 0.42884974 & 1.06120929 & 0.00000000\end{array}$

Iso-Butane from 1tBu eq
$\begin{array}{llll}\text { C } & 0.47054200 & 1.51827000 & 0.00000000 \\ \mathrm{C} & 1.25748800 & 1.96534900 & 1.23481200 \\ \mathrm{H} & 1.47511400 & 3.03340000 & 1.17135100 \\ \mathrm{H} & 0.70188300 & 1.79765700 & 2.15694300 \\ \mathrm{H} & 2.20775800 & 1.43045800 & 1.30430500 \\ \mathrm{C} & 1.25748800 & 1.96534900 & -1.23481200 \\ \mathrm{H} & 0.70188300 & 1.79765700 & -2.15694300 \\ \mathrm{H} & 1.47511400 & 3.03340000 & -1.17135100 \\ \mathrm{H} & 2.20775800 & 1.43045800 & -1.30430500 \\ \mathrm{C} & -0.88633800 & 2.22340000 & 0.00000000 \\ \mathrm{H} & -1.47150500 & 1.96992800 & 0.88479400 \\ \mathrm{H} & -0.73827200 & 3.30556000 & 0.00000000 \\ \mathrm{H} & -1.47150500 & 1.96992800 & -0.88479400 \\ \mathrm{H} & 0.37152526 & 0.43277671 & 0.00000000\end{array}$

Cy from $1 \mathrm{tBu}$ ax

$\begin{array}{llll}\text { C } & -0.17210800 & -0.01253800 & -0.84733500 \\ \text { H } & -0.61817400 & -0.02743600 & -1.84967700 \\ \text { C } & 0.74018800 & -1.24904700 & -0.79424900 \\ \text { H } & 1.31592100 & -1.25959200 & -1.72706800 \\ \text { H } & 0.14862100 & -2.16451200 & -0.79596200 \\ \text { C } & 0.69715400 & 1.25627700 & -0.77776300 \\ \text { H } & 0.06911600 & 2.14552200 & -0.68715900 \\ \text { H } & 1.21842900 & 1.34985500 & -1.73662900 \\ \text { C } & 1.76016800 & 1.25470600 & 0.32547600 \\ \text { H } & 2.38852500 & 2.14131800 & 0.21130700 \\ \text { H } & 1.29519200 & 1.32807500 & 1.30856000 \\ \text { C } & 1.74350400 & -1.25135000 & 0.35543000 \\ \text { H } & 1.22788000 & -1.28497400 & 1.31816200\end{array}$

$\begin{array}{llll}\mathrm{H} & 2.36038700 & -2.15123100 & 0.29941900\end{array}$

$\begin{array}{llll}\text { C } & 2.61890300 & -0.00488600 & 0.28278700\end{array}$

$\begin{array}{llll}\mathrm{H} & 3.34735800 & 0.00656500 & 1.09616500\end{array}$

H $\quad 3.18568700 \quad-0.02744300-0.65420600$

H $\quad-1.03850289-0.00927133 \quad-0.18593556$

Iso-Butane from 1tBuax
$\begin{array}{lrrr}\mathrm{C} & -1.40990100 & -0.00787100 & 0.09758700 \\ \mathrm{C} & -2.45008800 & 0.94614800 & -0.50136600 \\ \mathrm{H} & -3.33067100 & 0.99861700 & 0.14230600 \\ \mathrm{H} & -2.05534600 & 1.95737900 & -0.60813600 \\ \mathrm{H} & -2.76957400 & 0.59979200 & -1.48631500 \\ \mathrm{C} & -2.04096200 & -1.40001200 & 0.15447300 \\ \mathrm{H} & -3.00661600 & -1.34662000 & 0.66166500 \\ \mathrm{H} & -2.20841400 & -1.79813000 & -0.84895700 \\ \mathrm{H} & -1.41592300 & -2.10556700 & 0.70336000 \\ \mathrm{C} & -1.13607100 & 0.45470000 & 1.53065700 \\ \mathrm{H} & -0.80096400 & 1.49177400 & 1.55957700 \\ \mathrm{H} & -2.06278700 & 0.39352000 & 2.10646100 \\ \mathrm{H} & -0.39337100 & -0.16367500 & 2.03294700 \\ \mathrm{H} & -0.54350611 & -0.01113767 & -0.56381244\end{array}$

Cy from $1 \mathbf{P h}_{\text {eq }} \mathrm{A}$

$\begin{array}{lrrr}\text { C } & 0.34313800 & 0.65509700 & 0.00000000 \\ \text { C } & -0.33420100 & 1.21250300 & -1.25665300 \\ \text { H } & -1.36681000 & 0.84960400 & -1.29205400 \\ \text { H } & 0.16931400 & 0.82394800 & -2.14505000 \\ \text { C } & -0.33420100 & 1.21250300 & 1.25665300 \\ \text { H } & 0.16931400 & 0.82394800 & 2.14505000 \\ \text { H } & -1.36681000 & 0.84960400 & 1.29205400 \\ \text { C } & -0.33420100 & 2.73873800 & 1.25626100 \\ \text { H } & -0.83409300 & 3.11484800 & 2.15124500 \\ \text { H } & 0.69959300 & 3.09725000 & 1.29489700 \\ \text { C } & -0.33420100 & 2.73873800 & -1.25626100 \\ \text { H } & 0.69959300 & 3.09725000 & -1.29489700 \\ \text { H } & -0.83409300 & 3.11484800 & -2.15124500 \\ \text { C } & -1.00645000 & 3.28510300 & 0.00000000 \\ \text { H } & -0.98254900 & 4.37667500 & 0.00000000 \\ \text { H } & -2.06057200 & 2.98893500 & 0.00000000 \\ \text { H } & 1.38274400 & 1.00552400 & 0.00000000 \\ \text { H } & 0.36230915 & -0.43473439 & 0.00000000\end{array}$

$\mathbf{P h}$ from $\mathbf{1 P h}$ eq

$\begin{array}{lrrr}\text { C } & 0.36959400 & -0.84886000 & 0.00000000 \\ \text { C } & 1.58258100 & -1.54364400 & 0.00000000 \\ \text { C } & -0.81808000 & -1.59056000 & 0.00000000 \\ \text { C } & 1.61561200 & -2.93772300 & 0.00000000 \\ \text { H } & 2.51093100 & -0.98307000 & 0.00000000 \\ \text { C } & -0.79260500 & -2.98342800 & 0.00000000 \\ \text { H } & -1.77305800 & -1.07790300 & 0.00000000 \\ \text { C } & 0.42565700 & -3.66237200 & 0.00000000 \\ \text { H } & 2.56658000 & -3.45513200 & 0.00000000 \\ \text { H } & -1.72217000 & -3.53854100 & 0.00000000 \\ \text { H } & 0.44623700 & -4.74435700 & 0.00000000 \\ \text { H } & 0.35059874 & 0.23097294 & 0.00000000\end{array}$

Cy from $1 \mathrm{Ph}_{\mathrm{ax}} \mathrm{B}$

$\begin{array}{llll}\text { C } & -0.70227700 & 0.32783800 & 1.08789900 \\ \text { C } & -1.59280300 & -0.91929600 & 1.10675200\end{array}$ 


$\begin{array}{llll}\mathrm{H} & -2.47183100 & -0.68696000 & 1.71640200 \\ \mathrm{H} & -1.08464400 & -1.74662700 & 1.60709600 \\ \mathrm{C} & -1.45308400 & 1.45909300 & 0.36626300 \\ \mathrm{H} & -0.84850000 & 2.36621100 & 0.33897500 \\ \mathrm{H} & -2.35093300 & 1.69800600 & 0.94600000 \\ \mathrm{C} & -1.86601000 & 1.04788900 & -1.04316100 \\ \mathrm{H} & -2.37214100 & 1.87731100 & -1.54134900 \\ \mathrm{H} & -0.97089300 & 0.81792600 & -1.62986100 \\ \mathrm{C} & -2.06825600 & -1.33370800 & -0.28689100 \\ \mathrm{H} & -1.21825900 & -1.64701300 & -0.89707500 \\ \mathrm{H} & -2.73604200 & -2.19424900 & -0.20512700 \\ \mathrm{C} & -2.77204400 & -0.17882500 & -0.99411900 \\ \mathrm{H} & -3.06846800 & -0.47818400 & -2.00137900 \\ \mathrm{H} & -3.68924500 & 0.07554900 & -0.45244200 \\ \mathrm{H} & -0.54831000 & 0.64254300 & 2.12628500 \\ \mathrm{H} & 0.28862951 & 0.16522961 & 0.66391457\end{array}$

Ph from $1 \mathbf{P h}_{\mathrm{ax}}$ B

$\begin{array}{lrrr}\text { C } & 0.67884500 & 0.10119500 & 0.49695100 \\ \text { C } & 1.16906000 & -1.16694700 & 0.16585200 \\ \text { C } & 1.53936700 & 1.19342500 & 0.31765700 \\ \text { C } & 2.45711900 & -1.33666800 & -0.34363000 \\ \text { H } & 0.55059800 & -2.04229400 & 0.30833500 \\ \text { C } & 2.82636700 & 1.03211100 & -0.18910400 \\ \text { H } & 1.20681900 & 2.18764100 & 0.58986600 \\ \text { C } & 3.29058700 & -0.23742300 & -0.52981400 \\ \text { H } & 2.80565300 & -2.33101800 & -0.59297300 \\ \text { H } & 3.46642400 & 1.89650000 & -0.31309100 \\ \text { H } & 4.28853900 & -0.36744600 & -0.92758600 \\ \text { H } & -0.30297063 & 0.26231157 & 0.91704566\end{array}$

Cy from $1 \mathrm{Ph}_{\mathrm{ax}} \mathrm{C}$

$\begin{array}{llrr}\text { C } & 1.03794200 & -0.68321800 & 0.00000000 \\ \text { C } & 1.65128600 & -0.05410900 & 1.26349200 \\ \text { H } & 2.69176900 & -0.39145300 & 1.32619900 \\ \text { H } & 1.13710000 & -0.43908700 & 2.14703600 \\ \text { C } & 1.65128600 & -0.05410900 & -1.26349200 \\ \text { H } & 1.13710000 & -0.43908700 & -2.14703600 \\ \text { H } & 2.69176900 & -0.39145300 & -1.32619900 \\ \text { C } & 1.65128600 & 1.47255900 & -1.26127500 \\ \text { H } & 2.17703600 & 1.83371800 & -2.14796800 \\ \text { H } & 0.63010900 & 1.85115300 & -1.33595300 \\ \text { C } & 1.65128600 & 1.47255900 & 1.26127500 \\ \text { H } & 0.63010900 & 1.85115300 & 1.33595300 \\ \text { H } & 2.17703600 & 1.83371800 & 2.14796800 \\ \text { C } & 2.31871400 & 2.01560400 & 0.00000000 \\ \text { H } & 2.30235200 & 3.10738100 & 0.00000000 \\ \text { H } & 3.37168000 & 1.71368700 & 0.00000000 \\ \text { H } & 1.34074800 & -1.73467700 & 0.00000000 \\ \text { H } & -0.05187823 & -0.70301339 & 0.00000000\end{array}$

$\mathbf{P h}$ from $\mathbf{1} \mathbf{P h}_{\mathrm{ax}} \mathbf{C}$

$\begin{array}{llrl}\text { C } & -0.47258100 & -0.71065500 & 0.00000000 \\ \text { C } & -1.28964500 & 0.43005500 & 0.00000000 \\ \text { C } & -1.11171200 & -1.95857100 & 0.00000000 \\ \text { C } & -2.68025800 & 0.32154100 & 0.00000000 \\ \text { H } & -0.85363300 & 1.41704800 & 0.00000000 \\ \text { C } & -2.50010900 & -2.07431000 & 0.00000000 \\ \text { H } & -0.50389900 & -2.85664200 & 0.00000000 \\ \text { C } & -3.29327400 & -0.92922800 & 0.00000000\end{array}$

$\begin{array}{lrrr}H & -3.28358500 & 1.22071500 & 0.00000000 \\ H & -2.95859600 & -3.05506200 & 0.00000000 \\ H & -4.37241500 & -1.00982300 & 0.00000000 \\ H & 0.60724088 & -0.69104122 & 0.00000000\end{array}$

$\begin{array}{lrrr}\text { Cy from 1CF } & & & \\ \mathrm{C} & 0.39022700 & 0.00156800 & 0.00000000 \\ \mathrm{C} & -0.28974500 & -0.53285600 & 1.25979200 \\ \mathrm{H} & -1.32028900 & -0.16811400 & 1.28501500 \\ \mathrm{H} & 0.21347300 & -0.15127700 & 2.14868100 \\ \mathrm{C} & -0.28974500 & -0.53285600 & -1.25979200 \\ \mathrm{H} & 0.21347300 & -0.15127700 & -2.14868100 \\ \mathrm{H} & -1.32028900 & -0.16811400 & -1.28501500 \\ \mathrm{C} & -0.28974500 & -2.05905400 & -1.25571800 \\ \mathrm{H} & -0.79188400 & -2.43022300 & -2.15055800 \\ \mathrm{H} & 0.74307400 & -2.41901000 & -1.29800700 \\ \mathrm{C} & -0.28974500 & -2.05905400 & 1.25571800 \\ \mathrm{H} & 0.74307400 & -2.41901000 & 1.29800700 \\ \mathrm{H} & -0.79188400 & -2.43022300 & 2.15055800 \\ \mathrm{C} & -0.96167100 & -2.60603700 & 0.00000000 \\ \mathrm{H} & -0.93433700 & -3.69705900 & 0.00000000 \\ \mathrm{H} & -2.01601500 & -2.31229500 & 0.00000000 \\ \mathrm{H} & 1.43962900 & -0.31006400 & 0.00000000 \\ \mathrm{H} & 0.41182019 & 1.09135410 & 0.00000000\end{array}$

$\begin{array}{lrrr}\mathbf{H C F}_{3} \text { from } & \mathbf{1 C F}_{\mathbf{3 e q}} & & \\ \mathrm{C} & 0.42007700 & 1.50806700 & 0.00000000 \\ \mathrm{~F} & 1.05754400 & 1.99774400 & 1.08112500 \\ \mathrm{~F} & 1.05754400 & 1.99774400 & -1.08112500 \\ \mathrm{~F} & -0.81685900 & 2.04206700 & 0.00000000 \\ \mathrm{H} & 0.39848381 & 0.41828090 & 0.00000000\end{array}$

\begin{tabular}{|c|c|c|c|}
\hline \multicolumn{4}{|c|}{ Cy from $\mathbf{1 C F}_{3 a x}$} \\
\hline C & -0.92532500 & -0.02098000 & 0.00000000 \\
\hline $\mathrm{H}$ & -1.98972300 & 0.22772200 & 0.00000000 \\
\hline C & -0.61618100 & -0.82626600 & 1.26731600 \\
\hline $\mathrm{H}$ & -1.36877900 & -1.61728900 & 1.32942000 \\
\hline $\mathrm{H}$ & -0.74637100 & -0.19823200 & 2.14934900 \\
\hline C & -0.61618100 & -0.82626600 & -1.26731600 \\
\hline $\mathrm{H}$ & -0.74637100 & -0.19823200 & -2.14934900 \\
\hline $\mathrm{H}$ & -1.36877900 & -1.61728900 & -1.32942000 \\
\hline C & 0.76709100 & -1.47518300 & -1.25668000 \\
\hline $\mathrm{H}$ & 0.87510900 & -2.09506600 & -2.14858500 \\
\hline $\mathrm{H}$ & 1.54225000 & -0.70903000 & -1.30626600 \\
\hline C & 0.76709100 & -1.47518300 & 1.25668000 \\
\hline-1 & 1.54225000 & -0.70903000 & 1.30626600 \\
\hline H & 0.87510900 & -2.09506600 & 2.14858500 \\
\hline C & 0.96359000 & -2.31805000 & 0.00000000 \\
\hline † & 1.95583300 & -2.77231800 & 0.00000000 \\
\hline$H$ & 0.23723700 & -3.13784300 & 0.00000000 \\
\hline $\mathrm{H}$ & -0.44430122 & 0.95713867 & 0.00000000 \\
\hline
\end{tabular}




$\begin{array}{lrrr}\text { Cy from } 1 \mathrm{CCl}_{3 \text { eq }} & & \\ \mathrm{C} & -0.41201000 & 0.40821700 & 0.00000000 \\ \mathrm{C} & 0.03013700 & 1.16530300 & 1.25282100 \\ \mathrm{H} & 1.12205800 & 1.22164800 & 1.26348800 \\ \mathrm{H} & -0.27928400 & 0.62877100 & 2.14920700 \\ \mathrm{C} & 0.03013700 & 1.16530300 & -1.25282100 \\ \mathrm{H} & -0.27928400 & 0.62877100 & -2.14920700 \\ \mathrm{H} & 1.12205800 & 1.22164800 & -1.26348800 \\ \mathrm{C} & -0.55346100 & 2.57687900 & -1.25251300 \\ \mathrm{H} & -0.22723200 & 3.10578400 & -2.14970700 \\ \mathrm{H} & -1.64529400 & 2.51511400 & -1.29894800 \\ \mathrm{C} & -0.55346100 & 2.57687900 & 1.25251300 \\ \mathrm{H} & -1.64529400 & 2.51511400 & 1.29894800 \\ \mathrm{H} & -0.22723200 & 3.10578400 & 2.14970700 \\ \mathrm{C} & -0.14409800 & 3.34324300 & 0.00000000 \\ \mathrm{H} & -0.59005500 & 4.33948600 & 0.00000000 \\ \mathrm{H} & 0.94207500 & 3.47765700 & 0.00000000 \\ \mathrm{H} & -1.50618000 & 0.33574300 & 0.00000000 \\ \mathrm{H} & -0.07302960 & -0.62773295 & 0.00000000\end{array}$

$\mathrm{HCCl}_{3}$ from $1 \mathrm{CCl}_{3 e q}$

$\begin{array}{llll}\text { C } & 0.06309300 & -1.04373400 & 0.00000000\end{array}$

Cl $\quad-0.55346100-1.90301100 \quad 1.43550000$

Cl $\quad \quad-0.55346100-1.90301100 \quad-1.43550000$

$\begin{array}{lllll}\text { Cl } & 1.83937100 & -1.14974500 & 0.00000000\end{array}$

$\begin{array}{llll}\text { H } & -0.27588740 & -0.00778405 & 0.00000000\end{array}$

Cy from $\mathbf{1 C C l}_{3 \mathrm{ax}}$

$\begin{array}{llll}\text { C } & -0.89353400 & -0.43069000 & 0.00000000 \\ \text { H } & -1.94343800 & -0.12105400 & 0.00000000 \\ \text { C } & -0.66701000 & -1.27363800 & 1.26584400 \\ \text { H } & -1.53524300 & -1.93604500 & 1.34027200 \\ \text { H } & -0.68792100 & -0.63649500 & 2.14952300 \\ \text { C } & -0.66701000 & -1.27363800 & -1.26584400 \\ \text { H } & -0.68792100 & -0.63649500 & -2.14952300 \\ \text { H } & -1.53524300 & -1.93604500 & -1.34027200 \\ \text { C } & 0.58707300 & -2.14651200 & -1.25277700 \\ \text { H } & 0.58077500 & -2.77326400 & -2.14698100 \\ \text { H } & 1.48307900 & -1.52815700 & -1.30614500 \\ \text { C } & 0.58707300 & -2.14651200 & 1.25277700 \\ \text { H } & 1.48307900 & -1.52815700 & 1.30614500 \\ \text { H } & 0.58077500 & -2.77326400 & 2.14698100 \\ \text { C } & 0.63710800 & -3.01355100 & 0.00000000 \\ \text { H } & 1.53702700 & -3.63106500 & 0.00000000 \\ \text { H } & -0.21949000 & -3.69644100 & 0.00000000 \\ \text { H } & -0.38351593 & 0.53262800 & 0.00000000\end{array}$

$\mathrm{HCCl}_{3}$ from $\mathbf{1 C C l}_{3 \mathrm{ax}}$

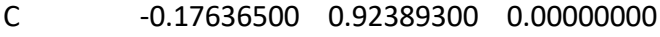

Cl $\quad-\quad-0.66701000 \quad 1.86718900 \quad-1.43531100$

$\begin{array}{llll}\text { Cl } & -0.66701000 & 1.86718900 & 1.43531100\end{array}$

$\begin{array}{lllll}\text { Cl } & & 1.59875600 & 0.81623100 & 0.00000000\end{array}$

$\begin{array}{llll}\mathrm{H} & -0.68638307 & -0.03942500 & 0.00000000\end{array}$

Cy from $1 \mathrm{CBr}_{3 \mathrm{eq}}$

$\begin{array}{llll}\text { C } & -1.03102400 & 0.00000000 & 0.44669200\end{array}$

C $\quad-1.76835600 \quad 1.25056300 \quad-0.03258100$

H $\quad-1.77423500 \quad 1.25586700 \quad-1.12602500$ $\begin{array}{lll}-1.24434500 & 2.14699100 & 0.29704500\end{array}$

$-1.76835500-1.25056400-0.03258100$

$\begin{array}{lll}-1.24434400 & -2.14699100 & 0.29704600\end{array}$

$-1.77423400-1.25586800-1.12602500$

$\begin{array}{lll}-3.20384600 & -1.25230200 & 0.48798300\end{array}$

$\begin{array}{lll}-3.71683800 & -2.14949300 & 0.13716800\end{array}$

$\begin{array}{lll}-3.19030600 & -1.30016100 & 1.58138100\end{array}$

$\begin{array}{lll}-3.20384700 & 1.25230100 & 0.48798200\end{array}$

$\begin{array}{lll}-3.19030800 & 1.30016000 & 1.58138000\end{array}$

$\begin{array}{lll}-3.71684000 & 2.14949100 & 0.13716600\end{array}$

$\begin{array}{lll}-3.95194800 & -0.00000100 & 0.04597900\end{array}$

$\begin{array}{lll}-4.96658900 & -0.00000100 & 0.44836000\end{array}$

$\begin{array}{lll}-4.03864900 & -0.00000200 & -1.04499200\end{array}$

$\begin{array}{lll}-1.01610500 & 0.00000000 & 1.54383900\end{array}$

$\begin{array}{lll}0.02116410 & 0.00000000 & 0.16208665\end{array}$

$\begin{array}{lrrr}\text { Cy from } 1 \mathrm{CBr}_{3 \mathrm{ax}} & & \\ \mathrm{C} & -0.94618588 & -0.97722837 & 0.00000000 \\ \mathrm{H} & -1.99156388 & -0.65010437 & 0.00000000 \\ \mathrm{C} & -0.74044488 & -1.82633037 & 1.26606500 \\ \mathrm{H} & -1.63222288 & -2.45583537 & 1.34849200 \\ \mathrm{H} & -0.73277188 & -1.18481637 & 2.14664700 \\ \mathrm{C} & -0.74044488 & -1.82633037 & -1.26606500 \\ \mathrm{H} & -0.73277188 & -1.18481637 & -2.14664700 \\ \mathrm{H} & -1.63222288 & -2.45583537 & -1.34849200 \\ \mathrm{C} & 0.48033012 & -2.74430837 & -1.25119600 \\ \mathrm{H} & 0.45327912 & -3.36826537 & -2.14696900 \\ \mathrm{H} & 1.39745712 & -2.15797037 & -1.30020400 \\ \mathrm{C} & 0.48033012 & -2.74430837 & 1.25119600 \\ \mathrm{H} & 1.39745712 & -2.15797037 & 1.30020400 \\ \mathrm{H} & 0.45327912 & -3.36826537 & 2.14696900 \\ \mathrm{C} & 0.49583812 & -3.61413637 & 0.00000000 \\ \mathrm{H} & 1.37089712 & -4.26642537 & 0.00000000 \\ \mathrm{H} & -0.38699988 & -4.26276337 & 0.00000000 \\ \mathrm{H} & -0.42212647 & -0.02147645 & 0.00000000\end{array}$

$\mathrm{HCBr}_{3}$ from $1 \mathrm{CBr}_{3 a x}$

$\begin{array}{llll}\text { C } & -0.20555400 & 0.37349700 & 0.00000000\end{array}$

$\mathrm{Br} \quad-0.73563700 \quad 1.40805100 \quad-1.55881300$

$\begin{array}{llll}\mathrm{Br} & -0.73563700 & 1.40805100 & 1.55881300\end{array}$

$\begin{array}{lllll}\mathrm{Br} & 1.72461700 & 0.24835100 & 0.00000000\end{array}$

$\begin{array}{llll}\text { H } & -0.72961341 & -0.58225492 & 0.00000000\end{array}$

Cy from $\mathbf{1 C l}_{3 \text { eq }}$

$\begin{array}{llll}\text { C } & 0.58555700 & -1.33346700 & 0.00000000 \\ \text { C } & 0.16872500 & -2.11388100 & 1.24733500 \\ \text { H } & -0.92068700 & -2.21393600 & 1.24968900 \\ \text { H } & 0.44819600 & -1.56587500 & 2.14708600 \\ \text { C } & 0.16872500 & -2.11388100 & -1.24733500 \\ \text { H } & 0.44819600 & -1.56587500 & -2.14708600 \\ \text { H } & -0.92068700 & -2.21393600 & -1.24968900 \\ \text { C } & 0.81130300 & -3.49933900 & -1.25172400\end{array}$




\begin{abstract}
$0.50391000-4.03854300-2.14956400$
$\begin{array}{lll}0.89934200 & -3.39107500 & -1.30144900\end{array}$

$\begin{array}{llll}0.81130300 & -3.49933900 & 1.25172400\end{array}$

$\begin{array}{lll}1.89934200 & -3.39107500 & 1.30144900\end{array}$

$\begin{array}{llll}0.50391000 & -4.03854300 & 2.14956400\end{array}$

$\begin{array}{llll}0.43645300 & -4.28359200 & 0.00000000\end{array}$

$\begin{array}{lll}0.92581000 & -5.25933400 & 0.00000000\end{array}$

$\begin{array}{lll}-0.64288900 & -4.46464000 & 0.00000000\end{array}$

$\begin{array}{lll}1.67992800 & -1.23709000 & 0.00000000\end{array}$

$\begin{array}{lll}0.22089211 & -0.30627689 & 0.00000000\end{array}$
\end{abstract}

$\mathrm{HCl}_{3}$ from $1 \mathrm{Cl}_{3 \mathrm{eq}}$

$\begin{array}{crrr}\text { C } & 0.07382200 & 0.10799100 & 0.00000000 \\ \text { I } & 0.81130300 & 1.16649300 & 1.73163000 \\ \text { I } & 0.81130300 & 1.16649300 & -1.73163000 \\ \text { I } & -2.07844900 & 0.19141000 & 0.00000000 \\ \text { H } & 0.43848689 & -0.91919911 & 0.00000000\end{array}$

Cy from $\mathbf{1 C l}_{3 \mathbf{a x}}$

$\begin{array}{lrrr}\text { C } & -1.29353700 & -0.96981200 & 0.00000000 \\ \text { H } & -2.20751600 & -0.36398800 & 0.00000000 \\ \text { C } & -1.34720100 & -1.84621700 & 1.26551600 \\ \text { H } & -2.38997900 & -2.16708500 & 1.35874400 \\ \text { H } & -1.13381100 & -1.24022500 & 2.14618600 \\ \text { C } & -1.34720100 & -1.84621700 & -1.26551600 \\ \text { H } & -1.13381100 & -1.24022500 & -2.14618600 \\ \text { H } & -2.38997900 & -2.16708500 & -1.35874400 \\ \text { C } & -0.47348500 & -3.09877300 & -1.24858700 \\ \text { H } & -0.69052200 & -3.68025100 & -2.14723400 \\ \text { H } & 0.58136000 & -2.82849300 & -1.29353100 \\ \text { C } & -0.47348500 & -3.09877300 & 1.24858700 \\ \text { H } & 0.58136000 & -2.82849300 & 1.29353100 \\ \text { H } & -0.69052200 & -3.68025100 & 2.14723400 \\ \text { C } & -0.73306200 & -3.93178900 & 0.00000000 \\ \text { H } & -0.10584700 & -4.82507900 & 0.00000000 \\ \text { H } & -1.77477600 & -4.27101300 & 0.00000000 \\ \text { H } & -0.52475567 & -0.19710554 & 0.00000000\end{array}$

$\mathrm{HCl}_{3}$ from $\mathrm{1Cl}_{3 \mathrm{ax}}$

$\begin{array}{llll}\text { C } & -0.21022700 & 0.11902900 & 0.00000000\end{array}$

l $\quad-0.47348500 \quad 1.39533200 \quad-1.72996500$

$\begin{array}{lllll}\text { I } & -0.47348500 & 1.39533200 & 1.72996500\end{array}$

$\begin{array}{lllll}\text { I } & 1.82665300 & -0.57693700 & 0.00000000\end{array}$

H $\quad-0.97900833 \quad-0.65367746 \quad 0.00000000$

\begin{tabular}{lrrr}
\multicolumn{4}{l}{ Cy from $1 \mathrm{SiMe}_{3 \mathrm{eq}}$} \\
$\mathrm{C}$ & -0.44277300 & 0.21866900 & 0.00000000 \\
$\mathrm{C}$ & 0.00300800 & 0.97715300 & 1.25753100 \\
$\mathrm{H}$ & 1.09858400 & 0.98588000 & 1.30145500 \\
$\mathrm{H}$ & -0.34398800 & 0.46261400 & 2.15738900 \\
$\mathrm{C}$ & 0.00300800 & 0.97715300 & -1.25753100 \\
$\mathrm{H}$ & -0.34398800 & 0.46261400 & -2.15738900 \\
$\mathrm{H}$ & 1.09858400 & 0.98588000 & -1.30145500 \\
$\mathrm{C}$ & -0.49881000 & 2.42021400 & -1.25671800 \\
$\mathrm{H}$ & -0.15037300 & 2.94284300 & -2.15065700 \\
$\mathrm{H}$ & -1.59303200 & 2.41562600 & -1.29398100 \\
$\mathrm{C}$ & -0.49881000 & 2.42021400 & 1.25671800 \\
$\mathrm{H}$ & -1.59303200 & 2.41562600 & 1.29398100 \\
$\mathrm{H}$ & -0.15037300 & 2.94284300 & 2.15065700
\end{tabular}

$\begin{array}{lrrr}\mathrm{C} & -0.04356000 & 3.15603300 & 0.00000000 \\ \mathrm{H} & -0.42237300 & 4.18022100 & 0.00000000 \\ \mathrm{H} & 1.04967700 & 3.22096100 & 0.00000000 \\ \mathrm{H} & -1.54249700 & 0.18703700 & 0.00000000 \\ \mathrm{H} & -0.10261168 & -0.81689378 & 0.00000000\end{array}$

$\begin{array}{lrrr}\mathrm{HSiMe}_{3} & \text { from 1SiMe } \\ \mathrm{Si} & 0.14845000 & -1.58120800 & 0.00000000 \\ \mathrm{C} & -0.49881000 & -2.45745900 & 1.53721600 \\ \mathrm{H} & -0.21964600 & -3.51240400 & 1.52593500 \\ \mathrm{H} & -0.09130800 & -2.01467300 & 2.44684600 \\ \mathrm{H} & -1.58717900 & -2.39926300 & 1.59192300 \\ \mathrm{C} & -0.49881000 & -2.45745900 & -1.53721600 \\ \mathrm{H} & -0.09130800 & -2.01467300 & -2.44684600 \\ \mathrm{H} & -0.21964600 & -3.51240400 & -1.52593500 \\ \mathrm{H} & -1.58717900 & -2.39926300 & -1.59192300 \\ \mathrm{C} & 2.03237800 & -1.63237900 & 0.00000000 \\ \mathrm{H} & 2.44202600 & -1.14017100 & 0.88313100 \\ \mathrm{H} & 2.38580300 & -2.66504200 & 0.00000000 \\ \mathrm{H} & 2.44202600 & -1.14017100 & -0.88313100 \\ \mathrm{H} & -0.31342042 & -0.17512275 & 0.00000000\end{array}$

Cy from 1SiMe 3 ax

$\begin{array}{llll}\text { C } & 0.19778000 & 0.00112300 & -1.01296000 \\ \text { H } & -0.24756500 & -0.00090200 & -2.01810600 \\ \text { C } & 1.07860400 & -1.25071100 & -0.90115200 \\ \text { H } & 1.76876900 & -1.27611000 & -1.75354100 \\ \text { H } & 0.47094600 & -2.15620600 & -0.96496900 \\ \text { C } & 1.06478500 & 1.26495300 & -0.89711400 \\ \text { H } & 0.44348300 & 2.16513400 & -0.92146800 \\ \text { H } & 1.72495200 & 1.32090900 & -1.77113400 \\ \text { C } & 1.93531400 & 1.26351700 & 0.35961000 \\ \text { H } & 2.57805600 & 2.14681900 & 0.36229800 \\ \text { H } & 1.30401700 & 1.32978600 & 1.24782800 \\ \text { C } & 1.90669700 & -1.25192400 & 0.38046500 \\ \text { H } & 1.24173400 & -1.27811500 & 1.24965800 \\ \text { H } & 2.52464200 & -2.15127600 & 0.42802100 \\ \text { C } & 2.78197100 & -0.00339800 & 0.44869900 \\ \text { H } & 3.37322000 & 0.00134400 & 1.36682300 \\ \text { H } & 3.48987800 & -0.02357000 & -0.38666700 \\ \text { H } & -0.69479624 & -0.00214601 & -0.38734273\end{array}$

$\begin{array}{lrrr}\mathrm{HSiMe}_{3} & \text { from 1SiMe } \\ \text { Si } & & & \\ \mathrm{Si} & -1.36210800 & -0.00459000 & 0.08038400 \\ \mathrm{C} & -2.58674100 & 1.19130800 & -0.70994600 \\ \mathrm{H} & -3.51125500 & 1.24092600 & -0.13232600 \\ \mathrm{H} & -2.17258200 & 2.19973400 & -0.76163500 \\ \mathrm{H} & -2.83926200 & 0.88021300 & -1.72477500 \\ \mathrm{C} & -2.08810000 & -1.74360000 & 0.07294400 \\ \mathrm{H} & -3.06320800 & -1.75254300 & 0.56305200 \\ \mathrm{H} & -2.21995000 & -2.11250300 & -0.94565600 \\ \mathrm{H} & -1.44114400 & -2.44428200 & 0.60313800 \\ \mathrm{C} & -1.13108900 & 0.53412900 & 1.87456200 \\ \mathrm{H} & -0.81263900 & 1.57500300 & 1.94308200 \\ \mathrm{H} & -2.09039200 & 0.44795700 & 2.39042100 \\ \mathrm{H} & -0.40750700 & -0.08043500 & 2.40993200 \\ \mathrm{H} & -0.15016962 & -0.00015135 & -0.76907798\end{array}$

Bimolecular complexes: 
Cy $\cdots F_{2}$ (MP2/aug-cc-pVTZ)

Imaginary frequencies- 0

Electronic Energy (SCF) $=-433.041371677$ a.u

Electronic Energy (MP2) $=-434.6398595577$ a.u

ZPV corrected Energy $=-434.465475$ a.u.

Enthalpy $=-434.454487$ a.u.

Free Energy = -434.504046 a.u.

C $\quad-1.258667-0.726692-0.888868$

C $0.000000 \quad-1.453084-1.357275$

H $\quad 0.000000 \quad-1.498036-2.451341$

H $\quad 0.000000 \quad-2.483876-0.997100$

C $\quad-1.258407 \quad 0.726542 \quad-1.357275$

H $\quad-2.151100 \quad 1.241938-0.997100$

H $\quad-1.297337 \quad 0.749018-2.451341$

C $0.000000 \quad 1.453383 \quad-0.888868$

H $\quad-0.000000 \quad 2.483776-1.250225$

$\begin{array}{llll}H & -0.000000 & 1.502224 & 0.204801\end{array}$

C $1.258667 \quad-0.726692-0.888868$

$\begin{array}{llll}\text { H } & 1.300964 & -0.751112 & 0.204801\end{array}$

H $\quad 2.151013 \quad-1.241888-1.250225$

C $\quad 1.258407 \quad 0.726542 \quad-1.357275$

H $\quad 2.151100 \quad 1.241938-0.997100$

H $\quad \begin{array}{llll}1.297337 & 0.749018 & -2.451341\end{array}$

H $\quad-1.300964 \quad-0.751112 \quad 0.204801$

H $\quad-2.151013-1.241888-1.250225$

$\begin{array}{llll}F & 0.000000 & 0.000000 & 2.292933\end{array}$

F $\quad 0.000000 \quad 0.000000 \quad 3.697309$

\section{$\mathrm{Cy} \cdot \cdots \mathrm{Cl}_{2}$ (MP2/aug-cc-pVTZ)}

Imaginary frequencies- 0

Electronic Energy (SCF) $=-1153.28340944$ a.u Electronic Energy (MP2) $=-1154.7381557257$ a.u ZPV corrected Energy $=-1154.564828$ a.u. Enthalpy $=-1154.553718$ a.u.

Free Energy = -1154.604186 a.u.

$\begin{array}{llll}\text { C } & -1.258947 & -0.726853 & -1.703717\end{array}$

C $-0.000000-1.453583-2.170393$

H $\quad-0.000000-1.499911-3.264437$

H $\quad-0.000000-2.483875-1.808809$

C $-1.258840 \quad 0.726792 \quad-2.170393$

H $\quad-2.151099 \quad 1.241937 \quad-1.808809$

H $\quad-1.298961 \quad 0.749956-3.264437$

C $0.000000 \quad 1.453706-1.703717$

H $\quad 0.000000 \quad 2.483962-2.065523$

H $\quad 0.000000 \quad 1.506696-0.609569$

$\begin{array}{llll}\text { C } & 1.258947 & -0.726853 & -1.703717\end{array}$

H $\quad 1.304837 \quad-0.753348 \quad-0.609569$

H $\quad 2.151174 \quad-1.241981-2.065523$

C $\quad 1.258840 \quad 0.726792 \quad-2.170393$

H $\quad 2.151099 \quad 1.241937 \quad-1.808809$

H $\quad 1.298961 \quad 0.749956 \quad-3.264437$

H $\quad-1.304837-0.753348-0.609569$

H $\quad-2.151174-1.241981-2.065523$

$\begin{array}{llll}\text { Cl } & 0.000000 & 0.000000 & 3.735932\end{array}$

$\begin{array}{llll}\mathrm{Cl} & 0.000000 & 0.000000 & 1.733420\end{array}$

$\mathrm{Cy} \cdot \cdots \mathrm{Br}_{2}$ (MP2/aug-cc-pVTZ)

Imaginary frequencies- 0
Electronic Energy (SCF) $=-5379.19626913$ a.u Electronic Energy (MP2) $=-5380.7337127600 \mathrm{a} . \mathrm{u}$ ZPV corrected Energy = -5380.560933 a.u. Enthalpy $=-5380.549649$ a.u.

Free Energy $=-5380.601709$ a.u.

C $\quad-1.259004-0.726886-2.587938$

C $0.000000 \quad-1.454043 \quad-3.053223$

H $\quad 0.000000-1.502295 \quad-4.147192$

H $\quad 0.000000 \quad-2.483626-2.689620$

C $-1.259238 \quad 0.727021 \quad-3.053223$

H $\quad-2.150883 \quad 1.241813 \quad-2.689620$

H $\quad-1.301025 \quad 0.751147 \quad-4.147192$

C $0.000000 \quad 1.453772 \quad-2.587938$

H $\quad-0.000000 \quad 2.484257 \quad-2.948869$

H $\quad-0.000000 \quad 1.508226-1.493496$

C $1.259004-0.726886-2.587938$

H $\quad 1.306162-0.754113-1.493496$

H $\quad 2.151430 \quad-1.242129-2.948869$

C $1.259238 \quad 0.727021 \quad-3.053223$

H $2.150883 \quad 1.241813 \quad-2.689620$

H $\quad 1.301025 \quad 0.751147 \quad-4.147192$

H $\quad-1.306162-0.754113-1.493496$

H $\quad-2.151430-1.242129 \quad-2.948869$

$\begin{array}{llll}\mathrm{Br} & 0.000000 & 0.000000 & 0.791390\end{array}$

$\begin{array}{llll}\mathrm{Br} & 0.000000 & 0.000000 & 3.076565\end{array}$

\section{Cy $\cdots I_{2}$ (MP2/aug-cc-pVTZ)}

Imaginary frequencies- 0

Electronic Energy (SCF) $=-823.624643007$ a.u Electronic Energy (MP2) $=-825.1253211218$ a.u ZPV corrected Energy $=-824.952899$ a.u. Enthalpy $=-824.941478$ a.u.

Free Energy $=-824.994496$ a.u.

$\begin{array}{llll}\text { C } & -1.258851 & -0.726798 & -3.264692\end{array}$

C $0.000000 \quad-1.454345-3.729128$

H $\quad 0.000000-1.503406-4.823073$

H $\quad 0.000000 \quad-2.483657 \quad-3.364761$

C $\quad-1.259499 \quad 0.727172 \quad-3.729128$

H $\quad-2.150910 \quad 1.241828-3.364761$

H $\quad-1.301987 \quad 0.751703-4.823073$

C $0.000000 \quad 1.453596 \quad-3.264692$

H $\quad-0.000000 \quad 2.484674 \quad-3.623881$

H $\quad-0.000000 \quad 1.508032-2.169608$

C $1.258851-0.726798-3.264692$

H $1.305994-0.754016 \quad-2.169608$

H $\quad 2.151790 \quad-1.242337-3.623881$

C $1.259499 \quad 0.727172 \quad-3.729128$

H $\quad 2.150910 \quad 1.241828-3.364761$

H $\quad 1.301987 \quad 0.751703 \quad-4.823073$

H $\quad-1.305994-0.754016-2.169608$

H $\quad-2.151790 \quad-1.242337 \quad-3.623881$

I $\quad 0.000000 \quad 0.000000 \quad 2.918541$

\begin{tabular}{llll}
\hline & 0.000000 & 0.000000 & 0.248114
\end{tabular}

\section{Cy... $\mathrm{CH}_{4}$ (MP2/aug-cc-pVTZ)}

Imaginary frequencies- 0

Electronic Energy (SCF) $=-274.499096262$ a.u Electronic Energy (MP2) $=-275.7632971635$ a.u ZPV corrected Energy = -275.545466 a.u. 
Enthalpy $=-275.533895$ a.u.

Free Energy $=-275.582672$ a.u.

C $0.000000 \quad 1.452990 \quad-0.466440$

C $-1.258209 \quad 0.726428-0.936175$

H $\quad-1.296871 \quad 0.748749 \quad-2.030327$

H $\quad-2.151195 \quad 1.241993 \quad-0.576464$

C $1.2582090 .726428 \quad-0.936175$

H $2.151195 \quad 1.241993 \quad-0.576464$

H $\quad 1.296871 \quad 0.748749-2.030327$

C $1.258326-0.726495-0.466440$

H $\quad 2.151173-1.241980-0.826418$

H $\quad 1.296129-0.748320 \quad 0.627275$

C $-1.258326-0.726495-0.466440$

H $-1.296129-0.748320 \quad 0.627275$

H $\quad-2.151173-1.241980-0.826418$

C $-0.000000-1.452855-0.936175$

H $\quad-0.000000-2.483986-0.576464$

H $\quad-0.000000-1.497498-2.030327$

H $\quad 0.000000 \quad 1.496641 \quad 0.627275$

$\begin{array}{llll}\text { H } & 0.000000 & 2.483960 & -0.826418\end{array}$

C $-0.000000 \quad 0.000000 \quad 3.366750$

H $\quad-0.000000 \quad 0.000000 \quad 4.453267$

H $\quad 0.887025 \quad 0.512124 \quad 3.003707$

$\begin{array}{llll}\mathrm{H} & -0.000000 & -1.024248 & 3.003707\end{array}$

H $\quad-0.887025 \quad 0.512124 \quad 3.003707$

Geometries for the $2 \mathrm{H}+3 \mathrm{R} \rightarrow 2 \mathrm{R}+3 \mathrm{H}$, optimized at B3LYP-D3(BJ)/def2-TZVPP:

\section{H (B3LYP-D3(BJ)/def2-TZVPP)}

Imaginary frequencies- 0

Electronic Energy (SCF) $=-390.919371363$ a.u

Grimme-D3(BJ) Dispersion energy $=-0.0527118573$ a.u

ZPV corrected Energy = -390.675813 a.u.

Enthalpy $=-390.667912$ a.u.

Free Energy $=-390.704247$ a.u.

$\begin{array}{llll}C & 0.888263 & 0.888263 & 0.888263\end{array}$

H $\quad 1.519068 \quad 1.5190681 .519068$

C $\quad-0.000000 \quad 0.000000 \quad 1.772446$

H $\quad 0.621722 \quad-0.621722 \quad 2.422325$

H $\quad-0.621722 \quad 0.621722 \quad 2.422325$

C $0.000000 \quad 1.772446-0.000000$

H $\quad-0.621722 \quad 2.422325 \quad 0.621722$

H $\quad 0.621722 \quad 2.422325 \quad-0.621722$

C $1.772446 \quad 0.000000 \quad 0.000000$

H $\quad 2.422325 \quad 0.621722 \quad-0.621722$

H $\quad 2.422325 \quad-0.6217220 .621722$

C $-0.888263-0.8882630 .888263$

H $\quad-1.519068$-1.519068 1.519068

C $\quad-0.888263 \quad 0.888263 \quad-0.888263$

H $\quad-1.519068 \quad 1.519068-1.519068$

C $0.888263-0.888263-0.888263$

H $\quad 1.519068-1.519068-1.519068$

C $-0.000000-1.7724460 .000000$

$\begin{array}{llll}\text { H } & 0.621722 & -2.422325 & 0.621722\end{array}$

H $\quad-0.621722-2.422325 \quad-0.621722$

C $-0.000000-0.000000-1.772446$

H $\quad 0.6217220 .621722-2.422325$

H $\quad-0.621722 \quad-0.621722-2.422325$

C $-1.772446 \quad 0.000000-0.000000$ $\begin{array}{llll}\text { H } & -2.422325 & -0.621722 & -0.621722\end{array}$

$\begin{array}{llll}H & -2.422325 & 0.621722 & 0.621722\end{array}$

\section{F (B3LYP-D3(BJ)/def2-TZVPP)}

Imaginary frequencies- 0

Electronic Energy (SCF) $=-490.210505060$ a.u

Grimme-D3(BJ) Dispersion energy $=-0.0533374711 \mathrm{a} . \mathrm{u}$ ZPV corrected Energy = -489.975341 a.u.

Enthalpy $=-489.966593$ a.u.

Free Energy $=-490.006125$ a.u.

C $\quad 0.000000 \quad 1.449660 \quad-0.794967$

H $\quad 0.000000 \quad 2.481467-1.151550$

C $\quad-1.254008 \quad 0.724002 \quad-1.305119$

H $\quad-2.152764 \quad 1.242899 \quad-0.962839$

H $\quad-1.272844 \quad 0.734877 \quad-2.397584$

C $1.254008 \quad 0.724002 \quad-1.305119$

H $\quad 1.272844 \quad 0.734877 \quad-2.397584$

H $\quad 2.152764 \quad 1.242899-0.962839$

C $\quad 0.000000 \quad 1.443706 \quad 0.743626$

H $0.881546 \quad 1.9564391 .133330$

H $\quad-0.881546 \quad 1.9564391 .133330$

C $-1.255442-0.724830-0.794967$

H $\quad-2.149013-1.240734-1.151550$

C $\quad 1.255442-0.724830-0.794967$

H $\quad 2.149013 \quad-1.240734-1.151550$

$\begin{array}{llll}\text { C } & -0.000000 & 0.000000 & 1.229868\end{array}$

C $-1.250286-0.7218530 .743626$

H $\quad-2.135099-0.214779 \quad 1.133330$

H $-1.253553-1.7416601 .133330$

C $1.250286-0.7218530 .743626$

H $\quad 2.135099 \quad-0.214779 \quad 1.133330$

H $1.253553-1.7416601 .133330$

C $-0.000000-1.448004-1.305119$

H $\quad-0.000000 \quad-2.485797 \quad-0.962839$

H $\quad 0.000000 \quad-1.469753 \quad-2.397584$

$\begin{array}{llll}\text { F } & -0.000000 & 0.000000 & 2.641445\end{array}$

\section{Cl (B3LYP-D3(BJ)/def2-TZVPP)}

Imaginary frequencies- 0

Electronic Energy (SCF) = -850.557315335 a.u

Grimme-D3(BJ) Dispersion energy $=-0.0583108332 \mathrm{a} . \mathrm{u}$ ZPV corrected Energy $=-850.323389$ a.u.

Enthalpy $=-850.314220$ a.u.

Free Energy $=-850.355043$ a.u.

C $\quad 0.000000 \quad 1.447722 \quad-1.103858$

H $\quad 0.000000 \quad 2.481199-1.456106$

C $\quad-1.253982 \quad 0.723987-1.614081$

H $\quad-2.152412 \quad 1.242696-1.270583$

H $\quad-1.273742 \quad 0.735395-2.706538$

C $1.253982 \quad 0.723987 \quad-1.614081$

H $\quad 1.273742 \quad 0.735395 \quad-2.706538$

H $\quad 2.152412 \quad 1.242696-1.270583$

C $\quad 0.000000 \quad 1.4460520 .435463$

H $\quad 0.880025 \quad 1.963876 \quad 0.819588$

H $\quad-0.880025 \quad 1.963876 \quad 0.819588$

C $-1.253764-0.723861-1.103858$

H $\quad-2.148782 \quad-1.240600-1.456106$

C $1.253764-0.723861-1.103858$ 
$\begin{array}{llll}\text { H } & 2.148782 & -1.240600 & -1.456106\end{array}$

$\begin{array}{llll}\text { C } & 0.000000 & 0.000000 & 0.926887\end{array}$

$\begin{array}{llll}\text { C } & -1.252318 & -0.723026 & 0.435463\end{array}$

$\begin{array}{llll}\text { H } & -2.140779 & -0.219814 & 0.819588\end{array}$

$\begin{array}{llll}\text { H } & -1.260754 & -1.744062 & 0.819588\end{array}$

$\begin{array}{llll}\text { C } & 1.252318 & -0.723026 & 0.435463\end{array}$

$\begin{array}{llll}\text { H } & 2.140779 & -0.219814 & 0.819588\end{array}$

$\begin{array}{llll}\text { H } & 1.260754 & -1.744062 & 0.819588\end{array}$

$\begin{array}{llll}\text { C } & -0.000000 & -1.447974 & -1.614081\end{array}$

$\begin{array}{llll}\text { H } & -0.000000 & -2.485391 & -1.270583\end{array}$

$\begin{array}{llll}\mathrm{H} & -0.000000 & -1.470791 & -2.706538\end{array}$

$\begin{array}{llll}\text { Cl } & 0.000000 & 0.000000 & 2.759141\end{array}$

\section{Br (B3LYP-D3(BJ)/def2-TZVPP)}

Imaginary frequencies- 0

Electronic Energy (SCF) = -2964.51345625 a.u Grimme-D3(BJ) Dispersion energy $=-0.0597542323 \mathrm{a} . \mathrm{u}$ ZPV corrected Energy = -2964.280071 a.u.

Enthalpy $=-2964.270628$ a.u.

Free Energy $=-2964.312810$ a.u.

$\begin{array}{llll}C & 0.000000 & 1.447374 & -1.609180\end{array}$

$\begin{array}{llll}\text { H } & 0.000000 & 2.481450 & -1.959731\end{array}$

$\begin{array}{llll}\text { C } & -1.254108 & 0.724060 & -2.118839\end{array}$

H $\quad-2.152335 \quad 1.242651 \quad-1.774689$

$\begin{array}{llll}\mathrm{H} & -1.274574 & 0.735876 & -3.211284\end{array}$

$\begin{array}{llll}\text { C } & 1.254108 & 0.724060 & -2.118839\end{array}$

H $\quad 1.274574 \quad 0.735876 \quad-3.211284$

H $\quad 2.152335 \quad 1.242651 \quad-1.774689$

$\begin{array}{llll}\text { C } & 0.000000 & 1.446599 & -0.068037\end{array}$

$\begin{array}{llll}\text { H } & 0.879930 & 1.966241 & 0.313001\end{array}$

$\begin{array}{llll}H & -0.879930 & 1.966241 & 0.313001\end{array}$

$\begin{array}{llll}\text { C } & -1.253463 & -0.723687 & -1.609180\end{array}$

$\begin{array}{llll}\text { H } & -2.148999 & -1.240725 & -1.959731\end{array}$

C $1.253463-0.723687 \quad-1.609180$

H $2.148999 \quad-1.240725 \quad-1.959731$

$\begin{array}{llll}\text { C } & 0.000000 & 0.000000 & 0.416303\end{array}$

$\begin{array}{llll}\text { C } & -1.252791 & -0.723299 & -0.068037\end{array}$

$\begin{array}{llll}\text { H } & -2.142780 & -0.221079 & 0.313001\end{array}$

$\begin{array}{llll}\text { H } & -1.262850 & -1.745162 & 0.313001\end{array}$

$\begin{array}{llll}\text { C } & 1.252791 & -0.723299 & -0.068037\end{array}$

$\begin{array}{llll}\text { H } & 2.142780 & -0.221079 & 0.313001\end{array}$

$\begin{array}{llll}\text { H } & 1.262850 & -1.745162 & 0.313001\end{array}$

$\begin{array}{llll}\text { C } & -0.000000 & -1.448119 & -2.118839\end{array}$

$\begin{array}{llll}\text { H } & -0.000000 & -2.485303 & -1.774689\end{array}$

$\begin{array}{llll}\mathrm{H} & -0.000000 & -1.471752 & -3.211284\end{array}$

$\begin{array}{llll}\mathrm{Br} & 0.000000 & 0.000000 & 2.422580\end{array}$

\section{I (B3LYP-D3(BJ)/def2-TZVPP)}

Imaginary frequencies- 0

Electronic Energy (SCF) = -688.131230612 a.u Grimme-D3(BJ) Dispersion energy $=-0.0618710377 \mathrm{a} . \mathrm{u}$ ZPV corrected Energy = -687.898242 a.u.

Enthalpy $=-687.888634$ a.u.

Free Energy $=-687.931803$ a.u.

$\begin{array}{rrrr}\mathrm{C} & 0.000000 & 1.446916 & -2.037112 \\ \mathrm{H} & 0.000000 & 2.481460 & -2.386679 \\ \mathrm{C} & -1.254142 & 0.724079 & -2.546280 \\ \mathrm{H} & -2.152188 & 1.242566 & -2.201384\end{array}$ $\begin{array}{llll}\text { H } & -1.275431 & 0.736371 & -3.638769\end{array}$

C $1.2541420 .724079-2.546280$

H $\quad 1.2754310 .736371 \quad-3.638769$

$\begin{array}{llll}\text { H } & 2.152188 & 1.242566 & -2.201384\end{array}$

C $0.000000 \quad 1.447265-0.494743$

H $\quad 0.879567 \quad 1.970379 \quad-0.117752$

$\begin{array}{llll}\text { H } & -0.879567 & 1.970379 & -0.117752\end{array}$

$\begin{array}{llll}\text { C } & -1.253066 & -0.723458 & -2.037112\end{array}$

$\begin{array}{llll}\text { H } & -2.149007 & -1.240730 & -2.386679\end{array}$

$\begin{array}{llll}\text { C } & 1.253066 & -0.723458 & -2.037112\end{array}$

H $\quad 2.149007 \quad-1.240730 \quad-2.386679$

$\begin{array}{llll}\text { C } & 0.000000 & 0.000000 & -0.009752\end{array}$

$\begin{array}{llll}\text { C } & -1.253368 & -0.723633 & -0.494743\end{array}$

$\begin{array}{llll}\text { H } & -2.146182 & -0.223462 & -0.117752\end{array}$

$\begin{array}{llll}\text { H } & -1.266614 & -1.746917 & -0.117752\end{array}$

$\begin{array}{llll}\text { C } & 1.253368 & -0.723633 & -0.494743\end{array}$

H $\quad 2.146182-0.223462 \quad-0.117752$

$\begin{array}{llll}\text { H } & 1.266614 & -1.746917 & -0.117752\end{array}$

$\begin{array}{llll}\text { C } & -0.000000 & -1.448159 & -2.546280\end{array}$

$\begin{array}{llll}\text { H } & -0.000000 & -2.485132 & -2.201384\end{array}$

H $\quad-0.000000 \quad-1.472741-3.638769$

I $0.000000 \quad 0.000000 \quad 2.204754$

\section{CCH (B3LYP-D3(BJ)/def2-TZVPP)}

Imaginary frequencies- 0

Electronic Energy (SCF) = -467.097603370 a.u

Grimme-D3(BJ) Dispersion energy $=-0.0601903273 \mathrm{a} . \mathrm{u}$

ZPV corrected Energy $=-466.845415$ a.u.

Enthalpy $=-466.835324$ a.u.

Free Energy $=-466.877778$ a.u.

$\begin{array}{llll}C & 0.000000 & 1.447947 & -1.037045\end{array}$

H $\quad 0.000000 \quad 2.479699-1.395645$

C $-1.253419 \quad 0.723662 \quad-1.547811$

$\begin{array}{llll}\text { H } & -2.152224 & 1.242587 & -1.204866\end{array}$

H $\quad-1.272747 \quad 0.734821 \quad-2.640547$

C $1.2534190 .723662 \quad-1.547811$

$\begin{array}{llll}\text { H } & 1.272747 & 0.734821 & -2.640547\end{array}$

H $\quad 2.152224 \quad 1.242587 \quad-1.204866$

C $\quad 0.000000 \quad 1.452455 \quad 0.496700$

H $\quad 0.878739 \quad 1.975952 \quad 0.878259$

H $\quad-0.878739 \quad 1.9759520 .878259$

C $-1.253959-0.723973-1.037045$

H $\quad-2.147483 \quad-1.239850-1.395645$

C $1.253959-0.723973-1.037045$

H $\quad 2.147483-1.239850-1.395645$

$\begin{array}{llll}\text { C } & 0.000000 & 0.000000 & 1.032937\end{array}$

C $-1.257863 \quad-0.726228 \quad 0.496700$

$\begin{array}{llll}\text { H } & -2.150594 & -0.226966 & 0.878259\end{array}$

H $\quad-1.271856 \quad-1.748986 \quad 0.878259$

C $1.257863 \quad-0.7262280 .496700$

H $\quad 2.150594 \quad-0.226966 \quad 0.878259$

H $\quad \begin{array}{llll}1.271856 & -1.748986 & 0.878259\end{array}$

C $-0.000000-1.447324-1.547811$

H $\quad-0.000000-2.485174-1.204866$

$\begin{array}{llll}\mathrm{H} & -0.000000 & -1.469641 & -2.640547\end{array}$

C $0.000000 \quad 0.000000 \quad 2.490405$

C $\quad 0.000000 \quad 0.000000 \quad 3.691321$

H $\quad 0.000000 \quad 0.000000 \quad 4.752436$

2CN (B3LYP-D3(BJ)/def2-TZVPP) 
Imaginary frequencies- 0

Electronic Energy (SCF) $=-483.201929547$ a.u Grimme-D3(BJ) Dispersion energy $=-0.0589943493 \mathrm{a} . \mathrm{u}$ ZPV corrected Energy = -482.959955 a.u.

Enthalpy $=-482.950171$ a.u.

Free Energy $=-482.992185$ a.u.

C $\quad 0.000000 \quad 1.448314 \quad-1.019249$

H $\quad 0.000000 \quad 2.480049-1.376366$

C $\quad-1.253994 \quad 0.723994-1.528817$

H $\quad-2.152739 \quad 1.242885-1.186300$

H $\quad-1.273170 \quad 0.735065-2.621133$

C $1.253994 \quad 0.723994 \quad-1.528817$

H $\quad 1.273170 \quad 0.735065-2.621133$

H $\quad 2.152739 \quad 1.242885-1.186300$

$\begin{array}{llll}\text { C } & 0.000000 & 1.456113 & 0.514605\end{array}$

H $\quad 0.879074 \quad 1.978516 \quad 0.895849$

H $\quad-0.879074 \quad 1.978516 \quad 0.895849$

C $-1.254277-0.724157-1.019249$

H $\quad-2.147786-1.240025-1.376366$

$\begin{array}{llll}\text { C } & 1.254277 & -0.724157 & -1.019249\end{array}$

H $\quad 2.147786-1.240025-1.376366$

C $\quad 0.000000 \quad 0.000000 \quad 1.038610$

$\begin{array}{llll}\text { C } & -1.261031 & -0.728057 & 0.514605\end{array}$

H $\quad-2.152982 \quad-0.227957 \quad 0.895849$

H $\quad-1.273908 \quad-1.7505590 .895849$

C $1.261031-0.728057 \quad 0.514605$

H $\quad 2.152982 \quad-0.227957 \quad 0.895849$

H $\quad 1.273908 \quad-1.7505590 .895849$

C $-0.000000-1.447988-1.528817$

H $\quad-0.000000-2.485769-1.186300$

H $\quad-0.000000-1.470130-2.621133$

C $\quad 0.000000 \quad 0.000000 \quad 2.499335$

N $\quad 0.000000 \quad 0.000000 \quad 3.650132$

\section{Me (B3LYP-D3(BJ)/def2-TZVPP)}

Imaginary frequencies- 0

Electronic Energy (SCF) $=-430.257815710$ a.u

Grimme-D3(BJ) Dispersion energy $=-0.0599727874$ a.u

ZPV corrected Energy $=-429.987132$ a.u.

Enthalpy $=-429.977718$ a.u.

Free Energy $=-430.018316$ a.u.

C $\quad-0.000000 \quad 1.448171-0.827379$

H $\quad-0.000000 \quad 2.479735-1.187400$

C $-1.252952 \quad 0.723392-1.339051$

H $\quad-2.152066 \quad 1.242496-0.996342$

H $\quad-1.272476 \quad 0.734664-2.432055$

C $1.252952 \quad 0.723392 \quad-1.339051$

H $\quad 1.272476 \quad 0.734664 \quad-2.432055$

H $\quad 2.152066 \quad 1.242496-0.996342$

C $\quad-0.000000 \quad 1.442119 \quad 0.707081$

H $\quad 0.878965 \quad 1.9729411 .084520$

H $\quad-0.878965 \quad 1.972941 \quad 1.084520$

C $-1.254153 \quad-0.724086-0.827379$

H $\quad-2.147513-1.239867-1.187400$

C $1.254153-0.724086-0.827379$

H $\quad 2.147513 \quad-1.239867 \quad-1.187400$

C $\quad 0.000000 \quad 0.000000 \quad 1.247692$

$\begin{array}{llll}\text { C } & -1.248911 & -0.721059 & 0.707081\end{array}$

H $\quad-2.148100 \quad-0.225265 \quad 1.084520$

H $\quad-1.269135 \quad-1.747676 \quad 1.084520$
C $\quad 1.248911 \quad-0.7210590 .707081$

H $\quad 2.148100 \quad-0.225265 \quad 1.084520$

H $\quad \begin{array}{lll}1.269135 & -1.747676 & 1.084520\end{array}$

C $-0.000000-1.446784-1.339051$

H $\quad-0.000000-2.484992-0.996342$

H $\quad-0.000000-1.469328-2.432055$

C $\quad 0.000000 \quad 0.000000 \quad 2.771933$

H $\quad 0.8829450 .5097693 .163603$

H $\quad-0.882945 \quad 0.509769 \quad 3.163603$

H $\quad-0.000000 \quad-1.019537 \quad 3.163603$

\section{Et (B3LYP-D3(BJ)/def2-TZVPP)}

Imaginary frequencies- 0

Electronic Energy (SCF) = -469.589439634 a.u

Grimme-D3(BJ) Dispersion energy $=-0.0663878301 \mathrm{a} . \mathrm{u}$ ZPV corrected Energy = -469.290097 a.u.

Enthalpy $=-469.279287$ a.u.

Free Energy $=-469.324322$ a.u.

C $-0.987522-0.832103-1.254132$

H $\quad-1.228121 \quad-1.413954-2.147124$

C $\quad-1.799517 \quad 0.470077 \quad-1.253197$

H $\quad-1.576425 \quad 1.051415 \quad-2.151883$

H $\quad-2.869308 \quad 0.245590-1.272957$

C $-1.331500-1.6479750 .000001$

$\begin{array}{llll}\mathrm{H} & -2.393940 & -1.905596 & 0.000001\end{array}$

H $\quad-0.773117-2.5880090 .000002$

C $0.512135-0.504065-1.250153$

H $\quad 1.086621-1.432323 \quad-1.278563$

H $\quad 0.775390 \quad 0.062302-2.148705$

$\begin{array}{llll}\text { C } & -1.454329 & 1.286047 & -0.000001\end{array}$

H $\quad-2.023990 \quad 2.218459-0.000002$

C $\quad-0.987522 \quad-0.832102 \quad 1.254133$

H $\quad-1.228122 \quad-1.413951 \quad 2.147126$

C $0.889508 \quad 0.313840 \quad 0.000000$

C $\quad 0.045595 \quad 1.603893 \quad-0.000001$

H $\quad 0.301046 \quad 2.203548 \quad-0.878683$

$\begin{array}{llll}H & 0.301046 & 2.203550 & 0.878680\end{array}$

C $0.512135-0.504063 \quad 1.250154$

H $\quad 1.086621 \quad-1.432321 \quad 1.278565$

H $\quad 0.7753890 .062304 \quad 2.148706$

C $\quad-1.799518 \quad 0.470079 \quad 1.253196$

H $-1.576425 \quad 1.051418 \quad 2.151881$

$\begin{array}{llll}H & -2.869309 & 0.245592 & 1.272955\end{array}$

C 2.3764430 .6983710 .000000

H $\quad 2.561065 \quad 1.330773-0.874145$

H $\quad 2.561065 \quad 1.330774 \quad 0.874145$

C $3.381152-0.4523650 .000000$

H $\quad 4.401031 \quad-0.065724-0.000000$

H $\quad 3.273560 \quad-1.0858290 .880721$

H $\quad 3.273559-1.085829 \quad-0.880721$

\section{2iPr (B3LYP-D3(BJ)/def2-TZVPP)}

Imaginary frequencies- 0

Electronic Energy (SCF) = -508.920929511 a.u

Grimme-D3(BJ) Dispersion energy $=-0.0740421842 \mathrm{a} . \mathrm{u}$

ZPV corrected Energy $=-508.593472$ a.u.

Enthalpy $=-508.581216$ a.u.

Free Energy $=-508.630007$ a.u.

C $\quad-1.692727 \quad-0.947167 \quad 0.000000$ 
$\begin{array}{llll}\text { H } & -2.784484 & -0.992526 & 0.000000\end{array}$

$\begin{array}{llll}\text { C } & -1.148736 & -1.648977 & 1.251324\end{array}$

H $\quad-1.543487 \quad-1.170077 \quad 2.151471$

H $\quad-1.478631 \quad-2.691092 \quad 1.270670$

C $-1.148736-1.648977-1.251324$

H $\quad-1.478631-2.691092-1.270670$

H $\quad-1.543487-1.170077-2.151471$

C $-1.2503290 .524477 \quad-0.000000$

H $\quad-1.662294 \quad 1.026592 \quad-0.877949$

H $\quad-1.662294 \quad 1.026592 \quad 0.877949$

$\begin{array}{llll}\text { C } & 0.384425 & -1.579832 & 1.248957\end{array}$

H $\quad 0.774971 \quad-2.067446 \quad 2.145497$

$\begin{array}{llll}\text { C } & 0.384425 & -1.579832 & -1.248957\end{array}$

H $\quad 0.774971 \quad-2.067446 \quad-2.145497$

C $\quad 0.287700 \quad 0.634219 \quad-0.000000$

$\begin{array}{llll}\text { C } & 0.820054 & -0.108962 & 1.244836\end{array}$

$\begin{array}{llll}\text { H } & 0.452020 & 0.369274 & 2.153277\end{array}$

H $\quad 1.912044-0.040080 \quad 1.267600$

C $0.820054-0.108962-1.244836$

H $\quad 0.452020 \quad 0.369274-2.153277$

H $\quad 1.912044-0.040080-1.267600$

C $0.927758-2.282957 \quad 0.000000$

H $\quad 2.020792 \quad-2.255961 \quad 0.000000$

H $\quad 0.631821-3.3354460 .000000$

C $0.789127 \quad 2.105628-0.000000$

H $\quad 1.883585 \quad 2.039457 \quad-0.000000$

C $0.384425 \quad 2.901496-1.246103$

H $\quad 0.813970 \quad 3.903333 \quad-1.202893$

H $\quad 0.728707 \quad 2.439676-2.169422$

H $\quad-0.698753 \quad 3.017109-1.310625$

C $0.384425 \quad 2.901496 \quad 1.246103$

$\begin{array}{llll}\text { H } & 0.728707 & 2.439676 & 2.169422\end{array}$

H $\quad 0.813970 \quad 3.903333 \quad 1.202893$

H $\quad-0.698753 \quad 3.017109 \quad 1.310625$

\section{2tBu (B3LYP-D3(BJ)/def2-TZVPP)}

Imaginary frequencies- 0

Electronic Energy (SCF) = -548.251976247 a.u

Grimme-D3(BJ) Dispersion energy $=-0.0829499629$ a.u

ZPV corrected Energy $=-547.896345$ a.u.

Enthalpy $=-547.882975$ a.u.

Free Energy $=-547.931948$ a.u.
C $0.009317 \quad 1.442586-1.610970$
H $\quad 0.015660 \quad 2.476989-1.963354$
C $\quad-1.247422 \quad 0.730671-2.125362$
H $\quad-2.142625 \quad 1.255849-1.781400$
H $\quad-1.267795 \quad 0.741798-3.218408$
C $1.256491 \quad 0.714964-2.125362$
H $\quad 1.276313 \quad 0.727043 \quad-3.218408$
H $\quad 2.158910 \quad 1.227643 \quad-1.781400$
C $\quad 0.000000 \quad 1.439581 \quad-0.075756$
H $\quad 0.869099 \quad 1.988478 \quad 0.292074$
$\begin{array}{llll}\text { H } & -0.884568 & 1.975800 & 0.269262\end{array}$
C $-1.253975-0.713224-1.610970$
H $\quad-2.152965-1.224933-1.963354$
C $1.244658-0.729362-1.610970$
H $\quad 2.137305 \quad-1.252056-1.963354$
$\begin{array}{llll}\text { C } & 0.000000 & 0.000000 & 0.489833\end{array}$
$\begin{array}{llll}\text { C } & -1.246714 & -0.719790 & -0.075756\end{array}$
$\begin{array}{llll}\text { H } & -2.156622 & -0.241577 & 0.292074\end{array}$

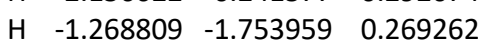

$\begin{array}{llll}\text { C } & 1.246714 & -0.719790 & -0.075756\end{array}$

H $\quad 2.153377 \quad-0.221842 \quad 0.269262$

H $\quad \begin{array}{llll}1.287523 & -1.746901 & 0.292074\end{array}$

C $-0.009069-1.445635-2.125362$

H $\quad-0.016285-2.483492-1.781400$

H $\quad-0.008519-1.468841 \quad-3.218408$

C $\quad 0.000000 \quad 0.000000 \quad 2.068802$

C $\quad 1.336654 \quad 0.514828 \quad 2.631143$

H $1.2591290 .624756 \quad 3.713995$

$\begin{array}{llll}\text { H } & 2.155802 & -0.174874 & 2.432851\end{array}$

H $\quad 1.607033 \quad 1.488446 \quad 2.223378$

C $\quad-1.114181 \quad 0.900163 \quad 2.631143$

$\begin{array}{llll}\text { H } & -2.092548 & 0.647509 & 2.223378\end{array}$

H $\quad-1.1706190 .778060 \quad 3.713995$

H $\quad-0.926455 \quad 1.954416 \quad 2.432851$

C $-0.222473-1.414990 \quad 2.631143$

H $\quad-0.088510 \quad-1.402816 \quad 3.713995$

H $\quad-1.229346-1.779542 \quad 2.432851$

H $\quad 0.485515 \quad-2.135954 \quad 2.223378$

\section{Ph (B3LYP-D3(BJ)/def2-TZVPP)}

Imaginary frequencies- 0

Electronic Energy (SCF) = -622.079754840 a.u

Grimme-D3(BJ) Dispersion energy $=-0.0827207982 \mathrm{a} . \mathrm{u}$

ZPV corrected Energy $=-621.755480$ a.u.

Enthalpy $=-621.742935$ a.u.

Free Energy $=-621.792719$ a.u.

$\begin{array}{llll}C & -2.210317 & 0.074816 & 1.254090\end{array}$

H $\quad-2.720756-0.292701 \quad 2.147436$

C $-2.931746-0.438194 \quad 0.000000$

H $\quad-2.951487-1.5313690 .000000$

H $\quad-3.970670 \quad-0.098404 \quad 0.000000$

C $\quad-2.210317 \quad 1.608435 \quad 1.252512$

H $\quad-3.236096 \quad 1.985795 \quad 1.268718$

H $\quad-1.714863 \quad 1.984140 \quad 2.151650$

C $-0.764195 \quad-0.435921 \quad 1.255958$

$\begin{array}{llll}\text { H } & -0.239282 & -0.088994 & 2.149538\end{array}$

H $\quad-0.758859-1.526572 \quad 1.290241$

C $-2.210317 \quad 0.074816-1.254090$

H $\quad-2.720756-0.292701-2.147436$

C $-1.482829 \quad 2.1132090 .000000$

H $\quad-1.467196 \quad 3.2055810 .000000$

C $-0.002732 \quad 0.0541710 .000000$

C $-0.764195-0.435921-1.255958$

H $\quad-0.758859-1.526572 \quad-1.290241$

H $\quad-0.239282-0.088994-2.149538$

C $\quad-0.038541 \quad 1.5946890 .000000$

H $\quad 0.485607 \quad 1.976870 \quad 0.879295$

H $\quad 0.485607 \quad 1.976870-0.879295$

C $\quad-2.210317 \quad 1.608435 \quad-1.252512$

H $\quad-1.714863 \quad 1.984140 \quad-2.151650$

H $\quad-3.236096 \quad 1.985795-1.268718$

C $1.414392-0.5049590 .000000$

C $1.614764 \quad-1.8899090 .000000$

C $2.547965 \quad 0.307577 \quad 0.000000$

C $2.888177-2.439103 \quad 0.000000$

H $\quad 0.762077 \quad-2.5548840 .000000$

C $3.828938-0.237496 \quad 0.000000$

H $\quad 2.445170 \quad 1.381893 \quad 0.000000$

C $4.006825 \quad-1.6127290 .000000$

H $3.007910-3.5145610 .000000$ 
$\begin{array}{llll}H & 4.687857 & 0.420714 & 0.000000\end{array}$

H $\quad \begin{array}{llll}5.001515 & -2.037534 & 0.000000\end{array}$

\section{CF 3 (B3LYP-D3(BJ)/def2-TZVPP)}

Imaginary frequencies- 0

Electronic Energy (SCF) = -728.126709062 a.u Grimme-D3(BJ) Dispersion energy $=-0.0617640617 \mathrm{a} . \mathrm{u}$ ZPV corrected Energy = -727.878458 a.u.

Enthalpy $=-727.867021$ a.u.

Free Energy $=-727.913012$ a.u.

C $\quad 0.000000 \quad 1.447085-1.553036$

H $\quad 0.000000 \quad 2.479353-1.908788$

C $\quad-1.253462 \quad 0.723686-2.063877$

H $\quad-2.152240 \quad 1.242597 \quad-1.721186$

H $\quad-1.272475 \quad 0.734664 \quad-3.156366$

C $\quad 1.253462 \quad 0.723686-2.063877$

H $\quad 1.2724750 .734664-3.156366$

H $\quad 2.152240 \quad 1.242597 \quad-1.721186$

$\begin{array}{llll}\text { C } & 0.000000 & 1.451490 & -0.018257\end{array}$

H $\quad 0.879393 \quad 1.977975 \quad 0.355706$

H $\quad-0.879393 \quad 1.977975 \quad 0.355706$

C $\quad-1.253212-0.723542-1.553036$

H $\quad-2.147183-1.239677-1.908788$

C $1.253212-0.723542-1.553036$

$\begin{array}{llll}\text { H } & 2.147183 & -1.239677 & -1.908788\end{array}$

C $0.000000 \quad 0.000000 \quad 0.503109$

$\begin{array}{llll}\text { C } & -1.257027 & -0.725745 & -0.018257\end{array}$

H $\quad-2.152673 \quad-0.227411 \quad 0.355706$

H $\quad-1.273280-1.750565 \quad 0.355706$

$\begin{array}{llll}\text { C } & 1.257027 & -0.725745 & -0.018257\end{array}$

H $\quad 2.152673 \quad-0.2274110 .355706$

H $\quad 1.273280-1.7505650 .355706$

C $\quad-0.000000 \quad-1.447373 \quad-2.063877$

H $\quad-0.000000-2.485193-1.721186$

H $\quad-0.000000-1.469328-3.156366$

C $\quad 0.000000 \quad 0.000000 \quad 2.023825$

$\begin{array}{llll}\text { F } & -1.083712 & 0.625681 & 2.536898\end{array}$

$\begin{array}{llll}F & -0.000000 & -1.251363 & 2.536898\end{array}$

$\begin{array}{llll}F & 1.083712 & 0.625681 & 2.536898\end{array}$

\section{$2 \mathrm{CCl}_{3}$ (B3LYP-D3(BJ)/def2-TZVPP)}

Imaginary frequencies- 0

Electronic Energy (SCF) = -1809.12872128 a.u Grimme-D3(BJ) Dispersion energy $=-0.0778570181 \mathrm{a} . \mathrm{u}$ ZPV corrected Energy $=-1808.885726$ a.u.

Enthalpy $=-1808.872990$ a.u.

Free Energy $=-1808.922194$ a.u.

$\begin{array}{llll}\text { C } & 0.000000 & 1.443227 & -2.093414\end{array}$

H $\quad 0.000000 \quad 2.477996-2.442215$

C $1.2529810 .723409-2.605973$

H $\quad 2.151384 \quad 1.242102-2.262116$

H $\quad \begin{array}{llll}1.272238 & 0.734527 & -3.698477\end{array}$

$\begin{array}{llll}\text { C } & -1.252981 & 0.723409 & -2.605973\end{array}$

H $\quad-1.272238 \quad 0.734527 \quad-3.698477$

H $\quad-2.151384 \quad 1.242102-2.262116$

C $0.000000 \quad 1.448683 \quad-0.558432$

H $\quad-0.877979 \quad 1.978391-0.190446$

H $\quad 0.877979 \quad 1.978391-0.190446$

C $1.249871-0.721613 \quad-2.093414$
H $\quad 2.146008 \quad-1.238998-2.442215$

C $-1.249871-0.721613-2.093414$

H $\quad-2.146008-1.238998-2.442215$

$\begin{array}{llll}\text { C } & 0.000000 & 0.000000 & -0.021004\end{array}$

C $1.254597-0.724342-0.558432$

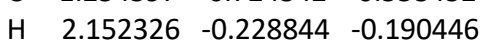

H $\quad \begin{array}{llll}1.274348 & -1.749547 & -0.190446\end{array}$

C $-1.254597-0.724342-0.558432$

H $-2.152326 \quad-0.228844-0.190446$

H $\quad-1.274348-1.749547-0.190446$

C $-0.000000-1.446818-2.605973$

H $\quad-0.000000 \quad-2.484204-2.262116$

H $\quad-0.000000-1.469054-3.698477$

C $\quad 0.000000 \quad 0.000000 \quad 1.528161$

$\begin{array}{llll}\text { Cl } & 1.448920 & 0.836534 & 2.195076\end{array}$

$\begin{array}{llll}\mathrm{Cl} & -1.448920 & 0.836534 & 2.195076\end{array}$

Cl $\quad-0.000000 \quad-1.673069 \quad 2.195076$

\section{CBr 3 (B3LYP-D3(BJ)/def2-TZVPP)}

Imaginary frequencies- 0

Electronic Energy (SCF) $=-8150.99354340$ a.u

Grimme-D3(BJ) Dispersion energy $=-0.0832431949$ a.u ZPV corrected Energy $=-8150.752516$ a.u.

Enthalpy $=-8150.738795$ a.u.

Free Energy $=-8150.791876$ a.u.

C $\quad-0.000000 \quad 1.442297 \quad-2.776593$

H $\quad-0.000000 \quad 2.477749 \quad-3.123407$

C $\quad 1.252983 \quad 0.723410-3.289255$

H $\quad 2.151220 \quad 1.242008 \quad-2.944989$

H $\quad 1.2723620 .734599-4.381736$

$\begin{array}{llll}\text { C } & -1.252983 & 0.723410 & -3.289255\end{array}$

H $\quad-1.2723620 .734599 \quad-4.381736$

H $\quad-2.151220 \quad 1.242008 \quad-2.944989$

C $\quad-0.000000 \quad 1.448444 \quad-1.241638$

H $\quad-0.877609 \quad 1.979084-0.874927$

$\begin{array}{llll}H & 0.877609 & 1.979084 & -0.874927\end{array}$

$\begin{array}{llll}\text { C } & 1.249066 & -0.721148 & -2.776593\end{array}$

H $\quad 2.145793 \quad-1.238874-3.123407$

C $\quad-1.249066-0.721148-2.776593$

$\begin{array}{llll}\text { H } & -2.145793 & -1.238874 & -3.123407\end{array}$

C $-0.000000-0.000000-0.698692$

C $1.254389-0.724222-1.241638$

H $\quad 2.152741-0.229510 \quad-0.874927$

H $\quad \begin{array}{llll}\text { H.275132 } & -1.749574 & -0.874927\end{array}$

C $-1.254389-0.724222-1.241638$

H $\quad-2.152741-0.229510-0.874927$

H $\quad-1.275132-1.749574-0.874927$

C $-0.000000-1.446820-3.289255$

H $\quad-0.000000-2.484015 \quad-2.944989$

H $\quad-0.000000-1.469197-4.381736$

C $\quad 0.000000 \quad 0.000000 \quad 0.847843$

$\begin{array}{llll}\mathrm{Br} & 0.000000 & -1.833142 & 1.592760\end{array}$

$\begin{array}{llll}\mathrm{Br} & -1.587548 & 0.916571 & 1.592760\end{array}$

$\begin{array}{llll}\mathrm{Br} & 1.587548 & 0.916571 & 1.592760\end{array}$

\section{Cl 3 (B3LYP-D3(BJ)/def2-TZVPP)}

Imaginary frequencies- 0

Electronic Energy (SCF) = -1321.85145997 a.u Grimme-D3(BJ) Dispersion energy $=-0.0917564625$ a.u ZPV corrected Energy = -1321.611689 a.u. 
Enthalpy $=-1321.597392$ a.u.

Free Energy = -1321.653301 a.u.

$\begin{array}{llll}C & 1.248406 & 0.720767 & 3.203168\end{array}$

H $\quad 2.145724 \quad 1.238835 \quad 3.548032$

C $\quad-0.000000 \quad 1.446915 \quad 3.715760$

H $\quad-0.000000 \quad 2.483910 \quad 3.371044$

H $\quad-0.000000 \quad 1.4695394 .808240$

C $1.253065-0.723457 \quad 3.715760$

H $\quad 1.272658-0.7347694 .808240$

H $\quad 2.151129 \quad-1.241955 \quad 3.371044$

C $1.252925 \quad 0.723376 \quad 1.668113$

H $\quad 2.152749 \quad 0.229912 \quad 1.302881$

H $\quad 1.275484 \quad 1.749379 \quad 1.302881$

C $\quad-1.248406 \quad 0.720767 \quad 3.203168$

H $\quad-2.145724 \quad 1.238835 \quad 3.548032$

C $-0.000000 \quad-1.441535 \quad 3.203168$

$\begin{array}{llll}\text { H } & -0.000000 & -2.477669 & 3.548032\end{array}$

C $-0.000000 \quad-0.000000 \quad 1.118674$

C $\quad-1.252925 \quad 0.723376 \quad 1.668113$

H $\quad-1.275484 \quad 1.749379 \quad 1.302881$

$\begin{array}{llll}\text { H } & -2.152749 & 0.229912 & 1.302881\end{array}$

C $-0.000000 \quad-1.446753 \quad 1.668113$

H $\quad 0.877265 \quad-1.979291 \quad 1.302881$

H $\quad-0.877265 \quad-1.979291 \quad 1.302881$

C $-1.253065-0.723457 \quad 3.715760$

H $\quad-2.151129-1.241955 \quad 3.371044$

H $\quad-1.272658 \quad-0.734769 \quad 4.808240$

C $0.000000 \quad 0.000000-0.429289$

I $\quad-0.000000 \quad 2.036034 \quad-1.268568$

I $1.763258-1.018017 \quad-1.268568$

I $\begin{array}{llll}-1.763258 & -1.018017 & -1.268568\end{array}$

\section{SiMe 3 (B3LYP-D3(BJ)/def2-TZVPP)}

Imaginary frequencies- 0

Electronic Energy (SCF) = -799.698139631 a.u Grimme-D3(BJ) Dispersion energy $=-0.0845871472$ a.u ZPV corrected Energy = -799.354419 a.u.

Enthalpy $=-799.338473$ a.u.

Free Energy $=-799.394020$ a.u.

$\begin{array}{llll}C & -0.000164 & 1.447565 & -1.879661\end{array}$

H $\quad-0.000286 \quad 2.479437 \quad-2.239955$

$\begin{array}{llll}\text { C } & -1.253056 & 0.723269 & -2.390145\end{array}$

H $\quad-2.151833 \quad 1.242015-2.045386$

H $\quad-1.274736 \quad 0.735808 \quad-3.483285$

C $1.252898 \quad 0.723544 \quad-2.390145$

H $1.274596 \quad 0.736050-3.483285$

H $\quad 2.151533 \quad 1.242534-2.045386$

C $\quad-0.000000 \quad 1.445717-0.343464$

H $\quad 0.878163 \quad 1.985693 \quad 0.020586$

H $\quad-0.877997 \quad 1.985832 \quad 0.020827$

C $-1.253546-0.723925-1.879661$

H $\quad-2.147112-1.239966-2.239955$

C $1.253710-0.723640-1.879661$

$\begin{array}{llll}\text { H } & 2.147398 & -1.239471 & -2.239955\end{array}$

$\begin{array}{llll}\text { C } & 0.000000 & 0.000000 & 0.204033\end{array}$

$\begin{array}{llll}\text { C } & -1.252027 & -0.722858 & -0.343464\end{array}$

H $\quad-2.158743 \quad-0.2323350 .020586$

$\begin{array}{llll}\text { H } & -1.280782 & -1.753284 & 0.020827\end{array}$

C $1.252027 \quad-0.722858-0.343464$

$\begin{array}{llll}\text { H } & 2.158780 & -0.232548 & 0.020827\end{array}$
H $\quad 1.280579-1.7533590 .020586$

C $0.000159-1.446813-2.390145$

H $\quad 0.000300 \quad-2.484549-2.045386$

H $\quad 0.000140 \quad-1.471858-3.483285$

C $\quad 1.526997 \quad 0.889504 \quad 2.750345$

H $\quad \begin{array}{llll}\text { H } & 1.533737 & 0.890333 & 3.842182\end{array}$

H $2.446552 \quad 0.406390 \quad 2.416477$

H $\quad 1.557712 \quad 1.9291632 .420802$

$\begin{array}{llll}\text { C } & -1.533832 & 0.877666 & 2.750345\end{array}$

$\begin{array}{llll}\text { H } & -2.449560 & 0.384437 & 2.420802\end{array}$

H $\quad-1.5379190 .8830893 .842182$

$\begin{array}{llll}H & -1.575220 & 1.915581 & 2.416477\end{array}$

C $0.006835-1.767170 \quad 2.750345$

H $\quad 0.004183 \quad-1.773421 \quad 3.842182$

$\begin{array}{llll}H & -0.871332 & -2.321971 & 2.416477\end{array}$

H $\quad 0.891848 \quad-2.3135992 .420802$

Si $\quad 0.000000 \quad 0.000000 \quad 2.103694$

\section{H (B3LYP-D3(BJ)/def2-TZVPP)}

Imaginary frequencies- 0

Electronic Energy (SCF) = -508.928836323 a.u

Grimme-D3(BJ) Dispersion energy $=-0.0696571179$ a.u ZPV corrected Energy $=-508.600220$ a.u.

Enthalpy $=-508.588367$ a.u.

Free Energy $=-508.634745$ a.u.

$\begin{array}{llll}C & 1.259685 & 2.189960 & 0.206308\end{array}$

H $\quad 2.152869 \quad 2.701409-0.161574$

H $\quad 1.318289 \quad 2.210852 \quad 1.300315$

C $\quad 0.000000 \quad 2.926193 \quad-0.245622$

H $\quad 0.000000 \quad 3.002821 \quad-1.337759$

H $\quad 0.000000 \quad 3.9488820 .137650$

$\begin{array}{llll}\text { C } & 1.270311 & 0.733414 & -0.258507\end{array}$

H $\quad 1.268164 \quad 0.732175-1.357006$

$\begin{array}{llll}\text { C } & -1.259685 & 2.189960 & 0.206308\end{array}$

H $\quad-1.3182892 .210852 \quad 1.300315$

H $\quad-2.152869 \quad 2.701409 \quad-0.161574$

C $\quad 0.000000 \quad 0.000000 \quad 0.197956$

H $\quad 0.000000 \quad 0.000000 \quad 1.298399$

C $\quad-1.270311 \quad 0.733414 \quad-0.258507$

H $\quad-1.268164 \quad 0.732175-1.357006$

$\begin{array}{llll}\text { C } & 2.526404 & -0.004061 & 0.206308\end{array}$

H $3.415923 \quad 0.513735-0.161574$

H $2.573798 \quad 0.036245 \quad 1.300315$

$\begin{array}{llll}\text { C } & -2.526404 & -0.004061 & 0.206308\end{array}$

H $\quad-3.415923 \quad 0.513735 \quad-0.161574$

$\begin{array}{llll}H & -2.573798 & 0.036245 & 1.300315\end{array}$

$\begin{array}{llll}\text { C } & -0.000000 & -1.466829 & -0.258507\end{array}$

H $\quad-0.000000-1.464350-1.357006$

$\begin{array}{llll}\text { C } & 1.266719 & -2.185899 & 0.206308\end{array}$

H $\quad 1.255510 \quad-2.247097 \quad 1.300315$

H $\quad 1.263054-3.215144 \quad-0.161574$

$\begin{array}{llll}\text { C } & -1.266719 & -2.185899 & 0.206308\end{array}$

H $\quad-1.255510-2.247097 \quad 1.300315$

H $\quad-1.263054 \quad-3.215144 \quad-0.161574$

$\begin{array}{llll}\text { C } & -2.534157 & -1.463097 & -0.245622\end{array}$

H $\quad-2.600519-1.501410-1.337759$

H $\quad-3.419832 \quad-1.9744410 .137650$

$\begin{array}{llll}\text { C } & 2.534157 & -1.463097 & -0.245622\end{array}$

H $2.600519-1.501410-1.337759$

H $\quad 3.419832 \quad-1.9744410 .137650$ 


\section{F (B3LYP-D3(BJ)/def2-TZVPP)}

Imaginary frequencies- 0

Electronic Energy (SCF) = -608.216492993 a.u

Grimme-D3(BJ) Dispersion energy $=-0.0707226839$ a.u

ZPV corrected Energy = -607.895664 a.u.

Enthalpy $=-607.883010$ a.u.

Free Energy = -607.930917 a.u.

$\begin{array}{llll}C & 1.258580 & 2.193619 & 0.084762\end{array}$

H $\quad 2.152601 \quad 2.695041-0.292813$

H $\quad 1.318738 \quad 2.221163 \quad 1.175583$

C $0.000000 \quad 2.925657-0.376149$

H $\quad 0.000000 \quad 2.993819-1.469266$

H $\quad 0.000000 \quad 3.950839-0.000516$

C $\quad 1.271125 \quad 0.733884 \quad-0.372397$

H $\quad 1.250235 \quad 0.721823 \quad-1.468974$

C $\quad-1.258580 \quad 2.1936190 .084762$

H $\quad-1.318738 \quad 2.221163 \quad 1.175583$

H $\quad-2.152601 \quad 2.695041 \quad-0.292813$

$\begin{array}{llll}\text { C } & 0.000000 & 0.000000 & 0.072252\end{array}$

$\begin{array}{llll}\text { C } & -1.271125 & 0.733884 & -0.372397\end{array}$

H $\quad-1.250235 \quad 0.721823 \quad-1.468974$

$\begin{array}{llll}\text { C } \quad 2.529020 & -0.006847 & 0.084762\end{array}$

$\begin{array}{llll}\text { H } & 3.410274 & 0.516687 & -0.292813\end{array}$

H $\quad 2.582952 \quad 0.031479 \quad 1.175583$

C $-2.529020-0.006847 \quad 0.084762$

$\begin{array}{llll}\text { H } & -3.410274 & 0.516687 & -0.292813\end{array}$

H $\quad-2.582952 \quad 0.031479 \quad 1.175583$

$\begin{array}{llll}\text { C } & -0.000000 & -1.467768 & -0.372397\end{array}$

$\begin{array}{llll}\text { H } & -0.000000 & -1.443647 & -1.468974\end{array}$

C $1.270440 \quad-2.1867720 .084762$

H $\quad 1.264215 \quad-2.252642 \quad 1.175583$

H $\quad \begin{array}{llll}1.257673 & -3.211728 & -0.292813\end{array}$

C $\quad-1.270440 \quad-2.186772 \quad 0.084762$

H $-1.264215-2.252642 \quad 1.175583$

H $\quad-1.257673-3.211728-0.292813$

C $-2.533693 \quad-1.462828-0.376149$

H $\quad-2.592723 \quad-1.496910-1.469266$

H $\quad-3.421527-1.975420-0.000516$

C $2.533693-1.462828-0.376149$

H $2.592723 \quad-1.496910-1.469266$

H $3.421527-1.975420-0.000516$

F $\quad 0.000000 \quad 0.000000 \quad 1.500950$

\section{Cl (B3LYP-D3(BJ)/def2-TZVPP)}

Imaginary frequencies- 0

Electronic Energy (SCF) = -968.562807022 a.u Grimme-D3(BJ) Dispersion energy $=-0.0793323030 \mathrm{a} . \mathrm{u}$ ZPV corrected Energy = -968.243156 a.u.

Enthalpy $=-968.230243$ a.u.

Free Energy $=-968.278753$ a.u.

$\begin{array}{llll}C & 1.266896 & 2.205460 & -0.104214\end{array}$

H $\quad 2.1504792 .687879-0.528082$

H $\quad 1.357321 \quad 2.280143 \quad 0.981146$

C $\quad 0.000000 \quad 2.922634-0.572477$

H $\quad 0.000000 \quad 2.969749-1.666576$

H $\quad 0.000000 \quad 3.955199-0.218107$

C $\quad 1.266120 \quad 0.730994 \quad-0.505929$

H $\quad 1.198450 \quad 0.691925-1.601597$

C $-1.266896 \quad 2.205460 \quad-0.104214$ $\begin{array}{llll}\text { H } & -1.357321 & 2.280143 & 0.981146\end{array}$

H $\quad-2.150479 \quad 2.687879 \quad-0.528082$

$\begin{array}{llll}\text { C } & 0.000000 & 0.000000 & -0.032125\end{array}$

C $\quad-1.266120 \quad 0.730994 \quad-0.505929$

H $\quad-1.198450 \quad 0.691925-1.601597$

C $2.543433 \quad-0.005566-0.104214$

H $3.4030110 .518430 \quad-0.528082$

H $\quad 2.6533220 .0354030 .981146$

C $\quad-2.543433-0.005566 \quad-0.104214$

$\begin{array}{llll}\text { H } & -3.403011 & 0.518430 & -0.528082\end{array}$

H $\quad-2.653322 \quad 0.035403 \quad 0.981146$

C $\quad-0.000000-1.461989-0.505929$

H $\quad-0.000000-1.383850-1.601597$

C $\quad 1.276537-2.199894-0.104214$

H $\quad 1.296001 \quad-2.315546 \quad 0.981146$

H $\quad 1.252532-3.206309-0.528082$

C $-1.276537-2.199894 \quad-0.104214$

H $\quad-1.296001 \quad-2.315546 \quad 0.981146$

H $\quad-1.252532-3.206309-0.528082$

C $-2.531076-1.461317-0.572477$

H $\quad-2.571878-1.484874-1.666576$

H $\quad-3.425303-1.977600-0.218107$

C $2.531076-1.461317-0.572477$

H $\quad 2.571878-1.484874 \quad-1.666576$

H $3.425303 \quad-1.977600-0.218107$

Cl $\quad 0.000000 \quad 0.000000 \quad 1.829190$

\section{Br (B3LYP-D3(BJ)/def2-TZVPP)}

Imaginary frequencies- 0

Electronic Energy (SCF) = -3082.51864130 a.u

Grimme-D3(BJ) Dispersion energy $=-0.0818966601 \mathrm{a} . \mathrm{u}$ ZPV corrected Energy = -3082.199629 a.u.

Enthalpy $=-3082.186420$ a.u.

Free Energy $=-3082.236148$ a.u.

$\begin{array}{llll}\text { C } & 1.268725 & 2.208423 & -0.401678\end{array}$

H $\quad 2.149742 \quad 2.685957-0.836231$

H $\quad 1.366415 \quad 2.294169 \quad 0.681944$

C $\quad 0.000000 \quad 2.922298-0.871223$

H $\quad 0.000000 \quad 2.965539-1.965429$

H $\quad 0.000000 \quad 3.956167-0.520896$

C $1.265436 \quad 0.730600 \quad-0.790489$

H $\quad 1.191554 \quad 0.687944-1.886583$

$\begin{array}{lllll}\text { C } & -1.268725 & 2.208423 & -0.401678\end{array}$

$\begin{array}{llll}\text { H } & -1.366415 & 2.294169 & 0.681944\end{array}$

H $\quad-2.149742 \quad 2.685957 \quad-0.836231$

$\begin{array}{llll}\text { C } & 0.000000 & 0.000000 & -0.322138\end{array}$

C $-1.265436 \quad 0.730600 \quad-0.790489$

H $\quad-1.191554 \quad 0.687944 \quad-1.886583$

$\begin{array}{llll}\text { C } & 2.546913 & -0.005464 & -0.401678\end{array}$

H $\quad 3.400978 \quad 0.518753 \quad-0.836231$

$\begin{array}{llll}\text { H } & 2.670016 & 0.036265 & 0.681944\end{array}$

$\begin{array}{llll}\text { C } & -2.546913 & -0.005464 & -0.401678\end{array}$

$\begin{array}{llll}\text { H } & -3.400978 & 0.518753 & -0.836231\end{array}$

H $\quad-2.670016 \quad 0.036265 \quad 0.681944$

$\begin{array}{llll}\text { C } & -0.000000 & -1.461199 & -0.790489\end{array}$

H $\quad-0.000000-1.375888-1.886583$

C 1.278188 -2.202959 -0.401678

H $\quad 1.303601 \quad-2.3304350 .681944$

H $\quad 1.251236-3.204710-0.836231$

C $\quad-1.278188-2.202959-0.401678$

H $\quad-1.303601-2.330435 \quad 0.681944$ 
H $\quad-1.251236-3.204710-0.836231$

C $-2.530785-1.461149-0.871223$

H $\quad-2.568232-1.482770-1.965429$

H $\quad-3.426141 \quad-1.978084-0.520896$

C $2.530785-1.461149-0.871223$

H $\quad 2.568232-1.482770 \quad-1.965429$

H $\quad 3.426141 \quad-1.978084 \quad-0.520896$

$\mathrm{Br} \quad 0.000000 \quad 0.000000 \quad 1.724243$

\section{I (B3LYP-D3(BJ)/def2-TZVPP)}

Imaginary frequencies- 0

Electronic Energy (SCF) = -806.136228736 a.u Grimme-D3(BJ) Dispersion energy $=-0.0856511381$ a.u ZPV corrected Energy $=-805.817749$ a.u.

Enthalpy $=-805.804368$ a.u.

Free Energy $=-805.854996$ a.u.

C $\quad-1.279565 \quad 2.206069-0.692382$

H $\quad-1.249398 \quad 3.202873 \quad-1.138023$

$\begin{array}{llll}H & -1.312491 & 2.347310 & 0.389420\end{array}$

C $\quad-2.5302891 .460863 \quad-1.163039$

H $\quad-2.564052 \quad 1.480356-2.257274$

H $\quad-3.426872 \quad 1.978506 \quad-0.817073$

C $\quad-0.000000 \quad 1.459328-1.065965$

H $\quad-0.000000 \quad 1.364461-2.162164$

$\begin{array}{llll}\text { C } & -2.550294 & 0.005101 & -0.692382\end{array}$

H $\quad-2.689076 \quad-0.037005 \quad 0.389420$

H $\quad-3.398468 \quad-0.519426-1.138023$

$\begin{array}{llll}\text { C } & 0.000000 & 0.000000 & -0.593797\end{array}$

C $-1.263815-0.729664-1.065965$

H $\quad-1.181658 \quad-0.682231 \quad-2.162164$

C $1.279565 \quad 2.206069-0.692382$

H $\quad 1.249398 \quad 3.202873-1.138023$

H $\quad \begin{array}{llll}1.312491 & 2.347310 & 0.389420\end{array}$

$\begin{array}{llll}\text { C } & -1.270730 & -2.211170 & -0.692382\end{array}$

H $\quad-2.149070-2.683447-1.138023$

H $\quad-1.376585 \quad-2.310306 \quad 0.389420$

C $1.263815-0.729664-1.065965$

H $\quad 1.181658-0.682231-2.162164$

C $\quad 2.550294 \quad 0.005101 \quad-0.692382$

H $\quad 2.689076-0.037005 \quad 0.389420$

H $3.398468-0.519426-1.138023$

$\begin{array}{llll}\text { C } & 1.270730 & -2.211170 & -0.692382\end{array}$

H $\quad 1.376585 \quad-2.310306 \quad 0.389420$

H $\quad 2.149070-2.683447 \quad-1.138023$

C $-0.000000-2.921726-1.163039$

H $\quad-0.000000-2.960712-2.257274$

H $\quad-0.000000-3.957011-0.817073$

C $\quad 2.530289 \quad 1.460863 \quad-1.163039$

H $\quad 2.564052 \quad 1.480356 \quad-2.257274$

H $3.426872 \quad 1.978506 \quad-0.817073$

I $0.000000 \quad 0.000000 \quad 1.675694$

\section{CCH (B3LYP-D3(BJ)/def2-TZVPP)}

Imaginary frequencies- 0

Electronic Energy (SCF) $=-585.105300620$ a.u

Grimme-D3(BJ) Dispersion energy $=-0.0818806524 \mathrm{a} . \mathrm{u}$

ZPV corrected Energy = -584.767387 a.u.

Enthalpy $=-584.753546$ a.u.

Free Energy $=-584.803758$ a.u.
$\begin{array}{llll}C & 1.267049 & 2.203183 & -0.084831\end{array}$

H $2.151496 \quad 2.692879-0.499329$

H $\quad \begin{array}{llll}\text { H } & 1.351517 & 2.270212 & 1.002963\end{array}$

$\begin{array}{llll}\text { C } & 0.000000 & 2.922442 & -0.550605\end{array}$

H $\quad 0.000000 \quad 2.972371-1.644480$

H $\quad 0.000000 \quad 3.954188-0.193211$

C $1.267148 \quad 0.731588 \quad-0.493854$

H $\quad 1.1911130 .687689-1.587220$

C $\quad-1.267049 \quad 2.203183 \quad-0.084831$

$\begin{array}{llll}\text { H } & -1.351517 & 2.270212 & 1.002963\end{array}$

H $\quad-2.151496 \quad 2.692879 \quad-0.499329$

C $\quad 0.000000 \quad 0.000000 \quad 0.031762$

$\begin{array}{llll}\text { C } & -1.267148 & 0.731588 & -0.493854\end{array}$

H $\quad-1.1911130 .687689-1.587220$

C $2.541537 \quad-0.004295 \quad-0.084831$

H $\quad 3.407850 \quad 0.516810 \quad-0.499329$

H $\quad 2.641820 \quad 0.035342 \quad 1.002963$

$\begin{array}{llll}\text { C } & -2.541537 & -0.004295 & -0.084831\end{array}$

H $\quad-3.407850 \quad 0.516810-0.499329$

$\begin{array}{llll}\text { H } & -2.641820 & 0.035342 & 1.002963\end{array}$

$\begin{array}{llll}\text { C } & -0.000000 & -1.463177 & -0.493854\end{array}$

H $\quad-0.000000-1.375379-1.587220$

C $1.274488-2.198888-0.084831$

H $\quad 1.290303 \quad-2.305554 \quad 1.002963$

H $\quad \begin{array}{llll}1.256354 & -3.209690 & -0.499329\end{array}$

C $-1.274488-2.198888-0.084831$

H $\quad-1.290303-2.305554 \quad 1.002963$

H $\quad-1.256354 \quad-3.209690 \quad-0.499329$

C $-2.530909-1.461221-0.550605$

H $-2.574149-1.486186-1.644480$

H $\quad-3.424427 \quad-1.977094 \quad-0.193211$

C $2.530909-1.461221-0.550605$

H $2.574149-1.486186-1.644480$

H $\quad 3.424427-1.977094 \quad-0.193211$

C $\quad 0.000000 \quad 0.000000 \quad 1.496247$

C $\quad 0.000000 \quad 0.000000 \quad 2.696841$

H $\quad 0.000000 \quad 0.000000 \quad 3.757990$

\section{CN (B3LYP-D3(BJ)/def2-TZVPP)}

Imaginary frequencies- 0

Electronic Energy (SCF) $=-601.211414029$ a.u

Grimme-D3(BJ) Dispersion energy $=-0.0802533317$ a.u

ZPV corrected Energy $=-600.883768$ a.u.

Enthalpy $=-600.870198$ a.u.

Free Energy $=-600.920102$ a.u.

$\begin{array}{llll}C & 1.266181 & 2.203024 & -0.065202\end{array}$

H $\quad 2.151877 \quad 2.696252 \quad-0.471484$

H $\quad 1.348306 \quad 2.262558 \quad 1.023673$

C $0.000000 \quad 2.924723 \quad-0.528438$

H $\quad 0.000000 \quad 2.981673-1.621780$

H $\quad 0.000000 \quad 3.953395 \quad-0.163754$

C $\quad 1.272580 \quad 0.734724-0.487530$

H $\quad 1.2060620 .696320-1.581076$

C $\quad-1.266181 \quad 2.203024-0.065202$

H $\quad-1.348306 \quad 2.262558 \quad 1.023673$

H $\quad-2.151877 \quad 2.696252-0.471484$

$\begin{array}{llll}\text { C } & 0.000000 & 0.000000 & 0.014732\end{array}$

C $-1.272580 \quad 0.734724 \quad-0.487530$

H $\quad-1.2060620 .696320-1.581076$

C $2.540966 \quad-0.004967-0.065202$

H $3.410961 \quad 0.515454 \quad-0.471484$ 


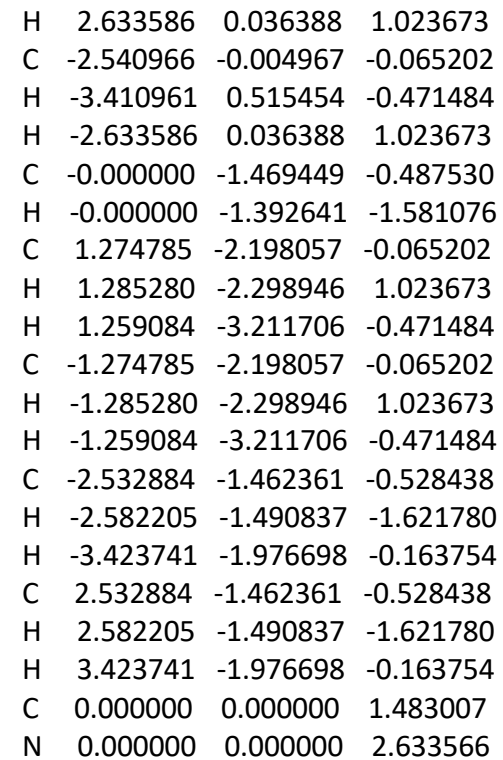

\section{Me (B3LYP-D3(BJ)/def2-TZVPP)}

Imaginary frequencies- 0

Electronic Energy (SCF) $=-548.255817928$ a.u Grimme-D3(BJ) Dispersion energy $=-0.0809579302 \mathrm{a} . \mathrm{u}$ ZPV corrected Energy $=-547.898712$ a.u. Enthalpy $=-547.885574$ a.u.

Free Energy $=-547.934178$ a.u.

\begin{tabular}{|c|c|c|c|}
\hline & 1.2 & 22 & \\
\hline & 2.147748 & 681533 & -0424148 \\
\hline & 1.389913 & 340 & 1.1 \\
\hline & 0.000000 & 2.920422 & 0030 \\
\hline & 0.000000 & 2.953438 & -1.534363 \\
\hline & 0.000000 & 3.958139 & -0.099577 \\
\hline & 1.253934 & .723959 & -0.342629 \\
\hline & 1.164968 & 0.672595 & -1.436074 \\
\hline & -1.271953 & 2.208022 & 0.026813 \\
\hline & -1.389913 & 2.322040 & 1.107558 \\
\hline & -2.147748 & 2.681533 & -0.424148 \\
\hline & 0.000000 & 0.000000 & 0.211457 \\
\hline & -1.253934 & 0.723959 & -0.342629 \\
\hline & -1.164968 & 0.672595 & -1.436074 \\
\hline & 2.548180 & -0.002467 & 0.026813 \\
\hline & 3.396150 & 0.519237 & -0.424148 \\
\hline & 2.705902 & 0.042680 & 1.107558 \\
\hline & -2.548180 & -0.002467 & 0.026813 \\
\hline & -3.396150 & 0.519237 & -0.42414 \\
\hline & -2.705902 & 0.042680 & 1.107558 \\
\hline & -0.000000 & -1.447918 & -0.342629 \\
\hline & -0.000000 & -1.345189 & -1.436074 \\
\hline & 1.276227 & -2.205555 & 0.026813 \\
\hline & 1.315989 & -2.364720 & 1.107558 \\
\hline & 1.248402 & -3.200771 & -0.424148 \\
\hline & -1.276227 & -2.205555 & 0.026813 \\
\hline & -1.315989 & -2.364720 & 1.107558 \\
\hline & -1.248402 & -3.200771 & -0.424148 \\
\hline & -2.529159 & -1.460211 & -0.440030 \\
\hline & -2.557752 & -1.476719 & -1.534363 \\
\hline & -3.427849 & -1.979070 & -0.099577 \\
\hline & 2.529159 & -1.460211 & -0.440030 \\
\hline & 2.55775 & -1.476719 & -1.534 \\
\hline
\end{tabular}

$\begin{array}{lrrr}\text { H } & 3.427849 & -1.979070 & -0.099577 \\ \mathrm{C} & 0.000000 & 0.000000 & 1.750431 \\ \mathrm{H} & 0.000000 & 1.009666 & 2.153613 \\ \mathrm{H} & -0.874396 & -0.504833 & 2.153613 \\ \mathrm{H} & 0.874396 & -0.504833 & 2.153613\end{array}$

\section{Et (B3LYP-D3(BJ)/def2-TZVPP)}

Imaginary frequencies- 0

Electronic Energy (SCF) = -587.578134436 a.u Grimme-D3(BJ) Dispersion energy $=-0.0889375736 \mathrm{a} . \mathrm{u}$ ZPV corrected Energy $=-587.191763$ a.u.

Enthalpy $=-587.177460$ a.u.

Free Energy $=-587.229532$ a.u.

$\begin{array}{llll}C & 0.889744 & 2.342489 & -0.208002\end{array}$

H $1.676955 \quad 2.925451-0.692931$

H $\quad 1.042689 \quad 2.455762 \quad 0.867977$

C $-0.481929 \quad 2.895479-0.604821$

H $\quad-0.538298 \quad 2.923895 \quad-1.697716$

H $\quad-0.592775 \quad 3.926604 \quad-0.262414$

C $1.0156320 .873009-0.612313$

H $\quad 0.764844 \quad 0.832580 \quad-1.681454$

C $\quad-1.634113 \quad 2.037541 \quad-0.074579$

H $\quad-1.736793 \quad 2.182574 \quad 1.003614$

H $\quad-2.577308 \quad 2.377385 \quad-0.510086$

$\begin{array}{llll}\text { C } & -0.064365 & 0.000362 & 0.074433\end{array}$

C $\quad-1.446048 \quad 0.552508-0.395325$

$\begin{array}{llll}\mathrm{H} & -1.434347 & 0.471157 & -1.489658\end{array}$

C $2.437208 \quad 0.310965-0.511301$

H $\quad 3.097238 \quad 0.925707 \quad-1.129502$

H $\quad 2.8184410 .390178 \quad 0.503960$

$\begin{array}{llll}\text { C } & -2.605214 & -0.317173 & 0.095159\end{array}$

H $\quad-3.546757 \quad 0.088574 \quad-0.283828$

H $\quad-2.673011-0.269991 \quad 1.184782$

C $0.052681-1.434732 \quad-0.506249$

H $\quad-0.090810-1.313406-1.588791$

C $1.447350-2.034451-0.335040$

H $\quad 1.666947-2.188997 \quad 0.722521$

H $\quad 1.470867-3.025565-0.796029$

C $-1.073288-2.353901-0.032190$

H $\quad-0.988152-2.538240 \quad 1.041592$

H $\quad-0.964337-3.329780-0.512156$

C $-2.450673-1.770473-0.363251$

H $\quad-2.588720-1.806752 \quad-1.448658$

H $\quad-3.239146-2.390327 \quad 0.068981$

C $2.510909-1.144461-0.983523$

H $\quad 2.360518-1.168812-2.067816$

H $3.509286-1.548190-0.801894$

C $\quad-0.037350 \quad 0.030201 \quad 1.633328$

H $\quad-0.570725 \quad 0.921870 \quad 1.959535$

H $\quad-0.646550 \quad-0.798144 \quad 1.989631$

C $\quad 1.270401 \quad 0.004216 \quad 2.430519$

$\begin{array}{llll}\text { H } & 1.873473 & 0.895518 & 2.268467\end{array}$

H $\quad \begin{array}{llll}\text { H } & 1.027814 & -0.027803 & 3.494617\end{array}$

H $\quad \begin{array}{lll}1.892976 & -0.860717 & 2.216171\end{array}$

\section{3iPr (B3LYP-D3(BJ)/def2-TZVPP)}

Imaginary frequencies- 0

Electronic Energy (SCF) = -626.896484346 a.u Grimme-D3(BJ) Dispersion energy $=-0.0981494338$ a.u ZPV corrected Energy $=-626.481208$ a.u. 
Enthalpy $=-626.465814$ a.u.

Free Energy $=-626.519883$ a.u.

$\begin{array}{llll}C \quad 2.079814 & 1.551819 & -0.187805\end{array}$

H $3.057059 \quad 1.643268-0.669177$

H $\quad 2.270976 \quad 1.530544 \quad 0.885623$

C $\quad 1.221350 \quad 2.772607 \quad-0.531799$

H $\quad 1.211391 \quad 2.894571 \quad-1.619603$

H $\quad 1.669892 \quad 3.680285-0.122329$

$\begin{array}{llll}\text { C } & 1.406083 & 0.257093 & -0.653737\end{array}$

H $1.184996 \quad 0.417714 \quad-1.716550$

C $\quad-0.223942 \quad 2.627078-0.047064$

$\begin{array}{llll}\text { H } & -0.269082 & 2.733807 & 1.039576\end{array}$

H $\quad-0.829571 \quad 3.442484-0.450829$

C $-0.003970 \quad 0.053693 \quad-0.010800$

$\begin{array}{llll}\text { C } & -0.829681 & 1.293762 & -0.487455\end{array}$

H $\quad-0.736879 \quad 1.276525 \quad-1.581014$

$\begin{array}{llll}\text { C } & 2.318832 & -0.977332 & -0.635414\end{array}$

H $3.181436-0.768101-1.274298$

H $\quad 2.723771-1.1608810 .354789$

$\begin{array}{llll}\text { C } & -2.327571 & 1.178179 & -0.212986\end{array}$

$\begin{array}{llll}\text { H } & -2.829524 & 2.056919 & -0.627025\end{array}$

H $\quad-2.528222 \quad 1.201851 \quad 0.857978$

C $-0.617410-1.171442-0.742836$

H $\quad-0.507105-0.919774-1.806734$

C $0.217598 \quad-2.435308-0.543512$

H $\quad 0.283964-2.6910160 .515332$

H $\quad-0.273682-3.280777 \quad-1.032349$

C $-2.125806-1.363823-0.555605$

H $\quad-2.362029-1.726524 \quad 0.440000$

H $\quad-2.452501-2.149720-1.242322$

C $\quad-2.910264-0.081451-0.865102$

H $\quad-2.898753 \quad 0.071928$ - 1.948977

H $\quad-3.958631-0.205872-0.585432$

C $1.612762-2.240000-1.145762$

H $\quad 1.499320-2.166145-2.232023$

H $\quad 2.238970-3.115441-0.960703$

C $0.036852 \quad-0.012082 \quad 1.575322$

$\begin{array}{llll}\text { H } & 0.030659 & 1.030667 & 1.892037\end{array}$

C $1.281511-0.6148292 .252684$

H $\quad 1.388083 \quad-1.679817 \quad 2.053191$

H $\quad 2.209126 \quad-0.125847 \quad 1.977500$

H $\quad \begin{array}{llll}\text { H.170081 } & -0.501844 & 3.332588\end{array}$

C $-1.157503 \quad-0.666596 \quad 2.293223$

H $-1.155448-1.748994 \quad 2.165386$

H $\quad-1.063884-0.473395 \quad 3.363321$

H $\quad-2.126358 \quad-0.294622 \quad 1.983924$

\section{3tBu (B3LYP-D3(BJ)/def2-TZVPP)}

Imaginary frequencies- 0

Electronic Energy (SCF) = -666.213186084 a.u Grimme-D3(BJ) Dispersion energy $=-0.1084817317 \mathrm{a} . \mathrm{u}$ ZPV corrected Energy $=-665.768806$ a.u.

Enthalpy $=-665.752541$ a.u.

Free Energy $=-665.806723$ a.u.

C $\quad-0.006853 \quad 2.582026-0.628990$

H $\quad 0.5000593 .279680-1.301651$

$\begin{array}{llll}\mathrm{H} & 0.084867 & 3.014439 & 0.361223\end{array}$

C $-1.484600 \quad 2.493271-1.036956$

H $\quad-1.538600 \quad 2.415595-2.127381$

H $\quad-2.000920 \quad 3.417862 \quad-0.769852$
C $\quad 0.713390 \quad 1.230798 \quad-0.739598$

H $\quad 0.654193 \quad 0.998770 \quad-1.810221$

C $\quad-2.214474 \quad 1.278527 \quad-0.451491$

H $\quad-2.390010 \quad 1.4063240 .616066$

H $\quad-3.202926 \quad 1.199819-0.912061$

$\begin{array}{llll}\text { C } & 0.000000 & 0.000000 & -0.079999\end{array}$

$\begin{array}{llll}\text { C } & -1.422597 & 0.002415 & -0.739598\end{array}$

H $\quad-1.192057 \quad 0.067163 \quad-1.810221$

C $2.214474 \quad 1.278527 \quad-0.451491$

H $2.6405372 .173906-0.912061$

H $\quad 2.412917 \quad 1.366647 \quad 0.616066$

C $-2.232674-1.296948-0.628990$

H $-3.090316-1.206776-1.301651$

H $\quad-2.653014 \quad-1.433723 \quad 0.361223$

$\begin{array}{llll}\text { C } & 0.709207 & -1.233212 & -0.739598\end{array}$

H $\quad 0.537863 \quad-1.065933-1.810221$

C $2.239527-1.285078-0.628990$

H $\quad 2.568148 \quad-1.580717 \quad 0.361223$

H $\quad 2.590257-2.072904-1.301651$

C $-0.000000-2.557055-0.451491$

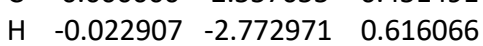

H $\quad 0.562389-3.373725-0.912061$

C $-1.416936-2.532337 \quad-1.036956$

H $\quad-1.322666-2.540264 \quad-2.127381$

H $-1.959496-3.441779-0.769852$

C $2.901536 \quad 0.039066-1.036956$

H $\quad 2.861266 \quad 0.124670 \quad-2.127381$

H $\quad 3.960416 \quad 0.023917 \quad-0.769852$

C $\quad 0.000000 \quad 0.000000 \quad 1.544818$

$\begin{array}{llll}\text { C } & 1.230920 & -0.685798 & 2.176580\end{array}$

H $\quad 1.291030-1.742892 \quad 1.934015$

H $\quad 2.169774-0.215555 \quad 1.901455$

H $\quad \begin{array}{llll}\text { H } & 1.140978 & -0.611831 & 3.261498\end{array}$

C $\quad-1.209378-0.723109 \quad 2.176580$

H $\quad-1.271563 \quad-1.771302 \quad 1.901455$

H $\quad-1.100350-0.6822013 .261498$

H $\quad-2.154903 \quad-0.246619 \quad 1.934015$

C $\quad-0.021542 \quad 1.408907 \quad 2.176580$

H $\quad-0.040628 \quad 1.294032 \quad 3.261498$

H $\quad 0.863874 \quad 1.989510 \quad 1.934015$

H $\quad-0.898211 \quad 1.986857 \quad 1.901455$

\section{Ph (B3LYP-D3(BJ)/def2-TZVPP)}

Imaginary frequencies- 0

Electronic Energy (SCF) $=-740.063803017$ a.u

Grimme-D3(BJ) Dispersion energy $=-0.1078165323$ a.u ZPV corrected Energy = -739.652434 a.u.

Enthalpy $=-739.636231$ a.u.

Free Energy $=-739.693412$ a.u.

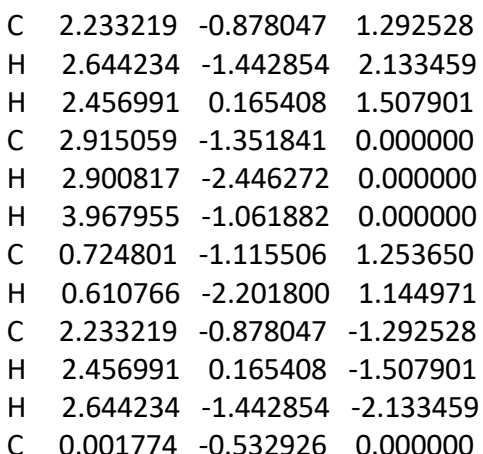

$\begin{array}{llll}\text { C } & 2.233219 & -0.878047 & 1.292528\end{array}$

H $\quad 2.644234 \quad-1.4428542 .133459$

H $\quad 2.456991 \quad 0.165408 \quad 1.507901$

C $2.915059-1.351841 \quad 0.000000$

$\begin{array}{llll}\text { H } & 2.900817 & -2.446272 & 0.000000\end{array}$

H $3.967955-1.0618820 .000000$

C $\quad 0.724801 \quad-1.115506 \quad 1.253650$

H $\quad 0.610766 \quad-2.201800 \quad 1.144971$

C $2.233219-0.878047-1.292528$

H $\quad 2.4569910 .165408-1.507901$

C $0.001774 \quad-0.532926 \quad 0.000000$ 
$\begin{array}{llll}C & 0.724801 & -1.115506 & -1.253650\end{array}$

H $0.610766 \quad-2.201800 \quad-1.144971$

$\begin{array}{llll}\text { C } & -0.008485 & -0.733398 & 2.536901\end{array}$

H $\quad 0.552971 \quad-1.089819 \quad 3.404086$

$\begin{array}{llll}\text { H } & -0.063268 & 0.354915 & 2.623461\end{array}$

C $-0.008485-0.733398 \quad-2.536901$

H $\quad 0.552971-1.089819-3.404086$

$\begin{array}{llll}\text { H } & -0.063268 & 0.354915 & -2.623461\end{array}$

C $-1.414191 \quad-1.198636 \quad 0.000000$

H $\quad-1.183499-2.270403 \quad 0.000000$

C $-2.215096-0.973755 \quad 1.291565$

$\begin{array}{llll}\text { H } & -2.550958 & 0.057068 & 1.382379\end{array}$

H $\quad-3.121550 \quad-1.583378 \quad 1.245659$

C $-2.215096-0.973755-1.291565$

H $\quad-2.550958 \quad 0.057068$ - 1.382379

H $\quad-3.121550 \quad-1.583378 \quad-1.245659$

C $-1.412525-1.342890-2.543736$

H $-1.313526-2.431882-2.596425$

H $\quad-1.960086-1.037878-3.437869$

$\begin{array}{llll}\text { C } & -1.412525 & -1.342890 & 2.543736\end{array}$

H $\quad-1.313526 \quad-2.431882 \quad 2.596425$

H $\quad-1.960086-1.037878 \quad 3.437869$

C $-0.019562 \quad 1.0131180 .000000$

C $\quad-1.204122 \quad 1.760115 \quad 0.000000$

C $\quad 1.161030 \quad 1.772837 \quad 0.000000$

C $\quad-1.218486 \quad 3.149730 \quad 0.000000$

H $\quad-2.156572 \quad 1.268450 \quad 0.000000$

C $\quad 1.159202 \quad 3.161950 \quad 0.000000$

H $\quad 2.119077 \quad 1.293901 \quad 0.000000$

C $\quad-0.033657 \quad 3.868274 \quad 0.000000$

H $\quad-2.169679 \quad 3.665811 \quad 0.000000$

H $\quad 2.104629 \quad 3.6885110 .000000$

H $\quad-0.039132 \quad 4.9497490 .000000$

\section{CF 3 (B3LYP-D3(BJ)/def2-TZVPP)}

Imaginary frequencies- 0

Electronic Energy (SCF) = -846.111433889 a.u

Grimme-D3(BJ) Dispersion energy $=-0.0825355103$ a.u ZPV corrected Energy $=-845.776573$ a.u.

Enthalpy $=-845.761598$ a.u.

Free Energy $=-845.814945$ a.u.

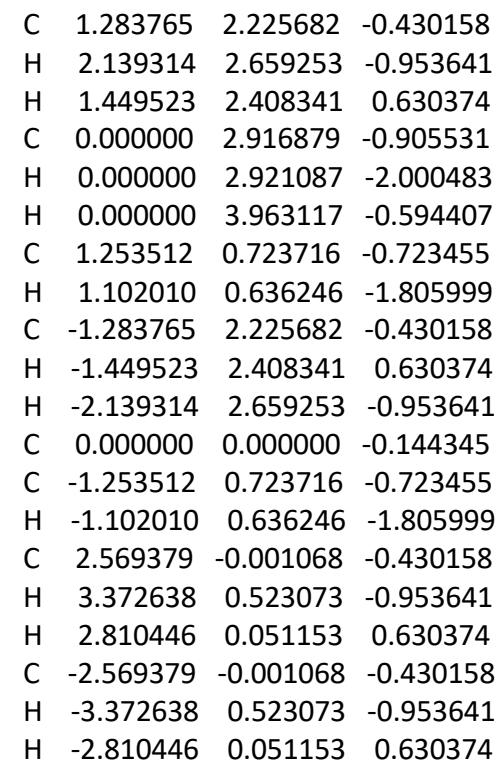

C $\quad-0.000000-1.447431-0.723455$

H $\quad-0.000000-1.272491 \quad-1.805999$

C $1.285614 \quad-2.224614-0.430158$

H $\quad \begin{array}{llll}1.360923 & -2.459494 & 0.630374\end{array}$

H $\quad 1.233324-3.182327 \quad-0.953641$

C $\quad-1.285614-2.224614 \quad-0.430158$

$\begin{array}{llll}\text { H } & -1.360923 & -2.459494 & 0.630374\end{array}$

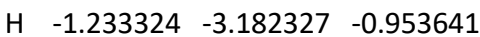

C $-2.526091-1.458440-0.905531$

H $\quad-2.529735-1.460543-2.000483$

H $\quad-3.432160-1.981559-0.594407$

C $2.526091-1.458440-0.905531$

H $2.529735-1.460543-2.000483$

H $3.432160 \quad-1.981559-0.594407$

C $\quad 0.000000 \quad 0.000000 \quad 1.401249$

$\begin{array}{llll}\text { F } & 0.000000 & 1.241349 & 1.941048\end{array}$

F $\quad-1.075039-0.620674 \quad 1.941048$

F $\quad \begin{array}{lll}1.075039 & -0.620674 & 1.941048\end{array}$

\section{CCl 3 (B3LYP-D3(BJ)/def2-TZVPP)}

Imaginary frequencies- 0

Electronic Energy (SCF) = -1927.08487749 a.u

Grimme-D3(BJ) Dispersion energy $=-0.1028249181 \mathrm{a} . \mathrm{u}$ ZPV corrected Energy $=-1926.755402$ a.u.

Enthalpy $=-1926.738967$ a.u.

Free Energy = -1926.796476 a.u.

C $\quad-0.000000 \quad 2.587275 \quad-0.970380$

H $\quad 0.497874 \quad 3.275488$ - 1.658588

H $\quad 0.124753 \quad 3.0146170 .017965$

C $-1.486350 \quad 2.505339-1.356606$

H $\quad-1.549872 \quad 2.466650 \quad-2.448664$

H $\quad-1.997103 \quad 3.421359-1.054352$

C $0.719346 \quad 1.239814 \quad-1.090240$

H $\quad 0.644063 \quad 0.991884-2.154558$

C $\quad-2.230041 \quad 1.276193 \quad-0.813693$

$\begin{array}{llll}\text { H } & -2.455769 & 1.378171 & 0.245047\end{array}$

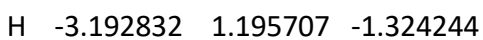

C $\quad 0.000000 \quad 0.000000 \quad-0.445202$

C $-1.433383 \quad 0.003065-1.090240$

$\begin{array}{llll}\text { H } & -1.181028 & 0.061833 & -2.154558\end{array}$

C $2.220236 \quad 1.293176-0.813693$

H $2.631929 \quad 2.167220 \quad-1.324244$

H $2.421416 \quad 1.437673 \quad 0.245047$

C $-2.240646-1.293637-0.970380$

H $\quad-3.085593 \quad-1.206572 \quad-1.658588$

H $\quad-2.673111-1.399270 \quad 0.017965$

C $0.714037-1.242879-1.090240$

H $\quad 0.536965 \quad-1.053716 \quad-2.154558$

$\begin{array}{llll}\text { C } & 2.240646 & -1.293637 & -0.970380\end{array}$

$\begin{array}{llll}\text { H } & 2.548359 & -1.615347 & 0.017965\end{array}$

H $\quad 2.587719 \quad-2.068916-1.658588$

C $0.009805-2.569369-0.813693$

$\begin{array}{llll}\text { H } & 0.034353 & -2.815844 & 0.245047\end{array}$

H $\quad 0.560903 \quad-3.362927 \quad-1.324244$

$\begin{array}{llll}\text { C } & -1.426512 & -2.539887 & -1.356606\end{array}$

H $-1.361246-2.575554 \quad-2.448664$

H $\quad-1.964433 \quad-3.440221 \quad-1.054352$

C 2.9128620 .034548 -1.356606

$\begin{array}{llll}\text { H } & 2.911118 & 0.108904 & -2.448664\end{array}$

H $3.9615350 .018862 \quad-1.054352$

$\begin{array}{llll}\text { C } & 0.000000 & 0.000000 & 1.142778\end{array}$ 
Cl $\quad-0.502333 \quad 1.564563 \quad 1.903986$

Cl $\quad 1.606118-0.347248 \quad 1.903986$

Cl $\quad-1.103785-1.217315 \quad 1.903986$

\section{$3 \mathrm{CBr}_{3}$ (B3LYP-D3(BJ)/def2-TZVPP)}

Imaginary frequencies- 0

Electronic Energy (SCF) $=-8268.94190400$ a.u Grimme-D3(BJ) Dispersion energy $=-0.1096221122$ a.u ZPV corrected Energy $=-8268.614579$ a.u. Enthalpy $=-8268.597080$ a.u.

Free Energy $=-8268.658388$ a.u.

$\begin{array}{llll}\text { C } \quad 2.586350 & 0.010855 & 1.564757\end{array}$

H $\quad 3.243818-0.473725 \quad 2.291586$

H $\quad 3.057704 \quad-0.133746 \quad 0.600522$

C $2.494162 \quad 1.506353 \quad 1.915940$

H $\quad 2.447962 \quad 1.597490 \quad 3.005564$

H $\quad 3.410310 \quad 2.012480 \quad 1.606617$

C $1.238926-0.715295 \quad 1.641613$

H $\quad 0.968811 \quad-0.653659 \quad 2.700871$

C $\quad 1.265030 \quad 2.232120 \quad 1.349352$

H $\quad 1.369441 \quad 2.432643 \quad 0.285451$

H $\quad 1.177968 \quad 3.206882 \quad 1.835326$

C $0.000000 \quad 0.000000 \quad 0.983938$

C $\quad-0.000000 \quad 1.430589 \quad 1.641613$

H $\quad 0.081679 \quad 1.165845 \quad 2.700871$

C $1.300558-2.211608 \quad 1.349352$

H $\quad 2.188257-2.623592 \quad 1.835326$

H $\quad \begin{array}{llll}1.422010 & -2.402293 & 0.285451\end{array}$

C $\quad-1.302576 \quad 2.234417 \quad 1.564757$

H $\quad-1.211652 \quad 3.046091 \quad 2.291586$

$\begin{array}{llll}\text { H } & -1.413025 & 2.714923 & 0.600522\end{array}$

C $-1.238926-0.715295 \quad 1.641613$

H $\quad-1.050491 \quad-0.512186 \quad 2.700871$

C $-1.283774-2.245272 \quad 1.564757$

$\begin{array}{llll}\text { H } & -1.644680 & -2.581177 & 0.600522\end{array}$

$\begin{array}{llll}\text { H } & -2.032167 & -2.572367 & 2.291586\end{array}$

C $-2.565588-0.020512 \quad 1.349352$

$\begin{array}{llll}\text { H } & -2.791451 & -0.030351 & 0.285451\end{array}$

H $\quad-3.366226-0.583291 \quad 1.835326$

C $\quad-2.551621 \quad 1.406832 \quad 1.915940$

$\begin{array}{llll}\text { H } & -2.607448 & 1.321252 & 3.005564\end{array}$

H $\quad-3.448014 \quad 1.947175 \quad 1.606617$

C $0.057459-2.913184 \quad 1.915940$

H $\quad 0.159486 \quad-2.918742 \quad 3.005564$

H $\quad 0.037704 \quad-3.959655 \quad 1.606617$

C $\quad 0.000000 \quad 0.000000 \quad-0.604942$

$\begin{array}{lllll}\mathrm{Br} & -1.250441 & 1.284166 & -1.483254\end{array}$

$\begin{array}{lllll}\mathrm{Br} & -0.486900 & -1.724997 & -1.483254\end{array}$

$\begin{array}{llll}\mathrm{Br} & 1.737341 & 0.440831 & -1.483254\end{array}$

\section{Cl 3 (B3LYP-D3(BJ)/def2-TZVPP)}

Imaginary frequencies- 0

Electronic Energy (SCF) = -1439.79091136 a.u

Grimme-D3(BJ) Dispersion energy $=-0.1200334731 \mathrm{a} . \mathrm{u}$

ZPV corrected Energy = -1439.465013 a.u.

Enthalpy $=-1439.446857$ a.u.

Free Energy = -1439.511084 a.u.

C $\quad-2.426867 \quad 0.818979 \quad 1.743344$

H $\quad-3.018068 \quad 1.611564 \quad 2.208190$ $\begin{array}{llll}\text { H } & -2.624767 & 0.883597 & 0.675188\end{array}$

C $-2.866395 \quad-0.532766 \quad 2.324112$

H $\quad-2.909517 \quad-0.416229 \quad 3.411237$

H $\quad-3.881127 \quad-0.773571 \quad 2.002438$

C $\quad-0.953159 \quad 1.061125 \quad 2.049729$

$\begin{array}{llll}\text { H } & -0.844617 & 0.791738 & 3.105092\end{array}$

C $\quad-1.931761 \quad-1.713324 \quad 2.003169$

H $\quad-2.197347 \quad-2.168527 \quad 1.057629$

$\begin{array}{llll}\text { H } & -2.087139 & -2.491470 & 2.755448\end{array}$

C $\quad 0.000000 \quad 0.000000 \quad 1.379260$

C $-0.442381-1.356022 \quad 2.049729$

H $\quad-0.263357 \quad-1.127329 \quad 3.105092$

C $-0.517902 \quad 2.529616 \quad 2.003169$

H $\quad-1.114107 \quad 3.053251 \quad 2.755448$

H $\quad-0.779326 \quad 2.987222 \quad 1.057629$

$\begin{array}{llll}\text { C } & 0.504177 & -2.511218 & 1.743344\end{array}$

H $\quad 0.113379-3.419505 \quad 2.208190$

H $\quad 0.547166 \quad-2.714913 \quad 0.675188$

C $\quad 1.395541 \quad 0.294898 \quad 2.049729$

H $\quad 1.107974 \quad 0.335591 \quad 3.105092$

C $\quad 1.922690 \quad 1.692239 \quad 1.743344$

H $2.077601 \quad 1.8313160 .675188$

H $\quad 2.904689 \quad 1.807942 \quad 2.208190$

C $2.449663 \quad-0.816292 \quad 2.003169$

H $\quad 2.976673 \quad-0.818695 \quad 1.057629$

H $\quad 3.201246 \quad-0.561781 \quad 2.755448$

$\begin{array}{llll}\text { C } & 1.894586 & -2.215988 & 2.324112\end{array}$

H $\quad 1.815224 \quad-2.311601 \quad 3.411237$

H $\quad 2.610495 \quad-2.974369 \quad 2.002438$

C $\quad 0.971809 \quad 2.748754 \quad 2.324112$

H $\quad 1.094293 \quad 2.727830 \quad 3.411237$

H $\quad 1.270631 \quad 3.747940 \quad 2.002438$

$\begin{array}{llll}\text { C } & 0.000000 & 0.000000 & -0.213097\end{array}$

I $\quad-0.000000 \quad 1.979363 \quad-1.250371$

| $1.714179-0.989681-1.250371$

| $\begin{array}{llll}-1.714179 & -0.989681 & -1.250371\end{array}$

\section{SiMe 3 (B3LYP-D3(BJ)/def2-TZVPP)}

Imaginary frequencies- 0

Electronic Energy (SCF) = -917.684539139 a.u

Grimme-D3(BJ) Dispersion energy $=-0.1121316462$ a.u ZPV corrected Energy = -917.252359 a.u.

Enthalpy $=-917.233717$ a.u.

Free Energy $=-917.293288$ a.u.

C $\quad 1.276723 \quad 2.229342 \quad-0.773795$

H $\quad 2.100318 \quad 2.647911 \quad-1.358625$

H $\quad 1.503440 \quad 2.455506 \quad 0.263783$

C $\quad-0.032340 \quad 2.913174-1.182337$

H $\quad-0.100952 \quad 2.920133 \quad-2.274669$

H $\quad-0.021354 \quad 3.958863 \quad-0.867388$

C $1.241843 \quad 0.716979-1.003007$

$\begin{array}{llll}\text { H } & 1.127291 & 0.588796 & -2.089734\end{array}$

$\begin{array}{llll}\text { C } & -1.272424 & 2.199962 & -0.636348\end{array}$

$\begin{array}{llll}\mathrm{H} & -1.337573 & 2.322797 & 0.447768\end{array}$

$\begin{array}{llll}\text { H } & -2.172921 & 2.662540 & -1.048568\end{array}$

C $0.000000 \quad 0.000000-0.399028$

$\begin{array}{llll}\text { C } & -1.241843 & 0.716979 & -1.003007\end{array}$

H $\quad-1.073558 \quad 0.681865 \quad-2.089734$

C $2.5414350 .001970-0.636348$

H $\quad 3.3922880 .550535 \quad-1.048568$

H $\quad 2.680388 \quad-0.003026 \quad 0.447768$ 
$\begin{array}{llll}\text { C } & -2.569028 & -0.008996 & -0.773795\end{array}$

H $\quad-3.343318 \quad 0.494973 \quad-1.358625$

$\begin{array}{llll}\text { H } & -2.878250 & 0.074265 & 0.263783\end{array}$

C $-0.000000-1.433957-1.003007$

H $\quad-0.053734-1.270661-2.089734$

C $1.292305 \quad-2.220346-0.773795$

H $\quad 1.374810-2.529770 \quad 0.263783$

H $1.242999-3.142885-1.358625$

$\begin{array}{llll}\text { C } & -1.269011 & -2.201933 & -0.636348\end{array}$

H $\quad-1.342814-2.3197710 .447768$

H $-1.219367-3.213075-1.048568$

$\begin{array}{llll}\text { C } & -2.506712 & -1.484594 & -1.182337\end{array}$

H $\quad-2.478434-1.547493-2.274669$

H $-3.417799-1.997925-0.867388$

C $2.539053 \quad-1.428579-1.182337$

H $2.579385-1.372640-2.274669$

H $\quad 3.439153 \quad-1.960938$ - 0.867388

C $0.891251 \quad-1.459025 \quad 2.374979$

H $\quad 0.430201-2.421471 \quad 2.156972$

H $\quad 1.947729-1.522294 \quad 2.118927$

H $\quad 0.825174 \quad-1.304591 \quad 3.455010$

C $\quad-1.709178-0.042333 \quad 2.374979$

$\begin{array}{llll}\text { H } & -2.292210 & -0.925636 & 2.118927\end{array}$

H $-1.542396-0.062326 \quad 3.455010$

$\begin{array}{llll}H & -2.312156 & 0.838170 & 2.156972\end{array}$

C $\quad 0.817927 \quad 1.501358 \quad 2.374979$

H $\quad 0.717222 \quad 1.366917 \quad 3.455010$

H $1.881955 \quad 1.583300 \quad 2.156972$

H $\quad 0.344480 \quad 2.447930 \quad 2.118927$

Si $\quad 0.000000 \quad 0.000000 \quad 1.568065$

Geometries of fragments for dispersion computations:

2H from 2F:

Electronic Energy (SCF) $=-390.918424562$ a.u Grimme-D3(BJ) Dispersion energy $=-0.0528407553 \mathrm{a} . \mathrm{u}$

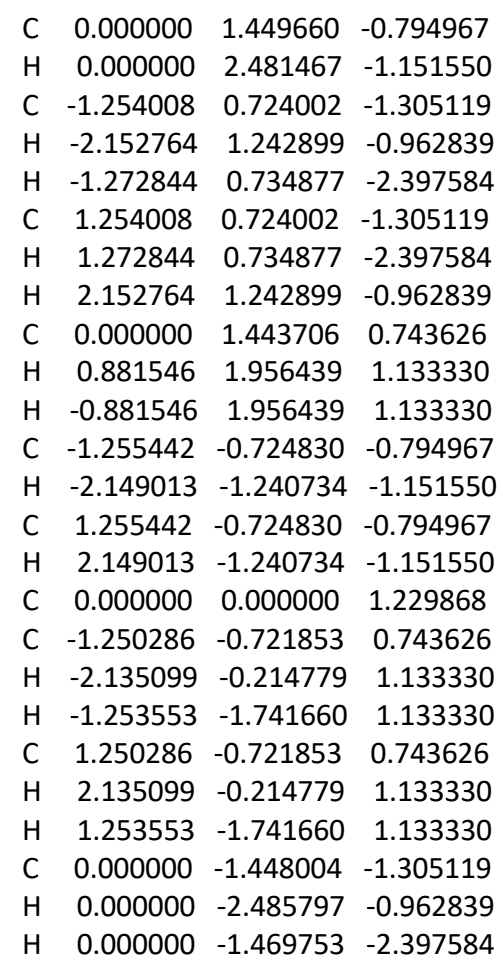

$\begin{array}{llll}H & 0.000000 & 0.000000 & 2.319868\end{array}$

2H from 2Cl:

Electronic Energy (SCF) = -390.918655035 a.u Grimme-D3(BJ) Dispersion energy $=-0.0528100360 \mathrm{a} . \mathrm{u}$

C $\quad 0.000000 \quad 1.447722 \quad-1.103858$

H $\quad 0.000000 \quad 2.481199-1.456106$

C $-1.253982 \quad 0.723987-1.614081$

H $\quad-2.152412 \quad 1.242696-1.270583$

H $\quad-1.273742 \quad 0.735395 \quad-2.706538$

C $1.2539820 .723987 \quad-1.614081$

H $\quad 1.273742 \quad 0.735395-2.706538$

H $\quad 2.152412 \quad 1.242696-1.270583$

C $\quad 0.000000 \quad 1.4460520 .435463$

H $\quad 0.880025 \quad 1.9638760 .819588$

$\begin{array}{llll}\text { H } & -0.880025 & 1.963876 & 0.819588\end{array}$

C $-1.253764-0.723861-1.103858$

H $\quad-2.148782-1.240600-1.456106$

C $1.253764-0.723861-1.103858$

H $\quad 2.148782-1.240600-1.456106$

$\begin{array}{llll}\text { C } & 0.000000 & 0.000000 & 0.926887\end{array}$

C $-1.252318-0.723026 \quad 0.435463$

H $\quad-2.140779-0.219814 \quad 0.819588$

H $\quad-1.260754 \quad-1.7440620 .819588$

C $1.252318-0.723026 \quad 0.435463$

H $\quad 2.140779-0.219814 \quad 0.819588$

H $\quad 1.260754 \quad-1.7440620 .819588$

C $0.000000-1.447974-1.614081$

H $\quad 0.000000-2.485391 \quad-1.270583$

H $\quad 0.000000-1.470791-2.706538$

$\begin{array}{llll}H & 0.000000 & 0.000000 & 2.016887\end{array}$

2H from 2Br:

Electronic Energy (SCF) = -390.918432442 a.u Grimme-D3(BJ) Dispersion energy $=-0.0528131851 \mathrm{a} . \mathrm{u}$

C $\quad 0.000000 \quad 1.447374 \quad-1.609180$

H $\quad 0.000000 \quad 2.481450 \quad-1.959731$

C $\quad-1.254108 \quad 0.724060-2.118839$

H $\quad-2.152335 \quad 1.242651-1.774689$

H $\quad-1.274574 \quad 0.735876-3.211284$

C $1.254108 \quad 0.724060 \quad-2.118839$

H $\quad 1.274574 \quad 0.735876-3.211284$

H $\quad 2.152335 \quad 1.242651-1.774689$

$\begin{array}{llll}\text { C } & 0.000000 & 1.446599 & -0.068037\end{array}$

H $\quad 0.879930 \quad 1.966241 \quad 0.313001$

$\begin{array}{llll}H & -0.879930 & 1.966241 & 0.313001\end{array}$

C $-1.253463-0.723687-1.609180$

H $\quad-2.148999-1.240725-1.959731$

C $1.253463-0.723687-1.609180$

H $\quad 2.148999-1.240725-1.959731$

C $\quad 0.000000 \quad 0.000000 \quad 0.416303$

C $-1.252791-0.723299-0.068037$

H $\quad-2.142780 \quad-0.2210790 .313001$

H $\quad-1.262850-1.745162 \quad 0.313001$

$\begin{array}{llll}\text { C } & 1.252791 & -0.723299 & -0.068037\end{array}$

H $\quad 2.142780 \quad-0.2210790 .313001$

H $\quad \begin{array}{llll}\text { H.262850 } & -1.745162 & 0.313001\end{array}$

C $\quad 0.000000-1.448119-2.118839$

H $\quad 0.000000 \quad-2.485303 \quad-1.774689$ 
$\begin{array}{llll}H & 0.000000 & -1.471752 & -3.211284\end{array}$

H $\quad 0.000000 \quad 0.000000 \quad 1.506303$

2H from 2l:

Electronic Energy (SCF) = -390.918459934 a.u

Grimme-D3(BJ) Dispersion energy $=-0.0527970809 \mathrm{a} . \mathrm{u}$

C $\quad 0.000000 \quad 1.446916 \quad-2.037112$

$\begin{array}{llll}\text { H } & 0.000000 & 2.481460 & -2.386679\end{array}$

C $\quad-1.254142 \quad 0.724079-2.546280$

H $\quad-2.152188 \quad 1.242566 \quad-2.201384$

H $\quad-1.275431 \quad 0.736371-3.638769$

C $1.2541420 .724079-2.546280$

H $\quad \begin{array}{llll}\text { H.275431 } & 0.736371 & -3.638769\end{array}$

H $\quad 2.152188 \quad 1.242566 \quad-2.201384$

C $\quad 0.000000 \quad 1.447265 \quad-0.494743$

H $\quad 0.879567 \quad 1.970379-0.117752$

H $\quad-0.879567 \quad 1.970379-0.117752$

C $-1.253066-0.723458-2.037112$

$\begin{array}{llll}\text { H } & -2.149007 & -1.240730 & -2.386679\end{array}$

C $1.253066-0.723458-2.037112$

H $\quad 2.149007 \quad-1.240730 \quad-2.386679$

C $0.000000 \quad 0.000000-0.009752$

C $-1.253368-0.723633-0.494743$

H $\quad-2.146182-0.223462-0.117752$

H $-1.266614-1.746917-0.117752$

$\begin{array}{llll}\text { C } & 1.253368 & -0.723633 & -0.494743\end{array}$

H $\quad 2.146182-0.223462-0.117752$

H $\quad 1.266614-1.746917 \quad-0.117752$

C $0.000000-1.448159-2.546280$

H $\quad 0.000000 \quad-2.485132 \quad-2.201384$

H $\quad 0.000000 \quad-1.472741-3.638769$

H $\quad 0.000000 \quad 0.000000 \quad 1.080248$

2H from $2 \mathrm{CCH}$ :

Electronic Energy (SCF) = -390.918775401 a.u Grimme-D3(BJ) Dispersion energy $=-0.0526667265$ a.u

C $\quad 0.000000 \quad 1.447947 \quad-1.037045$

$\begin{array}{llll}\text { H } & 0.000000 & 2.479699 & -1.395645\end{array}$

C $\quad-1.2534190 .723662-1.547811$

H $\quad-2.152224 \quad 1.242587 \quad-1.204866$

$\begin{array}{llll}\mathrm{H} & -1.272747 & 0.734821 & -2.640547\end{array}$

C $\quad 1.253419 \quad 0.723662 \quad-1.547811$

H $\quad 1.272747 \quad 0.734821 \quad-2.640547$

H $\quad 2.152224 \quad 1.242587 \quad-1.204866$

C $\quad 0.000000 \quad 1.452455 \quad 0.496700$

H $\quad 0.878739 \quad 1.975952 \quad 0.878259$

H $\quad-0.878739 \quad 1.9759520 .878259$

C $-1.253959-0.723973-1.037045$

H $-2.147483-1.239850-1.395645$

C $1.253959-0.723973-1.037045$

H $\quad 2.147483-1.239850-1.395645$

C $\quad 0.000000 \quad 0.000000 \quad 1.032937$

C $-1.257863 \quad-0.726228 \quad 0.496700$

$\begin{array}{llll}\text { H } & -2.150594 & -0.226966 & 0.878259\end{array}$

H $\quad-1.271856 \quad-1.748986 \quad 0.878259$

C $1.257863 \quad-0.7262280 .496700$

$\begin{array}{llll}\text { H } & 2.150594 & -0.226966 & 0.878259\end{array}$

H $\quad 1.271856-1.7489860 .878259$

C $0.000000-1.447324-1.547811$
H $\quad 0.000000-2.485174-1.204866$

H $\quad 0.000000-1.469641 \quad-2.640547$

H $\quad 0.000000 \quad 0.000000 \quad 2.122937$

2H from 2CN:

Electronic Energy (SCF) = -390.918879068 a.u Grimme-D3(BJ) Dispersion energy $=-0.0526707733 \mathrm{a} . \mathrm{u}$

$\begin{array}{llll}\text { C } & 0.000000 & 1.448314 & -1.019249\end{array}$

H $\quad 0.000000 \quad 2.480049-1.376366$

C $\quad-1.253994 \quad 0.723994-1.528817$

H $\quad-2.152739 \quad 1.242885-1.186300$

H $\quad-1.273170 \quad 0.735065-2.621133$

C $\quad 1.253994 \quad 0.723994-1.528817$

H $\quad 1.273170 \quad 0.735065-2.621133$

H $\quad 2.152739 \quad 1.242885-1.186300$

C $\quad 0.000000 \quad 1.456113 \quad 0.514605$

H $\quad 0.879074 \quad 1.978516 \quad 0.895849$

H $\quad-0.879074 \quad 1.978516 \quad 0.895849$

C $-1.254277-0.724157-1.019249$

H $\quad-2.147786-1.240025-1.376366$

C $1.254277-0.724157-1.019249$

H $\quad 2.147786-1.240025 \quad-1.376366$

C $\quad 0.000000 \quad 0.000000 \quad 1.038610$

C $-1.261031 \quad-0.728057 \quad 0.514605$

H $\quad-2.152982-0.227957 \quad 0.895849$

H $\quad-1.273908 \quad-1.750559 \quad 0.895849$

C $1.261031-0.728057 \quad 0.514605$

H $\quad 2.152982-0.227957 \quad 0.895849$

H $\quad \begin{array}{llll}1.273908 & -1.750559 & 0.895849\end{array}$

C $0.000000 \quad-1.447988-1.528817$

H $\quad 0.000000 \quad-2.485769-1.186300$

H $\quad 0.000000 \quad-1.470130-2.621133$

H $\quad 0.000000 \quad 0.000000 \quad 2.128610$

\section{H from 2Me:}

Electronic Energy (SCF) = -390.918812927 a.u Grimme-D3(BJ) Dispersion energy $=-0.0527174366$ a.u

C $\quad 0.000000 \quad 1.448171 \quad-0.827379$

H $\quad 0.000000 \quad 2.479735-1.187400$

C $-1.252952 \quad 0.723392-1.339051$

H $\quad-2.152066 \quad 1.242496 \quad-0.996342$

H $\quad-1.272476 \quad 0.734664-2.432055$

C $1.252952 \quad 0.723392 \quad-1.339051$

H $\quad 1.272476 \quad 0.734664 \quad-2.432055$

H $\quad 2.152066 \quad 1.242496-0.996342$

$\begin{array}{llll}\text { C } & 0.000000 & 1.442119 & 0.707081\end{array}$

H $\quad 0.878965 \quad 1.9729411 .084520$

H $\quad-0.878965 \quad 1.972941 \quad 1.084520$

C $-1.254153-0.724086-0.827379$

H $\quad-2.147513 \quad-1.239867-1.187400$

C $1.254153-0.724086-0.827379$

H $\quad 2.147513 \quad-1.239867 \quad-1.187400$

C $\quad 0.000000 \quad 0.000000 \quad 1.247692$

$\begin{array}{llll}\text { C } & -1.248911 & -0.721059 & 0.707081\end{array}$

H $\quad-2.148100 \quad-0.225265 \quad 1.084520$

H $-1.269135-1.747676 \quad 1.084520$

C $1.248911-0.7210590 .707081$

H $\quad 2.148100-0.225265 \quad 1.084520$

H $\quad 1.269135-1.747676 \quad 1.084520$ 
$\begin{array}{llll}\text { C } & 0.000000 & -1.446784 & -1.339051\end{array}$

H $\quad 0.000000 \quad-2.484992-0.996342$

H $\quad 0.000000 \quad-1.469328-2.432055$

$\begin{array}{llll}H & 0.000000 & 0.000000 & 2.337692\end{array}$

2H from 2Et:

Electronic Energy (SCF) = -390.918524837 a.u Grimme-D3(BJ) Dispersion energy $=-0.0527209521 \mathrm{a} . \mathrm{u}$

C $\quad-0.987522-0.832103-1.254132$

H $-1.228121-1.413954-2.147124$

C $\quad-1.799517 \quad 0.470077 \quad-1.253197$

H $\quad-1.576425 \quad 1.051415 \quad-2.151883$

H $\quad-2.869308 \quad 0.245590 \quad-1.272957$

C $\quad-1.331500 \quad-1.647975 \quad 0.000001$

H $\quad-2.393940 \quad-1.905596 \quad 0.000001$

$\begin{array}{llll}\mathrm{H} & -0.773117 & -2.588009 & 0.000002\end{array}$

C $0.512135 \quad-0.504065-1.250153$

H $\quad 1.086621 \quad-1.432323 \quad-1.278563$

H $\quad 0.775390 \quad 0.062302-2.148705$

C $\quad-1.454329 \quad 1.286047 \quad-0.000001$

$\begin{array}{lllll}\text { H } & -2.023990 & 2.218459 & -0.000002\end{array}$

C $-0.987522 \quad-0.832102 \quad 1.254133$

H $\quad-1.228122 \quad-1.413951 \quad 2.147126$

C $0.889508 \quad 0.313840 \quad 0.000000$

C $0.045595 \quad 1.603893 \quad-0.000001$

H $\quad 0.301046 \quad 2.203548 \quad-0.878683$

H $\quad 0.301046 \quad 2.203550 \quad 0.878680$

C $0.512135-0.504063 \quad 1.250154$

H $\quad 1.086621 \quad-1.432321 \quad 1.278565$

H $\quad 0.7753890 .062304 \quad 2.148706$

C $\quad-1.799518 \quad 0.470079 \quad 1.253196$

H $\quad-1.576425 \quad 1.051418 \quad 2.151881$

H $\quad-2.8693090 .245592 \quad 1.272955$

H $\quad 1.9447920 .586743 \quad 0.000000$

2H from 2iPr:

Electronic Energy (SCF) = -390.918128459 a.u Grimme-D3(BJ) Dispersion energy $=-0.0527214568$ a.u

$\begin{array}{lrrr}\text { C } & -1.692727 & -0.947167 & 0.000000 \\ \text { H } & -2.784484 & -0.992526 & 0.000000 \\ \text { C } & -1.148736 & -1.648977 & 1.251324 \\ \text { H } & -1.543487 & -1.170077 & 2.151471 \\ \text { H } & -1.478631 & -2.691092 & 1.270670 \\ \text { C } & -1.148736 & -1.648977 & -1.251324 \\ \text { H } & -1.478631 & -2.691092 & -1.270670 \\ \text { H } & -1.543487 & -1.170077 & -2.151471 \\ \text { C } & -1.250329 & 0.524477 & 0.000000 \\ \text { H } & -1.662294 & 1.026592 & -0.877949 \\ \text { H } & -1.662294 & 1.026592 & 0.877949 \\ \text { C } & 0.384425 & -1.579832 & 1.248957 \\ \text { H } & 0.774971 & -2.067446 & 2.145497 \\ \text { C } & 0.384425 & -1.579832 & -1.248957 \\ \text { H } & 0.774971 & -2.067446 & -2.145497 \\ \text { C } & 0.287700 & 0.634219 & 0.000000 \\ \text { C } & 0.820054 & -0.108962 & 1.244836 \\ \text { H } & 0.452020 & 0.369274 & 2.153277 \\ \text { H } & 1.912044 & -0.040080 & 1.267600 \\ \text { C } & 0.820054 & -0.108962 & -1.244836 \\ \text { H } & 0.452020 & 0.369274 & -2.153277\end{array}$

H $\quad 1.912044-0.040080-1.267600$

C $0.927758-2.282957 \quad 0.000000$

H $\quad 2.020792-2.255961 \quad 0.000000$

H $\quad 0.631821 \quad-3.335446 \quad 0.000000$

H $\quad 0.639295 \quad 1.6659560 .000000$

\section{H from 2tBu:}

Electronic Energy (SCF) = -390.917674020 a.u

Grimme-D3(BJ) Dispersion energy $=-0.0527194770$ a.u

C $\quad 0.009317 \quad 1.442586-1.610970$

H $\quad 0.015660 \quad 2.476989-1.963354$

C $-1.247422 \quad 0.730671-2.125362$

H $\quad-2.142625 \quad 1.255849 \quad-1.781400$

H $\quad-1.267795 \quad 0.741798-3.218408$

C $\quad 1.256491 \quad 0.714964 \quad-2.125362$

H $\quad 1.276313 \quad 0.727043 \quad-3.218408$

H $\quad 2.158910 \quad 1.227643 \quad-1.781400$

C $\quad 0.000000 \quad 1.439581 \quad-0.075756$

H $\quad 0.869099 \quad 1.988478 \quad 0.292074$

$\begin{array}{llll}\text { H } & -0.884568 & 1.975800 & 0.269262\end{array}$

C $-1.253975-0.713224-1.610970$

H $\quad-2.152965-1.224933-1.963354$

C $1.244658-0.729362-1.610970$

H $\quad 2.137305 \quad-1.252056-1.963354$

$\begin{array}{llll}\text { C } & 0.000000 & 0.000000 & 0.489833\end{array}$

$\begin{array}{llll}\text { C } & -1.246714 & -0.719790 & -0.075756\end{array}$

H $\quad-2.156622 \quad-0.241577 \quad 0.292074$

H $\quad-1.268809-1.753959 \quad 0.269262$

C $1.246714-0.719790-0.075756$

H $\quad 2.153377 \quad-0.221842 \quad 0.269262$

H $\quad \begin{array}{llll}1.287523 & -1.746901 & 0.292074\end{array}$

C $\quad-0.009069-1.445635-2.125362$

H $\quad-0.016285-2.483492 \quad-1.781400$

H $\quad-0.008519-1.468841-3.218408$

H $\quad 0.000000 \quad 0.000000 \quad 1.579833$

2H from 2Ph:

Electronic Energy (SCF) $=-390.918287294$ a.u Grimme-D3(BJ) Dispersion energy $=-0.0527012403$ a.u

C $\quad-2.210317 \quad 0.074816 \quad 1.254090$

H $\quad-2.720756 \quad-0.292701 \quad 2.147436$

C $-2.931746-0.4381940 .000000$

H $\quad-2.951487 \quad-1.5313690 .000000$

H $\quad-3.970670 \quad-0.098404 \quad 0.000000$

$\begin{array}{llll}\text { C } & -2.210317 & 1.608435 & 1.252512\end{array}$

H $\quad-3.236096 \quad 1.985795 \quad 1.268718$

H $\quad-1.714863 \quad 1.984140 \quad 2.151650$

C $-0.764195-0.435921 \quad 1.255958$

H $\quad-0.239282 \quad-0.088994 \quad 2.149538$

H $\quad-0.758859 \quad-1.526572 \quad 1.290241$

C $-2.210317 \quad 0.074816-1.254090$

H $\quad-2.720756-0.292701 \quad-2.147436$

C $\quad-1.482829 \quad 2.113209 \quad 0.000000$

H $\quad-1.467196 \quad 3.2055810 .000000$

C $-0.002732 \quad 0.054171 \quad 0.000000$

C $-0.764195-0.435921-1.255958$

H $\quad-0.758859-1.526572-1.290241$

H $\quad-0.239282 \quad-0.088994 \quad-2.149538$

C $\quad-0.038541 \quad 1.594689 \quad 0.000000$ 
$\begin{array}{llll}H & 0.485607 & 1.976870 & 0.879295\end{array}$

H $\quad 0.485607 \quad 1.976870 \quad-0.879295$

C $\quad-2.210317 \quad 1.608435 \quad-1.252512$

H $-1.714863 \quad 1.984140-2.151650$

H $\quad-3.236096 \quad 1.985795-1.268718$

H $\quad 1.011201-0.3458790 .000000$

$2 \mathrm{H}$ from $2 \mathrm{CF}_{3}$ :

Electronic Energy (SCF) = -390.919064819 a.u Grimme-D3(BJ) Dispersion energy $=-0.0527234071 \mathrm{a} . \mathrm{u}$

C $\quad 0.000000 \quad 1.447085-1.553036$

H $\quad 0.000000 \quad 2.479353-1.908788$

C $\quad-1.253462 \quad 0.723686-2.063877$

H $\quad-2.152240 \quad 1.242597 \quad-1.721186$

H $\quad-1.272475 \quad 0.734664-3.156366$

C $\quad 1.253462 \quad 0.723686-2.063877$

H $\quad 1.272475 \quad 0.734664-3.156366$

H $\quad 2.152240 \quad 1.242597 \quad-1.721186$

$\begin{array}{llll}\text { C } & 0.000000 & 1.451490 & -0.018257\end{array}$

H $\quad 0.879393 \quad 1.977975 \quad 0.355706$

H $\quad-0.879393 \quad 1.9779750 .355706$

C $-1.253212-0.723542-1.553036$

H $\quad-2.147183 \quad-1.239677 \quad-1.908788$

C $1.253212-0.723542-1.553036$

$\begin{array}{llll}\text { H } & 2.147183 & -1.239677 & -1.908788\end{array}$

C $0.000000 \quad 0.000000 \quad 0.503109$

$\begin{array}{llll}\text { C } & -1.257027 & -0.725745 & -0.018257\end{array}$

H $\quad-2.152673 \quad-0.227411 \quad 0.355706$

H $\quad-1.273280-1.750565 \quad 0.355706$

$\begin{array}{llll}\text { C } & 1.257027 & -0.725745 & -0.018257\end{array}$

H $\quad 2.152673 \quad-0.2274110 .355706$

H $\quad 1.273280-1.7505650 .355706$

C $0.000000 \quad-1.447373-2.063877$

H $\quad 0.000000-2.485193-1.721186$

H $\quad 0.000000 \quad-1.469328-3.156366$

H $\quad 0.000000 \quad 0.000000 \quad 1.593109$

$\mathbf{2 H}$ from $\mathbf{2} \mathrm{CCl}_{3}$ :

Electronic Energy (SCF) = -390.918752404 a.u

Grimme-D3(BJ) Dispersion energy $=-0.0527366026$ a.u

C $\quad 0.000000 \quad 1.443227 \quad-2.093414$

H $\quad 0.000000 \quad 2.477996-2.442215$

C $\quad 1.252981 \quad 0.723409 \quad-2.605973$

H $\quad 2.151384 \quad 1.242102-2.262116$

H $\quad 1.272238 \quad 0.734527 \quad-3.698477$

C $\quad-1.252981 \quad 0.723409-2.605973$

H $\quad-1.272238 \quad 0.734527 \quad-3.698477$

H $\quad-2.151384 \quad 1.242102-2.262116$

C $\quad 0.000000 \quad 1.448683 \quad-0.558432$

H $\quad-0.877979 \quad 1.978391-0.190446$

H $\quad 0.877979 \quad 1.978391 \quad-0.190446$

C $1.249871 \quad-0.721613-2.093414$

$\begin{array}{llll}\text { H } & 2.146008 & -1.238998 & -2.442215\end{array}$

C $-1.249871-0.721613-2.093414$

H $\quad-2.146008-1.238998-2.442215$

$\begin{array}{llll}\text { C } & 0.000000 & 0.000000 & -0.021004\end{array}$

$\begin{array}{llll}\text { C } & 1.254597 & -0.724342 & -0.558432\end{array}$

H $\quad 2.152326-0.228844 \quad-0.190446$

H $\quad 1.274348-1.749547 \quad-0.190446$
C $\quad-1.254597-0.724342-0.558432$

H $-2.152326-0.228844-0.190446$

H $\quad-1.274348-1.749547 \quad-0.190446$

C $0.000000 \quad-1.446818-2.605973$

H $\quad 0.000000 \quad-2.484204-2.262116$

H $\quad 0.000000 \quad-1.469054-3.698477$

H $\quad 0.000000 \quad 0.000000 \quad 1.068996$

$\mathbf{2 H}$ from $2 \mathrm{CBr}_{3}$ :

Electronic Energy (SCF) = -390.918576430 a.u Grimme-D3(BJ) Dispersion energy $=-0.0527318773 \mathrm{a} . \mathrm{u}$

C $\quad 0.000000 \quad 1.442297 \quad-2.776593$

H $\quad 0.000000 \quad 2.477749 \quad-3.123407$

C $\quad 1.252983 \quad 0.723410 \quad-3.289255$

H $\quad 2.151220 \quad 1.242008-2.944989$

H $\quad \begin{array}{llll}1.272362 & 0.734599 & -4.381736\end{array}$

C $-1.252983 \quad 0.723410 \quad-3.289255$

H $\quad-1.2723620 .734599-4.381736$

$\begin{array}{llll}\mathrm{H} & -2.151220 & 1.242008 & -2.944989\end{array}$

C $\quad 0.000000 \quad 1.448444 \quad-1.241638$

H $\quad-0.877609 \quad 1.979084 \quad-0.874927$

H $\quad 0.877609 \quad 1.979084 \quad-0.874927$

C $1.249066-0.721148-2.776593$

H $\quad 2.145793 \quad-1.238874 \quad-3.123407$

C $\quad-1.249066-0.721148 \quad-2.776593$

H $\quad-2.145793 \quad-1.238874-3.123407$

C $\quad 0.000000 \quad 0.000000 \quad-0.698692$

C $1.254389-0.724222-1.241638$

H $\quad \begin{array}{llll}2.152741 & -0.229510 & -0.874927\end{array}$

H $\quad \begin{array}{llll}1.275132 & -1.749574 & -0.874927\end{array}$

C $\quad-1.254389-0.724222-1.241638$

H $\quad-2.152741-0.229510-0.874927$

H $\quad-1.275132 \quad-1.749574 \quad-0.874927$

$\begin{array}{llll}\text { C } & 0.000000 & -1.446820 & -3.289255\end{array}$

H $\quad 0.000000 \quad-2.484015-2.944989$

H $\quad 0.000000 \quad-1.469197 \quad-4.381736$

$\begin{array}{llll}H & 0.000000 & 0.000000 & 0.391308\end{array}$

$\mathbf{2 H}$ from $2 \mathrm{Cl}_{3}$ :

Electronic Energy (SCF) = -390.918378129 a.u Grimme-D3(BJ) Dispersion energy $=-0.0527316932 \mathrm{a} . \mathrm{u}$

$\begin{array}{llll}\text { C } & 1.248406 & 0.720767 & 3.203168\end{array}$

H $\quad 2.145724 \quad 1.238835 \quad 3.548032$

C $0.000000 \quad 1.446915 \quad 3.715760$

H $\quad 0.000000 \quad 2.483910 \quad 3.371044$

H $\quad 0.000000 \quad 1.469539 \quad 4.808240$

C $\quad 1.253065 \quad-0.723457 \quad 3.715760$

H $1.272658-0.734769 \quad 4.808240$

H $\quad 2.151129 \quad-1.241955 \quad 3.371044$

C $1.252925 \quad 0.723376 \quad 1.668113$

H $2.152749 \quad 0.229912 \quad 1.302881$

H $\quad 1.275484 \quad 1.749379 \quad 1.302881$

$\begin{array}{llll}\text { C } & -1.248406 & 0.720767 & 3.203168\end{array}$

H $\quad-2.145724 \quad 1.238835 \quad 3.548032$

C $0.000000 \quad-1.441535 \quad 3.203168$

$\begin{array}{llll}\text { H } & 0.000000 & -2.477669 & 3.548032\end{array}$

C $\quad 0.000000 \quad 0.000000 \quad 1.118674$

C $\quad-1.252925 \quad 0.723376 \quad 1.668113$

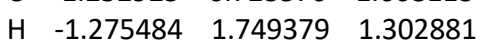


H $\quad-2.152749 \quad 0.229912 \quad 1.302881$

C $0.000000-1.446753 \quad 1.668113$

H $\quad 0.877265 \quad-1.979291 \quad 1.302881$

H $\quad-0.877265-1.979291 \quad 1.302881$

C $-1.253065-0.723457 \quad 3.715760$

H $\quad-2.151129-1.241955 \quad 3.371044$

H $\quad-1.272658 \quad-0.734769 \quad 4.808240$

$\begin{array}{llll}\text { H } & 0.000000 & 0.000000 & 0.028674\end{array}$

\section{$2 \mathrm{H}$ from $2 \mathrm{SiMe}_{3}$ :}

Electronic Energy (SCF) = -390.918606492 a.u

Grimme-D3(BJ) Dispersion energy $=-0.0526607715 \mathrm{a} . \mathrm{u}$

C $\quad-0.000164 \quad 1.447565 \quad-1.879661$

H $\quad-0.000286 \quad 2.479437 \quad-2.239955$

C $-1.253056 \quad 0.723269-2.390145$

H $\quad-2.151833 \quad 1.242015-2.045386$

H $\quad-1.274736 \quad 0.735808-3.483285$

C $\quad 1.252898 \quad 0.723544 \quad-2.390145$

H $1.274596 \quad 0.736050-3.483285$

H $\quad 2.151533 \quad 1.242534 \quad-2.045386$

C $\quad 0.000000 \quad 1.445717 \quad-0.343464$

H $\quad 0.878163 \quad 1.9856930 .020586$

$\begin{array}{llll}H & -0.877997 & 1.985832 & 0.020827\end{array}$

C $-1.253546-0.723925-1.879661$

H $\quad-2.147112-1.239966-2.239955$

C $1.253710-0.723640-1.879661$

H $\quad 2.147398 \quad-1.239471 \quad-2.239955$

$\begin{array}{llll}\text { C } & 0.000000 & 0.000000 & 0.204033\end{array}$

$\begin{array}{llll}\text { C } & -1.252027 & -0.722858 & -0.343464\end{array}$

H $\quad-2.158743 \quad-0.2323350 .020586$

$\begin{array}{llll}\text { H } & -1.280782 & -1.753284 & 0.020827\end{array}$

$\begin{array}{llll}\text { C } & 1.252027 & -0.722858 & -0.343464\end{array}$

H $\quad 2.158780 \quad-0.232548 \quad 0.020827$

H $\quad 1.280579-1.7533590 .020586$

C $0.000159-1.446813-2.390145$

H $\quad 0.000300 \quad-2.484549-2.045386$

H $\quad 0.000140-1.471858 \quad-3.483285$

H $\quad 0.000000 \quad 0.000000 \quad 1.294033$

\section{HCCH from 2CCH:}

Electronic Energy (SCF) $=-77.3675868827$ a.u

Grimme-D3(BJ) Dispersion energy $=-0.0022666708$ a.u
C $\quad 0.000000 \quad 0.000000 \quad 2.490405$
C $\quad 0.000000 \quad 0.000000 \quad 3.691321$
H $\quad 0.000000 \quad 0.000000 \quad 4.752436$
H $\quad 0.000000 \quad 0.000000 \quad 1.410405$

\section{HCN from 2CN:}

Electronic Energy (SCF) = -93.4659323120 a.u

Grimme-D3(BJ) Dispersion energy $=-0.0014547389$ a.u

$$
\begin{array}{cccc}
\mathrm{C} & 0.000000 & 0.000000 & 2.499335 \\
\mathrm{~N} & 0.000000 & 0.000000 & 3.650132 \\
\mathrm{H} & 0.000000 & 0.000000 & 1.419335
\end{array}
$$

$\mathrm{CH}_{4}$ from 2Me:

Electronic Energy (SCF) $=-40.5407433337$ a.u
Grimme-D3(BJ) Dispersion energy $=-0.0019133217 \mathrm{a} . \mathrm{u}$

C $\quad 0.000000 \quad 0.000000 \quad 2.771933$

H $\quad 0.8829450 .509769 \quad 3.163603$

H $\quad-0.882945 \quad 0.5097693 .163603$

H $\quad 0.000000 \quad-1.019537 \quad 3.163603$

H $\quad 0.000000 \quad 0.000000 \quad 1.681933$

\section{Ethane from 2Et:}

Electronic Energy (SCF) $=-79.8707220460$ a.u Grimme-D3(BJ) Dispersion energy $=-0.0054114696$ a.u

$\begin{array}{lrrr}\text { C } & 2.376443 & 0.698371 & 0.000000 \\ \text { H } & 2.561065 & 1.330773 & -0.874145 \\ \text { H } & 2.561065 & 1.330774 & 0.874145 \\ \text { C } & 3.381152 & -0.452365 & 0.000000 \\ \text { H } & 4.401031 & -0.065724 & 0.000000 \\ \text { H } & 3.273560 & -1.085829 & 0.880721 \\ \text { H } & 3.273559 & -1.085829 & -0.880721 \\ \text { H } & 1.321159 & 0.425468 & 0.000000\end{array}$

Propane from 2iPr:

Electronic Energy (SCF) $=-119.203279105$ a.u Grimme-D3(BJ) Dispersion energy $=-0.0099946732 \mathrm{a} . \mathrm{u}$

C $\quad 0.789127 \quad 2.105628 \quad 0.000000$

H $\quad 1.883585 \quad 2.039457 \quad 0.000000$

C $0.384425 \quad 2.901496-1.246103$

H $\quad 0.813970 \quad 3.903333-1.202893$

H $\quad 0.728707 \quad 2.439676 \quad-2.169422$

H $\quad-0.698753 \quad 3.017109-1.310625$

C $\quad 0.384425 \quad 2.901496 \quad 1.246103$

H $\quad 0.728707 \quad 2.439676 \quad 2.169422$

H $\quad 0.813970 \quad 3.903333 \quad 1.202893$

H $\quad-0.698753 \quad 3.017109 \quad 1.310625$

H $\quad 0.437532 \quad 1.073891 \quad 0.000000$

Iso-Butane from 2tBu:

Electronic Energy (SCF) = -158.537425974 a.u Grimme-D3(BJ) Dispersion energy $=-0.0156888044$ a.u

$\begin{array}{llll}C & 0.000000 & 0.000000 & 2.068802\end{array}$

C $1.336654 \quad 0.514828 \quad 2.631143$

H $\quad 1.2591290 .624756 \quad 3.713995$

H $\quad 2.155802 \quad-0.174874 \quad 2.432851$

H $\quad 1.607033 \quad 1.488446 \quad 2.223378$

C $\quad-1.114181 \quad 0.900163 \quad 2.631143$

$\begin{array}{llll}\text { H } & -2.092548 & 0.647509 & 2.223378\end{array}$

H $\quad-1.170619 \quad 0.778060 \quad 3.713995$

H $\quad-0.926455 \quad 1.954416 \quad 2.432851$

C $-0.222473-1.414990 \quad 2.631143$

H $\quad-0.088510-1.402816 \quad 3.713995$

H $\quad-1.229346-1.779542 \quad 2.432851$

H $\quad 0.485515 \quad-2.135954 \quad 2.223378$

H $\quad 0.000000 \quad 0.000000 \quad 0.978802$

Ph from 2Ph:

Electronic Energy (SCF) $=-232.358243046$ a.u

Grimme-D3(BJ) Dispersion energy $=-0.0189486433 \mathrm{a} . \mathrm{u}$ 


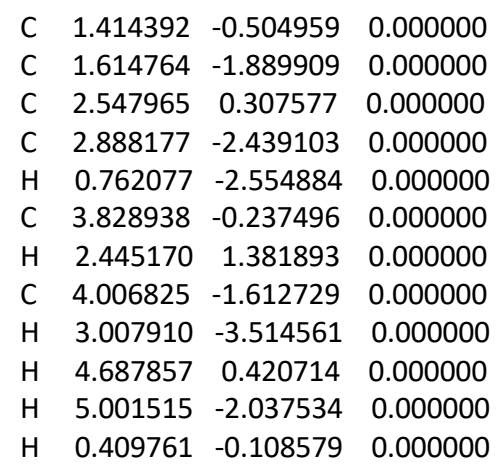

$\mathrm{HCF}_{3}$ from $2 \mathrm{CF}_{3}$ :

Electronic Energy (SCF) $=-338.395438548$ a.u Grimme-D3(BJ) Dispersion energy $=-0.0026761791 \mathrm{a} . \mathrm{u}$

$\begin{array}{rrrr}\text { C } & 0.000000 & 0.000000 & 2.023825 \\ \text { F } & -1.083712 & 0.625681 & 2.536898 \\ \text { F } & 0.000000 & -1.251363 & 2.536898 \\ \text { F } & 1.083712 & 0.625681 & 2.536898 \\ \text { H } & 0.000000 & 0.000000 & 0.933825\end{array}$

$\mathrm{HCCl}_{3}$ from $2 \mathrm{CCl}_{3}$ :

Electronic Energy (SCF) = -1419.39901620 a.u Grimme-D3(BJ) Dispersion energy $=-0.0102818983 \mathrm{a} . \mathrm{u}$

C $\quad 0.000000 \quad 0.000000 \quad 1.528161$

Cl $1.448920 \quad 0.836534 \quad 2.195076$

Cl $\quad-1.448920 \quad 0.836534 \quad 2.195076$

Cl $\quad 0.000000 \quad-1.673069 \quad 2.195076$

H $\quad 0.000000 \quad 0.000000 \quad 0.438161$

$\mathrm{HCBr}_{3}$ from $2 \mathrm{CBr}_{3}$ :

Electronic Energy (SCF) = -7761.26307097 a.u

Grimme-D3(BJ) Dispersion energy $=-0.0129826748$ a.u

$\begin{array}{lccc}\mathrm{C} & 0.000000 & 0.000000 & 0.847843 \\ \mathrm{Br} & 0.000000 & -1.833142 & 1.592760 \\ \mathrm{Br} & -1.587548 & 0.916571 & 1.592760 \\ \mathrm{Br} & 1.587548 & 0.916571 & 1.592760 \\ \mathrm{H} & 0.000000 & 0.000000 & -0.242157\end{array}$

$\mathrm{HCl}_{3}$ from $2 \mathrm{Cl}_{3}$ :

Electronic Energy (SCF) $=-932.121101548$ a.u

Grimme-D3(BJ) Dispersion energy $=-0.0176791069$ a.u

C $\quad 0.000000 \quad 0.000000 \quad-0.429289$

I $0.000000 \quad 2.036034-1.268568$

| $1.763258-1.018017-1.268568$

l $\quad-1.763258-1.018017-1.268568$

H $\quad 0.000000 \quad 0.000000 \quad 0.660711$

$\mathrm{HSiMe}_{3}$ from $2 \mathrm{SiMe}_{3}$ :

Electronic Energy (SCF) $=-409.969084054$ a.u Grimme-D3(BJ) Dispersion energy $=-0.0176498629$ a.u
$\begin{array}{llll}C & 1.526997 & 0.889504 & 2.750345\end{array}$

H $\quad 1.533737 \quad 0.890333 \quad 3.842182$

H $\quad 2.446552 \quad 0.406390 \quad 2.416477$

H $\quad 1.557712 \quad 1.9291632 .420802$

C $\quad-1.533832 \quad 0.877666 \quad 2.750345$

$\begin{array}{llll}H & -2.449560 & 0.384437 & 2.420802\end{array}$

H $\quad-1.5379190 .883089 \quad 3.842182$

H $\quad-1.575220 \quad 1.915581 \quad 2.416477$

$\begin{array}{llll}\text { C } & 0.006835 & -1.767170 & 2.750345\end{array}$

H $\quad 0.004183-1.773421 \quad 3.842182$

H $\quad-0.871332 \quad-2.321971 \quad 2.416477$

H $\quad 0.891848 \quad-2.313599 \quad 2.420802$

Si $\quad 0.000000 \quad 0.000000 \quad 2.103694$

H $\quad 0.000000 \quad 0.000000 \quad 0.623694$

\section{H from 3F:}

Electronic Energy (SCF) = -508.928319726 a.u

Grimme-D3(BJ) Dispersion energy $=-0.0696963248 \mathrm{a} . \mathrm{u}$

$\begin{array}{llll}C & 1.258580 & 2.193619 & 0.084762\end{array}$

$\begin{array}{llll}\text { H } & 2.152601 & 2.695041 & -0.292813\end{array}$

H $\quad 1.318738 \quad 2.221163 \quad 1.175583$

$\begin{array}{llll}\text { C } & 0.000000 & 2.925657 & -0.376149\end{array}$

H $\quad 0.000000 \quad 2.993819-1.469266$

H $\quad 0.000000 \quad 3.950839-0.000516$

C $1.271125 \quad 0.733884-0.372397$

H $\quad \begin{array}{llll}\text { H.250235 } & 0.721823 & -1.468974\end{array}$

C $\quad-1.258580 \quad 2.193619 \quad 0.084762$

H $\quad-1.318738 \quad 2.221163 \quad 1.175583$

H $\quad-2.152601 \quad 2.695041 \quad-0.292813$

$\begin{array}{llll}\text { C } & 0.000000 & 0.000000 & 0.072252\end{array}$

$\begin{array}{llll}\text { C } & -1.271125 & 0.733884 & -0.372397\end{array}$

H $\quad-1.250235 \quad 0.721823-1.468974$

$\begin{array}{llll}\text { C } & 2.529020 & -0.006847 & 0.084762\end{array}$

H $\quad 3.410274 \quad 0.516687 \quad-0.292813$

H $\quad 2.582952 \quad 0.031479 \quad 1.175583$

$\begin{array}{llll}\text { C } & -2.529020 & -0.006847 & 0.084762\end{array}$

$\begin{array}{llll}\text { H } & -3.410274 & 0.516687 & -0.292813\end{array}$

H $\quad-2.582952 \quad 0.031479 \quad 1.175583$

C $0.000000 \quad-1.467768-0.372397$

$\begin{array}{llll}\text { H } & 0.000000 & -1.443647 & -1.468974\end{array}$

$\begin{array}{llll}\text { C } & 1.270440 & -2.186772 & 0.084762\end{array}$

H $\quad 1.264215 \quad-2.252642 \quad 1.175583$

H $\quad 1.257673 \quad-3.211728 \quad-0.292813$

C $\quad-1.270440-2.1867720 .084762$

H $\quad-1.264215 \quad-2.252642 \quad 1.175583$

H $-1.257673-3.211728-0.292813$

C $-2.533693 \quad-1.462828-0.376149$

H $\quad-2.592723 \quad-1.496910-1.469266$

H $\quad-3.421527-1.975420-0.000516$

C $2.533693-1.462828-0.376149$

H $2.592723 \quad-1.496910-1.469266$

H $3.421527-1.975420-0.000516$

H $\quad 0.000000 \quad 0.000000 \quad 1.162252$

3H from 3Cl:

Electronic Energy (SCF) $=-508.926857504$ a.u

Grimme-D3(BJ) Dispersion energy $=-0.0694860270$ a.u

C $\quad 1.266896 \quad 2.205460 \quad-0.104214$

H $\quad 2.150479 \quad 2.687879-0.528082$ 
$\begin{array}{llll}\text { H } & 1.357321 & 2.280143 & 0.981146\end{array}$

C $0.000000 \quad 2.922634-0.572477$

$\begin{array}{llll}\text { H } & 0.000000 & 2.969749 & -1.666576\end{array}$

H $\quad 0.000000 \quad 3.955199 \quad-0.218107$

$\begin{array}{llll}\text { C } & 1.266120 & 0.730994 & -0.505929\end{array}$

H $\quad 1.198450 \quad 0.691925 \quad-1.601597$

$\begin{array}{llll}\text { C } & -1.266896 & 2.205460 & -0.104214\end{array}$

$\begin{array}{llll}\text { H } & -1.357321 & 2.280143 & 0.981146\end{array}$

$\begin{array}{llll}\text { H } & -2.150479 & 2.687879 & -0.528082\end{array}$

C $\quad 0.000000 \quad 0.000000-0.032125$

$\begin{array}{llll}\text { C } & -1.266120 & 0.730994 & -0.505929\end{array}$

$\begin{array}{llll}\text { H } & -1.198450 & 0.691925 & -1.601597\end{array}$

$\begin{array}{llll}\text { C } & 2.543433 & -0.005566 & -0.104214\end{array}$

H $3.4030110 .518430-0.528082$

$\begin{array}{llll}\text { H } & 2.653322 & 0.035403 & 0.981146\end{array}$

$\begin{array}{llll}\text { C } & -2.543433 & -0.005566 & -0.104214\end{array}$

$\begin{array}{llll}\text { H } & -3.403011 & 0.518430 & -0.528082\end{array}$

$\begin{array}{llll}H & -2.653322 & 0.035403 & 0.981146\end{array}$

$\begin{array}{llll}\text { C } & 0.000000 & -1.461989 & -0.505929\end{array}$

H $\quad 0.000000 \quad-1.383850 \quad-1.601597$

$\begin{array}{llll}\text { C } & 1.276537 & -2.199894 & -0.104214\end{array}$

$\begin{array}{llll}\text { H } & 1.296001 & -2.315546 & 0.981146\end{array}$

H $\quad 1.252532 \quad-3.206309-0.528082$

$\begin{array}{llll}\text { C } & -1.276537 & -2.199894 & -0.104214\end{array}$

$\begin{array}{llll}\text { H } & -1.296001 & -2.315546 & 0.981146\end{array}$

$\begin{array}{llll}\text { H } & -1.252532 & -3.206309 & -0.528082\end{array}$

$\begin{array}{llll}\text { C } & -2.531076 & -1.461317 & -0.572477\end{array}$

H $\quad-2.571878-1.484874-1.666576$

$\begin{array}{llll}H & -3.425303 & -1.977600 & -0.218107\end{array}$

$\begin{array}{llll}\text { C } & 2.531076 & -1.461317 & -0.572477\end{array}$

H $\quad 2.571878-1.484874-1.666576$

H $3.425303 \quad-1.977600 \quad-0.218107$

H $\quad 0.000000 \quad 0.000000 \quad 1.057875$

3H from 3Br:

Electronic Energy (SCF) = -508.925971928 a.u Grimme-D3(BJ) Dispersion energy $=-0.0694295745$ a.u

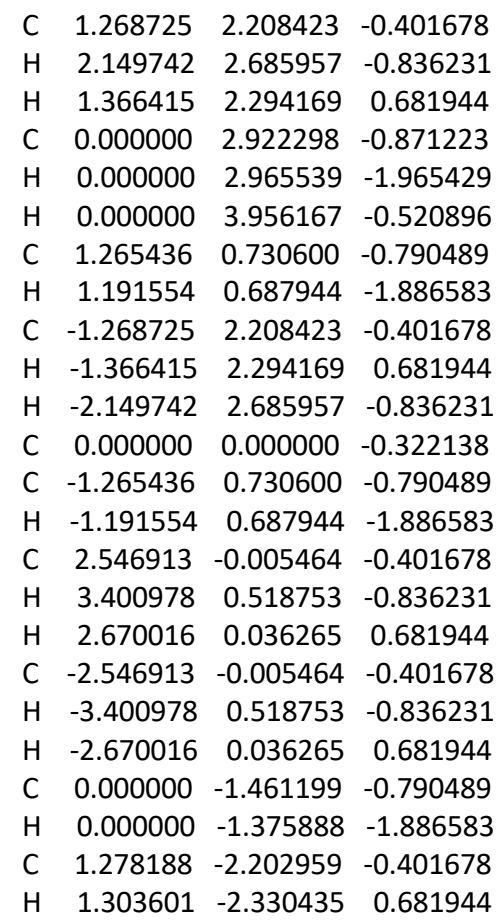

$\begin{array}{llll}\text { H } & 1.251236 & -3.204710 & -0.836231\end{array}$

C $-1.278188-2.202959-0.401678$

H $-1.303601-2.3304350 .681944$

H $\quad-1.251236-3.204710 \quad-0.836231$

C $-2.530785-1.461149-0.871223$

H $\quad-2.568232-1.482770-1.965429$

H $\quad-3.426141-1.978084-0.520896$

C $2.530785-1.461149-0.871223$

H $\quad 2.568232-1.482770-1.965429$

H $3.426141-1.978084-0.520896$

H $\quad 0.000000 \quad 0.000000 \quad 0.767862$

3H from 3I:

Electronic Energy (SCF) $=-508.925107806$ a.u

Grimme-D3(BJ) Dispersion energy $=-0.0693681957 \mathrm{a} . \mathrm{u}$

C $\quad-1.279565 \quad 2.206069-0.692382$

H $\quad-1.249398 \quad 3.202873 \quad-1.138023$

H $\quad-1.312491 \quad 2.347310 \quad 0.389420$

$\begin{array}{llll}\text { C } & -2.530289 & 1.460863 & -1.163039\end{array}$

H $\quad-2.564052 \quad 1.480356-2.257274$

H $\quad-3.426872 \quad 1.978506 \quad-0.817073$

C $0.000000 \quad 1.459328-1.065965$

H $\quad 0.000000 \quad 1.364461-2.162164$

C $\quad-2.550294 \quad 0.005101-0.692382$

H $-2.689076-0.037005 \quad 0.389420$

H $\quad-3.398468-0.519426-1.138023$

$\begin{array}{llll}\text { C } & 0.000000 & 0.000000 & -0.593797\end{array}$

$\begin{array}{llll}\text { C } & -1.263815 & -0.729664 & -1.065965\end{array}$

H $\quad-1.181658 \quad-0.682231 \quad-2.162164$

C $1.279565 \quad 2.206069-0.692382$

H $\quad 1.249398 \quad 3.202873 \quad-1.138023$

H $\quad \begin{array}{llll}\text { H } & 1.312491 & 2.347310 & 0.389420\end{array}$

C $-1.270730-2.211170-0.692382$

$\begin{array}{llll}\text { H } & -2.149070 & -2.683447 & -1.138023\end{array}$

H $\quad-1.376585-2.310306 \quad 0.389420$

C $1.263815-0.729664-1.065965$

H $\quad 1.181658-0.682231-2.162164$

C $2.550294 \quad 0.005101 \quad-0.692382$

H $2.689076-0.037005 \quad 0.389420$

H $3.398468 \quad-0.519426 \quad-1.138023$

C $1.270730-2.211170-0.692382$

H $\quad 1.376585-2.310306 \quad 0.389420$

H $\quad 2.149070 \quad-2.683447 \quad-1.138023$

C $0.000000-2.921726-1.163039$

H $\quad 0.000000 \quad-2.960712-2.257274$

H $\quad 0.000000 \quad-3.957011 \quad-0.817073$

C $2.530289 \quad 1.460863 \quad-1.163039$

H $2.564052 \quad 1.480356 \quad-2.257274$

H $3.426872 \quad 1.978506 \quad-0.817073$

H $\quad 0.000000 \quad 0.000000 \quad 0.496203$

\section{H from 3CCH:}

Electronic Energy (SCF) = -508.926713667 a.u Grimme-D3(BJ) Dispersion energy $=-0.0694715221$ a.u
C $\quad \begin{array}{lll}1.267049 & 2.203183 & -0.084831\end{array}$
H $2.151496 \quad 2.692879-0.499329$
H $\quad 1.351517 \quad 2.270212 \quad 1.002963$
$\begin{array}{llll}\text { C } & 0.000000 & 2.922442 & -0.550605\end{array}$
H $\quad 0.000000 \quad 2.972371-1.644480$ 
$\begin{array}{llll}\text { H } & 0.000000 & 3.954188 & -0.193211\end{array}$

C $1.267148 \quad 0.731588-0.493854$

H $\quad 1.191113 \quad 0.687689-1.587220$

C $\quad-1.267049 \quad 2.203183-0.084831$

H $\quad-1.351517 \quad 2.270212 \quad 1.002963$

H $\quad-2.151496 \quad 2.692879 \quad-0.499329$

C $0.000000 \quad 0.000000 \quad 0.031762$

$\begin{array}{llll}\text { C } & -1.267148 & 0.731588 & -0.493854\end{array}$

H $\quad-1.1911130 .687689 \quad-1.587220$

$\begin{array}{llll}\text { C } & 2.541537 & -0.004295 & -0.084831\end{array}$

$\begin{array}{llll}\text { H } & 3.407850 & 0.516810 & -0.499329\end{array}$

$\begin{array}{llll}\text { H } & 2.641820 & 0.035342 & 1.002963\end{array}$

$\begin{array}{llll}\text { C } & -2.541537 & -0.004295 & -0.084831\end{array}$

H $\quad-3.407850 \quad 0.516810 \quad-0.499329$

$\begin{array}{llll}\text { H } & -2.641820 & 0.035342 & 1.002963\end{array}$

C $0.000000 \quad-1.463177 \quad-0.493854$

H $\quad 0.000000 \quad-1.375379-1.587220$

C $1.274488-2.198888-0.084831$

H $\quad 1.290303 \quad-2.305554 \quad 1.002963$

H $\quad 1.256354-3.209690-0.499329$

C $\quad-1.274488-2.198888-0.084831$

H $\quad-1.290303 \quad-2.305554 \quad 1.002963$

H $-1.256354-3.209690-0.499329$

$\begin{array}{llll}\text { C } & -2.530909 & -1.461221 & -0.550605\end{array}$

H $\quad-2.574149-1.486186-1.644480$

H $\quad-3.424427-1.977094-0.193211$

C $2.530909-1.461221-0.550605$

H $2.574149-1.486186-1.644480$

H $3.424427-1.977094 \quad-0.193211$

H $\quad 0.000000 \quad 0.000000 \quad 1.121762$

\section{H from $3 \mathrm{CN}$ :}

Electronic Energy (SCF) $=-508.927356608$ a.u Grimme-D3(BJ) Dispersion energy $=-0.0694334525$ a.u

$\begin{array}{llll}\text { C } & 1.266181 & 2.203024 & -0.065202\end{array}$

H $\quad 2.151877 \quad 2.696252-0.471484$

H $\quad 1.348306 \quad 2.262558 \quad 1.023673$

C $\quad 0.000000 \quad 2.924723 \quad-0.528438$

H $\quad 0.000000 \quad 2.981673-1.621780$

H $\quad 0.000000 \quad 3.953395 \quad-0.163754$

C $\quad 1.272580 \quad 0.734724-0.487530$

H $\quad 1.2060620 .696320-1.581076$

$\begin{array}{llll}\text { C } & -1.266181 & 2.203024 & -0.065202\end{array}$

H $\quad-1.348306 \quad 2.262558 \quad 1.023673$

H $\quad-2.151877 \quad 2.696252 \quad-0.471484$

$\begin{array}{llll}\text { C } & 0.000000 & 0.000000 & 0.014732\end{array}$

C $-1.272580 \quad 0.734724-0.487530$

H $\quad-1.2060620 .696320-1.581076$

C $2.540966-0.004967-0.065202$

H $3.4109610 .515454 \quad-0.471484$

H $\quad 2.633586 \quad 0.036388 \quad 1.023673$

$\begin{array}{llll}\text { C } & -2.540966 & -0.004967 & -0.065202\end{array}$

H $\quad-3.410961 \quad 0.515454-0.471484$

H $\quad-2.633586 \quad 0.036388 \quad 1.023673$

C $0.000000-1.469449-0.487530$

H $\quad 0.000000-1.392641-1.581076$

$\begin{array}{llll}\text { C } & 1.274785 & -2.198057 & -0.065202\end{array}$

H $\quad 1.285280-2.298946 \quad 1.023673$

H $\quad 1.259084 \quad-3.211706-0.471484$

C $-1.274785-2.198057-0.065202$

H $\quad-1.285280-2.298946 \quad 1.023673$
H $\quad-1.259084 \quad-3.211706-0.471484$

C $-2.532884-1.462361-0.528438$

H $\quad-2.582205-1.490837-1.621780$

H $\quad-3.423741-1.976698-0.163754$

C $2.532884-1.462361-0.528438$

H $\quad 2.582205-1.490837 \quad-1.621780$

H $3.423741 \quad-1.976698 \quad-0.163754$

H $\quad 0.000000 \quad 0.000000 \quad 1.104732$

\section{H from 3Me:}

Electronic Energy (SCF) = -508.924513821 a.u

Grimme-D3(BJ) Dispersion energy $=-0.0693195793 \mathrm{a} . \mathrm{u}$

$\begin{array}{llll}\text { C } & 1.271953 & 2.208022 & 0.026813\end{array}$

H $\quad 2.147748 \quad 2.681533 \quad-0.424148$

H $\quad 1.389913 \quad 2.322040 \quad 1.107558$

C $0.000000 \quad 2.920422-0.440030$

H $\quad 0.000000 \quad 2.953438-1.534363$

H $\quad 0.000000 \quad 3.958139-0.099577$

C $1.2539340 .723959-0.342629$

H $\quad \begin{array}{llll}\text { H } & 1.164968 & 0.672595 & -1.436074\end{array}$

C $\quad-1.271953 \quad 2.208022 \quad 0.026813$

H $\quad-1.389913 \quad 2.322040 \quad 1.107558$

H $\quad-2.147748 \quad 2.681533 \quad-0.424148$

C $\quad 0.000000 \quad 0.000000 \quad 0.211457$

$\begin{array}{llll}\text { C } & -1.253934 & 0.723959 & -0.342629\end{array}$

H $\quad-1.164968 \quad 0.672595 \quad-1.436074$

$\begin{array}{llll}\text { C } & 2.548180 & -0.002467 & 0.026813\end{array}$

H $\quad 3.396150 \quad 0.519237 \quad-0.424148$

H $\quad \begin{array}{llll}2.705902 & 0.042680 & 1.107558\end{array}$

$\begin{array}{llll}\text { C } & -2.548180 & -0.002467 & 0.026813\end{array}$

H $\quad-3.396150 \quad 0.519237 \quad-0.424148$

H $\quad-2.705902 \quad 0.042680 \quad 1.107558$

C $0.000000 \quad-1.447918-0.342629$

H $\quad 0.000000 \quad-1.345189-1.436074$

C $\quad 1.276227-2.205555 \quad 0.026813$

H $\quad 1.315989-2.364720 \quad 1.107558$

H $\quad 1.248402-3.200771-0.424148$

$\begin{array}{llll}\text { C } & -1.276227 & -2.205555 & 0.026813\end{array}$

H $\quad-1.315989-2.364720 \quad 1.107558$

H $-1.248402-3.200771-0.424148$

C $-2.529159-1.460211-0.440030$

H $\quad-2.557752 \quad-1.476719-1.534363$

H $\quad-3.427849-1.979070-0.099577$

C $2.529159-1.460211-0.440030$

H $\quad 2.557752 \quad-1.476719-1.534363$

H $\quad 3.427849-1.979070-0.099577$

H $\quad 0.000000 \quad 0.000000 \quad 1.301457$

\section{H from 3Et:}

Electronic Energy (SCF) = -508.919883007 a.u Grimme-D3(BJ) Dispersion energy $=-0.0692389277$ a.u
C $\quad 0.889744 \quad 2.342489 \quad-0.208002$
H $\quad 1.676955 \quad 2.925451 \quad-0.692931$
H $\quad 1.0426892 .455762 \quad 0.867977$
$\begin{array}{llll}\text { C } & -0.481929 & 2.895479 & -0.604821\end{array}$
H $\quad-0.538298 \quad 2.923895-1.697716$
H $\quad-0.592775 \quad 3.926604-0.262414$
C $1.0156320 .873009-0.612313$
H $\quad 0.764844 \quad 0.832580 \quad-1.681454$ 


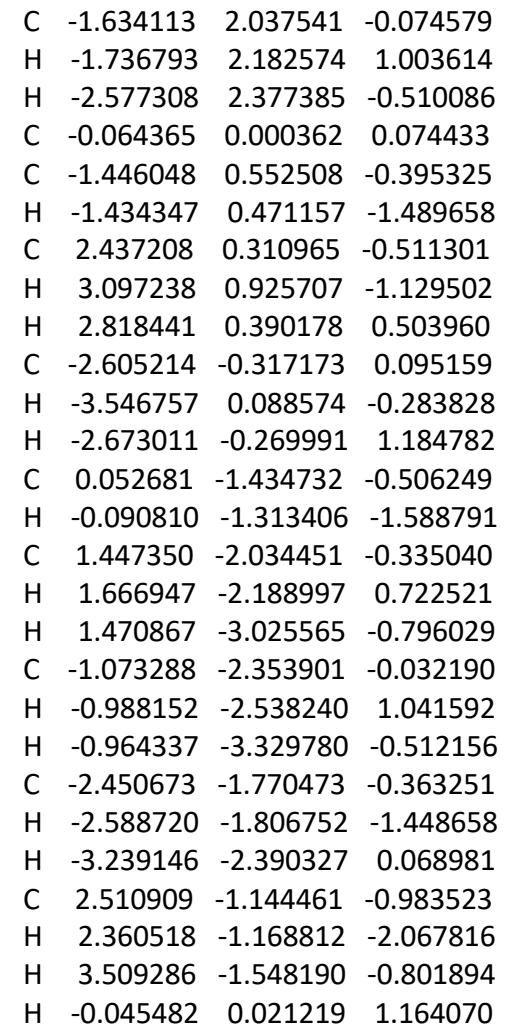

3H from 3iPr:

Electronic Energy (SCF) $=-508.911815262$ a.u Grimme-D3(BJ) Dispersion energy $=-0.0690961247 \mathrm{a} . \mathrm{u}$

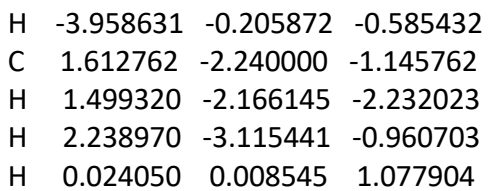

3H from 3tBu:

Electronic Energy (SCF) = -508.901051129 a.u

Grimme-D3(BJ) Dispersion energy $=-0.0689477207$ a.u

C $\quad-0.006853 \quad 2.582026-0.628990$

H $\quad 0.500059 \quad 3.279680 \quad-1.301651$

H $\quad 0.084867 \quad 3.0144390 .361223$

C $-1.484600 \quad 2.493271-1.036956$

H $\quad-1.538600 \quad 2.415595-2.127381$

H $\quad-2.000920 \quad 3.417862 \quad-0.769852$

C $\quad 0.713390 \quad 1.230798 \quad-0.739598$

H $\quad 0.654193 \quad 0.998770 \quad-1.810221$

C $\quad-2.214474 \quad 1.278527 \quad-0.451491$

H $\quad-2.390010 \quad 1.4063240 .616066$

H $\quad-3.202926 \quad 1.199819-0.912061$

C $0.000000 \quad 0.000000 \quad-0.079999$

C $\quad-1.422597 \quad 0.002415-0.739598$

H $\quad-1.192057 \quad 0.067163 \quad-1.810221$

C $\quad 2.214474 \quad 1.278527 \quad-0.451491$

H $\quad 2.640537 \quad 2.173906 \quad-0.912061$

H $\quad 2.412917 \quad 1.366647 \quad 0.616066$

C $-2.232674-1.296948-0.628990$

H $\quad-3.090316-1.206776-1.301651$

H $\quad-2.653014 \quad-1.433723 \quad 0.361223$

C $\quad 0.709207-1.233212-0.739598$

H $\quad 0.537863 \quad-1.065933 \quad-1.810221$

C $2.239527-1.285078-0.628990$

H $\quad 2.568148 \quad-1.580717 \quad 0.361223$

H $\quad 2.590257-2.072904-1.301651$

C $\quad 0.000000-2.557055-0.451491$

H $\quad-0.022907-2.772971 \quad 0.616066$

H $\quad 0.562389-3.373725 \quad-0.912061$

C $-1.416936-2.532337-1.036956$

H $\quad-1.322666-2.540264 \quad-2.127381$

H $\quad-1.959496-3.441779-0.769852$

C $2.9015360 .039066-1.036956$

H $\quad 2.861266 \quad 0.124670-2.127381$

H $3.960416 \quad 0.023917 \quad-0.769852$

H $\quad 0.000000 \quad 0.000000 \quad 1.010001$

3H from 3Ph:

Electronic Energy (SCF) = -508.913384647 a.u Grimme-D3(BJ) Dispersion energy $=-0.0690952501 \mathrm{a} . \mathrm{u}$
$\begin{array}{llll}\text { C } & 2.233219 & -0.878047 & 1.292528\end{array}$
H $\quad 2.644234 \quad-1.4428542 .133459$
H $\quad 2.456991 \quad 0.165408 \quad 1.507901$
C $2.915059-1.351841 \quad 0.000000$
$\begin{array}{llll}\text { H } & 2.900817 & -2.446272 & 0.000000\end{array}$
H $3.967955-1.0618820 .000000$
C $0.724801-1.115506 \quad 1.253650$
H $\quad 0.610766-2.201800 \quad 1.144971$
C $2.233219 \quad-0.878047-1.292528$
H $\quad 2.456991 \quad 0.165408 \quad-1.507901$
H $\quad 2.644234-1.442854-2.133459$ 
C $0.001774 \quad-0.532926 \quad 0.000000$

C $0.724801-1.115506-1.253650$

H $\quad 0.610766-2.201800 \quad-1.144971$

C $\quad-0.008485-0.733398 \quad 2.536901$

H $\quad 0.552971 \quad-1.0898193 .404086$

$\begin{array}{llll}\text { H } & -0.063268 & 0.354915 & 2.623461\end{array}$

C $-0.008485-0.733398-2.536901$

H $\quad 0.552971-1.089819-3.404086$

H $\quad-0.063268 \quad 0.354915 \quad-2.623461$

C $-1.414191-1.1986360 .000000$

H $\quad-1.183499-2.270403 \quad 0.000000$

C $-2.215096-0.973755 \quad 1.291565$

$\begin{array}{llll}\text { H } & -2.550958 & 0.057068 & 1.382379\end{array}$

H $\quad-3.121550-1.583378 \quad 1.245659$

C $-2.215096-0.973755-1.291565$

H $\quad-2.550958 \quad 0.057068-1.382379$

H $\quad-3.121550-1.583378-1.245659$

C $-1.412525 \quad-1.342890-2.543736$

H $\quad-1.313526-2.431882-2.596425$

H $-1.960086-1.037878-3.437869$

C $-1.412525 \quad-1.342890 \quad 2.543736$

$\begin{array}{llll}\text { H } & -1.313526 & -2.431882 & 2.596425\end{array}$

H $\quad-1.960086-1.037878 \quad 3.437869$

H $\quad-0.013267 \quad 0.556970 \quad 0.000000$

3H from $3 \mathrm{CF}_{3}$ :

Electronic Energy (SCF) $=-508.917349115$ a.u Grimme-D3(BJ) Dispersion energy $=-0.0689428050 \mathrm{a} . \mathrm{u}$

C $\quad \begin{array}{lll}1.283765 & 2.225682 & -0.430158\end{array}$

H $\quad 2.139314 \quad 2.659253 \quad-0.953641$

H $\quad 1.449523 \quad 2.408341 \quad 0.630374$

C $\quad 0.000000 \quad 2.916879-0.905531$

H $\quad 0.000000 \quad 2.921087 \quad-2.000483$

$\begin{array}{llll}H & 0.000000 & 3.963117 & -0.594407\end{array}$

C $\quad 1.253512 \quad 0.723716-0.723455$

H $\quad 1.102010 \quad 0.636246 \quad-1.805999$

C $\quad-1.283765 \quad 2.225682-0.430158$

$\begin{array}{llll}\text { H } & -1.449523 & 2.408341 & 0.630374\end{array}$

H $\quad-2.139314 \quad 2.659253 \quad-0.953641$

C $\quad 0.000000 \quad 0.000000 \quad-0.144345$

$\begin{array}{llll}\text { C } & -1.253512 & 0.723716 & -0.723455\end{array}$

H $\quad-1.102010 \quad 0.636246 \quad-1.805999$

C $2.569379-0.001068-0.430158$

$\begin{array}{llll}\text { H } & 3.372638 & 0.523073 & -0.953641\end{array}$

H $\quad 2.810446 \quad 0.051153 \quad 0.630374$

$\begin{array}{llll}\text { C } & -2.569379 & -0.001068 & -0.430158\end{array}$

$\begin{array}{llll}\text { H } & -3.372638 & 0.523073 & -0.953641\end{array}$

$\begin{array}{llll}\text { H } & -2.810446 & 0.051153 & 0.630374\end{array}$

$\begin{array}{llll}\text { C } & 0.000000 & -1.447431 & -0.723455\end{array}$

H $\quad 0.000000 \quad-1.272491-1.805999$

C $1.285614 \quad-2.224614-0.430158$

$\begin{array}{llll}\text { H } & 1.360923 & -2.459494 & 0.630374\end{array}$

H $\quad 1.233324-3.182327 \quad-0.953641$

C $-1.285614-2.224614 \quad-0.430158$

$\begin{array}{llll}\text { H } & -1.360923 & -2.459494 & 0.630374\end{array}$

H $-1.233324-3.182327 \quad-0.953641$

$\begin{array}{llll}\text { C } & -2.526091 & -1.458440 & -0.905531\end{array}$

H $\quad-2.529735-1.460543 \quad-2.000483$

H $\quad-3.432160-1.981559-0.594407$

C $2.526091-1.458440-0.905531$

H $\quad 2.529735 \quad-1.460543 \quad-2.000483$
H $\quad 3.432160 \quad-1.981559-0.594407$

H $\quad 0.000000 \quad 0.0000000 .945655$

3H from $\mathbf{3} \mathbf{C C l}_{3}$ :

Electronic Energy (SCF) = -508.900307310 a.u

Grimme-D3(BJ) Dispersion energy $=-0.0687141768 \mathrm{a} . \mathrm{u}$

C $\quad 0.000000 \quad 2.587275 \quad-0.970380$

H $\quad 0.497874 \quad 3.275488$ - 1.658588

$\begin{array}{llll}H & 0.124753 & 3.014617 & 0.017965\end{array}$

C $\quad-1.486350 \quad 2.505339-1.356606$

H $\quad-1.549872 \quad 2.466650-2.448664$

H $\quad-1.997103 \quad 3.421359-1.054352$

C $0.719346 \quad 1.239814 \quad-1.090240$

H $\quad 0.644063 \quad 0.991884-2.154558$

C $\quad-2.230041 \quad 1.276193-0.813693$

H $\quad-2.455769 \quad 1.378171 \quad 0.245047$

H $\quad-3.192832 \quad 1.195707 \quad-1.324244$

C $0.000000 \quad 0.000000 \quad-0.445202$

$\begin{array}{llll}\text { C } & -1.433383 & 0.003065 & -1.090240\end{array}$

$\begin{array}{llll}\text { H } & -1.181028 & 0.061833 & -2.154558\end{array}$

C $2.220236 \quad 1.293176-0.813693$

H $2.631929 \quad 2.167220 \quad-1.324244$

H $\quad 2.421416 \quad 1.437673 \quad 0.245047$

C $-2.240646-1.293637-0.970380$

H $\quad-3.085593 \quad-1.206572-1.658588$

H $\quad-2.673111-1.399270 \quad 0.017965$

C $0.714037-1.242879-1.090240$

H $\quad 0.536965 \quad-1.053716 \quad-2.154558$

C $2.240646-1.293637-0.970380$

H $\quad 2.548359-1.615347 \quad 0.017965$

H $\quad 2.587719-2.068916-1.658588$

C $0.009805-2.569369-0.813693$

H $\quad 0.034353 \quad-2.815844 \quad 0.245047$

H $\quad 0.560903 \quad-3.362927 \quad-1.324244$

C $-1.426512-2.539887-1.356606$

H $\quad-1.361246-2.575554-2.448664$

H $-1.964433-3.440221-1.054352$

C $2.9128620 .034548-1.356606$

H $\quad 2.9111180 .108904 \quad-2.448664$

H $3.961535 \quad 0.018862-1.054352$

$\begin{array}{llll}\text { H } & 0.000000 & 0.000000 & 0.644798\end{array}$

\section{H from $3 \mathrm{CBr}_{3}$ :}

Electronic Energy (SCF) $=-508.895971487$ a.u Grimme-D3(BJ) Dispersion energy $=-0.0687094005 \mathrm{a} . \mathrm{u}$

C $\quad 2.586350 \quad 0.010855 \quad 1.564757$

H $3.243818-0.473725 \quad 2.291586$

H $3.057704 \quad-0.133746 \quad 0.600522$

C $2.494162 \quad 1.506353 \quad 1.915940$

H $\quad 2.447962 \quad 1.597490 \quad 3.005564$

H $3.410310 \quad 2.012480 \quad 1.606617$

C $1.238926-0.715295 \quad 1.641613$

$\begin{array}{llll}\text { H } & 0.968811 & -0.653659 & 2.700871\end{array}$

C $\quad 1.265030 \quad 2.232120 \quad 1.349352$

H $\quad 1.369441 \quad 2.432643 \quad 0.285451$

H $\quad 1.177968 \quad 3.206882 \quad 1.835326$

C $\quad 0.000000 \quad 0.000000 \quad 0.983938$

C $\quad 0.000000 \quad 1.430589 \quad 1.641613$

H $\quad 0.081679 \quad 1.165845 \quad 2.700871$ 
$\begin{array}{llll}C & 1.300558 & -2.211608 & 1.349352\end{array}$

H $2.188257-2.623592 \quad 1.835326$

H $\quad 1.422010 \quad-2.402293 \quad 0.285451$

$\begin{array}{llll}\text { C } & -1.302576 & 2.234417 & 1.564757\end{array}$

H $\quad-1.211652 \quad 3.046091 \quad 2.291586$

$\begin{array}{llll}\text { H } & -1.413025 & 2.714923 & 0.600522\end{array}$

C $-1.238926-0.715295 \quad 1.641613$

$\begin{array}{llll}\text { H } & -1.050491 & -0.512186 & 2.700871\end{array}$

C $\quad-1.283774-2.245272 \quad 1.564757$

$\begin{array}{llll}\text { H } & -1.644680 & -2.581177 & 0.600522\end{array}$

$\begin{array}{llll}\text { H } & -2.032167 & -2.572367 & 2.291586\end{array}$

C $\quad-2.565588-0.020512 \quad 1.349352$

$\begin{array}{llll}\text { H } & -2.791451 & -0.030351 & 0.285451\end{array}$

$\begin{array}{llll}\text { H } & -3.366226 & -0.583291 & 1.835326\end{array}$

$\begin{array}{llll}\text { C } & -2.551621 & 1.406832 & 1.915940\end{array}$

$\begin{array}{llll}\text { H } & -2.607448 & 1.321252 & 3.005564\end{array}$

$\begin{array}{llll}\text { H } & -3.448014 & 1.947175 & 1.606617\end{array}$

C $0.057459-2.913184 \quad 1.915940$

$\begin{array}{llll}\text { H } & 0.159486 & -2.918742 & 3.005564\end{array}$

H $\quad 0.037704 \quad-3.959655 \quad 1.606617$

$\begin{array}{llll}\text { H } & 0.000000 & 0.000000 & -0.106062\end{array}$

3H from $3 \mathrm{Cl}_{3}$ :

Electronic Energy (SCF) = -508.892465715 a.u Grimme-D3(BJ) Dispersion energy $=-0.0687381331 \mathrm{a} . \mathrm{u}$

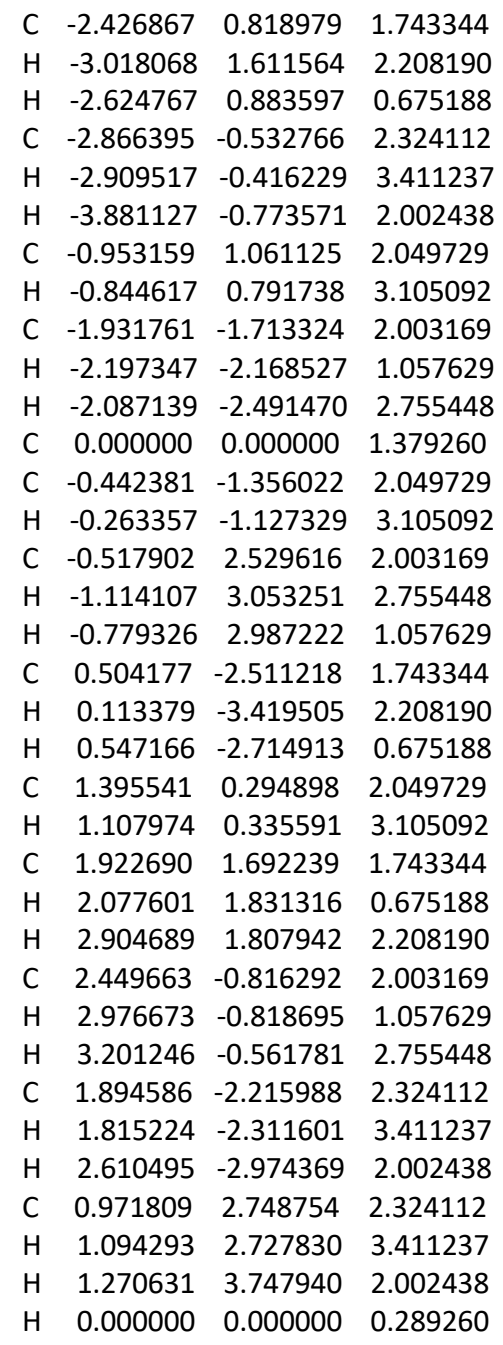

\section{H from 3SiMe :}

Electronic Energy (SCF) = -508.916234415 a.u Grimme-D3(BJ) Dispersion energy $=-0.0692359889 \mathrm{a} . \mathrm{u}$

$\begin{array}{llll}C & 1.276723 & 2.229342 & -0.773795\end{array}$

H $\quad 2.100318 \quad 2.647911-1.358625$

H $\quad 1.503440 \quad 2.455506 \quad 0.263783$

$\begin{array}{llll}\text { C } & -0.032340 & 2.913174 & -1.182337\end{array}$

H $\quad-0.100952 \quad 2.920133-2.274669$

H $\quad-0.021354 \quad 3.958863 \quad-0.867388$

C $1.241843 \quad 0.716979-1.003007$

H $\quad \begin{array}{llll}\text { H.127291 } & 0.588796 & -2.089734\end{array}$

C $\quad-1.272424 \quad 2.199962-0.636348$

H $\quad-1.337573 \quad 2.322797 \quad 0.447768$

H $\quad-2.172921 \quad 2.662540-1.048568$

C $0.000000 \quad 0.000000 \quad-0.399028$

C $-1.241843 \quad 0.716979-1.003007$

H $\quad-1.073558 \quad 0.681865-2.089734$

C $2.541435 \quad 0.001970-0.636348$

H $3.3922880 .550535-1.048568$

$\begin{array}{llll}\text { H } & 2.680388 & -0.003026 & 0.447768\end{array}$

$\begin{array}{llll}\text { C } & -2.569028 & -0.008996 & -0.773795\end{array}$

H $\quad-3.3433180 .494973-1.358625$

$\begin{array}{llll}\text { H } & -2.878250 & 0.074265 & 0.263783\end{array}$

$\begin{array}{llll}\text { C } & 0.000000 & -1.433957 & -1.003007\end{array}$

H $\quad-0.053734-1.270661 \quad-2.089734$

$\begin{array}{llll}\text { C } & 1.292305 & -2.220346 & -0.773795\end{array}$

H $\quad 1.374810-2.529770 \quad 0.263783$

H $\quad 1.242999-3.142885-1.358625$

C $\quad-1.269011-2.201933-0.636348$

H $\quad-1.342814 \quad-2.319771 \quad 0.447768$

H $\quad-1.219367-3.213075-1.048568$

C $-2.506712-1.484594-1.182337$

H $\quad-2.478434 \quad-1.547493 \quad-2.274669$

H $\quad-3.417799-1.997925-0.867388$

C $2.539053-1.428579-1.182337$

H $2.579385-1.372640-2.274669$

H $3.439153 \quad-1.960938-0.867388$

H $\quad 0.000000 \quad 0.000000 \quad 0.690972$

\section{HCCH from 3CCH:}

Electronic Energy (SCF) = -77.3675920383 a.u Grimme-D3(BJ) Dispersion energy $=-0.0022667361 \mathrm{a} . \mathrm{u}$
$\begin{array}{llll}\text { C } & 0.000000 & 0.000000 & 1.496247\end{array}$
$\begin{array}{llll}\text { C } & 0.000000 & 0.000000 & 2.696841\end{array}$
H $\quad 0.000000 \quad 0.000000 \quad 3.757990$
$\begin{array}{llll}H & 0.000000 & 0.000000 & 0.416247\end{array}$

\section{HCN from $3 \mathrm{CN}$ :}

Electronic Energy (SCF) = -93.4659370738 a.u Grimme-D3(BJ) Dispersion energy $=-0.0014547565$ a.u

$$
\begin{array}{llll}
\mathrm{C} & 0.000000 & 0.000000 & 1.483007 \\
\mathrm{~N} & 0.000000 & 0.000000 & 2.633566 \\
\mathrm{H} & 0.000000 & 0.000000 & 0.403007
\end{array}
$$

$\mathrm{CH}_{4}$ from 3Me:

Electronic Energy (SCF) = -40.5404209448 a.u 
Grimme-D3(BJ) Dispersion energy $=-0.0019136090 \mathrm{a} . \mathrm{u}$

$\begin{array}{rrrr}\text { C } & 0.000000 & 0.000000 & 1.750431 \\ \text { H } & 0.000000 & 1.009666 & 2.153613 \\ \text { H } & -0.874396 & -0.504833 & 2.153613 \\ \text { H } & 0.874396 & -0.504833 & 2.153613 \\ \text { H } & 0.000000 & 0.000000 & 0.660431\end{array}$

\section{Ethane from 3Et:}

Electronic Energy (SCF) $=-79.8677384115$ a.u Grimme-D3(BJ) Dispersion energy $=-0.0054058229$ a.u

$\begin{array}{cccc}\text { C } & -0.037350 & 0.030201 & 1.633328 \\ \text { H } & -0.570725 & 0.921870 & 1.959535 \\ \text { H } & -0.646550 & -0.798144 & 1.989631 \\ \text { C } & 1.270401 & 0.004216 & 2.430519 \\ \text { H } & 1.873473 & 0.895518 & 2.268467 \\ \text { H } & 1.027814 & -0.027803 & 3.494617 \\ \text { H } & 1.892976 & -0.860717 & 2.216171 \\ \text { H } & -0.056233 & 0.009344 & 0.543691\end{array}$

Propane from 3iPr:

Electronic Energy (SCF) = -119.197906292 a.u Grimme-D3(BJ) Dispersion energy $=-0.0100683091 \mathrm{a} . \mathrm{u}$

C $0.036852 \quad-0.012082 \quad 1.575322$

H $\quad 0.030659 \quad 1.030667 \quad 1.892037$

$\begin{array}{llll}\text { C } & 1.281511 & -0.614829 & 2.252684\end{array}$

H $\quad \begin{array}{llll}1.388083 & -1.679817 & 2.053191\end{array}$

H $\quad 2.209126-0.125847 \quad 1.977500$

H $\quad \begin{array}{llll}\text { H.170081 } & -0.501844 & 3.332588\end{array}$

C $\quad-1.157503 \quad-0.666596 \quad 2.293223$

H $\quad-1.155448 \quad-1.748994 \quad 2.165386$

H $\quad-1.063884-0.473395 \quad 3.363321$

H $\quad-2.126358 \quad-0.294622 \quad 1.983924$

H $\quad 0.0088320 .033066 \quad 0.486618$

Iso-Butane from $3 \mathrm{tBu}$ :

Electronic Energy (SCF) = -158.531527156 a.u Grimme-D3(BJ) Dispersion energy $=-0.0158725878$ a.u

$\begin{array}{llll}\text { C } & 0.000000 & 0.000000 & 1.544818\end{array}$

C $1.230920-0.685798 \quad 2.176580$

H $\quad 1.291030-1.742892 \quad 1.934015$

H $\quad 2.169774-0.215555 \quad 1.901455$

H $\quad \begin{array}{llll}1.140978 & -0.611831 & 3.261498\end{array}$

C $-1.209378-0.723109 \quad 2.176580$

H $\quad-1.271563 \quad-1.771302 \quad 1.901455$

H $-1.100350-0.6822013 .261498$

H $\quad-2.154903 \quad-0.246619 \quad 1.934015$

C $\quad-0.021542 \quad 1.408907 \quad 2.176580$

H $\quad-0.040628 \quad 1.294032 \quad 3.261498$

H $\quad 0.863874 \quad 1.989510 \quad 1.934015$

H $\quad-0.898211 \quad 1.986857 \quad 1.901455$

$\begin{array}{llll}\text { H } & 0.000000 & 0.000000 & 0.454818\end{array}$

Ph from 3Ph:

Electronic Energy (SCF) $=-232.356366665$ a.u Grimme-D3(BJ) Dispersion energy $=-0.0189502914$ a.u

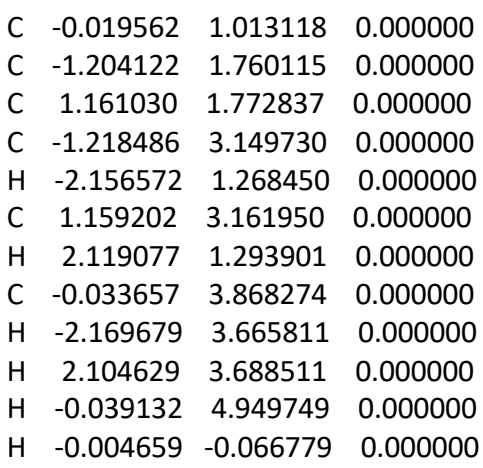

$\mathrm{HCF}_{3}$ from $3 \mathrm{CF}_{3}$ :

Electronic Energy (SCF) $=-338.393399943$ a.u Grimme-D3(BJ) Dispersion energy $=-0.0026771922$ a.u

$\begin{array}{crrr}\text { C } & 0.000000 & 0.000000 & 1.401249 \\ \text { F } & 0.000000 & 1.241349 & 1.941048 \\ \text { F } & -1.075039 & -0.620674 & 1.941048 \\ \text { F } & 1.075039 & -0.620674 & 1.941048 \\ \text { H } & 0.000000 & 0.000000 & 0.311249\end{array}$

$\mathrm{HCCl}_{3}$ from $3 \mathrm{CCl}_{3}$ :

Electronic Energy (SCF) = -1419.39034974 a.u Grimme-D3(BJ) Dispersion energy $=-0.0103556736 \mathrm{a} . \mathrm{u}$

C $\quad 0.000000 \quad 0.000000 \quad 1.142778$

$\mathrm{Cl} \quad-0.502333 \quad 1.564563 \quad 1.903986$

$\begin{array}{llll}\text { Cl } & 1.606118 & -0.347248 & 1.903986\end{array}$

Cl $-1.103785-1.217315 \quad 1.903986$

H $\quad 0.000000 \quad 0.000000 \quad 0.052778$

$\mathrm{HCBr}_{3}$ from $3 \mathrm{CBr}_{3}$ :

Electronic Energy (SCF) $=-7761.25054588$ a.u Grimme-D3(BJ) Dispersion energy $=-0.0131084523 \mathrm{a} . \mathrm{u}$

C $\quad 0.000000 \quad 0.000000 \quad-0.604942$

$\begin{array}{lllll}\mathrm{Br} & -1.250441 & 1.284166 & -1.483254\end{array}$

$\mathrm{Br} \quad-0.486900-1.724997-1.483254$

$\begin{array}{llll}\mathrm{Br} & 1.737341 & 0.440831 & -1.483254\end{array}$

$\begin{array}{llll}\text { H } & 0.000000 & 0.000000 & 0.485058\end{array}$

$\mathrm{HCl}_{3}$ from $3 \mathrm{Cl}_{3}$ :

Electronic Energy (SCF) $=-932.102717023$ a.u

Grimme-D3(BJ) Dispersion energy $=-0.0179321424$ a.u

C $\quad 0.000000 \quad 0.000000 \quad-0.213097$

I $0.000000 \quad 1.979363 \quad-1.250371$

I $1.714179-0.989681-1.250371$

I $\quad-1.714179-0.989681-1.250371$

H $\quad 0.000000 \quad 0.000000 \quad 0.876903$

$\mathrm{HSiMe}_{3}$ from $3 \mathrm{SiMe}_{3}$

Electronic Energy (SCF) = -409.963496342 a.u Grimme-D3(BJ) Dispersion energy $=-0.0181072925$ a.u 
$\begin{array}{llll}\text { C } & 0.891251 & -1.459025 & 2.374979\end{array}$

H $\quad 0.430201-2.421471 \quad 2.156972$

$\begin{array}{llll}\text { H } & 1.947729 & -1.522294 & 2.118927\end{array}$

$\begin{array}{llll}\text { H } & 0.825174 & -1.304591 & 3.455010\end{array}$

$\begin{array}{llll}\text { C } & -1.709178 & -0.042333 & 2.374979\end{array}$

$\begin{array}{llll}\text { H } & -2.292210 & -0.925636 & 2.118927\end{array}$

$\begin{array}{llll}\text { H } & -1.542396 & -0.062326 & 3.455010\end{array}$

$\begin{array}{llll}\text { H } & -2.312156 & 0.838170 & 2.156972\end{array}$

$\begin{array}{llll}\text { C } & 0.817927 & 1.501358 & 2.374979\end{array}$

$\begin{array}{llll}\text { H } & 0.717222 & 1.366917 & 3.455010\end{array}$

H $\quad 1.881955 \quad 1.583300 \quad 2.156972$

$\begin{array}{cccc}\mathrm{H} & 0.344480 & 2.447930 & 2.118927\end{array}$

Si $\quad 0.000000 \quad 0.000000 \quad 1.568065$

H $\quad 0.000000 \quad 0.000000 \quad 0.088065$

\section{Cyclohexanes with Boron substituents:}

\section{Bglyax (B3LYP-D3BJ/def2-TZVPP, 183 K, DCM):}

Imaginary frequencies- 0

Electronic Energy (SCF) = -489.539532807 a.u Grimme-D3(BJ) Dispersion energy $=-0.0435742080 \mathrm{a} . \mathrm{u}$ ZPV corrected Energy = -489.309782 a.u.

Enthalpy $=-489.304675$ a.u.

Free Energy $=-489.329579$ a.u.

Free Energy after quasiharmonic app. $=-489.328653$ a.u.

$\begin{array}{llll}\text { C } & -0.524144 & 0.033768 & 1.124936\end{array}$

C $-1.349969-1.235952 \quad 0.849763$

$\begin{array}{llll}\text { H } & -2.213426 & -1.254153 & 1.523297\end{array}$

$\begin{array}{llll}\text { H } & -0.759077 & -2.127026 & 1.068578\end{array}$

C $\quad-1.344693 \quad 1.289106 \quad 0.776346$

$\begin{array}{llll}\text { H } & -0.749770 & 2.188875 & 0.942266\end{array}$

$\begin{array}{llll}\text { H } & -2.206917 & 1.349950 & 1.448906\end{array}$

$\begin{array}{llll}\text { C } & -1.847661 & 1.249502 & -0.668356\end{array}$

$\begin{array}{lllll}\mathrm{H} & -2.454683 & 2.133213 & -0.879122\end{array}$

H $\quad-0.990173 \quad 1.288906 \quad-1.348266$

$\begin{array}{llll}\text { C } & -1.850291 & -1.277682 & -0.595612\end{array}$

$\begin{array}{llll}\mathrm{H} & -0.991227 & -1.356666 & -1.270259\end{array}$

$\begin{array}{llll}\text { H } & -2.458240 & -2.170988 & -0.757521\end{array}$

C $-2.651091-0.022318-0.947012$

$\begin{array}{llll}\text { H } & -2.960891 & -0.051978 & -1.994441\end{array}$

$\begin{array}{llll}\mathrm{H} & -3.568105 & -0.004322 & -0.348162\end{array}$

$\begin{array}{llll}H & -0.300753 & 0.065231 & 2.200311\end{array}$

$\begin{array}{llll}\text { B } & 0.886327 & 0.010607 & 0.431349\end{array}$

$\begin{array}{lllll}\text { O } & 1.580994 & 1.142032 & 0.075469\end{array}$

$\begin{array}{llll}\text { O } & 1.585361 & -1.142101 & 0.162599\end{array}$

$\begin{array}{llll}\text { C } & 2.893311 & 0.743897 & -0.371546\end{array}$

$\begin{array}{llll}\text { C } & 2.804642 & -0.782187 & -0.518698\end{array}$

$\begin{array}{llll}\text { H } & 3.642174 & -1.300912 & -0.055968\end{array}$

H $\quad 2.724072-1.094069-1.560687$

H $\quad 3.120879 \quad 1.246723 \quad-1.309714$

H $\quad 3.623039 \quad 1.0459330 .380552$

\section{Bglyeq $1^{\text {st }}$ (B3LYP-D3BJ/def2-TZVPP, 183 K, DCM):}

Imaginary frequencies- 0

Electronic Energy (SCF) = -489.539517438 a.u

Grimme-D3(BJ) Dispersion energy $=-0.0420876419$ a.u ZPV corrected Energy = -489.309797 a.u.

Enthalpy $=-489.304570$ a.u.

Free Energy = -489.329974 a.u.

Free Energy after quasiharmonic app. $=-489.328782$ a.u.

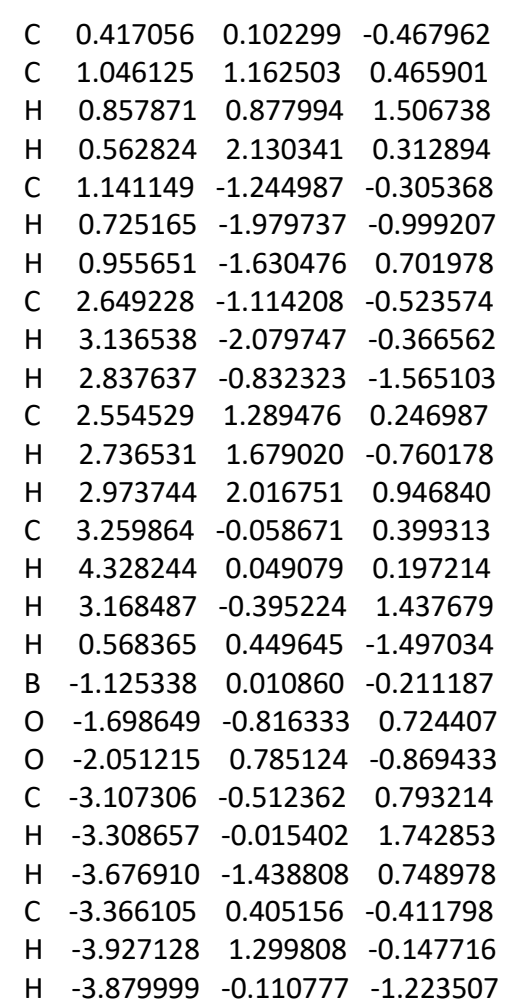

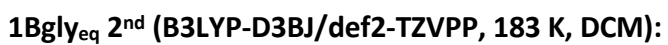
Imaginary frequencies- 0

Electronic Energy (SCF) $=-489.539559475$ a.u Grimme-D3(BJ) Dispersion energy $=-0.0421005671$ a.u ZPV corrected Energy $=-489.309928$ a.u.

Enthalpy $=-489.304702$ a.u.

Free Energy = -489.330202 a.u.

Free Energy after quasiharmonic app. $=-489.328916$ a.u.

$\begin{array}{lrrr}\text { C } & 0.418308 & 0.085870 & 0.462566 \\ \text { C } & 1.147764 & -1.253840 & 0.274729 \\ \text { H } & 0.963037 & -1.621637 & -0.739447 \\ \text { H } & 0.735088 & -2.003395 & 0.954394 \\ \text { C } & 1.042365 & 1.168832 & -0.448420 \\ \text { H } & 0.554765 & 2.130573 & -0.273990 \\ \text { H } & 0.854668 & 0.906347 & -1.495324 \\ \text { C } & 2.550438 & 1.296899 & -0.228282 \\ \text { H } & 2.966436 & 2.039947 & -0.913346 \\ \text { H } & 2.731497 & 1.666411 & 0.786611 \\ \text { C } & 2.655557 & -1.121244 & 0.493937 \\ \text { H } & 2.843978 & -0.859018 & 1.540642 \\ \text { H } & 3.146993 & -2.081344 & 0.317619 \\ \text { C } & 3.260953 & -0.045088 & -0.408378 \\ \text { H } & 4.329156 & 0.062926 & -0.205455 \\ \text { H } & 3.169674 & -0.361131 & -1.453200 \\ \text { H } & 0.568848 & 0.415719 & 1.498222 \\ B & -1.125708 & 0.002314 & 0.212781 \\ \text { O } & -1.725686 & -0.913244 & -0.617326 \\ \text { O } & -2.027227 & 0.876318 & 0.774071 \\ \text { C } & -3.153268 & -0.711398 & -0.555396 \\ \text { H } & -3.599201 & -1.551529 & -0.021913 \\ \text { H } & -3.555094 & -0.678276 & -1.566403 \\ \text { C } & -3.324233 & 0.616383 & 0.197689 \\ \text { H } & -4.065848 & 0.559089 & 0.992368\end{array}$


H $\quad-3.579454 \quad 1.440662-0.469315$

1Bglyeq $3^{\text {rd }}$ (B3LYP-D3BJ/def2-TZVPP, 183 K, DCM): Imaginary frequencies- 0

Electronic Energy (SCF) = -489.539464386 a.u Grimme-D3(BJ) Dispersion energy $=-0.0420764876 \mathrm{a} . \mathrm{u}$ ZPV corrected Energy = -489.309921 a.u.

Enthalpy $=-489.304727$ a.u.

Free Energy = -489.330099 a.u.

Free Energy after quasiharmonic app. $=-489.328881$ a.u.

C $0.418376 \quad 0.016336 \quad-0.405552$

$\begin{array}{llll}\text { C } & 1.101444 & 1.254252 & 0.205094\end{array}$

H $\quad 0.944855 \quad 1.249011 \quad 1.289486$

H $\quad 0.631281 \quad 2.164639-0.170805$

C $\quad 1.118494 \quad-1.273553 \quad 0.053926$

H $0.660836-2.140058-0.426998$

H $\quad 0.962420 \quad-1.399841 \quad 1.130826$

C $2.620351-1.242930-0.232957$

$\begin{array}{llll}\text { H } & 3.093725 & -2.153614 & 0.142598\end{array}$

H $\quad 2.776282 \quad-1.231338-1.317213$

C $2.603332 \quad 1.278106-0.081795$

H $\quad 2.758297 \quad 1.398511-1.159486$

H $\quad 3.064283 \quad 2.143682 \quad 0.400714$

$\begin{array}{llll}\text { C } & 3.285267 & -0.010148 & 0.380943\end{array}$

H $\quad 4.3482390 .012303 \quad 0.128846$

H $3.222566 \quad-0.075996 \quad 1.472645$

H $\quad 0.546183 \quad 0.085856-1.496985$

B $\quad-1.130792 \quad 0.001957 \quad-0.164249$

$\begin{array}{llll}\text { O } & -1.872374 & -1.143448 & -0.001128\end{array}$

$\begin{array}{llll}0 & -1.901137 & 1.139793 & -0.115345\end{array}$

C $\quad-3.238301-0.764165 \quad 0.267053$

H $-3.464115-0.996975 \quad 1.308175$

H $\quad-3.903130 \quad-1.336372-0.377456$

$\begin{array}{llll}\text { C } & -3.284987 & 0.744813 & -0.017947\end{array}$

H $\quad-3.764338 \quad 1.310400 \quad 0.779044$

H $\quad-3.7792010 .972983 \quad-0.962965$

1Bpin $_{\text {ax }}$ (B3LYP-D3BJ/def2-TZVPP, 183 K, DCM): Imaginary frequencies- 0

Electronic Energy (SCF) = -646.897892635 a.u

Grimme-D3(BJ) Dispersion energy $=-0.0711641158$ a.u

ZPV corrected Energy = -646.557403 a.u.

Enthalpy $=-646.550611$ a.u.

Free Energy $=-646.579022$ a.u.

Free Energy after quasiharmonic app. $=-646.507960$ a.u.

C $-1.562875-0.310420-1.180602$

C $-2.374972 \quad 0.995884-1.128061$

H $\quad-3.2839120 .879056-1.728106$

H $\quad-1.804938 \quad 1.813449 \quad-1.572858$

C $-2.348344-1.454265-0.513969$

H $\quad-1.759494-2.373105-0.523648$

H $\quad-3.255970-1.652383-1.094263$

C $\quad-2.747144 \quad-1.1005390 .920447$

H $\quad-3.331803 \quad-1.912608 \quad 1.359437$

H $\quad-1.842898-1.000651 \quad 1.529503$

C $\quad-2.772611 \quad 1.352112 \quad 0.305931$

$\begin{array}{llll}\text { H } & -1.869184 & 1.568590 & 0.885314\end{array}$

H $\quad-3.374782 \quad 2.2638890 .313846$

C $\quad-3.537088 \quad 0.208545 \quad 0.975689$

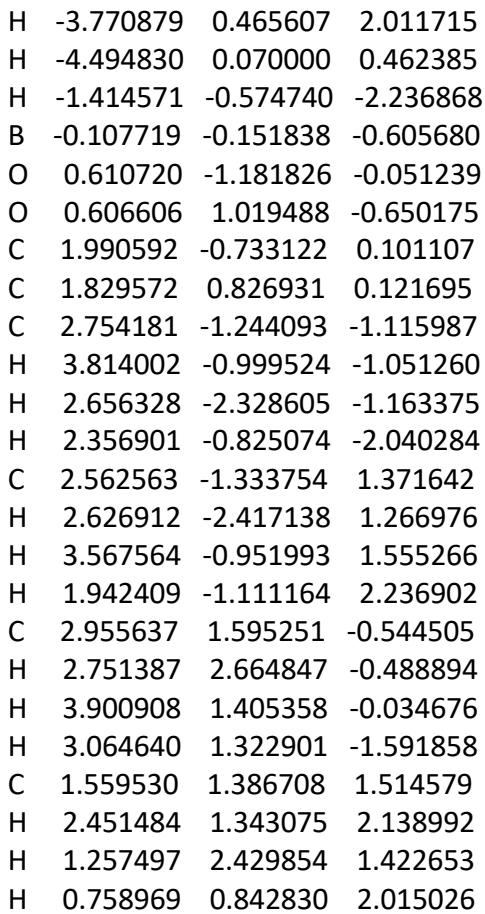

$1_{\text {Bpin }} 1^{\text {st }}$ (B3LYP-D3BJ/def2-TZVPP, $\left.183 \mathrm{~K}, \mathrm{DCM}\right)$ :

Imaginary frequencies- 0

Electronic Energy (SCF) = -646.897581357 a.u

Grimme-D3(BJ) Dispersion energy $=-0.0691076914$ a.u

ZPV corrected Energy $=-646.557182$ a.u.

Enthalpy $=-646.550259$ a.u.

Free Energy = -646.579473 a.u.

Free Energy after quasiharmonic app. $=-646.509540$ a.u.

$\begin{array}{llll}\text { C } & 1.498989 & 0.161024 & -0.526286\end{array}$

C $\quad 2.105361 \quad 1.121347 \quad 0.523317$

H $\quad 1.883132 \quad 0.740076 \quad 1.525940$

H $\quad 1.631751 \quad 2.103151 \quad 0.449557$

C $2.212710-1.200059-0.475401$

H $\quad 1.815018-1.860276-1.250261$

$\begin{array}{llll}\text { H } & 1.993934 & -1.681458 & 0.482811\end{array}$

C $3.727557-1.060823-0.633234$

H $\quad 4.205278-2.040817-0.556420$

H $3.950299-0.679796-1.635740$

C $3.620589 \quad 1.256973 \quad 0.364688$

H $3.836339 \quad 1.740678-0.593842$

H $\quad 4.021487 \quad 1.909637 \quad 1.144372$

C $4.313796 \quad-0.105336 \quad 0.407044$

H $\quad \begin{array}{llll}\text { H } & 5888738 & 0.012628 & 0.250425\end{array}$

H $4.186955-0.540511 \quad 1.404247$

H $\quad \begin{array}{llll}1.679263 & 0.606122 & -1.512241\end{array}$

B $\quad-0.049549 \quad 0.056351 \quad-0.311329$

$\begin{array}{llll}\text { O } & -0.647384 & -0.879664 & 0.495024\end{array}$

$\begin{array}{lllll}0 & -0.955036 & 0.926289 & -0.866751\end{array}$

$\begin{array}{llll}\text { C } & -2.037894 & -0.479528 & 0.684998\end{array}$

$\begin{array}{llll}\text { C } & -2.294739 & 0.428956 & -0.567652\end{array}$

$\begin{array}{llll}\text { C } & -2.094534 & 0.283887 & 2.004132\end{array}$

$\begin{array}{llll}\text { H } & -3.115403 & 0.572710 & 2.252125\end{array}$

$\begin{array}{llll}\text { H } & -1.718666 & -0.359312 & 2.799719\end{array}$

H $-1.477403 \quad 1.181561 \quad 1.971576$

C $-2.900343 \quad-1.7254310 .760425$

H $\quad-2.643668-2.293785 \quad 1.654800$ 


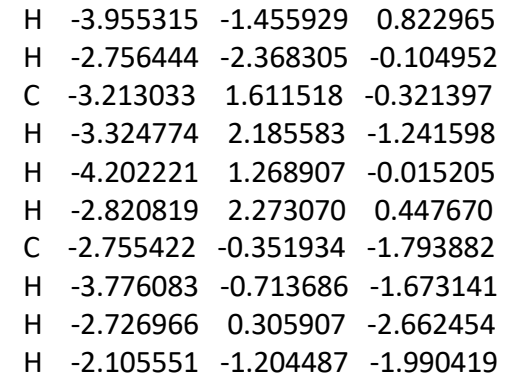

1Bpin $_{\text {eq }} 2^{\text {nd }}$ (B3LYP-D3BJ/def2-TZVPP, $\left.183 \mathrm{~K}, \mathrm{DCM}\right)$ : Imaginary frequencies- 1

Electronic Energy (SCF) = -646.897697073 a.u Grimme-D3(BJ) Dispersion energy $=-0.0691458473 \mathrm{a} . \mathrm{u}$ ZPV corrected Energy $=-646.557513$ a.u. Enthalpy $=-646.551109$ a.u.

Free Energy $=-646.578447$ a.u.

Free Energy after quasiharmonic app. $=-646.509666$ a.u.

$\begin{array}{llll}C & -1.502512 & 0.082354 & -0.518536\end{array}$

$\begin{array}{llll}\text { C } & -2.221003 & -1.256444 & -0.289577\end{array}$

H $\quad-2.003838 \quad-1.608574 \quad 0.723761$

H $\quad-1.825237-2.014518-0.970023$

C $\quad-2.103981 \quad 1.176601 \quad 0.393701$

H $-1.624985 \quad 2.137141 \quad 0.190713$

H $\quad-1.8841590 .930807 \quad 1.438454$

C $\quad-3.618510 \quad 1.296235 \quad 0.217415$

H $\quad-4.017273 \quad 2.047850 \quad 0.903481$

H $\quad-3.831729 \quad 1.649585-0.797009$

C $-3.735336-1.133375-0.464895$

H $\quad-3.956531-0.887686-1.509222$

H $-4.217586-2.092366-0.259251$

C $-4.317566 \quad-0.045709 \quad 0.439012$

H $\quad-5.391951 \quad 0.055117 \quad 0.267242$

H $\quad-4.193080-0.345453 \quad 1.485227$

H $\quad-1.682791 \quad 0.396283 \quad-1.554505$

$\begin{array}{llll}\text { B } & 0.047971 & 0.009767 & -0.305956\end{array}$

$\begin{array}{lllll}\text { O } & 0.668274 & -0.862397 & 0.552349\end{array}$

$\begin{array}{lllll}0 & 0.934515 & 0.855438 & -0.926981\end{array}$

C $2.109145 \quad-0.732034 \quad 0.358551$

$\begin{array}{llll}\text { C } & 2.229091 & 0.699732 & -0.270807\end{array}$

$\begin{array}{llll}\text { C } & 2.524081 & -1.847313 & -0.595154\end{array}$

H $3.602632-1.856621 \quad-0.749363$

H $\quad 2.231938-2.805418-0.165456$

H $\quad 2.035433 \quad-1.745010-1.563916$

C $2.800270-0.900575 \quad 1.698684$

H $\quad 2.653404-1.918515 \quad 2.060586$

H $\quad 3.872768 \quad-0.729392 \quad 1.598704$

H $\quad 2.406607 \quad-0.213220 \quad 2.444016$

C $3.3267630 .853992 \quad-1.306730$

H $\quad 3.325920 \quad 1.874313 \quad-1.691096$

H $4.302903 \quad 0.663070-0.859239$

$\begin{array}{llll}\text { H } & 3.189321 & 0.173845 & -2.144210\end{array}$

C $2.326191 \quad 1.808257 \quad 0.772273$

H $3.294309 \quad 1.799554 \quad 1.272103$

H $\quad 2.206729 \quad 2.771022 \quad 0.275539$

H $\quad 1.545236 \quad 1.714705 \quad 1.526681$

1Bpin $_{\text {eq }} 3^{\text {rd }}$ (B3LYP-D3BJ/def2-TZVPP, $\left.183 \mathrm{~K}, \mathrm{DCM}\right)$ : Imaginary frequencies- 0
Electronic Energy (SCF) $=-646.897494824$ a.u Grimme-D3(BJ) Dispersion energy $=-0.0689554833$ a.u ZPV corrected Energy = -646.557299 a.u.

Enthalpy $=-646.550403$ a.u.

Free Energy = -646.579497 a.u.

Free Energy after quasiharmonic app. $=-646.509747$ a.u.

C $\quad-1.504419-0.075069-0.439150$

C $-2.179879-1.233179 \quad 0.316865$

H $\quad-2.004909-1.107558 \quad 1.391172$

H $\quad-1.717664-2.181804 \quad 0.038055$

C $-2.193918 \quad 1.261252-0.115710$

H $\quad-1.741968 \quad 2.066065 \quad-0.698378$

H $\quad-2.017957 \quad 1.506651 \quad 0.937479$

$\begin{array}{llll}\text { C } & -3.700752 & 1.205790 & -0.370852\end{array}$

H $\quad-4.165632 \quad 2.154498-0.090740$

H $\quad-3.875980 \quad 1.074258 \quad-1.444212$

$\begin{array}{llll}\text { C } & -3.686557 & -1.283129 & 0.059923\end{array}$

H $\quad-3.860452-1.521750-0.994999$

H $\quad-4.141672 \quad-2.087623 \quad 0.643261$

C $\quad-4.357500 \quad 0.051578 \quad 0.387736$

$\begin{array}{llll}\text { H } & -5.424850 & 0.005732 & 0.158107\end{array}$

$\begin{array}{llll}\mathrm{H} & -4.275507 & 0.238301 & 1.464094\end{array}$

H $\quad-1.650484-0.264627 \quad-1.514139$

B $\quad 0.049083 \quad-0.034944 \quad-0.225754$

$\begin{array}{lllll}\text { O } & 0.802515 & 1.110104 & -0.299852\end{array}$

$\begin{array}{llll}0 & 0.810844 & -1.147114 & 0.035079\end{array}$

C $2.153466 \quad 0.786470 \quad 0.146665$

$\begin{array}{llll}\text { C } & 2.214220 & -0.764532 & -0.080467\end{array}$

C $2.233456 \quad 1.184169 \quad 1.616868$

H $3.234903 \quad 1.0275592 .016596$

H $1.990490 \quad 2.242666 \quad 1.709109$

$\begin{array}{llll}\text { H } & 1.525284 & 0.618068 & 2.221629\end{array}$

C $3.146161 \quad 1.592096-0.670506$

H $3.022142 \quad 2.653356-0.453411$

H $\quad 4.168407 \quad 1.312263 \quad-0.413164$

H $\quad 3.003510 \quad 1.443781 \quad-1.738455$

C $3.017270 \quad-1.5312420 .953764$

H $2.995189-2.5956090 .718300$

H $4.058106-1.2055210 .946580$

H $2.617489-1.395649 \quad 1.956098$

C 2.659365 -1.150159 -1.487257

H $3.719181-0.946509-1.637393$

H $2.492972 \quad-2.217866-1.629714$

H $\quad 2.091637 \quad-0.612141 \quad-2.246189$

1Bglyax (B3LYP/def2-TZVPP, 183 K, DCM):

Imaginary frequencies- 0

Electronic Energy (SCF) $=-489.496158517$ a.u

ZPV corrected Energy $=-489.266815$ a.u.

Enthalpy $=-489.261659$ a.u.

Free Energy $=-489.286668$ a.u.

Free Energy after quasiharmonic app. $=-489.285713$ a.u.

$\begin{array}{llll}\text { C } & -0.507705 & 0.017847 & 1.094269\end{array}$

C $-1.342019-1.251421 \quad 0.824362$

H $\quad-2.175277 \quad-1.286899 \quad 1.535026$

H $\quad-0.741185-2.145575 \quad 1.002364$

C $\quad-1.338966 \quad 1.279960 \quad 0.783967$

$\begin{array}{llll}\text { H } & -0.735901 & 2.177899 & 0.933001\end{array}$

H $\quad-2.171466 \quad 1.339917 \quad 1.493855$

C $\quad-1.907444 \quad 1.258495 \quad-0.638908$ 


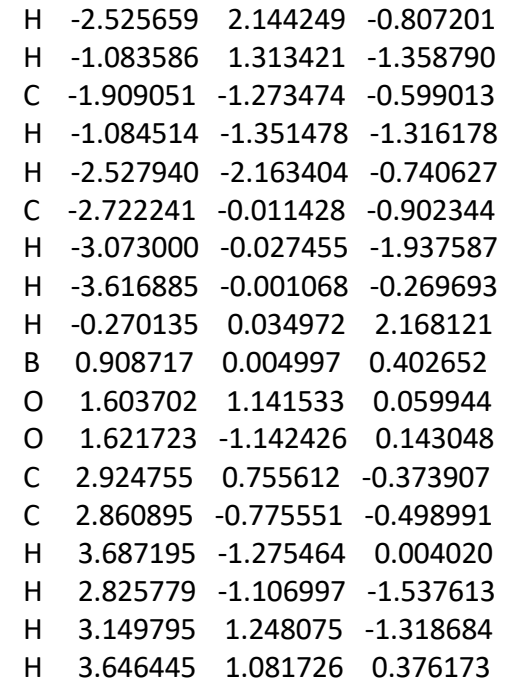

1Bglyeq $1^{\text {st }}(B 3 L Y P /$ def2-TZVPP, $183 \mathrm{~K}, \mathrm{DCM})$ : Imaginary frequencies- 0

Electronic Energy (SCF) $=-489.497510488$ a.u ZPV corrected Energy $=-489.268125$ a.u.

Enthalpy $=-489.262883$ a.u.

Free Energy $=-489.288280$ a.u.

Free Energy after quasiharmonic app. $=-489.287108$ a.u.

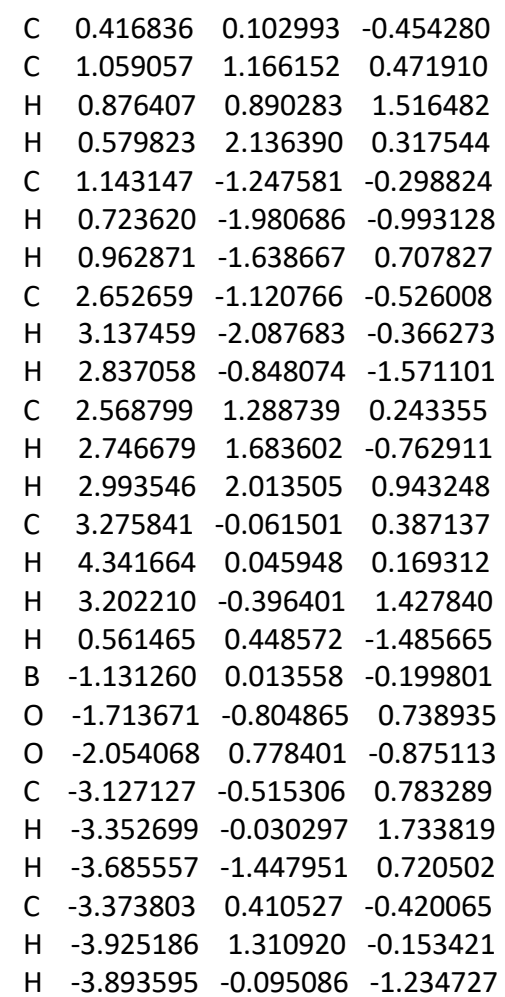

1Bglyeq $2^{\text {nd }}(B 3 L Y P / d e f 2-T Z V P P, 183$ K, DCM): Imaginary frequencies- 0

Electronic Energy (SCF) $=-489.497538962$ a.u

ZPV corrected Energy $=-489.268245$ a.u.

Enthalpy $=-489.262976$ a.u.

Free Energy $=-489.289462$ a.u.

Free Energy after quasiharmonic app. $=-489.287205$ a.u.

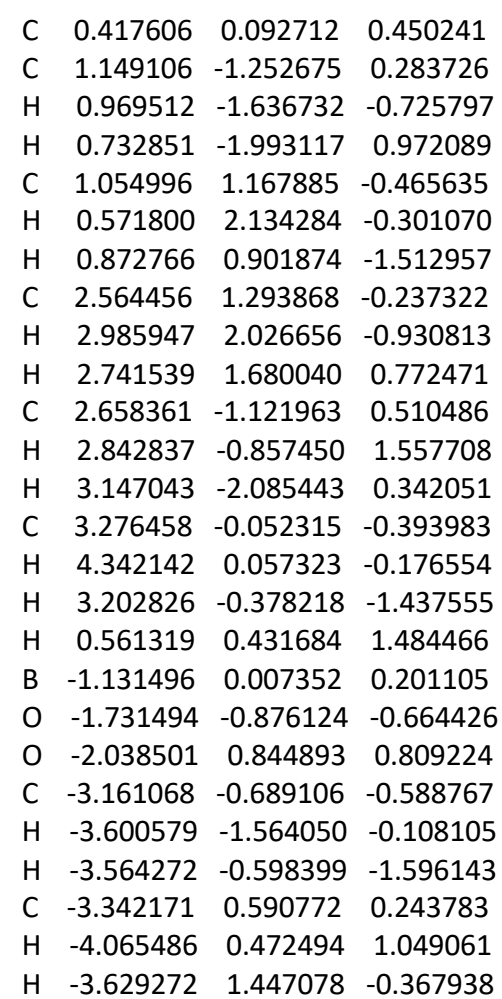

1Bgly ${ }_{\text {eq }} 3^{\text {rd }}$ (B3LYP/def2-TZVPP, 183 K, DCM): Imaginary frequencies- 0

Electronic Energy (SCF) $=-489.497458739$ a.u ZPV corrected Energy $=-489.268294$ a.u. Enthalpy $=-489.263063$ a.u.

Free Energy = -489.288537 a.u.

Free Energy after quasiharmonic app. $=-489.287236$ a.u.

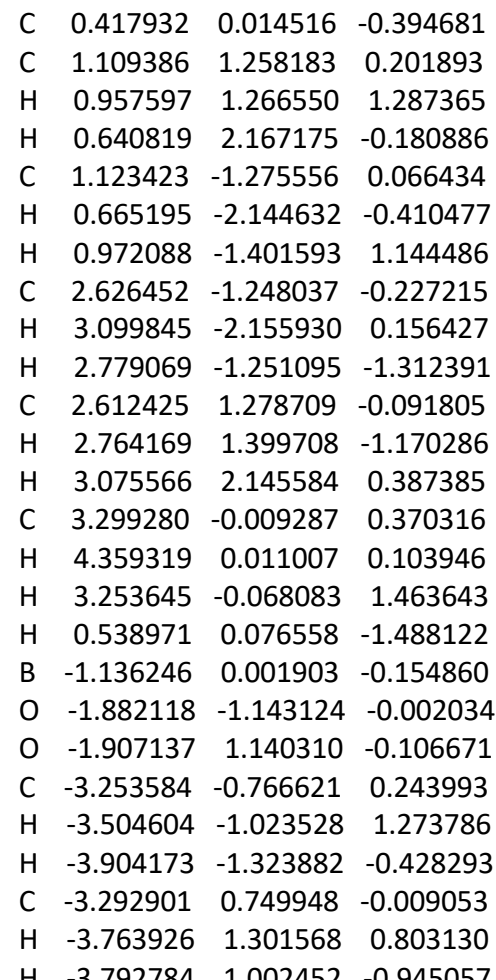

$\begin{array}{llll}H & -3.792784 & 1.002452 & -0.945057\end{array}$ 
1Bpin ${ }_{\mathrm{ax}}$ (B3LYP/def2-TZVPP, $\left.183 \mathrm{~K}, \mathrm{DCM}\right)$ :

Imaginary frequencies- 0

Electronic Energy (SCF) $=-646.827239465$ a.u ZPV corrected Energy = -646.487465 a.u.

Enthalpy $=-646.480611$ a.u.

Free Energy = -646.509256 a.u.

Free Energy after quasiharmonic app. $=-646.507960$ a.u.

C $-1.553685-0.264901-1.140643$

C $-2.382961 \quad 1.034590-1.089843$

H $\quad-3.250438 \quad 0.929924-1.751313$

H $\quad-1.797616 \quad 1.872897 \quad-1.473211$

$\begin{array}{llll}\text { C } & -2.356514 & -1.436097 & -0.537672\end{array}$

H $-1.753005-2.346023-0.531632$

H $\quad-3.223702-1.639509-1.176041$

C $-2.853093 \quad-1.1251280 .878229$

H $\quad-3.453274-1.957804 \quad 1.254626$

H $\quad-1.994123 \quad-1.032138 \quad 1.551535$

C $\quad-2.880858 \quad 1.345503 \quad 0.325583$

H $\quad-2.023737 \quad 1.566760 \quad 0.970646$

$\begin{array}{llll}\text { H } & -3.500547 & 2.246111 & 0.313984\end{array}$

C $\quad-3.666535 \quad 0.1720350 .918588$

H $\quad-3.9666650 .398387 \quad 1.945141$

$\begin{array}{llll}H & -4.590837 & 0.033545 & 0.346473\end{array}$

$\begin{array}{llll}\text { H } & -1.376618 & -0.500742 & -2.200677\end{array}$

B $-0.095455-0.121689-0.554342$

$\begin{array}{llll}\text { O } & 0.629808 & -1.170815 & -0.043023\end{array}$

\begin{tabular}{lllll}
\hline & 0.627735 & 1.046105 & -0.578639
\end{tabular}

C $2.020079-0.743985 \quad 0.093211$

$\begin{array}{llll}\text { C } & 1.884714 & 0.826323 & 0.132623\end{array}$

C $2.759266-1.262160-1.139901$

H $3.823842 \quad-1.032432-1.092815$

H $\quad 2.648267-2.345864 \quad-1.188400$

H $\quad 2.354642 \quad-0.838839-2.059419$

C $2.599648-1.374476 \quad 1.350176$

H $\quad 2.627367 \quad-2.458722 \quad 1.234313$

H $3.620785-1.029467 \quad 1.519215$

H $\quad 2.005359-1.143300 \quad 2.231776$

C $2.987012 \quad 1.588051-0.587706$

H $\quad 2.789424 \quad 2.659055-0.526815$

H $\quad 3.954983 \quad 1.399230 \quad-0.121094$

H $3.049759 \quad 1.314675-1.639010$

C $\quad 1.697514 \quad 1.387826 \quad 1.541423$

$\begin{array}{llll}\text { H } & 2.616478 & 1.316586 & 2.123498\end{array}$

H $\quad 1.423052 \quad 2.440661 \quad 1.468983$

H $\quad 0.906024 \quad 0.867668 \quad 2.080831$

\section{$1_{\text {Bpin }} 1^{\text {st }}(B 3 L Y P /$ def2-TZVPP, $183 \mathrm{~K}, \mathrm{DCM}):$}

Imaginary frequencies- 0

Electronic Energy (SCF) $=-646.828727530$ a.u

ZPV corrected Energy = -646.489095 a.u.

Enthalpy $=-646.482105$ a.u.

Free Energy $=-646.511676$ a.u.

Free Energy after quasiharmonic app. $=-646.509540$ a.u.

C $\quad \begin{array}{llll}1.506193 & 0.193357 & -0.481346\end{array}$

C $\quad 2.140559 \quad 1.091970 \quad 0.609579$

H $\quad \begin{array}{llll}\text { H.935376 } & 0.659830 & 1.595487\end{array}$

H $\quad \begin{array}{llll}1.673422 & 2.080112 & 0.599344\end{array}$

C $2.218636-1.172380-0.526313$
H $\quad \begin{array}{lll}1.806937 & -1.783278 & -1.334326\end{array}$

H $\quad 2.014885-1.714615 \quad 0.402831$

C $3.733531-1.029911-0.703199$

H $\quad 4.206691-2.015544-0.687742$

H $3.941245-0.599453 \quad-1.689221$

$\begin{array}{llll}\text { C } & 3.655726 & 1.230850 & 0.430807\end{array}$

H $3.857268 \quad 1.775242 \quad-0.498294$

H $4.073162 \quad 1.832831 \quad 1.242615$

$\begin{array}{llll}\text { C } & 4.347789 & -0.133589 & 0.375427\end{array}$

H $5.418799-0.0064120 .197085$

H $\quad 4.249717 \quad-0.625595 \quad 1.349590$

H $\quad 1.674422 \quad 0.694270-1.443367$

B $\quad-0.049265 \quad 0.082419-0.271304$

$\begin{array}{llll}\text { O } & -0.662249 & -0.905371 & 0.459420\end{array}$

$\begin{array}{llll}0 & -0.947926 & 0.990165 & -0.777933\end{array}$

$\begin{array}{llll}\text { C } & -2.065526 & -0.539462 & 0.639847\end{array}$

$\begin{array}{llll}\text { C } & -2.297496 & 0.484747 & -0.536471\end{array}$

$\begin{array}{llll}\text { C } & -2.179201 & 0.089625 & 2.027740\end{array}$

H $\quad-3.212311 \quad 0.341688 \quad 2.267459$

H $\quad-1.823837 \quad-0.625676 \quad 2.770114$

$\begin{array}{llll}\text { H } & -1.574812 & 0.992961 & 2.111034\end{array}$

$\begin{array}{llll}\text { C } & -2.907070 & -1.804546 & 0.570667\end{array}$

H $\quad-2.658197 \quad-2.454148 \quad 1.410811$

H $\quad-3.969613 \quad-1.565545 \quad 0.635500$

H $\quad-2.732171-2.358891 \quad-0.349123$

C $\quad-3.202290 \quad 1.660149-0.200000$

H $\quad-3.275795 \quad 2.322154-1.063773$

$\begin{array}{llll}H & -4.208238 & 1.315460 & 0.044244\end{array}$

$\begin{array}{llll}\mathrm{H} & -2.821308 & 2.238878 & 0.638894\end{array}$

C $\quad-2.756711-0.173177 \quad-1.836993$

H $\quad-3.781796-0.535889-1.760154$

H $\quad-2.717168 \quad 0.564323 \quad-2.639240$

H $\quad-2.113801-1.008955-2.113618$

1Bpin $_{\text {eq }} 2^{\text {nd }}$ (B3LYP/def2-TZVPP, $\left.183 \mathrm{~K}, \mathrm{DCM}\right)$ :

Imaginary frequencies- 0

Electronic Energy (SCF) $=-646.828809059$ a.u

ZPV corrected Energy $=-646.489206$ a.u.

Enthalpy $=-646.482219$ a.u.

Free Energy = -646.511527 a.u.

Free Energy after quasiharmonic app. $=-646.509666$ a.u.

C $\quad-1.508441 \quad 0.117029-0.485143$

C $-2.227504-1.237636-0.340109$

H $\quad-2.026673 \quad-1.6459910 .655830$

H $\quad-1.818616-1.957350 \quad-1.054527$

C $\quad-2.136813 \quad 1.161518 \quad 0.470853$

H $\quad-1.663724 \quad 2.135966 \quad 0.324724$

H $\quad-1.933996 \quad 0.868370 \quad 1.507080$

C $\quad-3.651152 \quad 1.282896 \quad 0.273443$

H $\quad-4.065332 \quad 1.993744 \quad 0.993750$

H $\quad-3.849245 \quad 1.694608$-0.722288

C $-3.741645-1.113247-0.536320$

H $\quad-3.946883-0.823018-1.572842$

H $\quad-4.220485-2.084457 \quad-0.384413$

C $-4.351120 \quad-0.072173 \quad 0.406591$

H $\quad-5.4212420 .0353010 .210732$

H $\quad-4.256909-0.424934 \quad 1.439733$

$\begin{array}{llll}\text { H } & -1.675943 & 0.481731 & -1.507141\end{array}$

B $\quad 0.048184 \quad 0.035798 \quad-0.272442$

$\begin{array}{llll}\text { O } & 0.670041 & -0.819358 & 0.603329\end{array}$

$\begin{array}{lllll}\text { O } & 0.940553 & 0.847724 & -0.930624\end{array}$ 


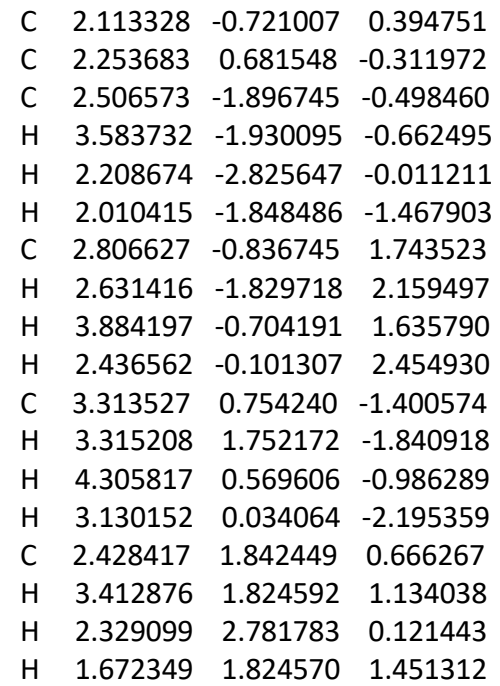

1 Bpin $_{\text {eq }} 3^{\text {rd }}$ (B3LYP/def2-TZVPP, $\left.183 \mathrm{~K}, \mathrm{DCM}\right):$ Imaginary frequencies- 0

Electronic Energy (SCF) $=-646.828749473$ a.u ZPV corrected Energy = -646.489281 a.u. Enthalpy $=-646.482312$ a.u.

Free Energy = -646.512292 a.u.

Free Energy after quasiharmonic app. $=-646.509747$ a.u.

C $-1.509545-0.073899-0.417327$

C $\quad-2.193360 \quad-1.226829 \quad 0.346265$

H $\quad-2.030365-1.091217 \quad 1.421697$

$\begin{array}{llll}\text { H } & -1.727661 & -2.178758 & 0.082912\end{array}$

$\begin{array}{llll}\text { C } & -2.211814 & 1.265668 & -0.123087\end{array}$

H $\quad-1.759762 \quad 2.064158-0.715398$

H $\quad-2.048695 \quad 1.533036 \quad 0.927176$

C $\quad-3.718130 \quad 1.200728-0.393687$

H $\quad-4.188541 \quad 2.151378-0.127851$

H $\quad-3.882855 \quad 1.060743 \quad-1.468039$

C $\quad-3.699533 \quad-1.286186 \quad 0.074269$

H $\quad-3.862634-1.548579-0.977118$

$\begin{array}{llll}\text { H } & -4.156810 & -2.081947 & 0.668719\end{array}$

$\begin{array}{llll}\text { C } & -4.383181 & 0.051757 & 0.369185\end{array}$

H $\quad-5.445984-0.003350 \quad 0.119045$

H $\quad-4.326326 \quad 0.254483 \quad 1.444641$

H $\quad-1.644706 \quad-0.280366 \quad-1.491454$

B $\quad 0.049613 \quad-0.031504 \quad-0.202844$

$\begin{array}{lllll}\text { O } & 0.801623 & 1.117064 & -0.243419\end{array}$

$\begin{array}{llll}\text { O } & 0.819062 & -1.149792 & 0.011264\end{array}$

C 2.1666320 .7872620 .159893

C $2.224304 \quad-0.766420-0.099482$

C $\quad 2.290335 \quad 1.164340 \quad 1.635598$

H $3.302600 \quad 1.0000802 .005306$

H $\quad 2.055045 \quad 2.222838 \quad 1.750895$

$\begin{array}{llll}\text { H } & 1.598677 & 0.594167 & 2.255935\end{array}$

$\begin{array}{llll}\text { C } & 3.131558 & 1.617873 & -0.672235\end{array}$

H $2.996972 \quad 2.674946 \quad-0.439333$

H $4.164992 \quad 1.353484-0.443251$

H $\quad 2.967811 \quad 1.483094 \quad-1.739422$

C $3.025608-1.562375 \quad 0.919217$

H $\quad 2.985877 \quad-2.622698 \quad 0.666353$

H $4.072486-1.255297 \quad 0.911577$

H $\quad 2.636129-1.4403801 .927837$
C $2.668433-1.131869-1.515361$

H $3.728139-0.925537-1.666405$

H $2.504718-2.198378-1.672976$

H $2.099503 \quad-0.586857 \quad-2.268862$

Substituted cyclohexylacetylenes (4R):

$4 \mathrm{Me}_{\mathrm{ax}}$

Imaginary frequencies- 0

Electronic Energy (SCF) $=-349.037614123$ a.u

Electronic Energy (MP2) $=-350.5712801455$ a.u

ZPV corrected Energy = -350.362275 a.u.

Enthalpy $=-350.351659$ a.u.

Free Energy $=-350.398506$ a.u.

C $\quad-1.206592-0.143302-0.030688$

C $-0.842783-0.9274301 .241100$

H $\quad-1.423892 \quad-1.855148 \quad 1.246069$

H $\quad-1.133237 \quad-0.351998 \quad 2.121766$

C $-0.783389-0.943747-1.273660$

H $\quad-1.031607-0.379853-2.174498$

H $-1.364329-1.871357-1.294071$

C $0.704617-1.276488-1.237135$

H $\quad 0.977630 \quad-1.857622-2.120264$

H $\quad 1.277717-0.345696-1.273262$

C $0.645341-1.259421 \quad 1.279014$

H $\quad 1.215833 \quad-0.327614 \quad 1.329153$

H $\quad 0.876846 \quad-1.828125 \quad 2.181868$

C $1.061025 \quad-2.041946 \quad 0.035286$

H $\quad 2.131622-2.2547140 .061899$

H $\quad 0.544287 \quad-3.007686 \quad 0.029755$

H $\quad-2.291024-0.003273-0.057263$

C $\quad-0.575478 \quad 1.176128-0.024005$

$\begin{array}{llll}\text { C } & -0.018364 & 2.261186 & -0.016578\end{array}$

$\begin{array}{llll}\text { C } & 0.638378 & 3.564764 & -0.007908\end{array}$

H $\quad 0.013003 \quad 4.3111090 .480838$

H $\quad 1.587154 \quad 3.517743 \quad 0.525936$

H $\quad 0.838883 \quad 3.904940 \quad-1.023351$

$4 \mathrm{Me}_{\text {eq }}$

Imaginary frequencies- 0

Electronic Energy (SCF) $=-349.040177104$ a.u Electronic Energy (MP2) $=-350.5708185867$ a.u ZPV corrected Energy $=-350.361935$ a.u. Enthalpy $=-350.351247$ a.u.

Free Energy $=-350.397947$ a.u.

C $\quad 0.494145 \quad 0.014718 \quad 0.000000$

$\begin{array}{llll}\text { C } & -0.187835 & -0.538526 & 1.258771\end{array}$

H $\quad-1.216875 \quad-0.166580 \quad 1.282136$

H $\quad 0.314713 \quad-0.149573 \quad 2.146136$

C $-0.187835-0.538526 \quad-1.258771$

H $\quad 0.314713-0.149573-2.146136$

H $\quad-1.216875-0.166580-1.282136$

C $-0.187835-2.064109-1.256202$

H $\quad-0.688776-2.438728-2.151028$ 


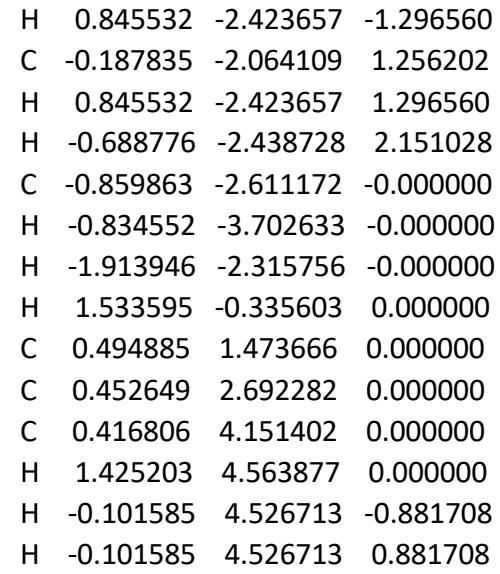

$4 \mathrm{tBu}_{\mathrm{ax}}$

Imaginary frequencies- 0

Electronic Energy (SCF) = -466.179898454 a.u Electronic Energy (MP2) = -468.2547612784 a.u ZPV corrected Energy = -467.960688 a.u.

Enthalpy $=-467.946293$ a.u.

Free Energy = -468.002312 a.u.

$\begin{array}{llll}\text { C } & 1.385000 & 0.009292 & -1.308792\end{array}$

C $2.111442-1.252956-0.813591$

H $\quad 3.114037-1.262815 \quad-1.253093$

H $1.586155 \quad-2.140866-1.169794$

C $2.114926 \quad 1.262987-0.797161$

H $\quad 1.592046 \quad 2.157094 \quad-1.141232$

H $3.117293 \quad 1.275852 \quad-1.237050$

C $2.226417 \quad 1.253866 \quad 0.724041$

H $2.760667 \quad 2.143611 \quad 1.063358$

H $\quad 1.221214 \quad 1.297127 \quad 1.153354$

C $2.221869-1.2640950 .707684$

H $\quad 1.216313-1.309440 \quad 1.136075$

H $2.752888-2.159957 \quad 1.035802$

C $\quad 2.932217-0.009603 \quad 1.211738$

H $2.984910 \quad-0.016780 \quad 2.302335$

H $3.963622 \quad-0.009095 \quad 0.842937$

H $\quad 1.397561 \quad 0.016425 \quad-2.402451$

C $\quad-0.006557 \quad 0.007840 \quad-0.861040$

C $\quad-1.149340 \quad 0.003601 \quad-0.426757$

C $-2.512685 \quad-0.000518 \quad 0.107340$

C $-2.508736-0.672414 \quad 1.485255$

H $\quad-3.519665 \quad-0.679800 \quad 1.897870$

H $\quad-2.155341-1.700961 \quad 1.409175$

H $\quad-1.855597 \quad-0.133768 \quad 2.172386$

C $\quad-3.010423 \quad 1.442854 \quad 0.241918$

H $\quad-3.016581 \quad 1.938216 \quad-0.728999$

H $\quad-4.025440 \quad 1.4488290 .644287$

H $\quad-2.365348 \quad 2.009713 \quad 0.913494$

C $\quad-3.433736-0.776068-0.840456$

H $\quad-3.093883 \quad-1.806206 \quad-0.947593$

H $\quad-4.451334 \quad-0.782738-0.444740$

H $\quad-3.445880 \quad-0.313154-1.827195$
$4 \mathrm{tBu}$ eq

Imaginary frequencies- 0

Electronic Energy (SCF) = -466.182879073 a.u Electronic Energy (MP2) $=-468.2537853803$ a.u ZPV corrected Energy = -467.959871 a.u.

Enthalpy $=-467.945357$ a.u.

Free Energy $=-468.002201$ a.u.

C $\quad-0.362881 \quad 1.231671 \quad 0.000000$

C $0.336072 \quad 1.763681 \quad 1.258979$

H $\quad \begin{array}{llll}1.353076 & 1.359758 & 1.281648\end{array}$

$\begin{array}{llll}H & -0.178070 & 1.389937 & 2.146311\end{array}$

C $0.336072 \quad 1.763681 \quad-1.258979$

H $\quad-0.178070 \quad 1.389937 \quad-2.146311$

H $\quad 1.353076 \quad 1.359758$ - 1.281648

C $0.383312 \quad 3.288486-1.256330$

H $\quad 0.895703 \quad 3.647337 \quad-2.151142$

H $\quad-0.638400 \quad 3.679899 \quad-1.296727$

C $0.383312 \quad 3.288486 \quad 1.256330$

$\begin{array}{llll}\text { H } & -0.638400 & 3.679899 & 1.296727\end{array}$

H $\quad 0.895703 \quad 3.647337 \quad 2.151142$

C $\quad 1.072000 \quad 3.814316 \quad 0.000000$

H $\quad 1.080764 \quad 4.906033 \quad 0.000000$

H $\quad 2.116359 \quad 3.486164 \quad 0.000000$

H $\quad-1.391782 \quad 1.6121680 .000000$

$\begin{array}{llll}\text { C } & -0.405080 & -0.226188 & -0.000000\end{array}$

C $-0.392560-1.448403-0.000000$

C $-0.388556-2.912754-0.000000$

C $-1.832615-3.426158-0.000000$

H $\quad-2.364411 \quad-3.074604 \quad-0.884175$

H $\quad-1.837205-4.518010-0.000000$

H $\quad-2.364411-3.074604 \quad 0.884175$

C $0.336072-3.419171-1.252032$

H $\quad 1.365941 \quad-3.062388$ - 1.269486

H $\quad 0.343888-4.510991-1.259203$

H $\quad-0.165452 \quad-3.067547 \quad-2.153707$

$\begin{array}{llll}\text { C } & 0.336072 & -3.419171 & 1.252032\end{array}$

H $\quad 0.343888-4.510991 \quad 1.259203$

H $\quad 1.365941 \quad-3.062388 \quad 1.269486$

$\begin{array}{llll}\text { H } & -0.165452 & -3.067547 & 2.153707\end{array}$

\footnotetext{
4SiMe $3 a x$

Imaginary frequencies- 0

Electronic Energy (SCF) = -717.296650330 a.u ZPV corrected Energy = -719.036059 a.u.

Enthalpy $=-719.019467$ a.u.

Free Energy $=-719.081707$ a.u.

$\begin{array}{llll}\text { C } & 1.798416 & 0.005145 & -1.323058\end{array}$

C $2.505550-1.256892-0.798703$

H $3.521436-1.268291-1.206022$

H $\quad \begin{array}{llll}1.991448 & -2.145531 & -1.168798\end{array}$

C $2.508787 \quad 1.261855 \quad-0.790358$

H $\quad \begin{array}{llll}\text { H } & 1.996818 & 2.154337 & -1.154101\end{array}$

H $\quad 3.524495 \quad 1.273440 \quad-1.198081$

$\begin{array}{llll}\text { C } & 2.570094 & 1.256141 & 0.733637\end{array}$
} Electronic Energy (MP2) $=-719.3194929038$ a.u 


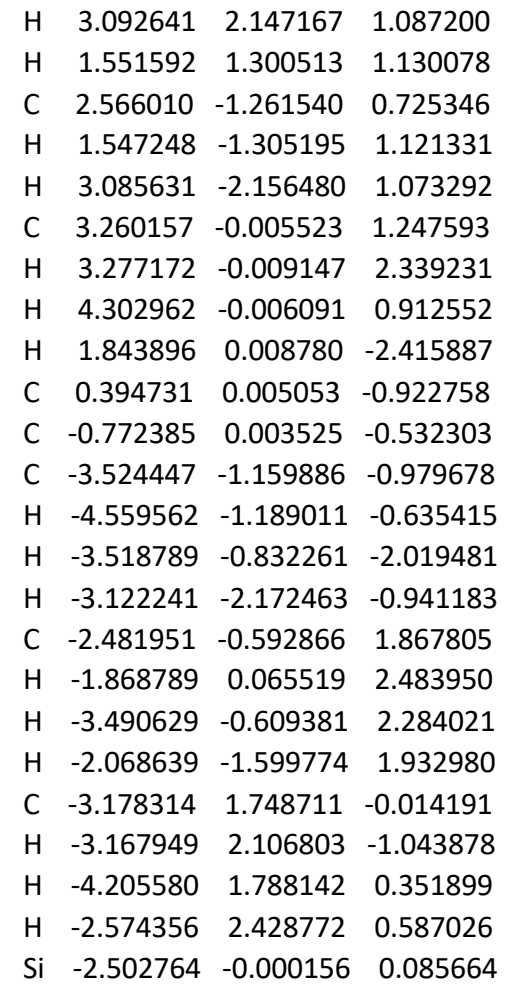

\section{SiMe}

Imaginary frequencies- 0

Electronic Energy (SCF) = -717.299468316 a.u Electronic Energy (MP2) = -719.3185256549 a.u ZPV corrected Energy = -719.035248 a.u. Enthalpy $=-719.018544$ a.u.

Free Energy = -719.081170 a.u.

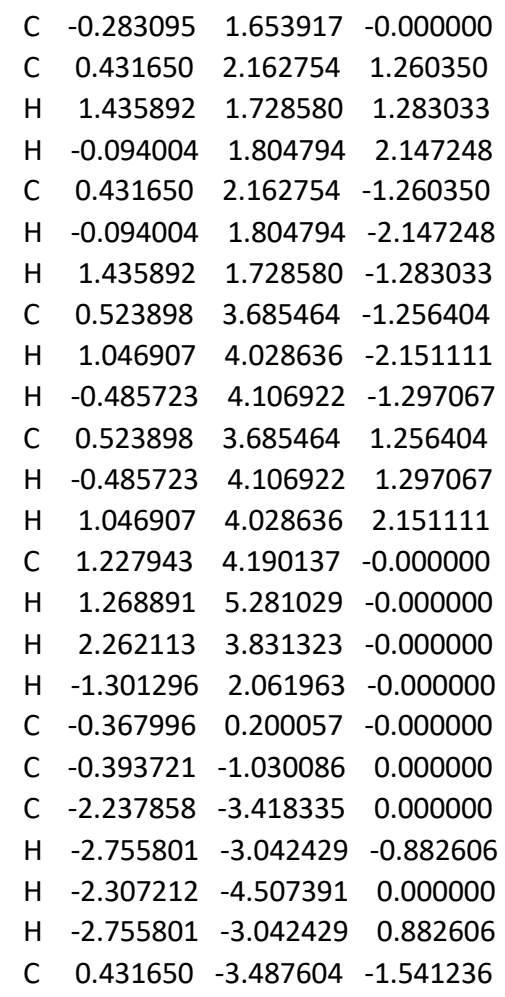

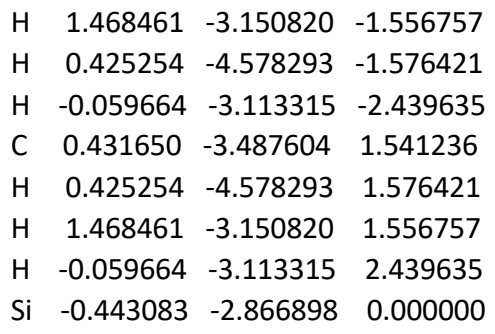


Electronic Energy (SCF) = -543.112079922 a.u Electronic Energy (MP2) $=-545.5129174833$ a.u ZPV corrected Energy = -545.179888 a.u. Enthalpy $=-545.165279$ a.u.

Free Energy $=-545.222683$ a.u.

C $-2.387658-0.836050-0.931240$

C $-3.0773340 .509752-1.213089$

H $\quad-4.152577 \quad 0.327527 \quad-1.308736$

H $\quad-2.724226 \quad 0.909404-2.165310$

$\begin{array}{llll}\text { C } & -2.873675 & -1.395896 & 0.416613\end{array}$

H $\quad-2.376144 \quad-2.345975 \quad 0.619176$

H $\quad-3.947046 \quad-1.595066 \quad 0.335000$

$\begin{array}{llll}\text { C } & -2.624476 & -0.403456 & 1.547830\end{array}$

$\begin{array}{llll}\text { H } & -2.990584 & -0.814425 & 2.490877\end{array}$

H $\quad-1.546328 \quad-0.254128 \quad 1.657199$

$\begin{array}{llll}\text { C } & -2.827510 & 1.504067 & -0.083674\end{array}$

H $\quad-1.756496 \quad 1.721038-0.032701$

H $\quad-3.337549 \quad 2.445658 \quad-0.297020$

C $\quad-3.295141 \quad 0.937060 \quad 1.255104$

H $\quad-3.089798 \quad 1.646674 \quad 2.059208$

H $\quad-4.380916 \quad 0.795502 \quad 1.224757$

H $\quad-2.655582-1.541638-1.722866$

$\begin{array}{llll}\text { C } & -0.934891 & -0.675119 & -0.924453\end{array}$

$\begin{array}{llll}\text { C } & 0.273735 & -0.498770 & -0.880293\end{array}$

C $1.708484-0.267897 \quad-0.759709$

C $\quad \begin{array}{llll}1.990500 & 1.167634 & -0.293703\end{array}$

H $\quad 1.541758 \quad 1.873062-0.995657$

H $\quad 1.496733 \quad 1.315890 \quad 0.672184$

C $2.349654-1.2737420 .206563$

H $\quad 1.862870-1.1686801 .181405$

H $\quad 2.154068-2.289254-0.142409$

H $\quad 2.173874-0.401543 \quad-1.743990$

C $3.489322 \quad 1.414794-0.152940$

H $3.668435 \quad 2.4347320 .192995$

H $3.962114 \quad 1.325096-1.136364$

C $3.847874-1.0218290 .345288$

H $4.331834-1.189942 \quad-0.622312$

H $\quad 4.283169-1.737199 \quad 1.045831$

C 4.1240560 .4078810 .802205

H $\quad \begin{array}{llll}5.199205 & 0.581722 & 0.878097\end{array}$

H $3.705544 \quad 0.550966 \quad 1.803616$
4 Cyeqeq $_{\text {eq }}$

Imaginary frequencies- 0

Electronic Energy (SCF) $=-543.115157515$ a.u Electronic Energy (MP2) $=-545.5118468906$ a.u ZPV corrected Energy = -545.178986 a.u. Enthalpy $=-545.164248$ a.u.

Free Energy $=-545.222456$ a.u.

C $2.034880 \quad-0.374763 \quad 0.373680$

C $\quad 2.524043 \quad 1.058883 \quad 0.622694$

H $\quad 1.975983 \quad 1.489705 \quad 1.462603$

H $\quad 2.284795 \quad 1.659633 \quad-0.260370$

C $2.809454-1.003779-0.792816$

H $\quad 2.575641 \quad-0.440216 \quad-1.701442$

H $\quad 2.462624-2.026563-0.950712$

C $4.312531-0.970223-0.533713$

H $4.846686-1.408541-1.379052$

H $4.540506 \quad-1.587998 \quad 0.341008$

$\begin{array}{llll}\text { C } 4.027750 & 1.088260 & 0.878575\end{array}$

H $\quad 4.246731 \quad 0.536610 \quad 1.798628$

H $\quad 4.358887 \quad 2.116190 \quad 1.039057$

$\begin{array}{llll}\text { C } & 4.794203 & 0.455403 & -0.279485\end{array}$

H $4.636309 \quad 1.053290 \quad-1.182796$

H $\quad \begin{array}{llll}5.866631 & 0.463532 & -0.075044\end{array}$

H $\quad 2.246596 \quad-0.963629 \quad 1.274734$

$\begin{array}{llll}\text { C } & 0.597911 & -0.402314 & 0.125745\end{array}$

$\begin{array}{llll}\text { C } & -0.597919 & -0.402356 & -0.126104\end{array}$

$\begin{array}{llll}\text { C } & -2.034917 & -0.374835 & -0.373849\end{array}$

C $-2.809310-1.0037310 .792830$

H $\quad-2.462475-2.0265090 .950751$

H $\quad-2.575331-0.440101 \quad 1.701371$

C $\quad-2.524137 \quad 1.058783 \quad-0.622916$

$\begin{array}{llll}\text { H } & -2.284723 & 1.659607 & 0.260049\end{array}$

H $\quad-1.976248 \quad 1.489546 \quad-1.462970$

H $\quad-2.246786-0.963796 \quad-1.274806$

$\begin{array}{llll}\text { C } & -4.312438 & -0.970185 & 0.534014\end{array}$

H $\quad-4.846407 \quad-1.408414 \quad 1.379520$

H $\quad-4.540607 \quad-1.588060-0.340584$

C $\quad-4.027894 \quad 1.088156 \quad-0.878524$

H $\quad-4.247065 \quad 0.536440 \quad-1.798492$

$\begin{array}{lllll}H & -4.359032 & 2.116081 & -1.039034\end{array}$

$\begin{array}{lllll}\text { C } & -4.794155 & 0.455415 & 0.279728\end{array}$

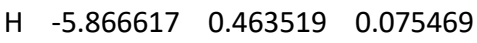

H $\quad-4.636109 \quad 1.053391 \quad 1.182955$ 


\section{Bibliography}

(1) Møller, Chr.; Plesset, M. S. Note on an Approximation Treatment for Many-Electron Systems. Phys. Rev. 1934, 46 (7), 618-622. https://doi.org/10.1103/PhysRev.46.618.

(2) Pritchard, B. P.; Altarawy, D.; Didier, B.; Gibson, T. D.; Windus, T. L. New Basis Set Exchange: An Open, Up-to-Date Resource for the Molecular Sciences Community. J. Chem. Inf. Model. 2019, 59 (11), 48144820. https://doi.org/10.1021/acs.jcim.9b00725.

(3) Becke, A. D. Density-functional Thermochemistry. III. The Role of Exact Exchange. J. Chem. Phys. 1993, 98 (7), 5648-5652. https://doi.org/10.1063/1.464913.

(4) Lee, C.; Yang, W.; Parr, R. G. Development of the Colle-Salvetti Correlation-Energy Formula into a Functional of the Electron Density. Phys. Rev. B 1988, 37 (2), 785-789. https://doi.org/10.1103/PhysRevB.37.785.

(5) Weigend, F.; Ahlrichs, R. Balanced Basis Sets of Split Valence, Triple Zeta Valence and Quadruple Zeta Valence Quality for H to Rn: Design and Assessment of Accuracy. Phys. Chem. Chem. Phys. 2005, 7 (18), 3297-3305. https://doi.org/10.1039/b508541a.

(6) Grimme, S.; Antony, J.; Ehrlich, S.; Krieg, H. A Consistent and Accurate Ab Initio Parametrization of Density Functional Dispersion Correction (DFT-D) for the 94 Elements H-Pu. J. Chem. Phys. 2010, 132 (15), 154104. https://doi.org/10.1063/1.3382344.

(7) Grimme, S.; Ehrlich, S.; Goerigk, L. Effect of the Damping Function in Dispersion Corrected Density Functional Theory. J. Comput. Chem. 2011, 32 (7), 1456-1465. https://doi.org/10.1002/jcc.21759.

(8) Frisch, M. J.; Trucks, G. W.; Schlegel, H. B.; Scuseria, G. E.; Robb, M. A.; Cheeseman, J. R.; Scalmani, G.; Barone, V.; Petersson, G. A.; Nakatsuji, H.; Li, X.; Caricato, M.; Marenich, A. V.; Bloino, J.; Janesko, B. G.; Gomperts, R.; Mennucci, B.; Hratchian, H. P.; Ortiz, J. V.; Izmaylov, A. F.; Sonnenberg, J. L.; WilliamsYoung, D.; Ding, F.; Lipparini, F.; Egidi, F.; Goings, J.; Peng, B.; Petrone, A.; Henderson, T.; Ranasinghe, D.; Zakrzewski, V. G.; Gao, J.; Rega, N.; Zheng, G.; Liang, W.; Hada, M.; Ehara, M.; Toyota, K.; Fukuda, R.; Hasegawa, J.; Ishida, M.; Nakajima, T.; Honda, Y.; Kitao, O.; Nakai, H.; Vreven, T.; Throssell, K.; Montgomery, Jr., J. A.; Peralta, J. E.; Ogliaro, F.; Bearpark, M. J.; Heyd, J. J.; Brothers, E. N.; Kudin, K. N.; Staroverov, V. N.; Keith, T. A.; Kobayashi, R.; Normand, J.; Raghavachari, K.; Rendell, A. P.; Burant, J. C.; Iyengar, S. S.; Tomasi, J.; Cossi, M.; Millam, J. M.; Klene, M.; Adamo, C.; Cammi, R.; Ochterski, J. W.; Martin, R. L.; Morokuma, K.; Farkas, O.; Foresman, J. B.; Fox, D. J. Gaussian 16 (Revision C.01); Gaussian, Inc., Wallingford CT, 2016.

(9) Riplinger, C.; Sandhoefer, B.; Hansen, A.; Neese, F. Natural Triple Excitations in Local Coupled Cluster Calculations with Pair Natural Orbitals. J. Chem. Phys. 2013, 139 (13), 134101.

https://doi.org/10.1063/1.4821834.

(10) Neese, F. The ORCA Program System. WIREs Comput. Mol. Sci. 2012, 2 (1), 73-78. https://doi.org/10.1002/wcms.81.

(11) Caldeweyher, E.; Bannwarth, C.; Grimme, S. Extension of the D3 Dispersion Coefficient Model. J. Chem. Phys. 2017, 147 (3), 034112. https://doi.org/10.1063/1.4993215.

(12) Caldeweyher, E.; Ehlert, S.; Hansen, A.; Neugebauer, H.; Spicher, S.; Bannwarth, C.; Grimme, S. A Generally Applicable Atomic-Charge Dependent London Dispersion Correction. J. Chem. Phys. 2019, 150 (15), 154122. https://doi.org/10.1063/1.5090222.

(13) Contreras-García, J.; Johnson, E. R.; Keinan, S.; Chaudret, R.; Piquemal, J.-P.; Beratan, D. N.; Yang, W. NCIPLOT: A Program for Plotting Noncovalent Interaction Regions. J. Chem. Theory Comput. 2011, 7 (3), 625-632. https://doi.org/10.1021/ct100641a.

(14) Lu, T.; Chen, F. Multiwfn: A Multifunctional Wavefunction Analyzer. J. Comput. Chem. 2012, 33 (5), $580-$ 592. https://doi.org/10.1002/jcc.22885.

(15) Humphrey, W.; Dalke, A.; Schulten, K. VMD: Visual Molecular Dynamics. J. Mol. Graph. 1996, 14 (1), $33-$ 38. https://doi.org/10.1016/0263-7855(96)00018-5.

(16) Dommen, J.; Brupbacher, T.; Grassi, G.; Bauder, A. Microwave Spectra of Isotopic Species and Substitution Structure of Cyclohexane. J. Am. Chem. Soc. 1990, 112 (3), 953-957. https://doi.org/10.1021/ja00159a007.

(17) Białkowska-Jaworska, E.; Jaworski, M.; Kisiel, Z. The Structure of Cyclohexane, F-, Cl-, Br- and ICyclohexane. J. Mol. Struct. 1995, 350 (3), 247-254. https://doi.org/10.1016/0022-2860(94)08481-V.

(18) Durig, J. R.; Zheng, C.; El Defrawy, A. M.; Ward, R. M.; Gounev, T. K.; Ravindranath, K.; Rajeswara Rao, N. On the Relative Intensities of the Raman Active Fundamentals, $r_{0}$ Structural Parameters, and Pathway of Chair-Boat Interconversion of Cyclohexane and Cyclohexane- $d_{12}$. J. Raman Spectrosc. 2009, 40 (2), 197204. https://doi.org/10.1002/jrs.2107. 
(19) Riehn, C.; Matylitsky, V. V.; Jarzęba, W.; Brutschy, B.; Tarakeshwar, P.; Kim, K. S. Insights into the Structure of Cyclohexane from Femtosecond Degenerate Four-Wave Mixing Spectroscopy and Ab Initio Calculations. J. Am. Chem. Soc. 2003, 125 (52), 16455-16462. https://doi.org/10.1021/ja036615o.

(20) Bak, K. L.; Gauss, J.; Jørgensen, P.; Olsen, J.; Helgaker, T.; Stanton, J. F. The Accurate Determination of Molecular Equilibrium Structures. J. Chem. Phys. 2001, 114 (15), 6548-6556. https://doi.org/10.1063/1.1357225.

(21) Jeziorski, B.; Moszynski, R.; Szalewicz, K. Perturbation Theory Approach to Intermolecular Potential Energy Surfaces of van Der Waals Complexes. Chem. Rev. 1994, 94 (7), 1887-1930. https://doi.org/10.1021/cr00031a008.

(22) Hohenstein, E. G.; Sherrill, C. D. Density Fitting of Intramonomer Correlation Effects in SymmetryAdapted Perturbation Theory. J. Chem. Phys. 2010, 133 (1), 014101. https://doi.org/10.1063/1.3451077.

(23) Žabka, M.; Naviri, L.; Gschwind, R. M. Noncovalent $\mathrm{CH}-\pi$ and $\pi-\pi$ Interactions in Phosphoramidite Palladium(II) Complexes with Strong Conformational Preference. Angew. Chem. Int. Ed. 2021, Accepted. https://doi.org/10.1002/anie.202106881.

(24) Ho, L. P.; Nasr, A.; Jones, P. G.; Altun, A.; Neese, F.; Bistoni, G.; Tamm, M. London Dispersion Interactions in Pnictogen Cations $\left[\mathrm{ECl}_{2}\right]^{+}$and $[\mathrm{E}=\mathrm{E}]^{2+}(\mathrm{E}=\mathrm{P}, \mathrm{As}, \mathrm{Sb})$ Supported by Anionic $N$-Heterocyclic Carbenes. Chem. - Eur. J. 2018, 24, 18922-18932. https://doi.org/10.1002/chem.201804714.

(25) Lu, Q.; Neese, F.; Bistoni, G. Formation of Agostic Structures Driven by London Dispersion. Angew. Chem. Int. Ed. 2018, 57 (17), 4760-4764. https://doi.org/10.1002/anie.201801531.

(26) Fritzsche, A.-M.; Scholz, S.; Krasowska, M.; Bhattacharyya, K.; Toma, A. M.; Silvestru, C.; Korb, M.; Rüffer, T.; Lang, H.; Auer, A. A.; Mehring, M. Evaluation of Bismuth-Based Dispersion Energy Donors - Synthesis, Structure and Theoretical Study of 2-Biphenylbismuth( III ) Derivatives. Phys. Chem. Chem. Phys. 2020, 22 (18), 10189-10211. https://doi.org/10.1039/C9CP06924K.

(27) Verma, P.; Wang, B.; Fernandez, L. E.; Truhlar, D. G. Physical Molecular Mechanics Method for Damped Dispersion. J. Phys. Chem. A 2017, 121 (14), 2855-2862. https://doi.org/10.1021/acs.jpca.7b02384.

(28) Bursch, M.; Caldeweyher, E.; Hansen, A.; Neugebauer, H.; Ehlert, S.; Grimme, S. Understanding and Quantifying London Dispersion Effects in Organometallic Complexes. Acc. Chem. Res. 2019, 52 (1), 258266. https://doi.org/10.1021/acs.accounts.8b00505.

(29) Lu, Q.; Neese, F.; Bistoni, G. London Dispersion Effects in the Coordination and Activation of Alkanes in б-Complexes: A Local Energy Decomposition Study. Phys. Chem. Chem. Phys. 2019, 21 (22), 1156911577. https://doi.org/10.1039/C9CP01309A.

(30) Eliel, E. L.; Wilen, S. H. In Stereochemistry of Organic Compounds; John Wiley \& Sons Inc: New York, 1994; pp 696-697.

(31) Brethomé, A. V.; Fletcher, S. P.; Paton, R. S. Conformational Effects on Physical-Organic Descriptors: The Case of Sterimol Steric Parameters. ACS Catal. 2019, 9 (3), 2313-2323. https://doi.org/10.1021/acscatal.8b04043.

(32) Bondi, A. Van Der Waals Volumes and Radii. J. Phys. Chem. 1964, 68 (3), 441-451. https://doi.org/10.1021/j100785a001. 